\title{
Auswirkungen der Umsetzung von Naturschutzmaß- nahmen auf die Forst- und Holzwirtschaft
}

\author{
Dissertation \\ zur Erlangung des Doktorgrades \\ der Fakultät für Forstwissenschaften und Waldökologie \\ der Georg-August-Universität Göttingen
}

vorgelegt von

Lydia Christina Antonia Rosenkranz

geboren in Bonn

Göttingen, 2015 
1. Gutachter: Prof. Dr. Matthias Dieter

2. Gutachter: Prof. Dr. Bernhard Möhring

Tag der mündlichen Prüfung: 31. März 2016 




\section{Inhaltsverzeichnis}

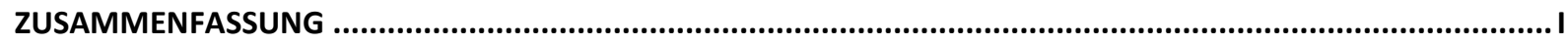

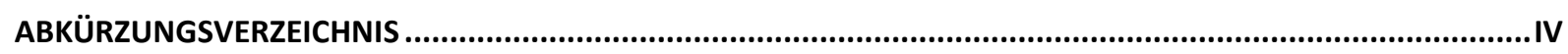

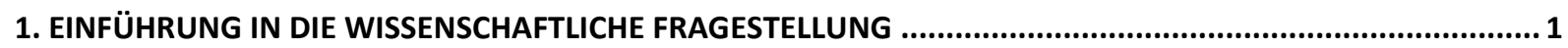

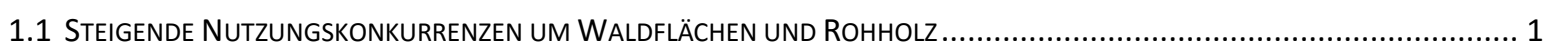

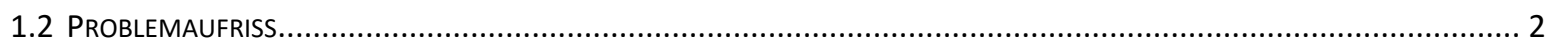

1.2.1 Zur Situation der Forstwirtschaft in Deutschland ............................................................... 2

1.2.2 Zur Situation der Holzwirtschaft in Deutschland ................................................................. 5

1.2.3 Zur Situation des Waldnaturschutzes in Deutschland .............................................................. 7

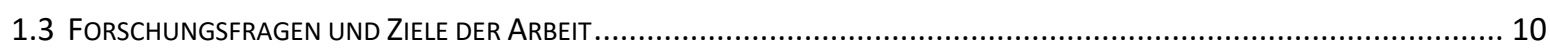

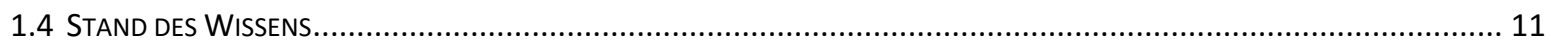

1.4.1 Herausforderungen bei der Bewertung der Auswirkungen naturschutzfachlicher

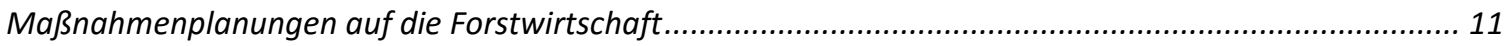

1.4.2 Bewertung der Auswirkungen naturschutzfachlicher Maßnahmen auf die Forstwirtschaft ............. 13

1.4.3 Herausforderungen bei der Bewertung von Preis- und Mengenänderungen auf die Holzwirtschaft. 20

1.4.4 Bewertung der Auswirkungen von verändertem Holzeinschlag auf die Holzwirtschaft.................... 21

2 EINORDNUNG DER EIGENEN ERGEBNISSE IN DEN FACHLICHEN KONTEXT ............................................25

2.1 ERSTER ARTIKEL: „INCOME LOSSES DUE TO THE IMPLEMENTATION OF THE HABITATS DIRECTIVE IN FORESTS - CONCLUSIONS

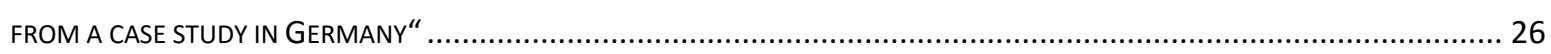

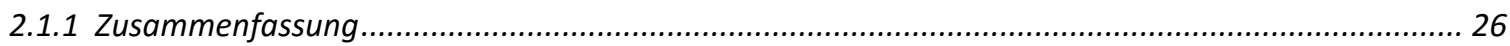

2.1.2 Kritische Diskussion und Einordnung in den thematischen Rahmen ............................................ 28

2.2 ZWEITER ARTIKEL: „OPPORTUNITÄTSKOSTENANALYSE ZUR IMPLEMENTIERUNG DES NATURSCHUTZORIENTIERTEN

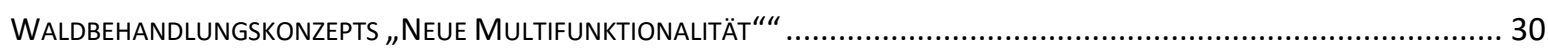

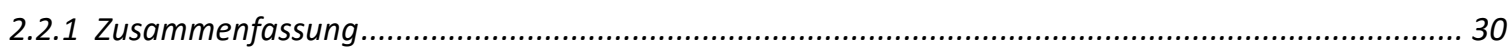

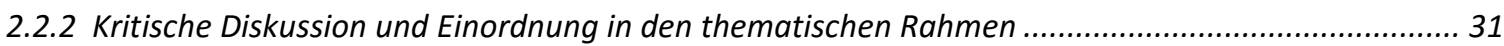

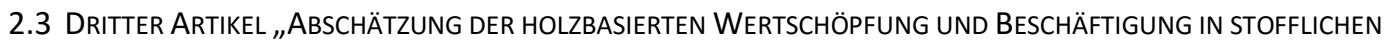

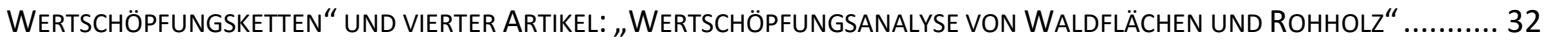

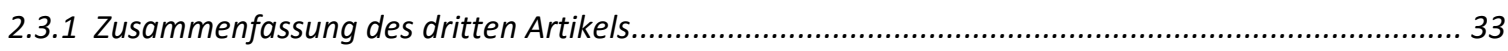

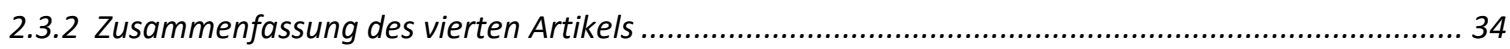

2.3.4 Kritische Diskussion und Einordnung in den thematischen Rahmen ......................................... 36

2.4 FÜNFTER ARTIKEL „DECOMPOSITION ANALYSIS OF CHANGES IN VALUE ADDED. A CASE STUDY OF THE SAWMILLING AND WOOD

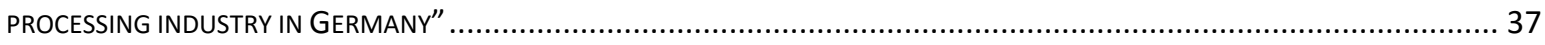

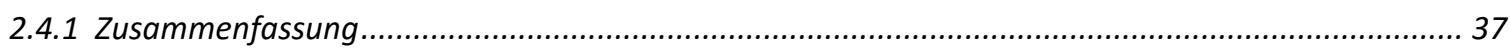

2.4.2 Kritische Diskussion und Einordnung in den thematischen Rahmen .......................................... 39

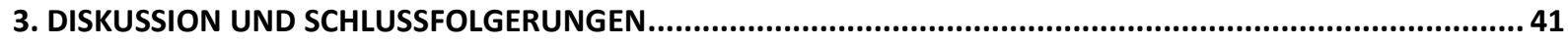

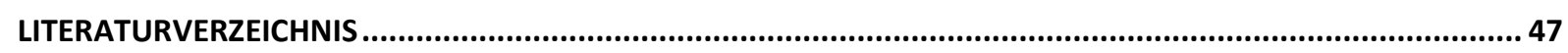

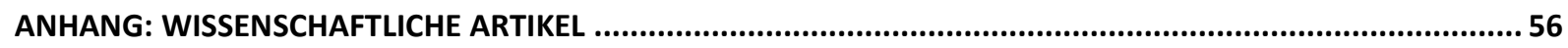

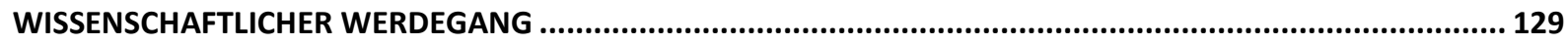

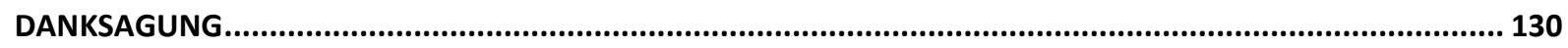

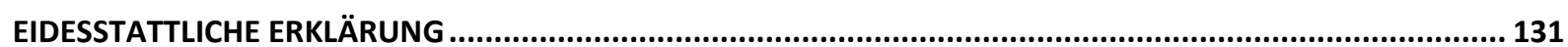





\section{Zusammenfassung}

In den letzten Jahren sind die gesellschaftlichen Ansprüche an die Waldnutzung deutlich gestiegen. Den begrenzten Produktionsmöglichkeiten des Waldes stehen vielfältige, teilweise konkurrierende Nutzungsansprüche gegenüber. Dies führt zu Nutzungskonkurrenzen um Waldflächen und Rohholz, die geregelt werden müssen. Die vielfach geforderte Balance aus ökologischem, ökonomischem und sozialem Nutzen kann unter Umständen im Zuge der multifunktionalen Waldwirtschaft zukünftig nicht mehr auf ganzer Fläche erbracht werden. Unter den vielen verschiedenen gesellschaftlichen Nutzungskonflikten waren und sind insbesondere die Nutzungskonkurrenzen zwischen Holznutzung (Forst- und Holzwirtschaft) und Nutzungsverzicht (Naturschutz) in den letzten Jahren von zentraler Bedeutung.

Für eine umfassende Analyse der Folgen der Umsetzung gesellschaftlicher Forderungen nach Waldund Holznutzung sind u.a. wissenschaftliche Bewertungsansätze erforderlich. Sie können einen wichtigen Beitrag für Politikfolgeabschätzungen und Effizienzbewertungen bei der Umsetzung konkurrierender Ansprüche an die Wald- und Holznutzung leisten.

Vor diesem Hintergrund ist das Ziel dieser Arbeit die Entwicklung und Anwendung von Methoden zur Bewertung der naturalen und ökonomischen Auswirkungen von Nutzungskonkurrenzen zwischen der Forst- und Holzwirtschaft und dem Naturschutz. Hierfür werden die folgenden wissenschaftlichen Fragestellungen bearbeitet: (1) „Welche Auswirkungen haben aktuelle naturschutzfachliche Maßnahmenplanungen auf die Forstwirtschaft?“ und (2) „Welche Auswirkungen hat der veränderte inländische Holzeinschlag auf die Holzindustrie?" Die Methoden und Ergebnisse der vorliegenden wissenschaftlichen Arbeit zur Bewertung von Nutzungskonkurrenzen wurden in drei peer-reviewten und zwei weiteren Artikeln in forst- und holzwirtschaftlichen Fachzeitschriften veröffentlicht.

Im letzten Jahrzehnt wurde zur Erhöhung des Waldnaturschutzniveaus die Schutzgebietskulisse durch die Ausweisung von FFH-Gebieten deutlich ausgeweitet. Die betroffenen, für den Naturschutz relevanten Waldflächen sind jedoch ebenso wichtig für den Betriebserfolg der Forstbetriebe. Durch die Umsetzung naturschutzfachlicher Maßnahmenplanungen (z.B. Erhaltungs- und Entwicklungsmaßnahmen in FFH-Gebieten) können sich Einschränkungen der Waldbewirtschaftung und Entscheidungsfreiheiten, aber auch wirtschaftliche Verluste für Forstbetriebe ergeben. ROSENKRANZ et al. (2014) ermittelten die naturalen und ökonomischen Auswirkungen von FFH-Maßnahmenplanungen in Buchenwald-Lebensraumtypen auf 21 Fallbeispielsforstbetriebe im laufenden FFH-Umsetzungsprozess mit dem StRUGHOLTZ-EngleRT-Simulationsmodell. Die am stärksten von den Betrieben als Einschränkung empfundenen FFH-Maßnahmen waren die Ausweisung von Habitatbäumen, Ein- 
schränkungen in der Baumartenwahl und die Erhöhung des Altholzanteils. Die Deckungsbeitragsverluste, einschließlich erhöhter Verwaltungskosten, zur Umsetzung dieser Maßnahmen beliefen sich in diesen Betrieben auf durchschnittlich $40 € / \mathrm{ha} / J a h r$. Haupteinflussfaktoren der Ergebnisse waren die Altersklassenverteilung auf den Buchenwald-Lebensraumtypflächen, die Betriebsziele und die Waldbewirtschaftungspraktiken der Betriebe.

Derzeit werden Forderungen zu einer Segregation der Waldfunktionen auf Teilflächen in Deutschland laut (HöLTERMANN 2013). Dies würde eine Abkehr vom bisherigen forstpolitischen Leitbild der multifunktionalen Waldbewirtschaftung bedeuten. Die naturalen und wirtschaftlichen Auswirkungen einer möglichen Umsetzungsvariante der Segregation der Waldfunktionen auf Teilflächen, wurden von ROSENKRANZ und SEINTSCH (2015) erstmalig für die gesamte deutsche Forstwirtschaft mit dem STRUGHOLTZ-ENGLERT-Simulationsmodell berechnet. Würden die naturschutzfachlichen Maßnahmen, wie unter den in der Studie getroffenen Annahmen umgesetzt, so würden sich Holzeinschlagsverluste von rund 11,2 Mio. Efm/Jahr und Deckungsbeitragsverluste von rund $1 \mathrm{Mrd}$. $€ / J a h r$ für die deutsche Forstwirtschaft ergeben.

Die Berechnungen von RosenKRANZ et al. (2014) und ROSENKRANZ und SEINTSCH (2015) zeigen deutliche Ertragseinbußen für die Forstwirtschaft durch die Umsetzung von naturschutzfachlichen Maßnahmenplanungen, die durch staatliche Transferzahlungen ausgeglichen werden müssten. Zur Berechnung von Einschlags- und Deckungsbeitragsveränderungen wurde das STRUGHOLTZ-ENGLERTSimulationsmodell weiterentwickelt und erfolgreich angewendet. Die derzeitige Modellversion liefert plausible Ergebnisse, lässt jedoch noch Raum für weitere Verbesserungsansätze, wie z.B. die Integration von dynamischen klimasensitiven Anpassungsmöglichkeiten und von Holzpreisänderungen im Laufe der 200-jährigen Simulationsperiode.

Dem durch die Umsetzung naturschutzfachlicher Forderungen reduzierten inländischen Holzeinschlag steht die stetig steigende Nachfrage nach Holz zur energetischen und stofflichen Verwendung gegenüber. SEINTSCH und ROSENKRANZ (2014) entwickelten eine einfache Methode zur Ermittlung durchschnittlicher Kennzahlen für die holzbasierte Wertschöpfung und Beschäftigung der ersten und zweiten Holzverarbeitungsstufe. Anhand dieser Kennzahlen kann abgeschätzt werden, welche Wertschöpfung und Beschäftigung durch den Einsatz einer durchschnittlichen Einheit holzbasierter Vorleistungen in den einzelnen Wirtschaftszweigen sowie in holzbasierten Wertschöpfungsketten generiert wird und wie sich veränderter Holzeinschlag auf die Holzwirtschaft auswirkt. Durch die Übertragung der in der Studie von SEINTSCH und ROSENKRANZ (2014) ermittelten Kennzahlen auf zwei Fallbeispielsregionen konnten die Auswirkungen verminderten Holzaufkommens für verschiedene Waldbehandlungs- und Holzverwendungsszenarien für Regionen aufgezeigt werden. 
Im Rahmen einer Dekompositionsanalyse bewerteten ROSENKRANZ et al. (2015) Wertschöpfungseffekte in der Säge- und der Holzwerkstoffindustrie. Hierfür wurden Wertschöpfungsänderungen zwischen jeweils zwei Betrachtungsjahren in den Produktpreis-, den Vorleistungspreis-, den Wachstums- und den Struktureffekt zerlegt. Ein besonderer Fokus wurde auf den Einfluss von Preis- und Mengenänderungen holzbasierter Vorleistungen gelegt. In der Sägeindustrie konnte, unter Ausschluss von Preisänderungen, ein Zusammenhang zwischen dem Holzinput und dem Wachstum nachgewiesen werden. Weiterhin konnte die hohe Bedeutung von Rohholz und holzbasierten Vorleistungen für die Sägeindustrie aufgezeigt werden.

Eine Verknappung des inländischen Holzeinschlags dürfte sich negativ auf Wachstum und Wertschöpfung der Holzindustrie auswirken: zum Beispiel durch eine Erhöhung der Transportkosten, der Rohholzkosten oder durch Änderungen der Mengen- und Sortimentsstruktur von Rohholz. Kapazitätsanpassungen und Betriebsschließungen in der ersten Holzverarbeitungsstufe sind wahrscheinliche Folgen. Aufgrund der Vernetzung der holzverarbeitenden Industrie ist weiterhin zu vermuten, dass sich eine Reduktion des Holzeinschlags auch auf Wertschöpfung und Beschäftigung der zweiten Holzverarbeitungsstufe auswirkt.

Die volkswirtschaftliche Bedeutung des Clusters Forst und Holz ist vergleichsweise gering. Daher wären aus volkswirtschaftlicher Sicht die Opportunitätskosten für den Naturschutz möglicherweise tragbar. Es ist jedoch zu erwarten, dass sich Kapazitätsanpassungen und Betriebsschließungen negativ auf regionale Beschäftigungssituationen und damit indirekt auch (geringfügig) auf die Nachfrage nach anderen Gütern in Deutschland auswirken werden.

Der Fokus dieser Arbeit lag auf den wirtschaftlichen Auswirkungen der Umsetzung von Naturschutzmaßnahmen auf die Forst- und Holzwirtschaft. Für eine vollständige Kosten-Nutzenbewertung bedarf es aber auch Nutzenbewertungen von naturschutzfachlichen Maßnahmen. Studien zum Nutzen von naturschutzfachlichen Maßnahmen wurden im Rahmen dieser Arbeit jedoch nicht durchgeführt bzw. lagen nicht vor. Hier herrscht weiterer Forschungsbedarf. 


\begin{tabular}{|c|c|}
\hline$€$ & Euro \\
\hline a & Jahr \\
\hline BfN & Bundesamt für Naturschutz \\
\hline BGH & Bundesgerichtshof \\
\hline BMEL & Bundesministerium für Landwirtschaft und Ernährung \\
\hline BVerwG & Bundesverwaltungsgericht \\
\hline BWI & Bundeswaldinventur \\
\hline d.h. & das heißt \\
\hline Dr. & Doktor \\
\hline Efm & Erntefestmeter \\
\hline $\mathrm{FFH}$ & Flora-Fauna-Habitat (im Kontext von FFH-Gebiet oder FFH-Richtlinie) \\
\hline ha & Hektar \\
\hline HB & Holzbodenfläche \\
\hline $\mathrm{m}$ & Meter \\
\hline $\mathrm{m}^{2}$ & Quadratmeter \\
\hline $\mathrm{m}^{3}$ & Kubikmeter \\
\hline MCPFE & Ministerkonferenz zum Schutz der Wälder in Europa \\
\hline Mio. & Millionen \\
\hline Mrd. & Milliarden \\
\hline Prof. & Professor \\
\hline StBA & Statistisches Bundesamt \\
\hline $\mathrm{t}$ & Tonne \\
\hline TAZ & Tageszeitung \\
\hline TBN Forst & Testbetriebsnetz Forst \\
\hline u.a. & unter anderem \\
\hline vs. & versus \\
\hline WWF & World Wide Fund for Nature \\
\hline
\end{tabular}






\section{Einführung in die wissenschaftliche Fragestellung}

\subsection{Steigende Nutzungskonkurrenzen um Waldflächen und Rohholz}

Neben dem Eigenwert des Waldes als Ökosystem erfüllen Wälder viele wichtige Funktionen für die Gesellschaft. Primär sind dies die Nutz,- Schutz- und Erholungsfunktionen. Zu den Nutzfunktionen zählt die Rohstoff-, die Einkommens- und die Beschäftigungsfunktion, beispielsweise durch den Einschlag von Holz zur stofflichen und energetischen Nutzung oder den Ertrag durch weitere Walderzeugnisse. Die Schutzfunktionen des Waldes umfassen unter anderem den Klima-, Boden-, Wasserund Immissionsschutz sowie den Lärm-, Sicht- und Lawinenschutz. Darüber hinaus dient der Wald einer Vielzahl von Tier- und Pflanzenarten als Lebensraum und bietet dem Menschen vielfältige Möglichkeiten zur Freizeit- und Erholungsnutzung. Aufgrund der Nutzfunktionen des Waldes und wegen seiner Bedeutung für die Umwelt sind die Erhaltung, Mehrung und Sicherung des Waldes durch eine ordnungsgemäße Bewirtschaftung in §1 (1) des Bundeswaldgesetzes verankert.

Aus den Nutz-, Schutz und Erholungsfunktionen lassen sich gesellschaftliche Nutzungsansprüche ableiten, die zu Interessenskonflikten führen können. In den letzten Jahren sind die gesellschaftlichen Anforderungen an den deutschen Wald stark gestiegen. Den begrenzten Produktionsmöglichkeiten des Waldes steht eine Vielzahl unterschiedlicher, teilweise konkurrierender Ansprüche gegenüber. Dadurch entstehen Nutzungskonkurrenzen bezüglich der Verwendung (Allokation) und Verteilung (Distribution) der knappen Ressourcen Wald und Holz, die geregelt werden müssen. Die Abwägung und der Ausgleich der Ansprüche der verschiedenen Interessensgruppen ist ein politischer Prozess. Hierauf aufbauend müssen Regelungen durch ordnungsrechtliche oder marktliche Steuerungssysteme geschaffen werden.

Die vielfältigen Nutzungsansprüche an Waldflächen und Rohholz werden in teils nicht vollkommen widerspruchsfreien Politikprogrammen, zur Umsetzung von Zielsetzungen der Forst-, Naturschutz-, Landwirtschafts-, Klima-, Raumordnungs-, Energie-, Struktur- und Wirtschaftspolitik, aufgegriffen. Beispielsweise werden zur Verwirklichung naturschutzpolitischer Ziele Nutzungsextensivierungen und Stilllegungsflächen angestrebt (z.B. FFH-Richtlinie (92/43/EWG), Biodiversitätsstrategie (BMU 2007)) und gleichzeitig im Rahmen wirtschafts- und energiepolitischer Zielsetzungen die Steigerung sowohl der energetischen als auch der stofflichen Holznutzung forciert (z.B. Charta für Holz (BMELV 2004), Biomasseaktionsplan (BMELV und BMU 2010)). Diese Entwicklungen spiegeln sich unter anderem in der Zunahme der Waldschutzgebietsausweisungen und in der Steigerung des Holzeinschlags aufgrund erhöhter Nachfrage seitens der Holzverwender, auch verstärkt durch die Förderung erneuerbarer Energien oder durch öffentliche Investitionshilfen, wider (WEIMAR 2014; FOREST EUROPE 2011). 
Der erhöhten Nachfrage nach Holz stehen weitgehend abgeschöpfte Nutzungspotentiale, insbesondere im Nadelholz, des deutschen Waldes gegenüber (SEINTSCH 2011, DIETER und SEINTSCH 2012).

Unter den vielen verschiedenen gesellschaftlichen Nutzungskonflikten waren und sind die Nutzungskonkurrenzen zwischen Holznutzung (Forstwirtschaft und Holzwirtschaft) und Nutzungsverzicht (Naturschutz) in den letzten Jahren das bestimmende Thema und damit von zentraler Bedeutung. Dies wird in zahlreichen Pressemitteilungen, auf Internetseiten von Bürgerbewegungen, Parteien und Verbänden und in Veröffentlichungen deutlich ${ }^{1}$. Die Bewertung dieser Nutzungskonkurrenzen ist daher Thema dieser Arbeit.

\subsection{Problemaufriss}

\subsubsection{Zur Situation der Forstwirtschaft in Deutschland}

Die Waldfläche in Deutschland beträgt aktuell 11,4 Mio. ha (BWI 2012a). Die Eigentumsarten des deutschen Waldes umfassen den Staatswald (Bundes-, Landes- und Treuhandwald), den Körperschaftswald und den Privatwald (BWALDG §3). Dabei hat der Bundeswald einen Anteil von $4 \%$, der Landeswald von 29 \%, der Körperschaftswald von $19 \%$ und der Privatwald von $48 \%$ an der deutschen Waldfläche. (BWI 2012). Insgesamt werden also 52 \% der Waldfläche Deutschlands durch die öffentliche Hand und $48 \%$ privat bewirtschaftet.

In der Forstwirtschaft werden durch planmäßiges Handeln (=wirtschaften) materielle Leistungen, wie die Rohholzerzeugung sowie immaterielle Leistungen, wie die Erhaltung der Wälder als Schutz- und Erholungsraum für die Gesellschaft erbracht (ERLBECK et al. 1998). Während es sich bei den Nutzfunktionen, insbesondere bei der Rohholzproduktion, überwiegend um private Güter handelt, haben Schutz- und Erholungsfunktionen eher den Charakter öffentlicher Güter (vgl. MöHRING und MESTEMACHER 2002, OESTEN und ROEDER 2002). Der wirtschaftliche Erfolg von Forstbetrieben hängt primär von der Erzeugung und Veräußerung des privaten Gutes Rohholz ab. So hatte beispielsweise der erzielte Erlös aus dem Holzverkauf der Betriebe des Testbetriebsnetzes Forst des BMEL ${ }^{2}$ in den Jahren 2003 bis 2011 durchschnittlich einen Anteil von rund 80 \% am Ertrag des Produktbereiches 1 „Produktion von Holz und andere Erzeugnisse“ und rund $70 \%$ am Gesamtertrag (ERMISCH et al. 2013) ${ }^{3}$.

Im Zeitraum 2003 bis 2012 wurden in Deutschland über alle Waldbesitzarten jährlich rund 76 Mio. Efm Holz eingeschlagen (BWI 2012). Nach Auswertungen des Testbetriebsnetzes Forst wurde die Einschlagsentwicklung der letzten Jahre insbesondere geprägt durch das Sturmereignis Kyrill im Jahr 2007, mit einem hohen Anfall nicht geplanter Holzmengen, sowie dem sinkenden Einschlag in den

\footnotetext{
${ }^{1}$ z.B. Krott et al. 2000, Forstmaschinenprofi 2010, Stuttgarter Zeitung 2011, Bürgerbewegung Unser Teutoburger Wald 2012, Greenpeace 2012, TAZ, 2013, WWF 2013

${ }^{2}$ Rund 360 Forstbetriebe, davon rund 10 Staatswald-, 225 Körperschaftswald- und 125 Privatwaldbetriebe melden an das Testbetriebsnetz Forst des BMEL. Es handelt sich um Betriebe mit mehr als 200 ha Waldfläche.

${ }^{3}$ Berechnungsgrundlage: Durchschnittliche Einnahmen aus Einschlägen des Berichtsjahres und des Vorjahres, ohne Selbstwerbereinnahmen
} 
Folgejahren, bedingt durch den Ausgleich der Kalamitätsnutzungen des Sturmjahres und die Wirtschaftskrise im Jahr 2009. Höhere Einschläge im Jahr 2010 sind auf die hohe energetische Nutzung, bedingt durch den kalten Winter, und die Erholung nach der Wirtschaftskrise zurückzuführen. In den Jahren 2011 und 2012 blieb der Einschlag konstant (ERMISCH et al. 2014, SEINTSCH und ROSENKRANZ 2014, JOCHEM et al. 2015a).

Der Produktionswert des Wirtschaftsbereichs Forstwirtschaft ${ }^{4}$ lag im Jahr 2012 bei 4,8 Mrd. € und der Nettounternehmensgewinn bei 1,34 Mrd. €, was einem Anteil von rund $28 \%$ am Produktionswert entspricht (SEINTSCH und ROSENKRANZ 2014). Bei einer rückblickenden Betrachtung der Produktionswerte seit dem Jahr 1991 wird jedoch deutlich, dass die Gewinne im Wirtschaftsbereich Forstwirtschaft ein eher kurzfristiges Phänomen sind: erst seit dem Jahr 2005 wurden hier Nettounternehmensgewinne verzeichnet. Davor war der Wirtschaftsbereich Forstwirtschaft durch Unternehmensverluste oder nur geringe Gewinne geprägt. (SEINTSCH 2011, SEINTSCH und ROSENKRANZ 2014). Grund für die hohen Nettounternehmensgewinne seit 2005 sind neben Kosteneinsparungen und Rationalisierungen (z.B. Strukturreformen, Personalabbau) insbesondere die Einschlagshöhe und die in den letzten Jahren gestiegenen Rohholzpreise (DIETER und BORMANN 2009, SEINTSCH 2011).

Ein Leitbild der Forstwirtschaft war lange Zeit die Kielwassertheorie. Sie besagt, dass die Schutz- und Erholungsfunktionen ohne weiteres Zutun im „Kielwasser“ der Nutzfunktionen des Waldes erbracht werden (RUPF 1961). Im Zuge der steigenden gesellschaftlichen Ansprüche in den vergangenen Jahren wurde jedoch der Vorrang der Nutzfunktion als nicht mehr haltbar erachtet (HANEWINKEL 2011). Das derzeit vorherrschende forstpolitische Leitbild ist die multifunktionale Forstwirtschaft (nach DIETERICH 1953). Dieser weit verbreitete, integrative Ansatz ist bestrebt, die Nutz-, Schutz- und Erholungsfunktionen auf der gleichen Fläche bereitzustellen. SUDA und PUKALL (2014) sprechen beispielsweise von einer Integration der unterschiedlichen Ansprüche auf der gleichen Waldfläche im Zuge der bayerischen Waldnutzung, die zwar Konflikte hervorruft, die aber durch entsprechende Schwerpunktbildung bei der Waldbewirtschaftung gelöst werden können. Der Ansatz der integrativen, multifunktionalen Waldwirtschaft wurde zuletzt in der Waldstrategie 2020 des BMEL herausgestellt und soll fortgeführt werden (BMELV 2011). Durch steigende Bedürfnisse einzelner Interessensgruppen wird das Konzept der Multifunktionalität zukünftig jedoch nur noch als bedingt tragfähig angesehen (vgl. THOROE 2009, BORCHERS 2010, HANEWINKEL 2011). So können einige (naturschutzfachliche) Ansprüche, wie zum Beispiel eine großflächige Urwaldentwicklung, durch die multifunktionale Waldwirtschaft objektiv nicht erfüllt werden. Nicht zuletzt aus diesem Grund wurde daher im Zuge der steigenden Anforderungen zur Anhebung des Waldnaturschutzniveaus in den letzten Jahren die flächige Funkti-

\footnotetext{
${ }^{4}$ Der Wirtschaftsbereich Forstwirtschaft entspricht der Wirtschaftszweig-Abteilung 02 Forstwirtschaft und Holzeinschlag der amtlichen Klassifikation der Wirtschaftszweige (WZ 2008). Diese WZ-Abteilung umfasst die Wirtschaftszweig-Gruppen 02.1 Forstwirtschaft, 02.2 Holzeinschlag, 02.3 Sammeln von wildwachsenden Produkten (ohne Holz) und 02.4 Erbringung von Dienstleistungen für Forstwirtschaft und Holzeinschlag (StBA 2007).
} 
onentrennung (Segregation) diskutiert. So wird beispielsweise derzeit seitens des Naturschutzes, unter der Bezeichnung „Neue Multifunktionalität“, eine Segregation der Waldfunktionen auf Teilflächen vorgeschlagen (HÖLTERMANN 2013).

Das Eigentum und damit die „Privatnützlichkeit“ des Eigentums natürlicher und juristischer Personen des Privatrechts wird durch Artikel 14 des Grundgesetzes geschützt (SCHMIDT-BLEIBTREU et al. 2011). In $\S 14$ Absatz 2 wird ergänzt, dass das Eigentum zugleich (also nicht vorrangig) dem Wohle der Allgemeinheit dienen soll (Sozialpflichtigkeit des Eigentums). Diese Sozialpflichtigkeit des Privateigentumswird durch die Waldgesetzte konkretisiert. Darüber hinaus wird in den Waldgesetzen die öffentliche Hand gebunden, indem gefordert wird, dass der öffentliche Wald insbesondere dem Allgemeinwohl zu dienen hat.

Zwischen den verschiedenen Eigentumsarten bestehen unterschiedliche Zweckbindungen und Bewirtschaftungsziele. Gemäß KLOSE und ORF (1998) werden dem öffentlichen Wald im besonderen Maße Aufgaben hinsichtlich der Bereitstellung der Schutz- und Erholungsfunktionen des Waldes zugesprochen. Folglich unterliegt der öffentliche Wald höheren Gemeinwohlverpflichtungen als der Privatwald, der, abhängig von den individuellen Eigentümerzielen, verstärkt auch erwerbswirtschaftliche Interessen verfolgen kann (KLOSE und ORF 1998, BERGEN et al. 2013). In den letzten Jahren ist jedoch zu beobachten, dass immer wieder angeregt wird, die freiwilligen Selbstverpflichtungen des öffentlichen Waldes, insbesondere hinsichtlich der Schutz- und Erholungsfunktionen, über die Sozialpflichtigkeit des Eigentums auch als Standards für die „ordnungsgemäße Bewirtschaftung“ des Privatwaldes zu etablieren (vgl. THOROE et al. 2003). Auf diese Weise würden Verfügungsrechte der Waldeigentümer zugunsten des Allgemeinwohls eingeschränkt werden (BERGEN et al. 2013).

Um die Interessen der Allgemeinheit gegenüber den Interessen des Waldeigentümers durchzusetzen stehen dem Gesetzgeber zwei Mittel zur Verfügung: die Legalenteignung (verbunden mit einer Entschädigungsregel) sowie die ausgleichspflichtige und die nicht-ausgleichspflichtige Inhaltsbestimmung des Eigentums (GRUNDGESETZ § 14(1), KLOSE und ORF, 1998). „Der BGH [Bundesgerichtshof] erkennt eine Ausgleichspflicht dann an, wenn der Eigentümer unverhältnismäßig oder im Vergleich zu anderen ungleich in unzumutbarer Weise belastet wird. Das BVerwG [Bundesverwaltungsgericht] verlangt für die Anerkennung einer Ausgleichspflicht eine unangemessene Beschränkung der Eigentümerbefugnisse, die vorliegt, wenn ohne finanziellen oder sonstigen Ausgleich eine Nutzung unterbunden wird, die bisher ausgeübt wurde oder die sich nach Lage der Dinge objektiv anbietet" (THOROE et al. 2003:7).

Die wirtschaftliche Situation der Forstwirtschaft wird maßgeblich durch die Einschlagshöhe bestimmt (DIETER und BORMANN 2009). Nutzungsextensivierungen durch naturschutzfachliche Maßnahmenplanungen können sich direkt auf die produktiven und sozioökonomische Funktionen der Wälder (DIETER 
2013), aber auch auf die aktive Bereitstellung anderer öffentlicher Güter auswirken, die maßgeblich durch Erträge der Rohholzproduktion finanziert werden. Ob die Kosten für die Umsetzung der Ansprüche der Gesellschaft zu Lasten der Waldbesitzer fallen und ob sie eine unverhältnismäßige Belastung darstellen ist, aufgrund der unterschiedlichen Strukturen und naturalen Ausstattungen der Forstbetriebe und der unterschiedlichen Maßnahmen, als Einzelfallentscheidung zu prüfen.

\subsubsection{Zur Situation der Holzwirtschaft in Deutschland}

Die Holzwirtschaft ist durch die wirtschaftliche Verwertung des Rohstoffs Holz gekennzeichnet. Zahlreiche Branchen sind in Deutschland in holzbasierte Wertschöpfungsketten eingebunden (Cluster Forst und Holz). Umsatz, Wertschöpfung und Beschäftigung dieser Branchen werden im Rahmen der Clusterstatistik Forst und Holz ${ }^{5}$ erfasst (vgl. BECHER 2014). Im Jahr 2012 wurde im Cluster Forst und Holz ein Umsatz von 181 Mrd. € erzielt. Insgesamt waren rund 1,1 Mrd. Arbeitnehmer im Cluster Forst und Holz beschäftigt. Mit einem Anteil an der deutschen Volkswirtschaft von 3,1 \% des Umsatzes, 2,2 \% der Bruttowertschöpfung und 3,0 \% der Beschäftigten im Jahr 2012 (BECHER 2014) ist die volkswirtschaftliche Bedeutung des Clusters Forst und Holz vergleichsweise gering.

Die inländische Holzverwendung zeigt zwischen 1991 und 2013 eine leicht steigende Tendenz. Im Jahr 2013 lag der inländische Holzverbrauch, ohne Export und Lagerbestände, bei rund 74,7 Mio. $\mathrm{m}^{3}$ Rohholz (JOCHEM et al. 2015b). Die Nadelrohholznutzung liegt in Deutschland weit über der Laubrohholznutzung. Im Jahr 2013 basierte die inländische Rohholzverwendung zu rund $75 \%$ aus Nadelholz und zu $25 \%$ aus Laubholz. Das Laubholz wird zu gut zwei Dritteln der energetischen Verwendung zugeführt (JOCHEM et al. 2015b). Größtenteils wird in der Holzindustrie der Bundesrepublik Deutschland inländisches, mittelstarkes Nadelholz verarbeitet. Die stofflichen Verwender sind zu rund $90 \%$ auf Nadelholz angewiesen (SEINTSCH und ROSENKRANZ 2015b).

Die bedeutendsten rohholzaufnehmende Branchen der stofflichen Verwendung sind die Sägeindustrie, die Holzwerkstoffindustrie sowie die Holz- und Zellstoffindustrie. Sie werden auch als erste Holzverarbeitungsstufe bezeichnet. Wichtige Branchen der zweiten Holzverarbeitungsstufe sind unter anderem die Möbelindustrie, der Holzbau und die Papierindustrie. Aufgrund der hohen Transportkostenanfälligkeit von Rohholz ist die Nähe zur Rohstoffbasis für die Unternehmen der ersten Holzverarbeitungsstufe ein wichtiger Standortfaktor (HELD et al. 2008, HARSCHE und JAENSCH 2009). Die Beschaffungsmärkte der ersten Holzverarbeitungsstufe sind daher maßgeblich lokal oder regional. Beim Absatz sind die Unternehmen der ersten Holzverarbeitungsstufe jedoch ebenfalls den Bedingungen des Weltmarktes ausgesetzt und davon abhängig.

\footnotetext{
${ }^{5}$ Das Cluster Forst- und Holz beinhaltet die Forstwirtschaft, das holzbearbeitende Gewerbe (Säge-, und Holzwerkstoffindustrie), das holzverarbeitende Gewerbe (Möbelindustrie, Holzpackmittelindustrie, Holzbauwesen und sonstige holzverarbeitenden Unternehmen), das Papiergewerbe (Holz- und Zellstoffindustrie, Papierherstellung und Papierverarbeitung), das Holz im Baugewerbe, das Verlags- und Druckwesen und den Holzhandel.
} 
Starke Schwankungen des Umsatzes und der Bruttowertschöpfung spiegeln die konjunkturell ereignisreiche Zeit zwischen 2002 und 2012 für die Holzwirtschaft wider. Der Zeitraum 2002 bis 2006 war durch Kapazitätssteigerungen sowie starke Wachstumsraten und Verkaufsmengen, bedingt durch aufnahmefähige nationale und internationale Märkte, gekennzeichnet (DIETER 2005, 2006, 2007; SEINTSCH 2010, 2011; SEINTSCH und WEIMAR 2013). Dadurch konnten die internationale Wettbewerbsfähigkeit und die Auslandsumsätze gesteigert werden (DIETER 2009; DIETER und ENGLERT 2007, 2009; StBA F7 R2; OCHS et al. 2007; LÜCKGE et al. 2008). Insgesamt führten diese Entwicklungen zu einer guten wirtschaftlichen Situation der Unternehmen der ersten Holzverarbeitungsstufe: Im Jahr 2006 betrug der Anteil der Bruttoproduktionsgewinne am Bruttoproduktionswert in der Sägeindustrie 5,4\%, in der Holzwerkstoffindustrie 1,4\% und in der Holz- und Zellstoffindustrie 6,9\% (SEINTSCH und ROSENKRANZ 2015a).

Zur gleichen Zeit stieg aufgrund steigender Energiepreise und energiepolitischen Förderungen jedoch auch der Bedarf nach Holz zur energetischen Nutzung. Dies führte zu intensiven Beschaffungskonkurrenzen, insbesondere um Industrieholz oder Sägenebenprodukte, sowohl zwischen den holzverarbeitenden Branchen als auch zwischen den stofflichen und energetischen Verwendern (OCHS et al. 2007; MANTAU 2010). In der Folge der genannten Entwicklungen stiegen sowohl die durchschnittlichen Holzpreise als auch der Holzeinschlag (ERMISCH et al. 2013, STBA F17 R 2). Die Preisentwicklung setzte sich nach 2006 zunächst fort (ERMISCH et al. 2013, STBA F17 R 2). Auch das inländische Holzaufkommen stieg zunächst bis zum Jahr 2007 aufgrund hoher Nachfrage und Sturmereignissen. Mit der Weltwirtschaftskrise von 2008 bis 2009 ging die in- und ausländische Nachfrage nach Holzhalb- und Holzfertigwaren jedoch stark zurück und der Holzmarkt brach ein (MANTAU 2010; SEINTSCH 2010; SEINTSCH und WEIMAR 2013). Diese Entwicklung zeigt die starke Abhängigkeit der deutschen Holzwirtschaft vom Weltmarkt. Als Reaktion auf die Wirtschaftskrise wurden die Kapazitäten der holzbearbeitenden und holzverarbeitenden Industrie teilweise reduziert. Dagegen blieben die hohe Nachfrage nach Energieholz sowie die Holzpreissteigerungen weiterhin bestehen (HELD et al. 2008; MANTAU 2010 \& 2012). In den Unternehmen der ersten Holzverarbeitungsstufe äußerte sich dies durch einen Rückgang der Bruttoproduktionsgewinne hauptsächlich aufgrund höherer Ausgaben für Vorleistungen. In der Holzwerkstoffindustrie überstieg im Jahr 2010 die Abgeltung der Vorleistungen und eigenen Produktionsfaktoren den Bruttoproduktionswert, was zu Unternehmensverlusten führte (SEINTSCH und ROSENKRANZ 2015a).

Infolge des stetig steigenden Bedarfs nach Holz für die energetische Nutzung und den daraus folgenden Beschaffungskonkurrenzen konnten insbesondere zwei Entwicklungen beobachtet werden. Einerseits konnte die Sägeindustrie vom Preisanstieg der vormalig niedrig notierten Sägenebenprodukte, wie z.B. Sägemehl zur Produktion von Holzpellets, profitieren. Auf der anderen Seite sahen sich die Holzindustriezweige, die auf (vormals kostengünstiges) Industrieholz oder günstige Sägeneben- 
produkte angewiesen sind, plötzlich starken Preissteigerungen und hohem Konkurrenzdruck um das benötigte Rohmaterial gegenüber (MANTAU 2010).

Die Nutzungskonkurrenzen zwischen stofflicher und energetischer Holznutzung werden unter anderem auch durch verschiedene energiepolitische Programme, wie z.B. dem Aktionsprogramm „Energie für morgen - Chancen für ländliche Räume“ des BMELV (2009), dem Gesetz zur Förderung Erneuerbarer Energien im Wärmebereich (Bundestag 2009) und dem Nationalen Biomasseaktionsplan des BMELV und BMU (2010) verstärkt. Dem generellen Anstieg des Holzbedarfs steht gegenüber, dass der Anteil des produktiven Nadelholzes an der deutschen Waldfläche zwischen 2002 und 2012, aufgrund des Waldumbaus hin zu höheren Laubholzanteilen, um 4,3\% gesunken ist (BWI 2012c). Gleichzeitig wird die Nutzung von Waldflächen durch die Umsetzung naturschutzfachlicher Maßnahmen weiter extensiviert.

\subsubsection{Zur Situation des Waldnaturschutzes in Deutschland}

Ziele des Naturschutzes in Deutschland sind der Schutz und die dauerhafte Sicherung der biologischen Vielfalt, der Leistungs- und Funktionsfähigkeit des Naturhaushalts sowie der Vielfalt, Eigenart, Schönheit und des Erholungswerts von Natur und Landschaft. Dies umfasst unter anderem die Erhaltung von wild lebenden Tieren und Pflanzen und deren Lebensstätten sowie den Schutz von Lebensgemeinschaften und Biotopen ( $\S 1(1$ und 2$)$ BNatSchG). Schutzgebietsausweisungen, wie zum Beispiel von Natur- und Landschaftsschutzgebieten, Nationalparken, Biosphärenreservaten oder Natura 2000-Gebieten, leisten einen wichtigen Beitrag zur Erreichung dieser Ziele.

\section{Waldschutzgebiete in Deutschland}

Zwischen den Jahren 2000 und 2010 hat in Deutschland der Anteil der Biodiversitätsschutzgebiete mit minimaler menschlicher Beeinflussung (MCPFE Class 1.2) um $142 \%$ und der Biodiversitätsschutzgebiete mit aktivem Management (MCPFE Class 1.3) um 51 \% zugenommen (FOREST EUROPE 2011). Nach POLLEY (2009) liegen insgesamt rund zwei Drittel der Wälder Deutschlands in Schutzgebieten, der Großteil davon im Bundeswald und den Landeswäldern. Dies dürfte überwiegend auf die hohen Gemeinwohlverpflichtungen der Waldeigentumsarten in öffentlicher Hand zurückzuführen sein. Insgesamt sind $26 \%$ der Wälder mit intensiven (z.B. Nationalparks, Biosphärenreservate, Naturschutzgebiete und Natura 2000-Gebiete) und $41 \%$ mit extensiven Schutzgebietskategorien (Landschaftsschutzgebiete, Naturparke) belegt (POLLEY 2009).

Die Zunahme der Schutzgebietsfläche dürfte größtenteils den Ausweisungen von Natura 2000Gebieten seit dem Jahr 2004 sowie, in jedoch eher geringem Maße, der Ausweisung neuer Nationalparke (z.B. Nationalpark Eifel und Nationalpark Kellerwald-Edersee im Jahr 2004; Nationalpark Harz im Jahr 2006) geschuldet sein (BFN 2009, 2012, 2015). 


\section{Das europäische Schutzgebietsnetz Natura 2000}

Das Schutzgebietsnetz Natura 2000 basiert auf der Richtlinie 92/43/EWG des Rates der Europäischen Gemeinschaften vom 21. Mai 1992 zur „Erhaltung der natürlichen Lebensräume sowie der wildlebenden Tiere und Pflanzen“ (FAUna-FLoRA-HABITAT-RICHTLINIE, kurz: FFH-RICHTIINIE) und der Richtlinie des Rates vom 2. April 1979 über die „Erhaltung der wildlebenden Vogelarten 79/409/EWG“ (VOGELSCHUTZRICHTLINIE). Mit rund $24 \%$ der Waldfläche haben Natura 2000-Gebiete hinter den Landschaftsschutzgebieten (47\%) und Naturparken (38\%) den drittgrößten Anteil an der deutschen Waldschutzgebietskulisse. Von den intensiven Schutzgebietskategorien haben Natura 2000-Gebiete den größten Flächenanteil im Wald (POLLEY 2009).

Im Zuge der Umsetzung von Natura 2000 in Deutschland wurden 1,8 Mio. ha Waldfläche zu FFHGebieten (BFN 2012) erklärt. Die FFH-Richtlinie weist als Schutzobjekte Wald-Lebensraumtypen aus. Insgesamt gibt es in Deutschland 18 Wald-Lebensraumtypen. Von der FFH-Waldfläche in Deutschland sind rund 817.000 ha bzw. $46 \%$ als Wald-Lebensraumtypen ausgewiesen. Die flächenmäßig bedeutsamsten sind, mit zusammen rund 586.000 ha, der Hainsimsen-Buchenwald (Code 9110) und der Waldmeister-Buchenwald (Code 9130). Die restlichen 54 \% der FFH-Waldfläche sind Füll- und Pufferflächen, die entweder keinen besonderen Schutzstatus haben oder als Flächen zum Schutz bestimmter Pflanzen- und Tierarten sowie als Flächen zum Schutz anderer Lebensraumtypen wie u.a. Quellen, Felsen und Magerrasen dienen (BFN 2012, ROSENKRANZ et al. 2012).

\section{Naturschutzfachliche Maßnahmenplanung in FFH-Gebieten}

Gemäß Artikel 6 der FFH-Richtlinie sind die EU-Mitgliedsstaaten verpflichtet, geeignete Maßnahmen für die einzelnen Schutzgebiete zu treffen, die einer Verschlechterung der Erhaltungszustände der Lebensraumtypen und Habitate sowie erheblichen Störungen von Arten entgegenwirken. Diese Maßnahmen sind in Bewirtschaftungsplänen (Managementplänen) festzulegen und in geeigneter Form rechtlich, administrativ oder vertraglich umzusetzen (EUROPÄISCHE KOMMISSION 2003).

Grundlage für die Festlegung der Erhaltungs- und Entwicklungsmaßnahmen ist die Erhaltungszustandsbewertung der FFH-Gebiete. Die Bewertung des Erhaltungszustands von Lebensraumtypen erfolgt nach einem zweistufigen Verfahren (BURKHARDT et al. 2004). Zunächst werden die drei Kriterien „Habitatstrukturen“, „Lebensraumtypisches Arteninventar" und „Beeinträchtigungen“, auf Basis bestimmter Parameter und Schwellenwerte, jeweils mit den Wertstufen A, B oder C bewertet. Die Bewertungen der drei gleichwertigen Kriterien werden dann zu einem Gesamtwert zusammengefasst (BURKHARDT et al. 2004). Der Gesamtwert A bezeichnet einen sehr guten Erhaltungszustand, der Gesamtwert $B$ einen guten Erhaltungszustand und der Gesamtwert $C$ einen mittleren bis schlechten Erhaltungszustand. Bei einer Analyse der Bewertungsschemata der Bundesländer (mit Ausnahme der Stadtstaaten) wurde deutlich, dass die Schwellenwerte zur Ermittlung des Erhaltungszustands in den 
einzelnen Bundesländern in einigen Fällen weit voneinander abweichen. Beispielhaft seien hier die Schwellenwerte für Totholz in den Lebensraumtypen "9110 Hainsimsen- Buchenwald“ und "9130 Waldmeister-Buchenwald" genannt: Für den Erhaltungszustand B werden in Niedersachsen, Nordrhein-Westfalen, Saarland und Sachsen 1 bis 3 Stück Totholz pro Hektar, dagegen in Hessen 5 bis 14 $\mathrm{m}^{3}$ /ha und in Brandenburg 21 bis $40 \mathrm{~m}^{3} /$ ha gefordert (ROSENKRANZ et al. 2012, ROSENKRANZ und WIPPEL 2012).

Auf Basis des Erhaltungszustands der FFH-Gebiete werden von den Bundesländern in FFHManagementplänen Erhaltungs- und Entwicklungsmaßnahmen für die jeweiligen Gebiete festgelegt. Diese werden in der Studie von RosenKRANz et al. (2012:63), auf Grundlage einer Auswahl von $44 \mathrm{FFH}$ Managementplänen, folgendermaßen definiert: Erhaltungsmaßnahmen sind Maßnahmen, die als nötig erachtet werden den gegenwärtigen Erhaltungszustand zu bewahren. Entwicklungsmaßnahmen hingegen sind Maßnahmen, die den Erhaltungszustand verbessern oder Lebensraumtypflächen im FFH-Gebiet vergrößern. ROSENKRANZ et al. (2012) fanden in FFH-Managementplänen für die beiden flächenbedeutsamsten Lebensraumtypen "9110 Hainsimsen-Buchenwald" und "9130 WaldmeisterBuchenwald" folgende, häufig genannte Erhaltungsmaßnahmen:

- Naturnahe Waldbewirtschaftung

- Erhalt/Erhöhung des Anteils von Habitatbäumen und Totholz

- Erhalt/Erhöhung des Anteils von Altholz/ Bäumen in der Reifephase

- Erhalt der lebensraumtypischen Artenzusammensetzung

- Förderung standortheimischer Arten bei der Waldpflege

- Erhalt/Förderung seltener und begleitender Baumarten

- Zurückdrängen/sukzessive Entnahme nicht standortgerechter Baumarten, dauerhafte Beschränkung des Mischungsanteils

- Förderung der Naturverjüngung standortheimischer Baumarten

- Einzelstammweise Zielstärkennutzung oder Femelhiebe

- Strukturfördernde Maßnahmen

- Befahrung nur auf Rückegassen

- Einsatz bodenschonender Rücketechniken

Die europäische FFH-Richtlinie wurde im Jahr 1998 ins Bundesrecht umgesetzt (SIPPEL 2007). Sie ist in den $\S \S 32$ bis 38 des Bundesnaturschutzgesetzes verankert. Für die Umsetzung und Konkretisierung der in der FFH-Richtlinie geforderten Maßnahmen sowie für die Sicherstellung der FFH-Gebiete sind die Bundesländer zuständig. Während die Gebietsausweisung inzwischen weitgehend abgeschlossen ist, war die Erstellung der Managementpläne im Jahr 2011 noch im Anfangsstadium (SEINTSCH et al. 2012). 


\section{Auswirkungen von FFH-Maßnahmenplanungen}

Die FFH-Erhaltungs- und Entwicklungsmaßnahmen können die bisherige Waldbehandlung einschränken und in Folge zu Mehraufwendungen oder Mindererträgen für Waldbesitzer führen. Nutzungskonkurrenzen zwischen Forstwirtschaft und Naturschutz sind zu erwarten. Im Rahmen einer (nicht repräsentativen) bundesweiten Online-Befragung von Forstbetrieben zu den Bewirtschaftungseinschränkungen durch FFH-Maßnahmenplanungen wurde deutlich, dass insbesondere die Maßnahmen „Belassen von Altholzresten bis zum natürlichen Zerfall“, „Altholzanteile belassen oder erhöhen“ und „Erhalt der lebensraumtypischen Waldgesellschaft“ als Belastung empfunden werden (SEINTSCH et al. 2012a, b). Die zu erwartende Reduktion von Holzerntemengen aufgrund naturschutzfachlicher Maßnahmenplanungen kann weiterhin zu Versorgungsengpässen für die Rohholzabnehmer und somit zu Nutzungskonkurrenzen zwischen Naturschutz und Holzwirtschaft führen.

\subsection{Forschungsfragen und Ziele der Arbeit}

Im Hinblick auf die derzeitigen Entwicklungen ist zu erwarten, dass in Zukunft nicht mehr alle Ansprüche an den Wald vollständig erfüllt werden können (vgl. DIETER et al. 2008). Politikfolgeabschätzungen und Effizienzbewertungen zu den Auswirkungen der Umsetzung der gesellschaftlichen Forderungen an den Wald erlangen somit eine hohe Bedeutung. Ökonomische Bewertungen können einen wichtigen Beitrag zur Entscheidungsfindung bei der Umsetzung konkurrierender Ansprüche an die Wald- und die Holznutzung leisten. Für eine umfassende Analyse der unterschiedlichen Dimensionen der naturalen und ökonomischen Auswirkungen der Umsetzung gesellschaftlicher Forderungen nach Wald- und Holznutzung sind wissenschaftliche Bewertungsansätze erforderlich. Die vorliegende Arbeit soll hierzu einen Beitrag leisten.

Vor diesem Hintergrund ist das Ziel dieser Arbeit die Bewertung der naturalen und ökonomischen Auswirkungen von Nutzungskonkurrenzen zwischen der Forst- und Holzwirtschaft (stoffliche Verwendung) einerseits und dem Naturschutz andererseits. Hierfür werden die folgenden wissenschaftlichen Fragestellungen bearbeitet:

1. Welche Auswirkungen haben aktuelle naturschutzfachliche Maßnahmenplanungen auf die Forstwirtschaft?

2. Welche Auswirkungen hat der veränderte inländische Holzeinschlag auf die Holzindustrie? Daraus lassen sich folgende Zielsetzungen ableiten:

1. Weiterentwicklung / Eignungsprüfung eines Modells zur naturalen und ökonomischen Bewertung der Auswirkungen naturschutzfachlicher Maßnahmenplanungen auf Forstbetriebe

2. Eignungsprüfung eines Modells zur naturalen und ökonomischen Bewertung von Auswirkungen der Segregation von Waldfunktionen auf Bundesebene 
3. Entwicklung und Eignungsprüfung eines Verfahrens zur Bewertung der Auswirkungen von Preis- und Mengenänderungen auf die Wertschöpfung und Beschäftigung der Holzwirtschaft

4. Entwicklung und Eignungsprüfung eines Verfahrens zur Bewertung von Wertschöpfungseffekten

\subsection{Stand des Wissens}

Zur Analyse der Auswirkungen von Nutzungskonkurrenzen zwischen Naturschutz, Forst- und Holzwirtschaft sind Bewertungen aus unterschiedlichen Blickwinkeln notwendig. Zuerst sind die naturalen und ökonomischen Auswirkungen von naturschutzfachlichen Maßnahmenplanungen auf die Forstwirtschaft zu bewerten. Hierbei ist von Interesse, welche Auswirkungen die Maßnahmen auf einzelne Forstbetriebe haben (z.B. zur Entscheidung über Kompensationsprogramme und ggf. deren Ausgestaltung) und welche Auswirkungen auf die gesamte deutsche Forstwirtschaft zu erwarten sind (z.B. zur Abschätzung des zukünftigen inländischen Holzeinschlags). In einem nächsten Schritt sind die Auswirkungen von verändertem Holzeinschlag auf die holzverarbeitende Industrie zu prüfen. Hierzu sind der Zusammenhang zwischen Holzinput, Wachstum und Bruttowertschöpfung in der Holzindustrie sowie die Wertschöpfung und Beschäftigung, die durch den Einsatz einer Einheit Rohholz generiert werden kann, zu analysieren. Anhand dieser Bewertungen kann abgeschätzt werden, welche Wertschöpfungs- und Beschäftigungsverluste durch verringerten Holzeinschlag entstehen können.

Ebenfalls von Interesse sind die Auswirkungen von Nutzungskonkurrenzen zwischen stofflicher und energetischer Holznutzung. Die energetische Nutzung von Holz ist in den amtlichen Statistiken jedoch nicht differenziert erfasst und eine vertiefende sektorale Analyse der energetischen Holznutzung ist daher nicht möglich (vgl. SCHWEINLE 2012). Eine Analyse der stofflich-energetischen Nutzungskonkurrenzen konnte daher im Rahmen dieser Arbeit nicht durchgeführt werden.

\subsubsection{Herausforderungen bei der Bewertung der Auswirkungen naturschutzfachlicher Maßnah-} menplanungen auf die Forstwirtschaft

Grundsätzlich ist zu erwarten, dass sich die Auswirkungen naturschutzfachlicher Maßnahmenplanungen auf die Forstwirtschaft auf zweierlei Weise zeigen: zum einen in Form von Mindererträgen durch Unterlassung oder Aufschub waldbaulicher Behandlungen und zum anderen in Form von Mehraufwendungen. Folglich müssen nicht nur Mehraufwendungen sondern auch die entgangenen oder nicht realisierten Erträge durch Umsetzung naturschutzfachlicher Maßnahmenplanungen auf die Forstwirtschaft bewertet werden. Mindererträge und Mehraufwendungen sind immer dann zu erwarten, wenn die geforderte Waldbehandlung von der betriebsindividuell praktizierten und/oder zukünftig geplanten Waldbewirtschaftung abweicht. Zur Bewertung der Veränderungen durch naturschutzfachliche Maßnahmenplanungen muss daher eine Waldbewirtschaftung, welche jeweilige Na- 
turschutzauflagen integriert, mit einer Referenzwaldwirtschaft verglichen werden. Von Interesse ist der Vergleich der Höhe des Einschlags/Ertrags eines Waldes ohne im Vergleich zur Höhe des Einschlags/Ertrags eines Waldes mit Naturschutzauflagen. Hierbei ist zu berücksichtigen, dass Waldbesitzer zukünftig unter Umständen derzeitige Waldbewirtschaftungsziele ändern möchten. Es könnten sich folglich Abweichungen vom Status quo ergeben. Weiterhin ist zu beachten, dass sich die naturalen und wirtschaftlichen Verluste für Forstbetriebe bei der Umsetzung von Einzelmaßnahmen oft aus einer Kombination von Mehraufwendungen und Mindererträgen ergeben (Tabelle 1). Geeignete Bewertungsmethoden sollten diese Umstände berücksichtigen.

Einen starken Einfluss dürfte, neben den waldbaulichen Zielsetzungen, auch die naturale Ausstattung (z.B. Altersklassen- und Baumartenanteile) der Betriebe haben. Für die Bewertung bedarf es dementsprechend einer Referenz, welche die naturale Ausstattung und die derzeitige Waldbehandlung des zu bewertenden Betriebes hinreichend abbildet. Um die Situation von Forstbetrieben möglichst realitätsnah abzubilden, sollen im Rahmen dieser Arbeit folglich keine Berechnungen für idealisierte Normalwaldbestände oder gleichaltrigen Bestände vom Alter Null bis zum Ende der Produktionszeit, sondern für Forstbetriebe/Betriebsteile mit ihrer spezifischen Altersklassenausstattung durchgeführt werden.

Tabelle 1: Mögliche Auswirkungen naturschutzfachlicher FFH-Maßnahmen (eigene Auswertung)

\begin{tabular}{|c|c|c|c|}
\hline $\begin{array}{l}\text { Naturschutzfachli- } \\
\text { ches Ziel } \\
\text { (exemplarisch) }\end{array}$ & $\begin{array}{l}\text { Mögliche Maßnahmen zur } \\
\text { Zielerreichung }\end{array}$ & $\begin{array}{l}\text { Mögliche Konsequenzen für die Wald- } \\
\text { bewirtschaftung }\end{array}$ & $\begin{array}{l}\text { Mögliche finanzielle } \\
\text { Konsequenzen }\end{array}$ \\
\hline \multirow{2}{*}{$\begin{array}{l}\text { Erhalt/Erhöhung der } \\
\text { Anzahl an Biotop- und } \\
\text { Altbäumen }\end{array}$} & \multirow{2}{*}{$\begin{array}{l}\text { Belassen von Habitat- und } \\
\text { Altbäumen als Einzelbäume } \\
\text { oder in Gruppen }\end{array}$} & $\begin{array}{l}\text { Einzelstammweiser oder gruppenwei- } \\
\text { ser Nutzungsverzicht, Erhöhung der } \\
\text { Produktionszeit }\end{array}$ & Minderertrag \\
\hline & & $\begin{array}{l}\text { Erhöhte Arbeitssicherheitsmaßnah- } \\
\text { men, erhöhte Verkehrssicherungs- } \\
\text { pflichten, Mehrbedarf an Personalschu- } \\
\text { lungen, Forstschutzrisiko }\end{array}$ & Mehraufwand \\
\hline \multirow[b]{2}{*}{$\begin{array}{l}\text { Erhalt/Erhöhung des } \\
\text { Anteils lebensraum- } \\
\text { typischer Gehölzarten }\end{array}$} & \multirow{2}{*}{$\begin{array}{l}\text { Senkung des Anteils } \\
\text { und/oder Verbot der Ein- } \\
\text { bringung gesellschaftsfrem- } \\
\text { der Gehölzarten, } \\
\text { Einbringung von Misch- } \\
\text { baumarten, Waldumbau, } \\
\text { Belassung von Samenbäu- } \\
\text { men, Naturverjün- } \\
\text { gung/Wildlinge }\end{array}$} & Einschränkungen der Baumartenwahl & Minderertrag \\
\hline & & $\begin{array}{l}\text { Reduktion gesellschaftsfremder Baum- } \\
\text { arten }\end{array}$ & Mehraufwand \\
\hline \multirow{2}{*}{$\begin{array}{l}\text { Verbesserung der } \\
\text { Waldstruktur }\end{array}$} & \multirow{2}{*}{$\begin{array}{l}\text { Kleinflächige Nutzung, } \\
\text { Verlängerung der Produkti- } \\
\text { onszeit, lange Verjüngungs- } \\
\text { zeiträume, Flächenstillle- } \\
\text { gung, Umwandlung in Laub- } \\
\text { oder Mischwald, Verbot von } \\
\text { flächiger Endnutzung }\end{array}$} & $\begin{array}{l}\text { Nutzungsverzicht, Produktionsverzicht, } \\
\text { Absenkung von Eingriffsstärken, Ein- } \\
\text { schränkungen der Baumartenwahl }\end{array}$ & Minderertrag \\
\hline & & $\begin{array}{l}\text { Wenig rationelle Bewirtschaftungsme- } \\
\text { thoden, Verzicht auf hochmechanisier- } \\
\text { te Holzernte, Waldrandgestaltung, ggf. } \\
\text { erhöhter jagdlicher Aufwand }\end{array}$ & Mehraufwand \\
\hline
\end{tabular}

Von Relevanz sind für diese Berechnungen ausschließlich die durch naturschutzfachliche Maßnahmenplanungen von Veränderungen betroffenen betrieblichen Steuerungsgrößen. So kann der Bo- 
denwert, unter der Annahme, dass der Waldbesitzer den Wald vor Umsetzung der Naturschutzmaßnahmen nicht neu kauft, vernachlässigt werden. Unter die zu bewertenden Steuergrößen können erhöhte Kultur-, Läuterungs- und Holzerntekosten, Ertragsänderungen durch Baumartenwechsel oder Stilllegung von Teilflächen sowie Produktionszeitänderungen fallen. Ebenso können naturschutzmaßnahmenbezogene Verwaltungskosten auftreten. Hierbei könnte es sich um Kosten, die abhängig sind von der Größe und Naturalausstattung der jeweiligen betrieblichen Schutzgebietsfläche (variable Kosten), wie Monitoring oder die Organisation/Koordination von Verkehrssicherungsmaßnahmen bei hohen Altholzanteilen oder um fixe Kosten, wie z.B. die Einstellung einer zusätzlichen Verwaltungsfachkraft für die Umsetzung von naturschutzfachlichen Maßnahmen, handeln.

Eine besondere Herausforderung bei der Bewertung der Auswirkungen naturschutzfachlicher Maßnahmenplanungen sind die langen Wirkungszeiträume in der Forstwirtschaft. Aufgrund der "langen Lebenszyklen von Waldbäumen zwischen einer waldbaulichen Entscheidung, ihrer Realisierung und der möglichen Beobachtung und Begutachtung von allen Handlungsfolgen [liegen] viele Jahrzehnte bis hin zu Jahrhunderten [...] [ Daher ist eine] direkte Konfrontation eines heute tätigen Bewirtschafters mit allen Folgen seiner Entscheidung [...] zumeist nur auf Grundlage von [...] Modellüberlegungen möglich“ (OESTEN und ROEDER 2002:140). Um die Auswirkungen einiger naturschutzfachlicher Maßnahmenplanungen auch langfristig sichtbar zu machen, bedarf es eines geeigneten Modells. Mit diesem Modell sollten nicht nur die kurzfristige Änderung von Deckungsbeiträgen im Zeitverlauf, sondern auch die langfristige Ertragswertänderungen über bestimmte Zeiträume abbildbar sein. Letztere Werte sind insbesondere für die forstliche Praxis relevant. Für politische Entscheidungsträger dagegen sind nicht nur Ergebnisse auf Forstbetriebsebene, sondern oft auch auf höherer räumlicher Aggregationsebenen (z.B. die deutsche Natura 2000-Schutzgebietskulisse im Wald) wichtig. Mit dem Modell sollten folglich auch Bewertungen für Regionen, Bundesländer oder die gesamte Bundesrepublik durchführbar sein.

Als Grundlage für die Bewertungen naturschutzfachlicher Maßnahmenplanungen bedarf es zum einen Informationen zu allgemeinen Betriebsdaten und den Bewirtschaftungskonzepten betroffener Forstbetriebe. Zum anderen bedarf es für die Bewertungen operationalisierter Naturschutzmaßnahmenplanungen. Während betriebliche Daten beispielsweise durch die Zusammenarbeit mit Forstbetrieben relativ einfach verfügbar gemacht werden können, sind naturschutzfachliche Maßnahmenplanungen in ihrer Ausgestaltung jedoch bisher eher qualitativ und müssen für ökonomische Bewertungen erst operationalisiert werden (vgl. ROSENKRANZ et al. 2012).

\subsubsection{Bewertung der Auswirkungen naturschutzfachlicher Maßnahmen auf die Forstwirtschaft}

Für ein umfassendes Bild der Auswirkungen naturschutzfachlicher Maßnahmenplanungen müssten die durch die Umsetzung entstehenden Kosten sowie auch der monetäre und ökologische Nutzen der 
Maßnahmen bewertet werden. Der Einsatz von Produktionsfaktoren durch die Forstbetriebe müsste sowohl den kurz- und langfristigen Wirkungen für einzelne Naturschutzgüter als auch für die gesamte Biodiversität gegenübergestellt werden.

Eine Möglichkeit für die Nutzenbewertung sind Studien zur Zahlungsbereitschaft, wie z.B. zur Zahlungsbereitschaft für Natura 2000-Gebiete in Schottland (GIBSON et al. 2004) und Finnland (POUTA et al. 2000, LI et al. 2004). Studien solcher Art bewerten jedoch nicht den eigentlichen Nutzen der Maßnahmen für die Biodiversität, wie beispielsweise, ob eine Erhöhung des Laubaltholzanteils tatsächlich zu einem höheren Artenspektrum im Wald führt. Die langfristigen Wirkungen von Naturschutzmaßnahmen und ihre Wirksamkeit hinsichtlich der Zielsetzung, d.h. Schutz, Erhalt und Mehrung der Biodiversität, sind aber auch noch weitgehend unbekannt. Eine solche Nutzenbewertung von Naturschutzmaßnahmen im Rahmen dieser Studie wäre wünschenswert, jedoch aus forschungsökonomischen Gründen nicht durchführbar. Daher liegt ein Fokus der vorliegenden Arbeit auf den ökonomischen Auswirkungen naturschutzfachlicher Maßnahmen für Forstbetriebe. Hierbei wird analysiert, ob und in wie weit sich aus der Umsetzung der Maßnahmen ein monetärer Nutzen (z.B. erhöhte Deckungsbeiträge) oder Opportunitätskosten ${ }^{6}$ für Forstbetriebe ergeben. Die Ergebnisse könnten zum Beispiel als Basis für Kompensationszahlungen oder für politische Entscheidungen als Teil einer zukünftigen Kosten-Nutzen-Analyse verwendet werden. Für die Entwicklung der Methode im Rahmen dieser Arbeit ergeben sich aus Kapitel 1.4.1 folgende Notwendigkeiten:

- Vergleich einer Waldbewirtschaftung "mit" zu einer Waldbewirtschaftung "ohne" naturschutzfachliche Maßnahmenplanungen, ggf. Berücksichtigung verschiedener Referenzen

- Berücksichtigung der betriebsindividuellen naturalen Ausstattung und Steuergrößen der Waldbewirtschaftung

- Nur Berücksichtigung von Steuergrößen, die für die Bewertung naturschutzfachlicher Maßnahmen relevant sind

- Verschiedene naturschutzfachliche Maßnahmen sollten zusammen bewertet werden können

- Bewertung der Auswirkungen naturschutzfachlicher Maßnahmenplanungen auf Betriebe/Betriebsteile, nicht auf Einzelbestände

- Abbildungen von Veränderungen durch naturschutzfachlicher Maßnahmen im Zeitverlauf, kurz- mittel und langfristige Auswirkungen sollten abbildbar sein

Zur Bewertung von Opportunitätskosten für die Umsetzung naturschutzfachlicher Maßnahmen gibt es zwei Möglichkeiten (oder eine Kombination daraus): Die Bewertung der durch den Naturschutz gebundenen Produktionsfaktoren (Boden, Arbeit und Kapital), die anderweitig nicht mehr eingesetzt werden können oder die Bewertung der Produkte, auf welche durch die naturschutzfachliche Maß-

\footnotetext{
${ }^{6}$ Als Opportunitätskosten werden in Rahmen dieser Arbeit die Kosten bezeichnet, die in der Forstwirtschaft durch Verzicht auf Rohholzproduktion zugunsten naturschutzfachlicher Maßnahmen entstehen.
} 
nahmenplanung verzichtet werden muss (HAMPICKE 1991). Im Rahmen einer umfassenden Bewertung in dieser Arbeit sollen sowohl Produktionsfaktoren, hier im Sinne von Arbeits- und Verwaltungskosten, als auch der Verzicht auf Produkte, im Sinne von Holzerntemengen, bewertet werden.

Verzichte auf Holzerntemengen könnten beispielsweise mit Hilfe von Waldwachstumsmodellen wie Silva (PRETZSCH et al. 2002), Waldplaner (HANSEN 2012) oder BWINPro (DöBBeler et al. 2011) durchgeführt werden. Diese beinhalten jedoch keine wirtschaftlichen Komponenten und rechnen ausschließlich auf der Ebene von Beständen oder Hektaren. Sie sind daher für die Bewertungen im Rahmen dieser Arbeit nur teilweise geeignet.

Weitere Bewertungsmöglichkeiten bieten die klassischen Methoden der Waldwertermittlung oder ökonomischen Modelle, die auch teilweise auf die Waldwertermittlungsmethoden aufbauen. Bewertungsobjekte der Waldwertermittlung sind die Bodenbewertung, die Bestandsbewertung und die Betriebsbewertung. Gemäß OESTEN und ROEDER (2002) können dabei folgende Wertarten unterschieden werden:

- Sach- oder Substanzwert: Der Wert eines Gutes bemisst sich am Tageswert gleicher Güter, die auf dem Markt angeboten werden (z.B. Abtriebswert eines Bestandes).

- Kostenwert: Der Wert eines Gutes bemisst sich aus den innerbetrieblichen Kosten, die zu seiner Herstellung in der Vergangenheit benötigt wurden (z.B. Bestandeskostenwert).

- Ertrags- oder Erwartungswert: Der Wert eines Gutes bemisst sich aus dem Wert des zukünftigen Nutzens (z.B. Bestandeserwartungswert). Dabei wird der Ertragswert als Kapitalwert der zukünftigen, auf das Gut bezogenen Ein- und Auszahlungen bestimmt.

Die Eignung bestehender klassischer Bewertungsverfahren für die Erfordernisse im Rahmen dieser Arbeit soll im Folgenden diskutiert werden.

\section{Bodenbewertung}

Bei der Bodenbewertung steht der Waldboden als Produktionsgrundlage der Forstwirtschaft im Fokus der Bewertungen. Dabei beinhaltet der Begriff "Waldboden“ den bloßen Boden (ohne Bestockung) als „Substrat der nachhaltigen Forstwirtschaft“ (SAGL 1992:72). Die Bewertung des Waldbodens kann mit Hilfe des Bodenertragswerts (auch: Bodenerwartungswert), des Verkehrswerts und der Bodenrente erfolgen.

Die Berechnung des Bodenertragswerts geht zunächst von einem holzleeren Boden aus, der im Folgenden ewig als Wald bewirtschaftet wird (OESTEN und ROEDER 2002). Der Bodenertragswert umfasst alle Erträge, die auf Waldboden unter bestimmten Bodenverhältnissen während der Dauer der Produktionszeit abgeworfen werden, abzüglich der Aufwendungen für die Produktion (SPEIDEL 1967). Bei den Erträgen handelt es sich um den Abtriebswert zum Ende der Produktionszeit sowie um Erträge aus Vor- und Nebennutzungen, bei den Aufwendungen beispielsweise um Kultur- und Läuterungs-, 
Holzernte- und Verwaltungskosten. Die Aufwendungen und Erträge, die im Laufe des Bestandslebens zu unterschiedlichen Zeitpunkten anfallen, werden bei der Bodenertragsbewertung auf den Zeitpunkt der Produktionszeit bezogen. Hierbei wird unterstellt, dass stets die gleiche Baumart angebaut wird und die Erträge, Aufwendungen und Bodenleistungsfähigkeit konstant bleiben. Die heute gängige Formel zur Berechnung des Bodenertragswerts wurde von FAUSTMANN (1849) entwickelt (FAUSTMANN-Formel).

Mit dem Bodenverkehrswert wird der Preis bezeichnet, der sich für das Wirtschaftsgut Boden durch Ausgleich von Angebot und Nachfrage ergibt (SPEIDEL 1967). Ein gesicherter Verkehrswert für Waldböden ist jedoch schwer zu bestimmen, da es sich bei Forstbetrieben oder Waldbeständen nicht um homogene Güter und bei den gezahlten Preisen oft auch um Liebhaberwerte handelt (vgl. SPEIDEL 1967, SAGL 1992). Zur Bestimmung des Verkehrswerts können hilfsweise aktuelle Bodenpreise angrenzender vergleichbarer Flächen (Landwirtschaft, Offenland, Bauland) herangezogen werden. Der Waldbodenpreis ist dann abhängig von dem Preis der angrenzenden Grundstücke, die ihrerseits wiederum u.a. abhängig sind von der Infrastruktur, der Besiedelungsdichte und den Bonitäten. Alternativ können auch Richtpreise aus Waldbewertungsrichtlinien, abhängig von Holzart und Ertragsklasse, herangezogen werden (SPEIDEL 1967).

Die Bodenrente wird durch Verzinsung des Bodenwerts berechnet. Sie zeigt den Anteil des Produktionsfaktors Waldboden am Waldertrag auf. SAGL (1992) beschreibt die Bodenrente als den Wert, der sich jährlich aus den Erträgen des Waldbodens ergibt. Die Bodenrente kann dazu eingesetzt werden, eine Entschädigung bei dauerndem oder zeitweiligem Ertragsausfall, beispielsweise durch Stilllegungsflächen, zu bewerten (SAGL 1992).

Die hier vorgestellten Bodenbewertungsmethoden werden als weniger geeignet für die eigene Arbeit gewertet. Grundlage für die Berechnung des Bodenertragswerts ist der holzleere Boden, Bewertungsobjekt für die eigenen Fragestellungen jedoch bestockte Bestände. Veränderungen der Waldbewirtschaftung durch Integration naturschutzfachlicher Maßnahmen im Laufe des Bestandeslebens können nicht aufgezeigt und Baumartenwechsel nicht berechnet werden. Darüber hinaus werden hier idealisierte Normalwaldbestände mit festgelegten Produktionszeiten berechnet, was den natürlichen Gegebenheiten eines Forstbetriebes nicht entspricht.

Bei der Bewertung des Verkehrswerts stellt sich die Schwierigkeit, dass angrenzende vergleichbare Flächen nicht immer verfügbar sind und die Entwicklung des Bodenwertes in der Land- und Forstwirtschaft nicht immer gleich verläuft. Für die Bewertung der Auswirkungen naturschutzfachlicher Maßnahmen liegt die Schwierigkeit bei der Verwendung des Verkehrswertes auch darin, dass außer Boden und Bestockung keine weiteren Faktoren bewertet werden und Belastungen und Beschränkungen nur indirekt Teil der Bewertung sind. Außerdem ist die Waldumwandlung in andere Nut- 
zungsarten in Deutschland stark limitiert und reglementiert und die Bodenverkehrswerte anderer Nutzungsarten daher nur eingeschränkt übertragbar. Des Weiteren werden in der Praxis Waldgrundstücke meist zusammen mit dem aufstockenden Bestand verkauft. Die Aufteilung des Verkaufspreises in einen Bodenwert und einen Bestandeswert ist daher wenig aussagefähig und für die eigenen Zwecke nicht geeignet.

Auch die Berechnung der Bodenrente ist für die Bewertungen der naturalen und ökonomischen Auswirkungen durch naturschutzfachliche Maßnahmenplanungen nicht praktikabel: einerseits werden keine naturalen Änderungen bewertet und andererseits ist mit dieser komparativ-statischen Methode nur die Bewertung von Stilllegung oder der Vergleich von Erträgen unterschiedlicher Baumarten im Altersklassenwald, jedoch nicht die Bewertung eines dynamischen Baumartenwechsels und von ungleichaltrigen Beständen möglich.

\section{Bestandesbewertung}

Für die Bewertung eines Waldbestandes sind die Ermittlung des Abtriebswerts, des Bestandeskostenwerts und des Bestandeserwartungswerts übliche Methoden. Mit ihnen kann der Wert von Waldbeständen in unterschiedlichen Altersstufen bewertet werden. Sie können für Verkehrswertschätzungen und zur Ermittlung von Entschädigungs- und Schadensersatzzahlungen aber auch zur Betriebsbilanzierung und zur Bewertung von betrieblichem Vermögen und Erfolg eingesetzt werden. Der Bestandesbewertung liegt die Annahme eines aussetzenden Betriebes zugrunde. Sie bezieht sich, wie der Name schon sagt, auf Bestände, kann aber auch zur Bewertung von Dauerbetrieben eingesetzt werden. Bestandeswerte gehören definitorisch zu den Sach- oder Substanzwerten. Sie orientieren sich an Marktpreisen (SAGL 1992).

Der Bestandeskostenwert ist ein Wiederbeschaffungswert (SAGL 1992). Er wird zur Bewertung eines mehr oder weniger jungen Bestandes (SAGL (1992): bis 30 Jahre) eingesetzt. Bei der Berechnung des Bestandeskostenwerts wird unterstellt, dass der Wert eines Gutes mindestens so hoch ist, wie die Kosten, die zu seiner Herstellung aufgewendet wurden. Im Falle der Bestandesbewertung betrifft dies die Kultur-, Läuterungs-, und Verwaltungskosten. Diesen Kosten werden Erträge aus Durchforstungs- und Nebennutzungen bis zum Bewertungsstichtag gegengerechnet (SPEIDEL 1967, SAGL 1992). Kosten und Erträge fallen im Laufe des Bestandeslebens zu unterschiedlichen Zeitpunkten an. Gemäß SAGL (1992) können die Kosten dennoch so behandelt werden, als wären sie zum Zeitpunkt der Kulturbegründung angefallen. Er weist jedoch darauf hin, dass, streng finanzmathematisch, alle Kosten auf das Bewertungsalter prolongiert werden müsste.

Der Bestandeserwartungswert wird für die Bewertung von mittelalten Waldbeständen eingesetzt. Er ist ein Zukunftserfolgswert und berechnet sich aus dem Abtriebswert und den Erträgen eines Bestandes zwischen dem Alter $\mathrm{m}$ und der Umtriebszeit, abzüglich der Aufwendungen, die zwischen dem 
Alter $m$ und der Produktionszeit zu erwarten sind (SPEIDEL 1967, SAGL 1992). Die unterschiedlichen Beträge fallen auch beim Bestandeserwartungswert erwartungsgemäß zu unterschiedlichen Zeitpunkten an. Sie werden daher zunächst mit einem einheitlichen Zinsfuß auf den Zeitpunkt der Produktionszeit prolongiert und anschließend in Summe auf den Bewertungszeitpunkt diskontiert. Der Bestandserwartungswert entspricht zum Alter der Produktionszeit dem Abtriebswert des Bestandes. Der Abtriebswert kann auch als Zerschlagungswert bezeichnet werden. Er ist der zum Zeitpunkt des Bewertungsstichtages mit Marktpreisen bewertete, verkaufsfähige Holzvorrat eines Bestandes oder Bestandesteils abzüglich der Holzernte- und Vertriebskosten sowie dem Ernteverlust (SPEIDEL 1967, SAGL 1992). In der Praxis wird der Abtriebswert zur Bewertung von hiebsreifen Beständen eingesetzt. Mit dem Vergleich zweier Abtriebswerte eines Bestandes unter zwei möglichen Behandlungsvarianten lässt sich die Wertänderung des Bestandes durch Umsetzung verschiedener Waldbewirtschaftungszielen zum Ende des Bestandeslebens aufzeigen.

Mit den hier vorgestellten Bestandesbewertungsmethoden könnte der Wert eines Bestandes im Laufe seiner Produktionszeit mittels dreier Rechnungen bewertet werden. Die Werte zweier unterschiedlich behandelter Bestände könnten für junge und mittelalte sowie für hiebsreife Bestände mittels einer Vergleichsrechnung bestimmt werden. Durch die Aufteilung in drei Rechenabschnitte könnte aufgezeigt werden, in welchem Bestandesaltersabschnitt die größten Veränderungen zu erwarten sind. Um die Wirkung einzelner Maßnahmen im Laufe eines Bestandeswachstums aufzuzeigen, müssten jedoch mehrere Rechnungen zu unterschiedlichen Zeitpunkten des Bestandeslebens durchgeführt werden (z.B. je Fünfjahresperiode). Für die Bewertung von naturschutzfachlichen Maßnahmenplanungen würden alle relevanten Kostenarten berücksichtigt werden. Sortimentsmengenänderungen, z.B. durch Flächenreduktionen müssten jedoch separat berechnet werden. Da die Methoden nur für einzelne Reinbestände im Laufe einer Produktionszeit (und nicht für die Bewertung ganzer Forstbetriebe oder Betriebsteile) geeignet sind, keine ungleichaltrigen Bestände bewertet werden können und für die Bewertung der Auswirkungen naturschutzfachlicher Maßnahmenplanungen viele Einzelrechnungen nötig sind, wird der Einsatz dieser Methoden als ungeeignet für die eigene Arbeit gewertet.

\section{Betriebsbewertung}

Im Gegensatz zu den bisher vorgestellten Methoden beschäftigt sich die Betriebsbewertung nicht mit Teilwerten eines Betriebes, wie dem Boden oder Einzelbeständen, sondern mit dem Gesamtwert des Waldes. Hierfür gibt es prinzipiell zwei Verfahren: die Berechnung der Sachwerte (Sachwertmethode) und die Berechnung der Erträge (Ertragswertberechnung). Bei der Gesamtbewertung nach der Sachwertmethode wird der Sachwert eines Forstbetriebes als Summe der bereits vorgestellten Methoden 
Boden- und Bestandesbewertung berechnet (Zerschlagungswert). Auf sie soll daher im Folgenden nicht näher eingegangen werden.

„Ertragswerte entstehen durch die Kapitalisierung von endlichen oder ewigen Renten, die jährlich oder periodisch sein können“ (SAGL 1992:127). Für die Ermittlung eines Ertragswerts wird der Betriebserfolg, im Sinne von jährlich eingehenden Renten (z.B. erntekostenfreie Erträge aus End- und Vornutzungen) eines Gesamtbetriebs, abzüglich von Kultur- und Verwaltungskosten, für einen festgelegten Zeitraum kapitalisiert. Gemäß MöHRING und RüPING (2006) müssen für die Bewertung der Auswirkungen unterschiedlicher Waldbewirtschaftungsvarianten mit Hilfe der Ertragswertberechnung zunächst die naturalen Auswirkungen (z.B. Verlust von Erntemengen) im zeitlichen Verlauf für alle Waldbehandlungsvarianten bestimmt werden. Im nächsten Schritt müssen diese naturalen Auswirkungen monetär bewertet werden (z.B. anhand von Deckungsbeiträgen). Aus diesen unregelmäßig eingehenden Zahlungsströmen unterschiedlicher Waldbewirtschaftung wird der Bar- oder Kapitalwert berechnet. „Der unter Verwendung der Methoden der dynamischen Investitionsrechnung üblicherweise ermittelte Kapitalwert oder Barwert des „Zahlungsstrom-Saldos“ entspricht dann dem Ertragsverlust" (MÖHRING und RÜPING 2006:3). Auf Basis der Ertragswertberechnung kann eine Annuitätenberechnung erfolgen. Hierbei wird der „laufend wechselnde Zahlungsstrom der Holzeinschläge in einen „derivaten“, jährlich konstanten, aber ökonomisch äquivalenten Zahlungsstrom [...] überführt" (MÖHRING und RÜPING 2006:6).

Ein wichtiges Beispiel für die Ertragswertberechnung ist der Waldrentierungswert. Der Waldrentierungswert beschreibt den Ertragswert eines nachhaltigen Forstbetriebes. Im Gegensatz zur Bodenoder Bestandesbewertung werden keine Einzelflächen, sondern die Gesamtbetriebsfläche einschließlich aller Wirtschaftsgüter (Bestände, Boden, Wege, etc...) des jeweiligen Forstbetriebs bewertet (SPEIDEL 1967). Hierbei wird grundsätzlich von einem Normalwald ausgegangen. Es lassen sich jedoch auch Waldrentierungswerte in Betrieben ohne Normalwaldverhältnisse berechnen. Der Waldrentierungswert wird dann aus dem Reinertrag mehrerer aufeinanderfolgender Perioden berechnet. Der Reinertrag berechnet sich dabei aus der Summe aller erntekostenfreien Holzerlöse aus Vor- und Endnutzungen abzüglich der Kultur- und Verwaltungskosten. Für die Berechnung des Waldrentierungswerts wird angenommen, dass die Reinerträge innerhalb ihrer Perioden in jährlich gleicher Höhe anfallen (SPEIDEL 1967, Oesten und ROEDER 2002).

Aufgrund der Möglichkeit, einen ganzen Betrieb oder Betriebsteil zu bewerten, wird die Ertragswertmethode für die eigenen Fragestellungen grundsätzlich als geeignet angesehen. Die eigenen Bewertungen orientieren sich dabei grundsätzlich am Vorgehen von MöHRING und RüPING (2006). Neu ist hierbei die Anwendung der Methode für die Bewertungen der naturschutzfachlichen Maßnahmenplanungen auf Forstbetriebe oder Forstbetriebsteile und die gesamte Bundesrepublik. 


\section{Weitere Ansätze zur Bewertung naturschutzfachlicher Maßnahmenplanungen}

Die hier genannten Waldbewertungsmethoden wurden teilweise bereits zur Bewertung der Auswirkungen naturschutzfachlicher Maßnahmenplanungen eingesetzt. POSAVEC et al. (2011) berechneten einmalige Ausgleichzahlungen für Flächenstilllegungen in FFH-Gebieten mit Hilfe des Kapitalwerts von Waldbeständen. MöHRING und RÜPING (2006) entwickelten die „Annuitäten-Methode“ zur Bewertung von langfristigen Effekten z.B. durch Änderung von Produktionszeiten oder Baumartenwechsel. WAGNER und JÖNSSON (2001) berechneten dagegen durchschnittliche Deckungsbeitragsänderungen durch die Umsetzung von FFH-Maßnahmen. KÜPPERS und DIETER (2006) bewerteten Einkommensverluste auf Stilllegungsflächen auf Basis der Buchführungsergebnisse der Teilnehmerbetriebe des Testbetriebsnetzes Forst und der Waldgesamtrechnung. AICHHOLZ et al. (2012) bewerteten naturale und monetäre Auswirkungen der FFH-Richtlinie auf Basis der Daten der BWI 2002, Göttinger Annuitätentabellen und verschiedener Restriktionstypen aus FFH-Maßnahmenplanungen für wichtige Baumartengruppen. Keine der hier aufgeführten Studien berücksichtigen jedoch betriebsindividuelle Eingangsdaten oder führt Berechnungen auf Betriebsebene oder die gesamte deutsche Forstwirtschaft unter Berücksichtigung mehrerer naturschutzfachlicher Maßnahmen durch.

\subsubsection{Herausforderungen bei der Bewertung von Preis- und Mengenänderungen auf die Holzwirtschaft}

Bei der Bewertung der Wirkungen von Mengen- und damit Preisänderungen - zum Beispiel durch veränderten Holzeinschlag - auf die Holzwirtschaft ergeben sich vielfältige Herausforderungen.

Zunächst müssen die Auswirkungen für einzelne Wirtschaftszweige bewertet werden (z.B. welche Wertschöpfung und Beschäftigung wird in einer Branche auf Basis einer Einheit Rohholz als Vorleistung aktuell generiert?). Informationen hierzu bieten bspw. die Kostenstrukturstatistik (StBA F4 R4.3), die Material- und Wareneingangsstatistik (StBA F4 R4.2.4) oder die Produktionsstatistik (StBA F4 R4.1) des Statistischen Bundesamts. In den verfügbaren Datengrundlagen sind jedoch nur Werte (d.h. Mengen mal Preise) und keine durchschnittlichen Maßeinheiten abgebildet. Für eine umfassende Darstellung der Auswirkungen bedarf es jedoch nicht nur Kenntnissen der Preis- und Mengenänderungen für einzelne Wirtschaftszweige, sondern es müssen die Wirkungen auf ganze Wertschöpfungsketten bewertet werden. Für diese Bewertungen sind Kenntnisse zu den Wertschöpfungszusammenhängen holzbasierter Wertschöpfungsketten des Clusters Forst und Holz erforderlich. Die Komplexität der in- und ausländischen wirtschaftlichen Vernetzungen sowie die teilweise kaum erkennbaren Systemgrenzen stellen hierbei eine große Herausforderung dar.

Betriebe der ersten Holzverarbeitungsstufe sind aufgrund der hohen Rohstofftransportkosten überwiegend auf das lokale oder regionale Vorkommen von Holzvorräten angewiesen. Mit der Kenntnis der Effekte veränderten Rohholzaufkommens auf die lokalen und regionalen Beschaffungsmärkte für 
ganze Wertschöpfungsketten der holzverarbeitenden Industrie könnten die Auswirkungen konkurrierender waldflächen- und rohholzbasierter Nutzungsoptionen in Regionen abgeschätzt werden. Die hierfür benötigte Datengrundlage (z.B.: Waldfläche, Altersklassenverteilung, Baumartenanteile, Einschlag, interregionale Handelsbeziehungen) dürfte in Regionen jedoch unzureichend oder aufwendig zu erheben sein. Belastbare Wertschöpfungsanalysen auf regionaler Ebene sind daher bislang mit hohem Aufwand verbunden.

In den vergangenen Jahren stiegen die Anschaffungskosten für holzbasierte Rohstoffe, Halb- und Fertigwaren als Vorleistung in der holzbe- und -verarbeitenden Industrie an, während die Wertschöpfung teilweise sank (MANTAU 2012, SEINTSCH 2012). Vor diesem Hintergrund sind die Analyse des Einflusses einzelner Wertschöpfungseffekte (hier: Produktpreiseffekt, Vorleistungspreiseffekt, Wachstumseffekt und Struktureffekt), insbesondere aber auch der Zusammenhang zwischen holzbasierten Vorleistungen und den Wertschöpfungseffekten, in holzverarbeitenden Wirtschaftszweigen von Interesse. Für die Bewertung von Wertschöpfungseffekten bedarf es der Kenntnis von Preis- und Mengenänderungen des Outputs und Inputs, anhand derer Wachstumsfaktoren berechnet werden können. Hierfür kann auf Daten des Statistischen Bundesamtes zurückgegriffen werden. Eine Herausforderung stellt jedoch die geringe Differenzierung der einzelnen Statistiken, die teilweise Lückenhaftigkeit der Daten, die Datenverfügbarkeit (teilweise vierjährige Erscheinung), die unterschiedlichen Erfassungseinheiten sowie die Umstellung der Wirtschaftszweigklassifikationen im Betrachtungszeitraum dar. Weiterhin bedarf es der Kenntnis der Mengenänderungen des Inputs und Outputs in der Holzindustrie. Als Datenbasis stehen für die deutsche Holzindustrie die „Kostenstrukturerhebung im Verarbeitenden Gewerbe, Bergbau sowie in der Gewinnung von Steinen und Erden“ (StBA: F 4 R 4.3) und die "Material- und Wareneingangserhebung im Verarbeitenden Gewerbe sowie im Bergbau und in der Gewinnung von Steinen und Erden“ (StBA: F 4 R 4.2.4) zur Verfügung. Jedoch sind die Daten in beiden Statistiken nicht differenziert genug, um Aussagen über Wert und Menge von Vorleistungen treffen zu können.

\subsubsection{Bewertung der Auswirkungen von verändertem Holzeinschlag auf die Holzwirtschaft}

Für die Entwicklung der Methoden zur Abschätzung der Auswirkungen veränderten Holzeinschlags auf die Holzindustrie ergeben sich aus Kapitel 1.4.3 folgende Notwendigkeiten:

- Bestimmung der durchschnittlichen holzbasierten Wertschöpfung durch eine Einheit holzbasierter Rohstoffe, Halb- und Fertigwaren als Vorleistung in den einzelnen Wirtschaftszweigen und in Wertschöpfungsketten

- Bildung einer Datengrundlage zur Abschätzung der Auswirkungen veränderten Holzeinschlags auf Regionen im Rahmen der Wertschöpfungsanalyse

- Entwicklung und Anwendung einer Methode zur Analyse von Wertschöpfungseffekten 
- Bildung einer Datengrundlage zur Bestimmung der Ausgaben für Einzelpositionen von Vorleistungen in holzbasierten Wirtschaftszweigen

- Bestimmung von Vorleistungsmengen sowie deren Änderungen zwischen zwei Bewertungsjahren

Bisherige Untersuchungen zur holzbasierten Wertschöpfung wurden beispielsweise auf Basis von Clusterstudien, Input-Output-Tabellen oder anderen amtlichen Statistiken des Statistischen Bundesamtes durchgeführt. Im Rahmen der Clusterstudie Forst und Holz werden die Ressourcen und Kapazitäten der Branchen innerhalb der holzbasierten Wertschöpfungsketten untersucht. Hierzu sind bundesweite (z.B. SEINTSCH 2010, BeCher 2014) sowie auch landesweite Studien (z.B. SeEgmüLler 2005, RÜTHER et al. 2007, BORCHERT et al. 2008) verfügbar. In der Clusterstatistik werden Umsatz, Wertschöpfung und Beschäftigung für Branchen entlang der holzbasierten Wertschöpfungsketten dargestellt. Die Clusterstudien sagen jedoch nichts über die Verflechtung zwischen den Wirtschaftszweigen aus. Die Kennzahlen der einzelnen Branchen werden nur addiert und es werden keine Abhängigkeiten aufgezeigt. Außerdem sind sie nicht für einzelne Wirtschaftszweige differenziert: insbesondere in der zweiten Verarbeitungsstufe sind jeweils mehrere Wirtschaftszweige einer Branche zugeordnet. Die Kennzahlen können folglich nicht dem Input holzbasierter Vorleistungen im Sinne von Rohholzund Holzhalbwarenmengen zugeordnet werden. Darüber hinaus sind Wertschöpfung und Beschäftigung in der Holzwirtschaft nicht nur auf Bundes- oder Landesebene von Interesse. Als wichtige Arbeitgeber in ländlichen Regionen sind Kenntnisse über die Auswirkungen von verändertem Holzeinschlag auf einzelne Wirtschaftszweige und holzbasierte Wertschöpfungsketten insbesondere auch auf regionaler Ebene wichtig, bisher jedoch nicht verfügbar oder nur mit hohem Aufwand durchzuführen. Die Analyse von Clusterstatistiken wird daher für die eigenen Arbeiten als nicht geeignet betrachtet.

Input-Output-Analysen für den Holzsektor wurden unter anderem von MCGREGOR und MCNICOLL (2006), DieTER (2008) und BösCH et al. (2015a, b) durchgeführt. MCGREGOR und MCNicoll (2006) bewerteten mittels einer Input-Output-Analyse die Wirkung der Leistungen der Forstwirtschaft auf die Gesamtwirtschaft Großbritanniens. DIETER (2008) untersuchte die Wirkung einer zusätzlichen Einheit Rohholz auf die gesamtwirtschaftliche Wertschöpfung in Deutschland auf Basis einer Input-OutputAnalyse für das Jahr 2004. BösCH et al. (2015a) untersuchten im Rahmen einer physikalischen InputOutput-Analyse erstmalig den Holz- und Papierstrom im deutschen Wirtschaftssystem. BöscH et al. (2015b) untersuchten anhand von Input-Output-Tabellen interindustrielle Verbindungen des Clusters Forst und Holz in Deutschland. Für die Bewertung gesamtwirtschaftlicher Fragestellungen sind InputOutput-Analysen gut geeignet. Die Input-Output-Tabellen des Statistischen Bundesamtes weisen aber grundsätzlich nur eine sehr geringe Differenzierung auf (Produktionsbereiche: 02 „Forstwirtschaftliche Erzeugnisse und Dienstleistungen Forstwirtschaft“, 16 „Holz, Holz-, Flecht-, Korb- und 
Korkwaren (ohne Möbel)“, 17.1 „Holz- und Zellstoff, Papier, Karton und Pappe“ und 17.2 „Papier-, Karton- und Pappewaren“). Sie gelten für die gesamte Bundesrepublik und es lassen sich keine Bezüge zu Regionen herstellen. Für Fragestellungen der durchschnittlichen holzbasierten Wertschöpfung durch eine Einheit holzbasierten Rohstoffes sowie Wertschöpfungsänderungen auf regionaler Ebene müssten für jede Region eigene Input-Output-Tabellen erstellt werden. Die dafür benötigten Daten dürften nur schwierig und unter hohem Zeitaufwand zu erheben sein. Aus diesen Gründen wird die Input-Output-Analyse für die eigenen Analysen als ungeeignet erachtet.

SEINTSCH (2011) entwickelte eine Methode zur Bestimmung der Stellung der Holzrohstoffe in der Kostenstruktur der ersten Holzverarbeitungsstufe in Deutschland. Hierfür wurde die Kostenstrukturerhebung (StBA: F 4 R 4.3) mit der Material- und Wareneingangsstatistik (StBA: F 4 R 4.2.4) des Statistischen Bundesamtes verknüpft. Die Warengruppen der Material- und Wareneingangsstatistik wurden auf Basis weiterer Datenquellen sortimentsspezifisch nach Nadel- und Laubholz differenziert und daraus der gesamte Rohholzeinsatz der Wirtschaftszweige der ersten Verarbeitungsstufe berechnet. In Verbindung mit Daten aus der Kostenstrukturerhebung lassen sich Wertschöpfung und Beschäftigung je Einheit holzbasierter Vorleistungen für die erste Holzverarbeitungsstufe berechnen. Die Methode von SEINTSCH (2011) wird als geeignet für die eigenen Arbeiten angesehen. Sie wurde im Rahmen der eigenen Studie aktualisiert und zur Anwendung auf die zweite Holzverarbeitungsstufe weiterentwickelt.

Weiterhin ist eine Methode zur Analyse von Wertschöpfungseffekten zu entwickeln. Mit der Methode sollte geprüft werden können, ob Wertschöpfungsänderungen zwischen zwei Jahren durch Änderungen von Produktpreisen, Vorleistungspreisen, Outputmengen oder durch Strukturänderungen (Outsourcing/Offshoring) entstehen. Anhand der Methode sollte nach Möglichkeit auch der Zusammenhang zwischen holzbasierten Vorleistungen und den Wertschöpfungseffekten holzverarbeitender Wirtschaftszweige geprüft werden können. Im Rahmen der eigenen Arbeiten wurde hierfür eine Dekompositionsanalyse gewählt. Dekompositionsanalysen wurden bisher für unterschiedlichste Fragestellungen eingesetzt, z.B. um Änderungen einzelner Elemente, wie beispielsweise Wirtschaftswachstum, Wettbewerbsfähigkeit oder Beschäftigung, im Zeitverlauf zu untersuchen. So bewerteten KLEIN et al. (2009) mittels einer Shift-Share-Analyse regionale Beschäftigungsverhältnisse in der holzverarbeitenden Industrie in Deutschland, getrennt nach nationalen, sektoralen und regionalen Trends. IVANOV und WEBSTER (2010) untersuchten das bulgarische Wirtschaftswachstum mittels Dekomposition von Produktionsfaktoren, Ausgabenarten und dem Einfluss einzelner Wirtschaftszweige auf die Gesamtwirtschaft. DIETER und ENGLERT $(2007,2009)$ analysierten die Wettbewerbsfähigkeit der Holzindustrie in Deutschland, d.h. das Wachstum des Exports von Rohholz und Holzprodukten, anhand der Constant-Market-Share-Analyse nach MILANA (1988). Wertschöpfungsänderungen in der Holzindustrie wurden anhand der Dekompositionsmethode bislang nicht untersucht. 
Für die Berechnung von Wertschöpfungseffekten werden die Preise und Mengen der Inputs und Outputs sowie deren Veränderungen zwischen zwei Bezugsjahren benötigt. Um fundierte Aussagen über den Einfluss holzbasierter Vorleistungen auf die Wertschöpfungseffekte treffen zu können, ist die Kenntnis von Preisen und Mengen der einzelnen Vorleistungspositionen nötig. Hierfür kann die oben beschriebene Methode von SEINTSCH (2011) zur Verknüpfung der Material- und Wareneingangsstatistik mit der Kostenstrukturerhebung des Statistischen Bundesamtes herangezogen werden. 


\section{Einordnung der eigenen Ergebnisse in den fachlichen Kontext}

Im Folgenden werden die im Rahmen der eigenen Studie veröffentlichten Artikel vorgestellt und in den thematischen Kontext dieser Arbeit eingeordnet. Die ersten beiden Artikel beschäftigen sich mit der Fragestellung: „Welche Auswirkungen haben aktuelle naturschutzfachliche Maßnahmenplanungen auf die Forstwirtschaft?", die letzten drei Artikel mit der Fragestellung „Welche Auswirkungen hat der veränderte inländische Holzeinschlag auf die Holzindustrie?“.

Im Zuge der Umsetzung von Natura 2000 wurden im letzten Jahrzehnt große Waldflächen als FFHGebiete ausgewiesen. Die Erhaltungs- und Entwicklungsmaßnahmen für die FFH-Gebiete sind größtenteils festgelegt und derzeit in der Umsetzungsphase. Bislang fehlte jedoch ein geeignetes Instrument, um die kurz-, mittel und langfristigen Auswirkungen der Maßnahmen auf Forstbetriebe zu bewerten. Durch die Entwicklung, Eignungsprüfung und Anwendung einer Methode zur Bewertung der naturalen und wirtschaftlichen Auswirkungen von FFH-Maßnahmenplanungen auf Forstbetriebe wird diese Lücke in der Studie von RoSENKRANZ et al. (2014): „Income losses due to the implementation of the Habitats Directive in forests - Conclusions from a case study in Germany" geschlossen.

Eine weitere aktuelle, naturschutzfachliche Entwicklung ist die Forderung nach Segregation von Waldfunktionen auf Teilflächen, zur Anhebung des Waldnaturschutzniveaus in Deutschland. Bisher liegen noch keine Informationen für eine Politikfolgenabschätzung der Umsetzung dieser Forderungen vor. In der Studie von ROSENKRANZ und SEINTSCH (2015): „Opportunitätskostenanalyse zur Implementierung des naturschutzorientierten Waldbehandlungskonzepts „Neue Multifunktionalität““, werden Bewertungen zu den naturalen und ökonomischen Auswirkungen einer Segregation von Waldfunktionen auf Bundesebene durchgeführt. Die Ergebnisse können von politischen Entscheidungsträgern als Informationsgrundlagen verwendet werden.

Es ist anzunehmen, dass sich durch Umsetzung naturschutzfachlicher Maßnahmenplanungen der inländische Holzeinschlag reduzieren wird. Dies dürfte sich auf die Wertschöpfung und Beschäftigung der deutschen Holzindustrie auswirken. Bisher liegen nur wenige Studien zu den Auswirkungen von verändertem Holzaufkommen auf die Holzwirtschaft vor. Im Rahmen der Studie von ROSENKRANZ und SEINTSCH (2014): „Abschätzung der holzbasierten Wertschöpfung und Beschäftigung in stofflichen Wertschöpfungsketten“ wurde erstmalig ein Verfahren zur Bestimmung der Bruttowertschöpfung und Beschäftigung der holzverarbeitenden Wirtschaftszweige auf Basis einer Einheit holzbasierter Vorleistungen entwickelt. In der Studie von SEINTSCH und ROSENKRANZ (2015) „Wertschöpfungsanalyse von Waldflächen und Rohholz" wurde dieses Verfahren, sowie die im oberen Abschnitt genannte Methode zur Bewertung der Auswirkungen naturschutzfachlicher Maßnahmenplanungen, angewendet, um die Auswirkungen von verändertem Holzaufkommen in zwei Fallbeispielsregionen zu bewerten. So wurde ein einfaches Abschätzverfahren für die Wirkung von verändertem Rohholzaufkom- 
men auf holzbasierte Wertschöpfungsketten für Regionen entwickelt, für welches lediglich wenig Kenntnis zur Regionalwirtschaft (z.B. auf Basis der Umsatzsteuerstatistik und Forsteinrichtungsdaten) erforderlich ist.

Im Rahmen der Studie ROSENKRANZ et al. (2015): „Decomposition analysis of changes in value added. A case study of the sawmilling and wood processing industry in Germany" wurde schlussendlich ein Verfahren zur Bewertung von Wertschöpfungseffekten in der Holzindustrie entwickelt und erfolgreich angewendet. Mit diesem Verfahren können Wertschöpfungseffekte isoliert und ihr Einfluss auf die gesamte Bruttowertschöpfung von Wirtschaftszweigen analysiert werden. Zusätzlich ermöglicht diese Methode die Analyse des Zusammenhangs von holzbasierten Vorleistungen und Wachstum in der Holzindustrie.

\subsection{Erster Artikel: „Income losses due to the implementation of the Habitats Directive in forests - Conclusions from a case study in Germany"}

Dieser Artikel wurde von Lydia Rosenkranz, Dr. Björn Seintsch, Dr. Bernd Wippel und Prof. Dr. Matthias Dieter geschrieben und im Jahr 2014 in der Fachzeitschrift „Forest Policy and Economics" nach einem double blind peer-review veröffentlicht. Das Bewertungskonzept wurde von den Autoren gemeinsam entwickelt und auf Plausibilität geprüft. Lydia Rosenkranz und Dr. Bernd Wippel konkretisierten und operationalisierten die FFH-Maßnahmenplanungen gemeinsam mit den Fallbeispielsforstbetrieben, übersetzten diese in Modellsteuergrößen und führten die Berechnungen separat, jeweils für einen Teil der Forstbetriebe durch. Lydia Rosenkranz schrieb den Artikel. Prof. Dr. Matthias Dieter trug wesentlich zu den Schlussfolgerungen bei. Dr. Björn Seintsch war Co-Autor.

\subsubsection{Zusammenfassung}

Ziel der Studie war es, die naturalen und ökonomischen Auswirkungen von FFH-Maßnahmenplanungen auf Fallbeispielsforstbetriebe im laufenden FFH-Umsetzungsprozess zu ermitteln. Hierbei wurde methodisch ein wesentlicher Beitrag zur Weiterentwicklung und Plausibilisierung eines Simulationsmodells geleistet. Als Grundlage für die Bewertungen wurden FFH-Managementpläne sowie Betriebsdaten von 21 privaten und öffentlichen Fallbeispielsforstbetrieben in sechs Bundesländern herangezogen. Die Berechnungen wurden für die betrieblichen Buchenwald-Lebensraumtypflächen (9110 Hainsimsen- und 9130 Waldmeister-Buchenwald) durchgeführt. Als naturale und ökonomische Auswirkungen wurden nur die FFH-Maßnahmenplanungen bewertet, welche nicht im Einklang mit den betriebsindividuellen Bewirtschaftungszielen standen.

Als Grundlage für die Berechnungen wurde das ertragstafelbasierte forstbetriebliche Simulationsmodell von StRUGHOLTZ (2010) verwendet. Diese wurde an der Universität Göttingen im Rahmen einer Masterarbeit entwickelt und bisher nur auf einen Probebetrieb angewendet. Mit dem Modell können 
auf Basis forstbetriebsindividueller Eingangsdaten und Waldbauziele für vier Holzartengruppen die zukünftigen Holzeinschlagsmengen sowie waldbauliche Deckungsbeiträge (holzerntekostenfreie Erlöse abzüglich Kultur- und Läuterungskosten), Ertragswerte und Annuitäten im Verlauf eines 200jährigen Simulationszeitraums berechnet werden. Im Rahmen der Studie wurde das Modell intensiv anhand eines Normalwaldbetriebes geprüft und weiterentwickelt und in STRUGHOLTZ-ENGLERT-Modell umbenannt. So wurde das Modell z.B. um die Holzartengruppe Douglasie sowie um betriebsindividuelle Endnutzungszeiträume und Einschlagsintensitäten erweitert. Eine Besonderheit des Modells ist die Möglichkeit, mehrere naturschutzfachliche Maßnahmen gleichzeitig zu berechnen.

Für die Bewertungen wurden zwei Referenzen ohne die Umsetzung von FFH-Maßnahmenplanungen zugrunde gelegt. Die Referenz „Status quo“ spiegelte eine Fortführung der derzeitigen Waldbewirtschaftung des jeweiligen Forstbetriebs wider. Grund für die Bewertungen mit dieser Referenz ist das in politischen Diskussionen oft angeführte Argument, dass bei einer Umsetzung von FFH bei einer Fortführung des Status quo keine Belastungen für den Forstbetrieb entstehen würden. Mit der Referenz „Betriebsziel“ wurden zukünftige waldbauliche Zielsetzungen, ohne die Beachtung von naturschutzfachlichen Auflagen, in den Betrieben umgesetzt. Die Referenzen „Status quo“ und „Betriebsziel“ können, je nach Eigentümerzielsetzung, auch identisch sein. Nur die FFH-Maßnahmen, die von den Betrieben als Abweichung ihrer üblichen Bewirtschaftungsweisen empfunden wurden, wurden im Szenario „FFH-Regime" bewertet. Naturale und wirtschaftliche Auswirkungen konnten auf Basis des Saldos des Szenarios „FFH-Regime“ mit den beiden Referenzen bewertet werden.

Die FFH-Maßnahmen, die von den Betrieben als Abweichung ihrer üblichen Waldbewirtschaftungsweise empfunden wurden waren (1) die punktuelle Stilllegung durch dauerhafte Ausweisung von Habitatbäumen und Altholzinseln (2) die Erhaltung und Vermehrung lebensraumtypischer (Laub)Baumarten (d.h. Einschränkungen bei der Baumartenwahl, z.B. ertragsstarke Baumarten) sowie (3) der Erhalt eines hohen Anteils an Bäumen in der Reifephase. Für alle Forstbetriebe ergaben sich im 200jährigen Simulationszeitraum überwiegend Verluste an Holzerntemengen, Deckungsbeiträgen und Ertragswerten. Im Vergleich zwischen der Referenz „Betriebsziel“ und einer Umsetzung der FFHMaßnahmenplanungen („FFH-Regime“) ergaben sich, im Durchschnitt über alle Forstbetriebe, Verluste an Holzerntemengen von 0,66 $\mathrm{m}^{3} / \mathrm{ha} / \mathrm{a}$, Verluste an walbaulichem Deckungsbeitrag einschließlich erhöhter Verwaltungskosten, von $40 € /$ ha/Jahr und Ertragswertverluste von $2.496 € /$ ha. Die Verluste an Holzerntemengen und waldbaulichen Deckungsbeiträgen sind im Verlauf des Bewertungszeitraums starken Schwankungen unterlegen. Diese sind vor allem auf die Altersklassenausstattung der Betriebe sowie die Restriktion für den Erhalt der lebensraumtypischen Baumarten, d.h. kein weiteres Nadelholz zu pflanzen, zurückzuführen. Erhöhungen der Holzerntemengen im Laufe der Simulationsperiode können sich für die Betriebe über kurze Zeiträume aus aufgeschobenen Nutzungsmengen aufgrund von Produktionszeitverlängerungen ergeben. Sie können den Verlust durch FFH- 
Maßnahmen im 200jährigen Simulationszeitraum jedoch nicht ausgleichen. Zwischen den einzelnen Fallbeispielsforstbetrieben können sich, in Abhängigkeit von betriebsindividuellen Zielsetzungen, große Unterschiede in den Verluste ergeben.

\subsubsection{Kritische Diskussion und Einordnung in den thematischen Rahmen}

Mit dem StRUGHOLTZ-ENGLERT-Simulationsmodell wurden erstmalig die Auswirkungen mehrerer naturschutzfachlicher Maßnahmen auf Forstbetriebe im Zeitverlauf berechnet. Die Ergebnisse können als Hilfestellungen für Entscheidungsträger, z.B. bei der Entwicklung von Konzepten für Kompensationszahlungen herangezogen werden.

Für die Bewertungen wurde das Simulationsmodell von STRUGHOLTZ (2010) weiterentwickelt und angewendet. Das Modell basiert u.a. auf betriebsindividuellen Eingangsdaten und Ertragstafeln und bietet die Möglichkeit, mehrere forstatypische, naturschutzfachliche Maßnahmen gleichzeitig zu berechnen. Das Modell ermöglicht, im Gegensatz zu komparativ-statischen Bewertungsansätzen wie z.B. der FAUSTMANN-Formel, eine dynamische Analyse der Auswirkungen naturschutzfachlicher Maßnahmenplanungen. Hiermit ist es für die gegebene Zielsetzung besonders geeignet. Die Ergebnisse des 200-jährigen Simulationszeitraums unterliegen zwar grundsätzlich hohen Unsicherheiten. Für strategische Entscheidungen (z.B. über Baumartenwechsel) ist jedoch die Bewertung langer Produktionszeiträume erforderlich. Kritisch anzumerken ist, dass das Modell für den gesamten 200-jährigen Simulationszeitraum gleichbleibende, statische Holzerntekosten und Holzerlöse unterstellt. Ebenso können vom Altersklassenwald abweichende Bestände (z.B. Plenterwälder) nicht bewertet werden. Weiterhin können bei der Berechnung keine standörtlichen und klimatischen Veränderungen berücksichtigt werden. Hier herrscht weiterer Modellentwicklungsbedarf.

Im Jahr 2012 wurden in der Bundesrepublik im Staatswald über alle Holzartengruppen durchschnittlich 6,2 $\mathrm{m}^{3} /$ ha Holzbodenfläche, im Körperschafts- und Privatwald je $6,5 \mathrm{~m}^{3} /$ ha Holzbodenfläche eingeschlagen (ERMISCH et al. 2014). Hierbei handelt es sich um Durchschnittswerte über alle Baumarten, einschließlich des „Brotbaums“ Fichte. Zum Vergleich: Die Verkaufsmenge der Buche wurde von ERMISCH et al. (2013) im Mittel der Jahre 2003 und 2011 mit 3,6 m³/ha angegeben. Der im Vergleich zur Referenz „Betriebsziel“ berechnete durchschnittliche jährliche Verlust von 0,66 Efm/ha/Jahr entspricht folglich rund 9-10 \% der von ERMISCH et al. (2013) ermittelten durchschnittlichen Holzeinschlagsmenge über alle Baumarten und $18 \%$ der Verkaufsmenge der Buche. Hochgerechnet auf die Gesamtfläche der bewerteten Buchenwald-Lebensraumtypen von 0,55 Mio. ha in Deutschland ergibt sich ein jährlicher Verlust von rund 363.000 Efm/Jahr. Bei einer Nutzung von rund 76 Mio. Efm pro Jahr auf Bundesebene ist dies eine eher marginale Reduktion. Möglicherweise könnten sich jedoch Engpässe bei der regionalen Holzindustrie ergeben, insbesondere wenn in der jeweiligen Region weitere Schutzgebiete (z.B. Nationalpark) ausgewiesen werden. 
Der Reinertrag II der Produktbereiche 1-5 der laubholzdominierten Forstbetriebe ${ }^{7}$ des Testbetriebsnetzes Forst lag im Jahr 2013 im Körperschaftswald bei $83 €$ /ha Holzbodenfläche (HB) und im Privatwald bei 99 €/ha HB (BMEL 2015a, b). Der errechnete Verlust an waldbaulichem Deckungsbeitrag durch die Umsetzung von FFH-Maßnahmen im Vergleich zur Referenz „Betriebsziel“ von $40 € / \mathrm{ha} / \mathrm{Jahr}$ könnte den Reinertrag je Hektar Buchenlebensraumtypflächen der Betriebe erheblich senken. Im Falle des Staatswaldes, der erst ab dem Jahr 2010 überhaupt ein kontinuierlich positives Gesamtbetriebsergebnis verzeichnen konnte (ERMISCH et al 2014), würde der Reinertrag auf diesen Flächen ins Negative sinken. Setzt sich der derzeitige Trend der Steigerung der Holzpreise weiter fort, so erhöhen sich auch die Opportunitätskosten für die Umsetzung von naturschutzfachlichen Maßnahmen. Je nach Anteil der Buchenlebensraumtypflächen sind die Auswirkungen auf einzelne Betriebe jedoch sehr unterschiedlich.

Nicht alle geplanten FFH-Maßnahmen wurden von den Fallbeispielsforstbetrieben zum Zeitpunkt der Datenaufnahme als Einschränkung ihrer waldbaulichen Bewirtschaftungspraktiken empfunden. Die für Forstbetriebe besonders belastenden Nutzungseinschränkungen decken sich jedoch mit den von naturschutzfachlicher Seite häufig geforderten Maßnahmen "Stilllegung“ sowie „Erhöhung des Altholz- und Laubholzanteils". Durch die Studie konnte aufgezeigt werden, dass die Umsetzung dieser Maßnahmen sowohl kurz- als auch langfristig zu Holzernteverlusten und in der Folge zu verminderten Erträgen für Forstbetriebe führt.

Die Umsetzung der FFH-Maßnahmenplanungen befindet sich derzeit im Anfangsstadium und unterliegt daher noch hohen Unsicherheiten. Bisher sind Maßnahmenplanungen in FFH-Managementplänen noch wenig operationalisiert und lassen viel Spielraum für Interpretationen. Es wäre daher denkbar, dass zukünftig im Rahmen der Umsetzung im laufenden Betrieb und ggf. weiterer Konkretisierungen der Maßnahmen seitens des Naturschutzes noch weitere oder andere FFH-Maßnahmen von den Forstbetrieben als Belastung empfunden werden. Auch ist denkbar, dass die Ergebnisse durch die bewerteten 21 Forstbetriebe beeinflusst wurden. So könnten gerade die teilnehmenden Betriebe die FFH-Umsetzung als große Belastung empfinden während andere, nicht teilnehmende Betriebe, weniger Probleme mit der Umsetzung haben.

Ebenfalls könnten sich durch klimatische oder standörtliche Veränderungen sowie Änderungen der gesellschaftlichen oder betriebsindividuellen Ziele Annahmen und Ergebnisse im Laufe der 200jährigen Simulationsperiode ändern. Beispielsweise wäre denkbar, dass zur Stabilisierung der Wälder im Klimawandel von der Gesellschaft zukünftig wieder eine Erhöhung der Nadelholzanteile (in Form von Mischwäldern) gefordert würde oder dass neue Studien zu einer besseren Erkenntnis über die Menge benötigter Habitatbäume im Wald führten. Durch die Anpassung von Nadelholzanteilen und

\footnotetext{
${ }^{7}$ Hierbei handelt es sich um Forstbetriebe mit einem Laubholzanteil von über $50 \%$ der Wirtschaftsfläche.
} 
Habitatbaumzahlen würden sich dann entsprechend auch die Deckungsbeitragsverluste durch Umsetzung der Maßnahmen erhöhen oder senken.

\subsection{Zweiter Artikel: „Opportunitätskostenanalyse zur Implementierung des naturschutz- orientierten Waldbehandlungskonzepts „Neue Multifunktionalität"““}

Dieser Artikel wurde von Lydia Rosenkranz und Dr. Björn Seintsch geschrieben und im November 2015 bei der peer-reviewten Fachzeitschrift "Landbauforschung" eingereicht. Das Bewertungskonzept wurde von beiden Autoren gemeinsam entwickelt. Lydia Rosenkranz operationalisierte die naturschutzfachlichen Maßnahmen, führte die Berechnungen durch und schrieb den Artikel. Dr. Björn Seintsch war Co-Autor.

\subsubsection{Zusammenfassung}

In den letzten Jahren wurden Forderungen nach einer Segregation von Waldfunktionen auf Teilflächen laut. Die Forderungen sind jedoch größtenteils nicht operationalisiert. Auch sind bisher noch keine Bewertungen der naturalen und wirtschaftlichen Auswirkungen verfügbar. Vor diesem Hintergrund ist das Ziel dieser Studie, die bisher weitgehend allgemein gehaltenen Forderungen zu konkretisieren und die Opportunitätskosten einer Umsetzung der Segregation zum Zwecke des Naturschutzes auf die deutsche Forstwirtschaft zu ermitteln.

Im Rahmen dieser Studie wurde das STRUGHOLTZ-ENGLERT Simulationsmodell zur Bewertung der naturalen und ökonomischen Auswirkungen der Segregation von Waldfunktionen auf die deutsche Forstwirtschaft angepasst und die Auswirkungen mehrerer naturschutzfachlicher Maßnahmen, unter Berücksichtigung unterschiedlicher Bewirtschaftungsintensitäten, für den gesamten deutschen Wald berechnet. Hierfür wurden zwei Waldbehandlungsvarianten miteinander verglichen. Die Waldbehandlungsvariante "Status quo" beinhaltet die heutige Waldbewirtschaftung einschließlich des aktuellen Waldnaturschutzniveaus. Die Waldbehandlungsvariante "Neue Multifunktionalität" beinhaltet eine Operationalisierung der naturschutzfachlichen Ansprüche an den Wald. Für beide Bewirtschaftungsvarianten wurde eine Segregation der Wälder in Teilflächen mit unterschiedlicher Bewirtschaftungsintensität unterstellt. Sie orientiert sich an der Vorstellung einer neuen Multifunktionalität (HöLTERMANN 2013). Danach werden folgende Intensitätsstufen unterschieden: (1) Wirtschaftswald mit naturschutzfachlichen Mindeststandards, (2) Wald mit Naturschutzvorrangfunktion und (3) Wald ohne forstliche Bewirtschaftung. Mittels Literaturrecherche wurden die naturschutzfachlichen Maßnahmen bestimmt, deren Umsetzung starke Einschränkungen für Waldbesitzer erwarten lassen. Hierzu zählen der Erhalt bzw. die Erhöhung der lebensraumtypischen Artenzusammensetzung, der Habitatbaumanzahlen, des Totholz- und des Altholzanteils. Weiterhin wurden auf Basis von Literaturrecherchen und eigenen Annahmen Steuergrößen für den Status quo sowie für die Operationalisierung der Maßnahmen festgelegt. Für die Berechnungen wurden neben dem STRUGHOLTZ-ENGLERT- 
Simulationsmodell auch der Totholzkalkulator der Nordwestdeutschen Forstlichen Versuchsanstalt (MEYER et al. 2009) eingesetzt.

Für die Studie wurde der Ansatz der Opportunitätskostenanalyse gewählt. Im Ergebnis entstehen durch eine Umsetzung der Waldbehandlungsvariante "Neue Multifunktionalität" Verluste an Holzeinschlag von rund 11,2 Mio. Efm/Jahr und an waldbaulichem Deckungsbeitrag von rund 1 Mrd. $€ / J a h r$ für die deutsche Forstwirtschaft. Die Ertragswertdifferenzen der beiden Waldbehandlungsvarianten betragen über einen Zeitraum von 20 Jahren rund $21 \mathrm{Mrd}$ € und über den gesamten 200jährigen Simulationszeitraum rund 60 Mrd. €.

\subsubsection{Kritische Diskussion und Einordnung in den thematischen Rahmen}

Für die Berechnungen im Rahmen dieser Studie wurde das STRUGHOLTZ-ENGLERT-Simulationsmodell erstmalig auf Bundesebene, auf Datenbasis der BWI 2012, angewendet („Deutschlandbetrieb“). Durch die Berücksichtigung von Mehraufwand und Mindertrag durch mehrere naturschutzfachliche Maßnahmen werden naturale und ökonomische Auswirkungen durch die Umsetzung eines höheren Waldnaturschutzniveaus auf die gesamte deutsche Forstwirtschaft abschätzbar. Die Ergebnisse können als Folgenabschätzungen für politische Entscheidungen herangezogen werden.

Aus den Ergebnissen werden hohe Belastungen für die deutsche Forstwirtschaft erkennbar. Bei einem durchschnittlichen jährlichen Holzeinschlag von 76 Mio. Efm (BWI 2012) entspricht ein Verlust von 11,2 Mio. Efm rund $15 \%$ des Gesamteinschlags. Bei einer Umsetzung der Waldbehandlungsvariante „Neue Multifunktionalität“ würde sich insbesondere das forst- und holzwirtschaftlich bedeutsame Nadelholzaufkommen im 200-jährigen Mittel im Vergleich zu einer Bewirtschaftung nach dem „Status quo" um $32 \%$ reduzieren.

Ein bedeutender Treiber der Reduktion des Gesamteinschlags ist der geforderte Waldumbau hin zu einem höheren Laubholzanteil. Im Laufe der 200-jährigen Simulationsperiode erhöht sich kontinuierlich der Laubholzeinschlag während der Nadelholzeinschlag stark absinkt. Die erhöhte Laubholzmenge kann die fehlende Nadelholzmenge in Zukunft jedoch nicht vollständig kompensieren. Hinzu kommt, dass in der Bundesrepublik derzeit größtenteils mittelstarkes Nadelholz verarbeitet wird, während Laubholz hauptsächlich der energetischen Nutzung zugeführt wird. Die Erhöhung der Produktion von (starkem) Laubholz zielt rein aus Sicht der Holzverwendung an den aktuellen Marktbedürfnissen vorbei. Für den 200-jähringen Simulationszeitraum ist jedoch denkbar, dass neue Produkte aus Laubholz zu neuer Nachfrage führen.

Im Jahr 2012 erzielte die deutsche Forstwirtschaft, u.a. durch hohe Einschläge und Holzpreise, einen Produktionswert zu Herstellungspreisen in Höhe von 4,8 Mrd. € - zwei Drittel davon aus der Erzeugung von Nadelrohholz - sowie einen Nettounternehmensgewinn von 1,34 Mrd. € (SEINTSCH und RoSENKRANZ 2014). Ein durchschnittlicher waldbaulicher Deckungsbeitragsverlust von rund $1 \mathrm{Mrd}$. $€ / J a h r$ 
durch die Anhebung des Waldnaturschutzniveaus würde den Nettounternehmensgewinn erheblich schmälern.

Dem Verlust an Holzeinschlagsmengen durch naturschutzfachliche Maßnahmenplanungen steht der steigende Holzbedarf entgegen. Können die fehlenden Holzmengen nicht aus dem Import oder aus anderen Quellen (z.B. Landschaftspflegeholz) gedeckt werden, so sind weitere Holzpreisanstiege und Marktanpassungen (z.B. Substitution von Holz durch andere ggf. weniger klimafreundliche Rohstoffe) zu erwarten. Dies könnte negative Folgen für die Wertschöpfung und die Beschäftigung in der Holzindustrie haben.

Kritisch ist anzumerken, dass die Studie größtenteils auf Annahmen basiert. Ob und in welchem Maß das Waldnaturschutzniveau schlussendlich nach den Forderungen von HöLTERMANN (2013) angehoben werden soll, ist bisher nicht festgelegt. Bislang werden naturschutzfachliche Maßnahmen hauptsächlich im öffentlichen Wald umgesetzt. Ob eine Umsetzung im Privatwald in derselben Intensität möglich ist, ist fraglich. Denkbar wäre, dass zur Befriedigung der steigenden Nachfrage Einschlagseinbußen im öffentlichen Wald durch erhöhten Einschlag im Privatwald ausgeglichen werden müssten. Hier zeigt jedoch die BWI 2012, dass, ausgehend vom aktuellen Einschlag, die Potenziale für eine weitere Einschlagsteigerungen im Privatwald gering sind.

Neben den getroffenen Annahmen sind die Belastungen durch Umsetzung eines höheren Naturschutzniveaus im Wald stets auch abhängig von den gesellschaftlichen Ansprüchen und wirtschaftlichen Rahmenbedingungen. Änderungen sind hier im Laufe der 200-jährigen Simulationsperiode durchaus möglich, konnten in den Berechnungen jedoch nicht berücksichtigt werden.

Schlussendlich wäre die Erhöhung des Waldnaturschutzniveaus dann rational, wenn der zusätzliche Nutzen durch Naturschutzmaßnahmen über den Kosten liegen würde. Der monetär bewertete Nutzen und der Zeitpunkt seines Eintreffens sind jedoch derzeit noch nicht bekannt.

\subsection{Dritter Artikel „Abschätzung der holzbasierten Wertschöpfung und Beschäftigung in} stofflichen Wertschöpfungsketten“ und vierter Artikel: „Wertschöpfungsanalyse von Waldflächen und Rohholz"

Der Artikel „Abschätzung der holzbasierten Wertschöpfung und Beschäftigung in stofflichen Wertschöpfungsketten" wurde von Dr. Björn Seintsch und Lydia Rosenkranz geschrieben und 2014 in der Fachzeitschrift „Holz-Zentralblatt“ veröffentlicht. Der Artikel „Wertschöpfungsanalyse von Waldflächen und Rohholz" wurde von Lydia Rosenkranz und Dr. Björn Seintsch geschrieben und im Diskussionspapier Nr. 1405 „Vergleichende Bewertung der Nutzung von Biomasse“ (HAVERKAMP et al. 2014) des Departments für Agrarökonomie und Rurale Entwicklung der Universität Göttingen veröffentlicht. Er ist ein Anwendungsbeispiel für die im dritten Artikel vorgestellte Bewertungsmethode. Das Bewertungskonzept beider Artikel wurde von beiden Autoren, unter Federführung von Dr. Björn 
Seintsch, entwickelt. Lydia Rosenkranz führte die Datenaufnahmen und die Berechnungen durch. Dr. Björn Seintsch und Lydia Rosenkranz schrieben die Artikel gemeinsam.

\subsubsection{Zusammenfassung des dritten Artikels}

Ziel dieser Studie war die Entwicklung einer einfachen Methode zur Ermittlung der bundesdurchschnittlichen holzbasierte Wertschöpfung und Beschäftigung in den Branchen der ersten und zweiten Holzverarbeitungsstufe und hierauf aufbauend für holzbasierte Wertschöpfungsketten. Hierfür wurde der Ansatz von SEINTSCH (2011) zur Abschätzung der holzbasierten Wertschöpfung und Beschäftigung in der ersten Holzverarbeitungsstufe herangezogen. Damit kann die Wertschöpfung und Beschäftigung eines Wirtschaftszweigs, durch den Einsatz einer durchschnittlichen Einheit holzbasierter Rohstoffe, Halb- und Fertigwaren, für ein Bezugsjahr berechnet werden. Hierfür wird die Kostenstrukturerhebung (StBA: F 4 R 4.3) mit der Material- und Wareneingangsstatistik (StBA: F 4 R 4.2.4) des Statistischen Bundesamtes verknüpft. In der Kostenstrukturstatistik erscheinen jährlich Informationen zu Bruttoproduktionswert, Bruttowertschöpfung, Bruttoproduktionsgewinn und Beschäftigung der Unternehmen. Die vierjährlich erscheinenden Material- und Wareneingangsstatistik liefert Informationen über die Anschaffungskosten der Vorleistungen differenziert nach Warengruppen.

Im Ergebnis können mit dieser Methode u.a. die Ausgaben für Eingänge und Verbrauch an Roh-, Hilfs- und Betriebsstoffen sowie an Handelswaren für alle Vorleistungspositionen differenziert berechnet werden.

Durch die Integration der beiden Statistiken liegen jedoch zunächst nur Informationen zu den Anschaffungskosten der höher aggregierten Warengruppen vor. Mit weiteren amtlichen Statistiken und Studien wurde daher das Preis- und Mengengerüst dieser Warengruppen abgeschätzt. Weiterführend konnten auf dieser Basis Holzrohstoffkosten sortimentsspezifisch differenziert für die einzelnen Wirtschaftszweige ermittelt werden. Auf Basis der Bruttowertschöpfung, der Beschäftigungszahlen und der Holzrohstoffkosten sind Berechnungen der Wertschöpfung und Beschäftigung einer Einheit holzbasierter Rohstoffe für die Wirtschaftszweige der ersten Holzverarbeitungsstufe möglich. Ergänzend dazu wurden für den Wirtschaftsbereich Forstwirtschaft Kennzahlen zur Wertschöpfung und Beschäftigung pro Einheit Rohholz aus der Forstlichen Gesamtrechnung (FGR) abgeleitet.

Im Rahmen der eigenen Studie wurde die Methode von SEINTSCH (2011) für das Jahr 2010 aktualisiert und auf die zweite Holzverarbeitungsstufe übertragen. Hierfür wurde zunächst der Wert einer Einheit holzbasierter Rohstoffe, Halb- und Fertigwaren der Warengruppen der Material- und Wareneingangsstatistik für das Jahr 2010 im Durchschnitt berechnet. Durch die Material- und Wareneingangsstatistik liegen jedoch nur absolute Warenwertangaben zu Anschaffungskosten der einzelnen Warengruppen vor. Daher wurde unterstellt, dass die wert- und mengenmäßigen Struktur des Wareneingangs der holzverarbeitenden Wirtschaftszweige in der Material- und Wareneingangsstatistik der 
tiefer differenzierten Produktionsstatistik des Verarbeitenden Gewerbes (StBA F 4 R 3.1) entspricht. Die unterschiedlichen Mengeneinheiten holzbasierter Produkte der Produktionserhebungen wurden mittels eigener Annahmen einheitlich in Kubikmeter umgerechnet. Weiterhin wurden pauschale Transportkosten aufgeschlagen. Auf dieser Basis wurde mengen- und preisgewichtet der durchschnittliche Wert einer Einheit holzbasierter Rohstoffe, Halb- und Fertigwaren der Wirtschaftszweige in der Material- und Wareneingangsstatistik bestimmt.

Die abgeschätzten durchschnittlichen Werte einer Einheit holzbasierter Vorleistungen wurden in Beziehung zur Bruttowertschöpfung und Beschäftigung der holzverarbeitenden Wirtschaftszweige gesetzt. Im letzten Arbeitsschritt wurde die Bruttowertschöpfung und Beschäftigung exemplarisch für vier idealisierte Holzwertschöpfungsketten, inklusive der Forstwirtschaft, berechnet. Dabei wurde idealtypisch unterstellt, dass die Wirtschaftsbereiche linear miteinander vernetzt sind, d.h. dass die holzbasierten Vorleistungen eines Wirtschaftszweigens immer nur an einen anderen Wirtschaftszweig weitergegeben werden. In der realen Wirtschaftspraxis sind die Wirtschaftsbereiche jedoch auf unterschiedlichsten Ebenen vernetzt.

Der hier entwickelte Ansatz bietet eine Möglichkeit, die Wertschöpfung und Beschäftigung nicht nur für einzelne Wirtschaftszweige, sondern für ganze holzbasierte Wertschöpfungsketten abzuschätzen. So betrug die Bruttowertschöpfung pro Kubikmeter holzbasierter Vorleistungen in der Wertschöpfungskette "Holzhaus" im Jahr $2010273 €$ und die Beschäftigung 5,5 Vollzeitäquivalente. Für die Wertschöpfungskette "Verpackung" betrug die Bruttowertschöpfung $146 € / \mathrm{m}^{3}$ und die Beschäftigung 3,0 Vollzeitäquivalente/ $\mathrm{m}^{3}$. Für die Wertschöpfungskette „Papier“ betrug die Bruttowertschöpfung $2.647 € / \mathrm{m}^{3}$ und die Beschäftigung 34,1 Vollzeitäquivalente/ $\mathrm{m}^{3}$.

\subsubsection{Zusammenfassung des vierten Artikels}

Die Umsetzungsebene zahlreicher politischer Ziele sind Regionen. Auf regionaler Ebene werden FFHMaßnahmen implementiert, Nationalparke ausgewiesen und Entscheidungen hinsichtlich der stofflichen und energetischen Holznutzung getroffen. Für die Bewertung von konkurrierenden Wald- und Holznutzungsoptionen und für Wertschöpfungsanalysen in Regionen ist die Datenbasis unzureichend oder aufwendig zu erheben. Ziel dieser Studie ist daher die Entwicklung eines einfachen Verfahrens zur Folgenabschätzung der Auswirkungen von Nutzungskonkurrenzen (hier: Auswirkung veränderten Holzeinschlags auf die Wertschöpfung und Beschäftigung) auf regionaler Ebene.

Grundlage des hier vorgestellten Verfahrens ist der in Kapitel 2.3.1 dargestellte Ansatz zur Abschätzung der holzbasierten Wertschöpfung und Beschäftigung in den Wirtschaftszweigen der ersten und zweiten Holzverarbeitungsstufe und darauf aufbauend in holzbasierten Wertschöpfungsketten.

Für diesen Ansatz wurde zunächst die Bruttowertschöpfung und Beschäftigung für die Forstwirtschaft und die erste Holzverarbeitungsstufe pro Einheit holzbasierter Vorleistungen nach dem im 
Artikel „Abschätzung der holzbasierten Wertschöpfung und Beschäftigung in stofflichen Wertschöpfungsketten“ (Kapitel 2.3.1) beschriebenen Verfahren für das Jahr $2006^{8} \mathrm{im}$ Bundesdurchschnitt bewertet. Diese durchschnittlichen Kennzahlen wurden dann auf zwei Fallbeispielsregionen übertragen. Dazu wurde mit dem StRUGHOLTZ-EngleRT-Simulationsmodell (siehe Kapitel 2.1.1) eine Abschätzung des regionalen Holzeinschlags durchgeführt. Als Eingangsdaten lagen nur die regionalspezifischen Flächen der Holzartengruppen Buche, Eiche, Fichte, Kiefer und Douglasie sowie deren durchschnittliche Produktionszeiten vor. Weitere Eingangsdaten, wie z.B. zur Altersklassenverteilung oder Bewirtschaftungsvarianten waren nicht verfügbar. Daher wurden für die weiteren Steuergrößen Durchschnittswerte unterstellt. Auf Grundlage des regionalspezifischen Holzeinschlags aus den Szenarien und der berechneten Wertschöpfung und Beschäftigung pro Einheit holzbasierter Vorleistungen können dann Abschätzungen der Auswirkungen veränderten Holzeinschlags auf Regionen getätigt werden. Da die energetische Holznutzung in den amtlichen Statistiken nicht oder nicht ausreichend erfasst ist, wurde hier auf die Ergebnisse von SCHWEINLE (2012) zurückgegriffen. Somit wurde ein Vergleich der Wertschöpfung und Beschäftigung zwischen der energetischen und stofflichen Holznutzung ermöglicht.

Für die beiden Fallbeispielsregionen, Göttinger Land und Thüringer Ackerebene, wurden im vorliegenden Artikel neben dem Szenario „Status quo“" ${ }^{9}$ die beiden Verwendungsszenarien „Hausbrand“: (Nutzung des gesamten Einschlags für den Hausbrand) und „Erhöhung des Laubholzanteils der Sägeindustrie ${ }^{10}$ (z.B. Förderung der stofflichen Verwendung) sowie die beiden Waldnutzungsszenarien "Stilllegung von 5 \% der Waldfläche“ (z.B. Umsetzung der Biodiversitätsstrategie) und „Stilllegung von Laubaltholzflächen über 120 Jahre“ (z.B. Umsetzung der FFH-Richtlinie) berechnet. Für die Region Göttinger Land liegt die berechnete Bruttowertschöpfung bei einer Fortführung des „Status quo“ bei $62 € / \mathrm{m}^{3}$ und eine Beschäftigung von 211 Vollzeitäquivalenten. Würde das Verwendungsszenario „Hausbrand“ umgesetzt, so reduziert sich die Bruttowertschöpfung auf $54 € / \mathrm{m}^{3}$ und die Beschäftigung auf 138 Vollzeitäquivalente. Würde das Waldnutzungsszenario „Stilllegung von $5 \%$ der Waldfläche" umgesetzt so reduzierte sich die Wertschöpfung auf $59 € / \mathrm{m}^{3}$ und die Beschäftigung auf 201 Vollzeitäquivalente. Bei einer Umsetzung des Szenarios „Erhöhung des Laubholzanteils der Sägeindustrie" würde sich in der Region "Göttinger Land" die Bruttowertschöpfung auf $66 € / \mathrm{m}^{3}$ und die Beschäftigung auf 251 Vollzeitäquivalenten erhöhen.

Für die holzverarbeitenden Wirtschaftszweige wird somit aufgezeigt, welche Wertschöpfung und Beschäftigung durch eine Einheit Rohholz oder holzbasierter Vorleistungen generiert werden kann. Bei einer Erhöhung der Rohholzpreise oder der Transportkosten für holzbasierte Vorleistungen (z.B.

\footnotetext{
${ }^{8}$ Zum Zeitpunkt dieser Studie lag die Material- und Wareneingangsstatistik für das Jahr 2010 noch nicht vor.

${ }^{9}$ Hierbei wurde eine Nutzung von Laubholz zu $20 \%$ als Stamm- und $80 \%$ als Energieholz sowie Nadelholz zu $80 \%$ als Stamm- und $20 \%$ als Energieholz unterstellt.

${ }^{10}$ Für das Szenario „Erhöhung des Laubholzanteils der Sägeindustrie“ wurde unterstellt, dass das in der Region vorhandenen Laubholz zu $50 \%$ als Stammholz und zu $50 \%$ als Energieholz genutzt wird.
} 
bei einer Erweiterung des Beschaffungsradius) ist zu erwarten, dass sich die Wertschöpfung und Beschäftigung pro Einheit holzbasierter Vorleistungen reduziert. Außerdem können auf Basis dieser Zahlen Rückschlüsse auf die Auswirkungen verminderter Holzmengen gezogen werden.

\subsubsection{Kritische Diskussion und Einordnung in den thematischen Rahmen}

Mit den vorliegenden Artikeln wurde eine neue Methode zur Berechnung von Kennzahlen zur Abschätzungen der wirtschaftlichen Auswirkungen veränderten Holzeinschlags auf die Holzwirtschaft und auf Regionen vorgestellt. Die Ergebnisse liegen in plausibler Größenordnung zu den Ergebnissen der Input-Output-Rechnungen von DIETER (2008) zum Holz- und Papiergewerbe.

Kritisch zu beurteilen ist, dass die Berechnungen teilweise auf einfachen Annahmen beruhen, da statistische Daten nur unzureichend vorhanden sind. So wurde unterstellt, dass der Wareneingang nach der Material- und Wareneingangsstatistik in seiner wert- und mengenmäßigen Struktur den Produktionserhebungen des Statistischen Bundesamtes entspricht. Bei der Produktionserhebung handelt es sich jedoch um eine Vollerhebung während die Material- und Wareneingangsstatistik auf einer Stichprobenerhebung des Verarbeitenden Gewerbes beruht. Zwischen den beiden Statistiken könnten sich daher Abweichungen der wert- und mengenmäßigen Struktur ergeben. Ferner werden in der Produktionserhebung die Mengen der produzierten holzbasierten Waren in verschiedenen Einheiten $\left(m^{3}, m^{2}, m, t, \ldots\right)$ angegeben, was zu Schwierigkeiten bei der Abschätzung der produzierten Mengen aus den Produktionserhebungen führte. Zur besseren Vergleichbarkeit mussten diese zunächst einheitlich in Kubikmeter umgerechnet werden. Dies geschah auf Basis von Literaturrecherchen und eigenen Annahmen. Folglich entsprechen die berechneten Werte nicht vollständig der Realität. Es ist jedoch keine andere geeignete Statistik zur Berechnung von Produktionsmengen verfügbar, weshalb auf die Produktionserhebungen des StBA zurückgegriffen werden musste. Weiterhin wurde der Außenhandel nicht berücksichtigt.

Im vierten Artikel wurde die Methode zur Abschätzung von Wertschöpfung und Beschäftigung auf Basis einer Einheit holzbasierter Rohstoffe mit der Methode zur Berechnung des Holzeinschlags mit dem STRUGHOLTZ-EngLeRT-Simulationsmodell verknüpft. Anhand von zwei Fallbeispielsregionen wurde verdeutlicht, welche Auswirkungen eine Verminderung von Holzeinschlag oder eine Veränderung der Holznutzung in Regionen für die Wertschöpfung und Beschäftigung haben kann. Für die Berechnungen wurden bundesweite Durchschnittszahlen auf einzelne Regionen übertragen und pauschale Annahmen zur regionalen Waldbewirtschaftung getroffen. Auch für die Szenarien wurden pauschale Annahmen unterstellt. Restriktiv für die Umsetzung der Szenarien, insbesondere für das Szenario „Erhöhung des Laubholzanteils“ wirken hier die Qualität des Holzes, die Nachfrage nach den verschiedenen Holzprodukten sowie die Verarbeitungskapazitäten in der Region. 
Interregionale Verflechtungen blieben unberücksichtigt. Örtliche Verhältnisse konnten ebenfalls aufgrund schlechter Datenlage nicht berücksichtigt werden. Die Berechnungen bieten somit nur orientierende Größenordnungen. Ein weiterer Ansatz wäre die Aufnahme des tatsächlichen regionalen „Industriebestands“ auf Basis vorhandener Daten (z.B. Industrie- und Handelskammer oder Sonderauswertungen der Umsetzsteuerstatistik) und die Übertragung der bundesdurchschnittlichen Kennzahlen auf die konkreten Verhältnisse der Regionen. Die Daten der Industrie- und Handelskammer und die regionalspezifische Daten der Umsatzsteuerstatistik waren aufgrund des Datenschutzes jedoch für die eigenen Berechnungen nicht verfügbar.

\subsection{Fünfter Artikel „Decomposition analysis of changes in value added. A case study of the sawmilling and wood processing industry in Germany"}

Dieser Artikel wurde von Lydia Rosenkranz, Dr. Björn Seintsch und Prof. Dr. Matthias Dieter geschrieben und 2015 in der Fachzeitschrift „Forest Policy and Economics" nach einem double blind peerreview veröffentlicht. Das Berechnungsverfahren der Dekompositionsanalyse wurde von Prof. Dr. Matthias Dieter entwickelt. Prof. Dr. Matthias Dieter schrieb den Textteil zur Dekompositionsmethode. Lydia Rosenkranz nahm die Daten auf, führte die Berechnungen durch, validierte den theoretisch entwickelten Dekompositionsansatz, schrieb den Methodenteil zur Vorbereitung der Dekompositionsanalyse und schrieb den Rest des Artikels federführend. Dr. Björn Seintsch war Co-Autor.

\subsubsection{Zusammenfassung}

Ziel der Studie war die Entwicklung einer Methode zur Ausweisung von unterschiedlichen Effekten für Wertschöpfungsänderungen. Mit der Methode können Veränderungen der Wertschöpfung in Wirtschaftszweigen analysiert werden.

Wertschöpfungsänderungen zwischen zwei Betrachtungsjahren/-zeitpunkten können durch Änderungen der (nominalen) Produktpreise, der (nominalen) Vorleistungspreise, der Outputmenge und den Anteilen von Vorleistung und Wertschöpfung durch Outsourcing oder Offshoring entstehen. Im Rahmen einer Dekompositionsanalyse wurden Wertschöpfungsänderungen in zwei holzverarbeitenden Wirtschaftszweigen daher in einen 1) Produktpreiseffekt, 2) Vorleistungspreiseffekt, 3) Wachstumseffekt (unterteilt nach Input und Output) und 4) Struktureffekt zerlegt. Die Wertschöpfungsänderungen ergeben sich hier, wie in der klassischen Definition, aus Änderungen des Outputs abzüglich des Inputs. Die in Anlehnung an den Paasche-Index entwickelte Formel erlaubt eine Fokussierung auf die reinen Mengeneffekte.

Als Fallbeispiele wurden die Wirtschaftszweige 1610 „Säge-, Hobel- und Holzimprägnierwerke“ (Sägeindustrie) und 1621 „Herstellung von Furnier-, Sperrholz-, Holzfaser- und Holzspanplatten“ (Holzwerkstoffindustrie), als Berechnungszeitraum die Jahre 2002 bis 2006 sowie 2006 bis 2010 ausge- 
wählt. Als Grundlage für die Berechnungen wurden zunächst die Bruttowertschöpfung sowie Preise, Mengen und Wachstumsfaktoren insgesamt und für die einzelnen Vorleistungen für die beiden Wirtschaftszweige in den Jahren 2002, 2006 und 2010 bestimmt. Mit der Methode von SEINTSCH (2011) konnten die Ausgaben für einzelne Vorleistungen im Bezugsjahr berechnet werden. Preisänderungen der Vorleistungen wurden über eine Auswertung der Produktpreisindizes des Statistischen Bundesamts bestimmt. Auf dieser Grundlage konnten gewichtete durchschnittliche Wachstumsfaktoren der Inputpreise je Betrachtungsjahr ermittelt und überdies der Einfluss von Preis- und Mengenänderungen einzelner holzbasierter Vorleistungen auf die Wertschöpfung der Wirtschaftszweige abgeschätzt werden. Für die Kalkulation der Outputmengen- und preise wurde aufgrund fehlender statistischer Datengrundlagen unterstellt, dass der Output der Wirtschaftszweige von den Warengruppen 161„,Holz gesägt, gehobelt“ bzw. 1621 “Furnier-, Sperrholz-, Holzfaser- und Holzspanplatten” bestimmt wurde. Dementsprechend wurden Preisindizes dieser beiden Warengruppen für die Berechnungen verwendet.

Zwischen 2002 und 2006 stieg die Wertschöpfung in der Sägeindustrie. Die Wertschöpfung der Holzwerkstoffindustrie sank im selben Zeitraum. Zwischen 2006 und 2010 sank die Bruttowertschöpfung in beiden Wirtschaftszweigen. Mittels der Dekompositionsanalyse konnte festgestellt werden, dass die Änderung der Wertschöpfung zwischen den Jahren 2002 und 2006 für die Sägeindustrie vor allem durch den Produktpreiseffekt bedingt wurde. Zudem überstieg der Wachstumseffekt des Outputs den Wachstumseffekt des Inputs. Zwischen 2006 und 2010 reduzierte sich der Produktpreiseffekt im Vergleich zur Vorperiode um die Hälfte (52\%). Ebenso sanken der Wachstums- und der Struktureffekt, was auf eine geringe Marktnachfrage hindeutet. Im Gegensatz dazu stieg der Vorleistungspreiseffekt im Vergleich zur Vorperiode um 133 \% und ist somit der Hauptfaktor für die negative Veränderung der Bruttowertschöpfung. In der Holzwerkstoffindustrie wurde die negative Veränderung der Bruttowertschöpfung zwischen 2002 und 2006 hauptsächlich durch den Struktureffekt bedingt. Dies ist ein Hinweis auf hohe Outsourcing- und Offshoring-Aktivitäten. Zwischen 2006 und 2010 sank zusätzlich der Produktpreiseffekt um rund die Hälfte im Vergleich zur Vorperiode. Auch der Strukturund der Wachstumseffekt nahmen ab.

In der Sägeindustrie konnte, unter Ausschluss von Preisänderungen, ein Zusammenhang zwischen dem Holzinput und dem Wachstum nachgewiesen werden. Während der Wachstumseffekt zwischen 2002 und 2006 um 25,4 \% zu- und zwischen 2006 und 2010 um $15 \%$ abnahm, nahm der Input von „022 Rohholz" zwischen 2002 und 2006 um 37 \% zu und zwischen 2006 und 2010 um 13 \% ab. In der Holzwerkstoffindustrie konnte dieser Zusammenhang nicht nachgewiesen werden.

Im Rahmen der Studie konnte weiterhin die hohe Bedeutung von Rohholz und holzbasierten Vorleistungen für die Sägeindustrie aufgezeigt werden. Die Vorleistungspositionen korrespondieren mit den Änderungen der Gesamtvorleistungsmengen und -preise: Im Jahr 2006 konnten die Änderungen von 
Ausgaben für Vorleistungen weitgehend auf Mengenänderungen und im Jahr 2010 auf Preisänderungen zurückgeführt werden. Dies deutet auf einen Zusammenhang zwischen den Änderungen der Gesamtvorleistungen mit den Änderungen holzbasierter Vorleistungen hin.

\subsubsection{Kritische Diskussion und Einordnung in den thematischen Rahmen}

Im Rahmen der Studie wurde erstmalig eine Dekompositionsanalyse zur Bewertung von Wertschöpfungseffekten entwickelt und erfolgreich an zwei Wirtschaftszweigen der Holzwirtschaft angewendet. Sie trägt zur Erklärung von Wertschöpfungsänderungen in der holzverarbeitenden Industrie bei.

Die Qualität der Ergebnisse ist grundsätzlich abhängig von der Datengrundlage. In der Kostenstrukturerhebung wird der Verbrauch an Roh-, Hilfs- und Betriebsstoffen, nicht jedoch der Anteil einzelner Vorleistungen differenziert angegeben. Hilfsweise wurde für die eigenen Rechnungen, zur Aufwertung der Datengrundlage und zur Analyse des Einflusses einzelner Vorleistungen, die Material- und Wareneingangsstatistik in die Kostenstrukturerhebung des Statistischen Bundesamtes nach der Methode von SEINTSCH (2011) integriert. Dadurch wurde eine Abschätzung von gewichteten Durchschnittswerten und -mengen, deren Änderungen zwischen den Bewertungsjahren und der Bedeutung holzbasierter Vorleistungen für die Wirtschaftszweige ermöglicht. Eine höhere Differenzierung der verwendeten Statistiken wäre für zukünftige Studien wünschenswert.

Weiterhin ist aufgrund des vierjährigen Erscheinens der Material- und Wareneingangsstatistik keine durchgehende Analyse im betrachteten Zeitraum möglich, sondern es konnten nur Veränderungen zwischen den Jahren 2002, 2006 und 2010 bewertet werden. Die Wertschöpfungseffekte und ihr Zusammenhang mit den Marktentwicklungen konnten in den Zwischenjahren daher nicht analysiert werden. Eine Übertragung des Anteils der Vorleistungen der Material- und Wareneingangsstatistik auf die Kostenstrukturstatistik in den Zwischenjahren wurde für die eigenen Arbeiten jedoch als nicht plausibel erachtet. Für genauere Analysen wäre ein jährliches Erscheinen der Material- und Wareneingangsstatistik wünschenswert.

Die Ergebnisse der Studie spiegeln die Entwicklungen des Holzmarktes in den Jahren von 2002 bis 2010 jedoch gut wider. Die Sägeindustrie profitierte zwischen 2002 und 2010 von den günstigen Marktbedingungen, insbesondere der hohen Aufnahmekapazität in- und ausländischer Absatzmärkte, und konnte dementsprechend hohe Umsätze erzielen. In der Folge konnten Kapazitäten ausgebaut und Outputmengen erhöht werden. Ferner profitierte die Sägeindustrie auch von den Preissteigerungen einstmals niedrigpreisiger Sägenebenprodukte, wie z.B. Sägemehl, zur energetischen Verwendung. In der Holzwerkstoffindustrie reduzierte sich dagegen die Wertschöpfung im selben Zeitraum durch hohe Ausgaben für Vorleistungen, bedingt durch Nutzungskonkurrenzen um den Rohstoff Holz. Besonders vormalig niedrigpreisige Holzsortimente wie Holz schlechter Qualität und Sägenebenprodukte, beides wichtige Rohstoffe der Holzwerkstoffindustrie, erfuhren im Zuge der zu- 
nehmenden Nachfrage nach Energieholz starke Preissteigerungen. Darüber hinaus nahm das Einschlagspotential der heimischen Nadelhölzer in den letzten Jahren ab, was zu weiteren Preissteigerungen geführt haben dürfte. Die Änderung der Wertschöpfung und des Wachstumseffekts beider Wirtschaftszweige zwischen 2006 und 2010 ist insbesondere auf die geringe Nachfrage und schlechte Auftragslage sowie die Reduktion von Produktionskapazitäten, ausgelöst durch die Weltwirtschaftskrise von 2007 bis 2009, zurückzuführen.

Mit der im Rahmen dieser Studie entwickelten Methode konnten Gründe für die Wertschöpfungsentwicklung der beiden Holzindustriezweige verdeutlicht werden. Wertschöpfungsänderungen ausschließlich mit Änderungen des Holzinputs zu erläutern, greift jedoch zu kurz. Auch andere Entwicklungen wie Outsourcing, Substitutionseffekte, Marktverhältnisse, Konsumententrends, Saisoneffekte, nationaler und internationaler Wettbewerb und staatliche Einschränkungen können hier eine Rolle spielen. Diese Faktoren drücken sich in den Preisen aus und wurden im Rahmen dieser Studie nicht gesondert interpretiert. 


\section{Diskussion und Schlussfolgerungen}

Die MCPFE fordert in der Helsinki-Resolution H1 „General Guidelines for the Sustainable Management of Forests in Europe“ von 1993 die nachhaltige Nutzung von Waldressourcen und Waldflächen in Europa. Die Resolution beschreibt die nachhaltige Bewirtschaftung der Wälder als „stewardship and use of forests and forest lands in a way, and at a rate, that maintains their biodiversity, productivity, regeneration capacity, vitality and their potential to fulfil, now and in the future, relevant ecological, economic and social functions, at local, national, and global levels, and that does not cause damage to other ecosystems" (MCPFE 1993:1). Die Wichtigkeit dieser Balance aus ökologischem, ökonomischem und sozialem Nutzen des Waldes wird auch in der Waldstrategie 2020 betont (BMELV 2011). Für einen verantwortungsvollen Umgang mit der Ressource Wald müssen die Auswirkungen eigenen Handelns bekannt sein. Das umfasst auch die Auswirkungen von Änderungen der Waldbewirtschaftung. Folgenabschätzungen für den Wald und für dessen ökologische, ökonomische und soziale Funktionen sind wegen der langen Lebenszyklen und forstlichen Produktionszeiten eine besondere Herausforderung.

Für politische Folgeabschätzungen der Auswirkungen einer veränderten Waldbewirtschaftung, z.B. zur Bedürfnisbefriedigung von einzelnen gesellschaftlichen Gruppen, bedarf es u.a. ökonomischer Bewertungen ihrer Wirkung auf die Forst- und Holzwirtschaft. Die vorgestellten eigenen wissenschaftlichen Arbeiten sollen hierzu einen Beitrag liefern. Kern dieser Arbeit sind die Nutzungskonkurrenzen zwischen Naturschutz und Holznutzung.

Bewertungen der Auswirkungen von Waldnaturschutzmaßnahmen auf die Forstwirtschaft

Die Bereitstellung öffentlicher Güter des Waldes, wie die Umsetzung naturschutzfachlicher Maßnahmen, können wirtschaftliche Belastungen für Waldbesitzer zur Folge haben, die kompensiert werden müssen (MÖHRING und MESTEMACHER 2009). Bei den Belastungen kann es sich um Mehraufwendungen, z.B. durch Monitoring oder Biotoppflege, oder Mindererträge (Opportunitätskosten) durch Nutzungsverzicht oder Nutzungsextensivierung handeln.

Zur Berechnung von kurz- und langfristigen Einschlags- und Deckungsbeitragsveränderungen bedingt durch naturschutzfachliche Maßnahmenplanungen wurde in den Studien von ROSENKRANZ et al. (2014) und RosenkRANZ und SEINTSCH (2015) das StRUGHOLTZ-EngleRT-Simulationsmodell verwendet und im Rahmen der eigenen Arbeit weiterentwickelt. Die derzeitige Modellversion lässt jedoch Raum für weitere Verbesserungsansätze. So lassen sich mit dem Modell bislang keine Mischbestände simulieren. Stattdessen werden die den Forsteinrichtungsdaten entnommenen Baumartenanteile nach Holzartengruppen zu Reinbeständen zusammengefasst. Unterschiedliche Wachstumsdynamiken der Baumarten in Mischbeständen können auf diese Weise nicht berücksichtigt werden. Die dem Modell unterliegenden Waldwachstumsfunktionen basieren auf den parametrisierten Ertragstafeln von 
SMALTSCHINSKI (1998). Baumwachstumsbeeinflussende Standortsbedingungen, wie z.B. Wasserhaushalt, Bodenart und Standortsklima, spiegeln sich zwar indirekt in den Ertragsklassen wider, können aber nicht einzeln beeinflusst werden. Die Ertragsklasse kann vor Beginn der Simulation gewählt, im Laufe der Simulationsperiode jedoch nicht mehr verändert werden. Auch die Wahrscheinlichkeit von Kalamitäten im Laufe der 200-jährigen Simulationszeit muss bereits vor Beginn der Simulation abgeschätzt und festgelegt werden. Im Hinblick auf Änderungen des Baumwachstums und Änderungen der Baumartenzusammensetzung bedingt durch den Klimawandel wären dynamische klimasensitive Anpassungsmöglichkeiten im Laufe der Simulationsperiode im Modell sinnvoll.

Des Weiteren können mit dem Modell bislang keine Holzpreis- und Holzerntekostenänderungen im 200-jährigen Simulationszeitraum berechnet werden. Die Holzerntekosten und Holzpreise müssen vor Beginn der Berechnung festgelegt und können während der Simulation nicht verändert werden. Holzpreise unterliegen jedoch kalamitäts- oder nachfragebedingten Änderungen, daher sind statische Holzpreise im Simulationszeitraum nicht realistisch. Auf der anderen Seite ist die Abschätzung der Holzpreisentwicklungen über einen derartig langen Zeitraum schwierig. Hier könnten beispielsweise Monte-Carlo-Simulationen im Modell eingebaut werden. Aus einer Bandbreite unterstellter zukünftiger Holzpreise und -erlöse könnten mit der Monte-Carlo-Simulation eine Vielzahl unterschiedlicher Szenarien der Deckungsbeitragsentwicklung berechnet werden. Anschließend könnten anhand von Annahmen über zukünftige Rahmenbedingungen (z.B. Klimawandel, Nadelholzknappheit durch Umbau zu Laubholz, Änderung gesellschaftlicher Zielsetzungen,...) Abschätzungen über die Wahrscheinlichkeit des Auftretens der berechneten Szenarien durchgeführt werden.

Eine weitere mögliche Weiterentwicklung des STRUGHOLTZ-ENGLERT-Simulationsmodells ist die Bewertung von Ökosystemleistungen, beispielsweise für Nutzenbewertungen oder für Kompensationsforderungen. So könnten u.a. zusätzlich zu den vom Modell berechneten Holzvorräten und den Holzeinschlagsmengen der $\mathrm{CO}_{2}$-Gehalt des Holzes berechnet werden, um Klimaschutzleistungen des Waldes und der Forstwirtschaft über bestimmte Zeiträume zu bewerten. Oder es könnten mehr Baumarten in das Modell integriert werden, z.B. um Waldumbaumaßnahmen zur Verbesserung der Waldästhetik (für den Tourismus) und/oder der Baumartenvielfalt (für den Naturschutz) abbilden zu können.

Die eigenen Berechnungen zeigten, dass bei einer Umsetzung von FFH-Maßnahmenplanungen deutliche Ertragseinbußen für die privaten, kommunalen und staatlichen Fallbeispielsforstbetriebe entstehen (ROSENKRANZ et al. 2014): Die Deckungsbeitragsverluste lagen im Durchschnitt über den 200jährigen Simulationszeitraum bei rund $40 € /$ ha/Jahr auf Buchenwald-Lebensraumtypflächen. Zum Vergleich: AICHHOLZ et al. (2013) ermittelten Deckungsbeitragsverluste durch Umsetzung von FFHMaßnahmenplanungen von 32 - $36 € /$ ha/Jahr über alle Waldlebensraumtypen. MöHRING, STRATMANN und GERST (in: SEINTSCH et al. 2012: 91 ff) ermittelten Einkommensverluste (Annuitäten) von $67 € /$ ha/Jahr für die Umsetzung von FFH-Maßnahmen in 10 Fallbeispielsforstbetrieben. Die unter- 
schiedlichen Ergebnisse sind auf verschiedene Bewertungsansätze (komparativ-statisch vs. dynamisch) und Bewertungszeiträume sowie auf teilweise unterschiedliche Eingangsdaten zurückzuführen.

An den Ergebnissen der Studie wird deutlich, dass eine Aufrechterhaltung naturschutzfachlicher Leistungen auf Teilflächen in der Forstwirtschaft zu großen Verlusten führen kann, die durch staatliche Transferzahlungen ausgeglichen werden müssten. Setzt sich zudem der derzeitige Trend der steigenden holzerntekostenfreien Erlöse weiter fort (ERMISCH et al. 2014), ist eine weitere Steigerung der Opportunitätskosten für die Umsetzung naturschutzfachlicher Maßnahmenplanungen für Waldbesitzer zu erwarten.

Die Höhe der Belastungen in den Fallbeispielsforstbetrieben erwies sich in hohem Maße abhängig von der betriebsindividuellen Naturalausstattung und den Eigentümerzielen und variierte zwischen den verschiedenen Betrieben stark. Folglich wären individuelle Kompensationszahlungen, abhängig von der Belastung der jeweiligen Betriebe, die gerechteste Lösung, sie dürfte jedoch an den hohen Transaktionskosten der betriebsindividuellen Bewertung scheitern. Praktikabler wären pauschale Kompensationszahlungen. Die Methode und die Ergebnisse der Studie von RoSENKRANZ et al. (2014) könnten zur Entwicklung fairer Kompensationsprogramme für Forstbetriebe, so z.B. zur Erstellung von „Belastungstabellen“ nach naturaler Ausstattung und Naturschutzmaßnahmen, herangezogen werden.

Derzeit werden Forderungen zu einer Segregation der Waldfunktionen auf Teilflächen in Deutschland laut (HöLTERMANN 2013). Dies würde eine Abkehr vom bisherigen forstpolitischen Leitbild der multifunktionalen Waldbewirtschaftung bedeuten. Im Rahmen der Segregation auf Teilflächen werden auch die Erhöhung von Stilllegungsflächen (z.B. Nationalparks, Wildnisgebiete) sowie eine Erweiterung der Flächen mit extensiver Waldbewirtschaftung gefordert. Hierzu gehört zum Beispiel die Forderung nach einer Erhöhung des Laubholzanteils mit längeren Produktionszeiträumen sowie von höheren Altholzanteilen, was einer Produktion von Starkholz gleichzusetzen ist. In der Forstwirtschaft hat die Umsetzung der naturschutzfachlichen Forderungen mittlerweile in vielen Waldbaurichtlinien der Landesforstverwaltungen Eingang gefunden (z.B. MLUR BRANDENBURG 2004, ML NIEDERSACHSEN 2004, LANDESFORSTEN RHEINLAND-Pfalz (Jahr unbekannt)). Der Trend zu höheren Laubholzanteilen wird bereits deutlich: während das Laubholz insgesamt in Deutschland einen Anteil von rund $44 \%$ an der Waldfläche hat, so hat es an der ersten Altersklasse bereits einen Anteil von $58 \%$ (BWI 2012b). In Deutschland wird jedoch hauptsächlich mittelstarkes Nadelholz verarbeitet. Mit der Erhöhung des Anteils von starkem Laubholz auf großer Fläche wird folglich an den Bedürfnissen der holzverarbeitenden Industrie vorbeiproduziert. Für die Deckung der steigenden Nachfrage nach Energieholz könnte sich die Erhöhung der Laubholzanteile dagegen positiv auswirken. 
Die Auswirkungen einer möglichen Umsetzungsvariante der Segregation der Waldfunktionen auf Teilflächen, wurden von ROSENKRANZ und SEINTSCH (2015) erstmalig für die gesamte deutsche Forstwirtschaft aufgezeigt. Würden die naturschutzfachlichen Maßnahmen, wie unter den in der Studie getroffenen Annahmen umgesetzt, so würde der derzeitige Nettounternehmensgewinn im Wirtschaftsbereich Forstwirtschaft erheblich reduziert und der Gesamteinschlag der Bundesrepublik um durchschnittlich rund $15 \%$ zurückgehen.

Häufig wird argumentiert, dass sich Naturschutzleistungen durch Steigerung der Einnahmen durch Tourismus regionalwirtschaftlich positiv auswirken ${ }^{11}$. So berechneten JOB et al. (2005) für den Nationalpark Müritz einen Nettoumsatz von rund 12 Mio. $€ / J a h r$ und für den Naturpark Altmühltal einen Nettoumsatz von rund 18 Mio. $€ / J a h r$. Die Mehreinahmen durch Tourismus müssten jedoch bundesweit berechnet und den Verlusten der Forstwirtschaft und der ersten und zweiten Holzverarbeitungsstufe gegengerechnet werden. Die Methoden und Ergebnisse der in dieser Arbeit vorgestellten Studien könnten hierfür herangezogen werden.

\section{Bewertungen der Auswirkungen von reduziertem Holzaufkommen auf die Holzwirtschaft}

Dem durch die Umsetzung naturschutzfachlicher Forderungen reduzierten inländischen Holzeinschlag steht die stetig steigende Nachfrage nach Holz zur energetischen und stofflichen Verwendung gegenüber. Durch die Dekompositionsanalyse (ROSENKRANZ et al. 2015) wurde der enge Zusammenhang zwischen holzbasierten Vorleistungen, der Wertschöpfung und dem Wachstum der Sägeindustrie deutlich. Eine Erhöhung der Holzpreise aufgrund von Knappheiten dürfte sich daher direkt negativ auf die Wertschöpfung der Unternehmen auswirken. Außerdem ist zu erwarten, dass sich ein verminderter Holzeinschlag auf das Wachstum der Sägeindustrie negativ auswirkt.

Holzverarbeitende Betriebe, insbesondere jene der ersten Verarbeitungsstufe, sind in hohem Maße von der regionalen Verfügbarkeit von Rohholz abhängig. Fallen für die Holzindustrie lokale oder regionale Holzbeschaffungsmärkte weg (z.B. durch Waldflächenstilllegung) oder verringert sich das Holzaufkommen (z.B. durch Extensivierung), so könnte es regional zu Versorgungsengpässen kommen. Die Holzbeschaffungsradien müssten sich erhöhen, was wiederum zu einer Erhöhung der Transportkosten und einer Verschärfung von Nutzungskonkurrenzen in anderen Regionen führen würde. Aufgrund der hohen Transportkosten dürfte die erste Verarbeitungsstufe die Versorgungsengpässe nur bedingt über den Außenhandel oder interregionalen Handel kompensieren können.

Durch die Umsetzung der naturschutzfachlichen Forderungen, den zunehmenden Knappheiten des Rohstoffs Holz und den daraus folgenden Holzpreissteigerungen können sich Änderungen der Mengen- und Sortimentsstruktur von Rohholz, der Rohholzbeschaffungsstruktur und Rohholzkosten sowie Veränderungen von Wachstum, Wertschöpfung und Beschäftigung für die Holzindustrie ergeben.

\footnotetext{
${ }^{11}$ z.B. Studien über die Wirtschaftlichkeit von Nationalparks auf regionaler Ebene: Job et al. (2005), Berger (2011)
} 
Unter den wirtschaftlichen Rahmenbedingungen erscheinen daher Kapazitätsanpassungen und Betriebsschließungen der ersten Verarbeitungsstufe wahrscheinlich. Aufgrund der Vernetzung der holzverarbeitenden Industrie ist weiterhin zu vermuten, dass sich eine Reduktion des Holzeinschlags auch auf Wertschöpfung und Beschäftigung der zweiten Holzverarbeitungsstufe auswirkt. Ob in der Folge in der zweiten Verarbeitungsstufe mehr Halbwaren importiert werden oder ob ebenfalls Kapazitätsanpassungen erfolgen, muss geprüft werden.

Mit einem Anteil an der deutschen Volkswirtschaft von 2,2 \% der Bruttowertschöpfung und 3,0 \% der Beschäftigung (BECHER 2014) ist die volkswirtschaftliche Bedeutung des Clusters Forst und Holz vergleichsweise gering. Aus volkswirtschaftlicher Sicht wären die Opportunitätskosten für den Naturschutz möglicherweise tragbar. Kapazitätsanpassungen und Betriebsschließungen aufgrund mangelnder Verfügbarkeit von Rohholz oder Erhöhung von Holztransportkosten könnten sich jedoch negativ auf die regionale Beschäftigungssituation und damit indirekt auch (geringfügig) auf die Nachfrage nach anderen Gütern in Deutschland auswirken (vgl. ROSENKRANZ und SEINTSCH 2014, SEINTSCH und ROSENKRANZ 2015).

DiETER (2009) zeigt für das Problem des abgeschöpften inländischen Nadelrohholzaufkommens drei Lösungsmöglichkeiten auf: eine zumindest auf Teilflächen stärkere Ertragsorientierung, die zusätzliche Beschaffung von Holz außerhalb des Waldes oder durch erhöhten Import sowie eine Anpassung der unterschiedlichen Nutzungsansprüche an die verfügbare Waldfläche. Gemäß DIETER (2009) ist zwar der Außenhandel mit Holz- und Holzprodukten stark angestiegen, die geringsten Wachstumsraten weisen dabei jedoch Rohholz und Restholz auf. Er folgert, dass exportstarke Länder zwar mit Holzfertigwaren handeln, Rohholz jedoch größtenteils für den Eigenbedarf nutzen und dass eine Erhöhung der Holzmenge, die aus dem Ausland bezogen wird, daher nur begrenzt oder gar nicht möglich ist. Im Hinblick auf die Abhängigkeit der rohholzaufnehmenden Industrie von der lokalen Verfügbarkeit von Holz ist weiterhin zu erwarten, dass, insbesondere bei Betrieben, die in großen Mengen Massensortimente verarbeiten, Rohholzimporte aufgrund der Transportkosten wirtschaftlich kaum umsetzbar sein dürften. Auch eine stärkere Ertragsorientierung dürfte aus naturschutzfachlichen Gründen (Trend zu mehr Laubholz und Einschlagsreduktionen, insbesondere im öffentlichen Wald) vielerorts schwer umsetzbar sein. In der Folge wird eine Anpassung der unterschiedlichen Nutzungsansprüche an die verfügbare Waldfläche erreicht werden müssen.

\section{Ausblick}

Mit der vorliegenden Arbeit wurden die Auswirkungen der Umsetzung von Naturschutzmaßnahmen auf die Forst- und Holzwirtschaft bewertet. Ein besonderer Fokus lag dabei auf den naturalen und wirtschaftlichen Auswirkungen verminderten Holzeinschlags auf die Forst- und Holzwirtschaft. Für eine vollständige Kosten-Nutzenbewertung bedarf es jedoch auch Nutzenbewertungen von natur- 
schutzfachlichen Maßnahmen, zum einen hinsichtlich ihrer Wirksamkeit für die angestrebte Zielerreichung (Erhalt/Erhöhung der Biodiversität) und zum anderen hinsichtlich des monetären Nutzens für Forstbetriebe. Im Rahmen der eigenen Arbeiten konnte langfristig kein monetärer Nutzen für Forstbetriebe aus der reinen Umsetzung von Naturschutzmaßnahmen abgeleitet werden. Monetärer Nutzen könnte jedoch über ausreichend hohe, d.h. nicht nur den Ertragsverlust abdeckende, Kompensationszahlungen erzeugt werden. Studien zur Wirksamkeit von naturschutzfachlichen Maßnahmen wurden aufgrund der Unsicherheit bezüglich ihrer langfristigen Wirkungen nicht durchgeführt bzw. lagen nicht vor. Hierzu wäre weitere Forschung wünschenswert. 


\section{Literaturverzeichnis}

AICHHOLZ R, SCHMACK S und HARTEBRODT C (2012): Naturschutzfachliche Einschränkungen der Forstwirtschaft in FFH-Gebieten. Schlussbericht zum Verbundvorhaben „Auswirkungen von naturschutzfachlichen Anforderungen auf die Forst- und Holzwirtschaft“; Teilvorhaben 1: „Restricted Forest Management" (ReForMa). Kurzfassung. Forstliche Versuchs- und Forschungsanstalt Baden-Württemberg - Abt. Forstökonomie. $76 \mathrm{~S}$.

BECHER G (2014): Clusterstatistik Forst und Holz. Tabellen für das Bundesgebiet und die Länder 2000 bis 2012. Thünen Working Paper 32, Hamburg. $105 \mathrm{~S}$.

BERGEN V, LÖWENSTEIN W und OLSCHEWSKI R (2013): Forstökonomie - Volkswirtschaftliche Ansätze für eine vernünftige Umwelt- und Landnutzung. Verlag Franz Vahlen $\mathrm{GmbH}$, München. $477 \mathrm{~S}$.

BERGER R (2011): Konzeption und Wirtschaftlichkeit des Nationalparks Teutoburger Wald/ Eggegebirge. Roland Berger Strategy Consultants. URL: http://de.nationalpark-senneeggegebirge.de/download/gutachten/. Abgerufen am 15.09.2015.

BFN (Bundesamt für Naturschutz) (2009): Zeitplan zum Verfahren der Gebietsausweisung und der Berichterstattung im Rahmen des Netzwerkes Natura 2000. URL: https://www.bfn.de/0316_meldeverfahren.html

BFN (2012): Waldlebensraumtypen in Deutschland. Sonderauswertung des BfN im Auftrag des Verbundforschungsprojektes FFH-Impact. (Stand: Mai 2012)

BFN (2015): Zum Stand der Umsetzung von Natura 2000 in Deutschland. https://www.bfn.de/0316_gebiete.html\#c5409. Abgerufen am 17.07.2015. Letzte Änderung am 21.03.2014

BMVEL (Bundesministerium für Ernährung, Landwirtschaft und Verbraucherschutz) (2004): Verstärkte Holznutzung. Zugunsten von Klima, Lebensqualität, Innovationen und Arbeitsplätzen (Charta für Holz). $23 \mathrm{~S}$.

BMELV (Bundesministerium für Ernährung, Landwirtschaft) (2009): Aktionsprogramm „Energie für morgen - Chancen für ländliche Räume“. Berlin. $17 \mathrm{~S}$.

BMELV (2011) Waldstrategie 2020: Nachhaltige Waldbewirtschaftung - eine gesellschaftliche Chance und Herausforderung, Bundesministerium für Ernährung, Landwirtschaft und Verbraucherschutz, Bonn. $36 \mathrm{~S}$.

BMEL (2014): Der Wald in Deutschland. Ausgewählte Ergebnisse der dritten Bundeswaldinventur.

BMEL (2015a): Buchführungsergebnisse Forstwirtschaft Ergebnisse 2013. Privatwaldbetriebe nach Baumarten.http://www.bmelv-statistik.de/de/testbetriebsnetz/buchfuehrungsergebnisseforstwirtschaft/. Abgerufen am 02.10.2015.

BMEL (2015a): Buchführungsergebnisse Forstwirtschaft Ergebnisse 2013. Körperschaftwaldbetriebe nach Baumarten.

http://www.bmelv-statistik.de/de/testbetriebsnetz/buchfuehrungsergebnisseforstwirtschaft/. Abgerufen am 02.10.2015. 
BMELV und BMU (2010): Nationaler Biomasseaktionsplan für Deutschland. Beitrag der Biomasse für eine nachhaltige Energieversorgung. Berlin. $32 \mathrm{~S}$.

BMU (Bundesministerium für Umwelt, Naturschutz und Reaktorsicherheit) (2007): Nationale Strategie zur biologischen Vielfalt. Reihe Umweltpolitik. $170 \mathrm{~S}$.

BNATSCHG (Bundesnaturschutzgesetz) vom 29. Juli 2009 (BGBI. I S. 2542), das zuletzt durch Artikel 4 Absatz 100 des Gesetzes vom 7. August 2013 (BGBI. I S. 3154) geändert worden ist

BORCHERS J (2010): Segregation versus Multifunktionalität in der Forstwirtschaft. Forst und Holz 65, Heft 7/8: 44-49

Borchert H, Wellhausen K, Röder H, Steinbeis E, Fiedler S, Weber-BlaschKe G, Jentsch A, Mai W, Bauer J, BLENK M und KOLLERT W (2008): Clusterstudie Forst und Holz in Bayern 2008. Cluster-initiative Forst und Holz Bayern (Hrsg.). Freising

BÖSCH M, JOCHEM D, WeIMAR H und Dieter M (2015a): Physical input-output accounting of the wood and paper flow in Germany. Resources, Conservation and Recycling 94: 99-109

BÖSCH M, WEIMAR H und DIETER M (2015b): Input-output evaluation of Germany's national cluster of forest-based industries. European Journal of Forest Research 134(5): 899-910

BUNDESTAG (2009): Gesetz zur Förderung Erneuerbarer Energien im Wärmebereich (ErneuerbareEnergien-Wärmegesetz - EEWärmeG). $8 \mathrm{~S}$.

BWALDG (BUNDESWALDGESETZ) vom 2. Mai 1975 (BGBI. I S. 1037), Zuletzt geändert durch Artikel 1 des Gesetzes vom 31. Juli 2010 (BGBI. I S. 1050). 13 S.

BürgerbeWegung UnSER TeUtoburger Wald-Kein Nationalpark IN TeUtobuRger Wald /EgGe/SenNe (2012): Kein Nationalpark in Teutoburger Wald/Egge/Senne. URL:

https://www.openpetition.de/petition/online/kein-nationalpark-in-teutoburger-wald-eggesenne. Abgerufen am 21.07.2015

BURKHARDT R, ROBISCH F und ECKHARD S unter Mitarbeit der Mitglieder der LANA-FCK-Kontaktgruppe und des Bund-Länder Arbeitskreises „FFH-Berichtspflichten: Wald“ (2004): Umsetzung der FFH-Richtlinie im Wald. Gemeinsame bundesweite Empfehlungen der Länderarbeitsgemeinschaft Naturschutz (LANA) und der Forstchefkonferenz (FCK). Natur und Landschaft 2004/7: 316-323

BWI (2012a): Thünen-Institut, Dritte Bundeswaldinventur - Ergebnisdatenbank, https://bwi.info, Aufruf am: 24.06.2015, Auftragskürzel: 77Z1JI_L101of_2012, Archivierungsdatum: 2014-8-13 16:42:23.590, Überschrift: 1.02 Waldfläche [ha] nach Land und Eigentumsart, Filter: $\mathrm{Jahr}=2012$

BWI (2012b): Thünen-Institut, Dritte Bundeswaldinventur - Ergebnisdatenbank, https://bwi.info, Aufruf am: 21.07.2015, Auftragskürzel: 77Z1JI_L235of_2012_bi, Archivierungsdatum: 2014-610 16:7:59.927, Überschrift: Waldfläche (gemäß Standflächenanteil) [ha] nach Baumaltersklasse und Baumartengruppe (rechnerischer Reinbestand) 
BWI (2012c): Thünen-Institut, Dritte Bundeswaldinventur - Ergebnisdatenbank, https://bwi.info, Aufruf am: 21.09.2015, Auftragskürzel: 77V1PI_L637mf_0212_bi, Archivierungsdatum: 20148-5 14:24:44.730, Überschrift: Veränderung des Anteils an der Holzbodenfläche [\%-Punkte] nach Land und Baumartengruppe (rechnerischer Reinbestand)

DieTER M (2005): Holzbilanzen 2002, 2003 und 2004 für die Bundesrepublik Deutschland. Hamburg: Institut für Ökonomie der Bundesforschungsanstalt für Forst- und Holzwirtschaft, Arbeitsbericht 2005/3. 36 S.

DIETER M (2006): Holzbilanzen 2004 und 2005 für die Bundesrepublik Deutschland. Arbeitsbericht des Instituts für Ökonomie 2006/2, Hamburg : Johann-Heinrich-von-Thünen-Institut. 19 S.

DIETER M (2007): Holzbilanzen 2005 und 2006 für die Bundesrepublik Deutschland. Arbeitsbericht des Instituts für Ökonomie 2007/2, Hamburg : Johann-Heinrich-von-Thünen-Institut. 21 S.

DIETER M (2008): Analyse der Wertschöpfung durch Holznutzung aus gesamtwirtschaftlicher Perspektive. Allgemeine Forst- und Jagdzeitung 179, Jahrgang 10/11: 202-207

DIETER M (2009): Volkswirtschaftliche Betrachtung von holzbasierter Wertschöpfung in Deutschland. Landbauforschung - vTI Agriculture and Forestry Research Sonderheft: 37-46.

DIETER M (2010): Produktion, Weiterverarbeitung und Handel von Holz aus gesamtwirtschaftlicher Perspektive - eine quantitative Analyse der Forst- und Holzwirtschaft auf Grundlage sektoraler Statistiken. Habilitationsschrift zur Erlangung der venia legendi im Fachbereich Forstökonomie. Georg-August Universität Göttingen, Fakultät für Forstwissenschaften und Waldökologie. $177 \mathrm{~S}$.

DIETER M (2013): Nachhaltigkeit der Forstwirtschaft auf dem Prüfstand. AFZ-DerWald 68(11): 19-22

DiETER M und BORMANN K (2009): Fiskalische Effekte von Holznutzung im intersektoralen Vergleich. Allgemeine Forst- und Jagdzeitung 180 (7/8): 170-175

Dieter M und ENGleRT H (2007): Competitiveness in the global forest industry sector: an empirical study with special emphasis on Germany. European Journal of Forest Research 126: 401-412

DIETER M und ENGLERT H (2009): Zur Wettbewerbsfähigkeit der europäischen Holzwirtschaft - eine Constant-Market-Share-Analyse. Schweizerische Zeitschrift für Forstwesen 160 (12): 375-383

DieTER M und SEINTSCH B (2012): Änderung der Wettbewerbsfähigkeit der Holz- und Papierwirtschaft in Deutschland auf Grund zunehmender Knappheit an Nadelholz. Allgemeine Forst- und Jagdzeitung 188 (5/6): 116-128

DIETER M, ELSASSER P, KÜPPERS J-G und SEINTSCH B (2008): Rahmenbedingungen und Grundlagen für eine Strategie zur Integration von Naturschutzanforderungen in die Forstwirtschaft. Arbeitsbericht des Instituts für Ökonomie der Forst- und Holzwirtschaft Hamburg vTI 2008/02. 36 S.

DIETERICH V (1953): Forstwirtschaftspolitik. Eine Einführung. Paul Parey, Hamburg, Berlin, 398 S. 
DöbBeler H, AlBert M, SCHMidt M, NAGeL J und SCHRÖDER J (2011): BWINPro. Programm zur Bestandesanalyse und Prognose. Handbuch zur gemeinsamen Version von BWINPro und BWINPro-S. Version 6.3. Nordwestdeutsche Forstliche Versuchsanstalt und TU Dresden. $127 \mathrm{~S}$.

ErLbeck R, HASeder I und Stinglwagner G (1998): Das Kosmos Wald- und Forstlexikon. Stuttgart: Kosmos. $880 \mathrm{~S}$.

ERMISCH N., SEINTSCH B und DIETER M (2013): Holzartengruppe Fichte 2003 bis 2011 konstant mit höchstem Erlösbeitrag. AFZ-DerWald 68(23):6-9

ERMISCH N., SEINTSCH B und DÖg M (2014): Ergebnisse des Testbetriebsnetzes Forst des BMEL. AFZDerWald 23: 19-20

EUROPÄISCHE KOMMISSION (2003): Natura 2000 und der Wald. ,Herausforderungen und Chancen'. Auslegungsleitfaden. Europäische Kommission, GD Umwelt, Referat Natur und biologische Vielfalt Abteilung Forst- und Landwirtschaft. Luxemburg: Amt für amtliche Veröffentlichungen der Europäischen Gemeinschaften. 120 S.

FAUSTMANN M: Berechnung des Werthes, welchen Waldboden sowie noch nicht haubare Holzbestände für die Waldwirtschaft besitzen. Allgemeine Forst und Jagdzeitung 2/1849: 441-455

FFH-RICHTLINIE (Richtlinie 92/43/EWG des Rates zur Erhaltung der Lebensräume sowie der wildlebenden Tiere und Pflanzen) vom 21 Mai 1992, Abl. Nr. L 206, S. 7.

FOREST EUROPE (2011): State of Europe's Forests 2011: Status and Trends in Sustainable Forest Management in Europe. Oslo

FORSTMASCHINEN-PROFI (2010): „SChallende Ohrfeige für Waldbesitzer“. Forstfachverlag GmbH \& Co. $\mathrm{Kg}$, Schleeßen

GIBSON H, HANLEY N, und WRIGHT R (2004): An economic assessment of the costs and benefits of Natura 2000 sites in Scotland. Project Report. Scottish Government. 75 S.

GREENPEACE (2012): Nationalpark Teutoburger Wald jetzt! URL: https://www.greenpeace.de/themen/waelder/schutzgebiete/nationalpark-teutoburgerwald-jetzt. Abgerufen am 21.07.2015

HAMPICKE U (1991): Naturschutzökonomie. Uni-Taschenbücher 1650, Verlag Eugen Ulmer, Stuttgart, $341 \mathrm{~S}$.

HANEWINKEL M (2011): Multifunktionalität des Waldes. Eidgenössische Forschungsanstalt für Wald, Schnee und Landschaft. Forum für Wissen 2011: 7-14

HANSEN, J., 2012. Der WaldPlaner - Ein Entscheidungsunterstützungssystem für den Forstbetrieb. Lecture Notes in Informatics Informationstechnologie für eine nachhaltige Landbewirtschaftung.Gesellschaft für Informatik, Bonn: 115-118

HARSCHE J Und JAENSCH K (2009): Regionalökonomische Bestimmungsgrößen für das Standortwahlverhalten der Holzwirtschaft und Papierindustrie in Hessen. Jahrbuch der Österreichischen Gesellschaft für Agrarökonomie, Band 18(3): 67-76. 
Haverkamp M, Henke S, Kleinschmit C, Möhring B, Müller H, Mubhoff O, Rosenkranz L, Seintsch B, SCHLOSSER K, und THEUVSEN L (2014): Vergleichende Bewertung der Nutzung von Biomasse: Ergebnisse aus den Bioenergieregionen Göttingen und BERTA. Diskussionspapier Department Agrarökonomie und Rurale Entwicklung, Universität Göttingen Nr. 1405: 22-26

HeLD C, GRULKE M und SEINTSCH B. (2008): Zur Wettbewerbsfähigkeit der Holzenergie : "SWOT-Analyse und zukünftige Rahmenbedingungen der 1 . Verarbeitungsstufe des bundesweiten Clusters Forst und Holz" : Teil 1. Holz Zentralblatt 134(6): 145-147

HöLteRMANN A (2013): Das 5 \%-Ziel: Begründung und Bedeutung. Holz-Zentralblatt Nr. 40: 977-978

IVANOV S und WEBSTER C (2010): Decomposition of economic growth in Bulgaria by industry. Journal of economic studies 37 (2), 219-227.

Job H, HARRER B, Metzler D und HaJizadeh-Alamdary D (2005): Ökonomische Effekte von Großschutzgebieten Untersuchung der Bedeutung von Großschutzgebieten für den Tourismus und die wirtschaftliche Entwicklung der Region. BfN-Skripten 135. 119 S.

Jochem D, Weimar H, Bösch M, Mantau U und Dieter M (2015a): Der Holzeinschlag - eine Neuberechnung: Ergebnisse der verwendungsseitigen Abschätzung des Holzeinschlags in Deutschland für 1995 bis 2013. Holz Zentralblatt 141(30):752-753

Jochem D, Weimar H, Bösch M, MANTAU U und Dieter M (2015b) Estimation of wood removals and fellings in Germany: a calculation approach based on the amount of used roundwood. European Journal of Forest Research 134(5): 869-888

KLEIN D, KIES U und SCHULTE A (2009): Regional employment trends of wood-based industries in Germany's forest cluster: a comparative shift-share analysis in post-reunification development. European Journal of Forest Research 128: 205-219.

KLOSE F und ORF S (1998): Forstrecht. Kommentar zum Waldrecht des Bundes und der Länder. Aschendorffs Juristische Handbücher Münster/Köln. 746 S.

Krott M, Julien B, Lammertz M, Barbier J-M, Jen S, Ballesteros M und De Bovis C (2000): Voicing Interests and Concerns: NATURA 2000: An ecological network in conflict with people. Forest Policy and Economics 1: 357-366

KÜPPERS J-G, und DIETER M (2008): Belastungen der Forstbetriebe aus der Schutz- und Erholungsfunktion des Waldes (2003 bis 2006). Arbeitsbericht des Instituts für Ökonomie der Forst- und Holzwirtschaft 04: 29 S.

LANDESFORSTEN RHEINLAND PFALZ (Jahr unbekannt): Qualifizieren - Dimensionieren. Waldbaustrategie. $20 \mathrm{~S}$.

Li C-Z, Kuuluvainen J, Pouta E, Rekola M und Tahvonen O (2004): Using choice experiments to value the Natura 2000 nature conservation programs in Finland. Environmental \& Resource Economics 29:361-374. 
LÜCKGE F-J, DISPAN J, HELD C und SEINTSCH B (2008): Zur Wettbewerbsfähigkeit der Holzindustrie: SWOT-Analyse und zukünftige Rahmenbedingungen der 1 . Verarbeitungsstufe des bundesweiten Clusters Forst und Holz (Teil II). Holz-Zentralblatt, 134. Jg., Nr. 10, S. 265-268

MANTAU U (2010): Rohstoffknappheit und Holzmarkt. In: Depenheuer, O. und Möhring, B. Hrsg.) (2010): Waldeigentum. Dimensionen und Perspektiven. Springer-Verlag Berlin Heidelberg: 139-147

MANTAU U (2012) Holzrohstoffbilanz Deutschland. Entwicklungen und Szenarien des Holzaufkommens und der Holzverwendung von 1987 bis 2015. Hamburg. $65 \mathrm{~S}$.

McGRegOR P und MCNICOLL I (2006): The Impact of Forestry on Output in the UK and its Member Countries. Regional Studies, Vol. 26.1: 69-79

MCPFE (Ministerkonferenz zum Schutz der Wälder in Europa) (1993): RESOLUTION H1 General Guidelines for the Sustainable Management of Forests in Europe. Second Ministerial Conference on the Protection of Forests in Europe 16-17 June 1993, Helsinki/Finland. $5 \mathrm{~S}$.

Meyer P, Menke N, Nagel J, Hansen J, Kawaletz H, PaAr U, Evers J (2009): Abschlussbericht des von der Deutschen Bundesstiftung Umwelt geförderten Projekts: Entwicklung eines Managementmoduls für Totholz im Forstbetrieb. Nordwestdeutsche Forstliche Versuchsanstalt: $110 \mathrm{~S}$.

MILANA, C., 1988. Constant-market-share: an analysis and index number theory. European Journal of Political Economy 4: 453-478.

ML NiederSACHSEN (Niedersächsisches Ministerium für den ländlichen Raum, Ernährung, Landwirtschaft und Verbraucherschutz) (2004): Langfristige ökologische Waldentwicklung. Richtlinien zur Baumartenwahl. Aus dem Walde - Schriftenreihe Waldentwicklung Niedersachsen Heft 54. $150 \mathrm{~S}$.

MLUR Brandenburg (Ministerium für Landwirtschaft, Umweltschutz und Raumordnung des Landes Brandenburg) (2004): Waldbau-Richtlinie 2004, „Grüner Ordner“ der Landesforstverwaltung Brandenburg Forst. $143 \mathrm{~S}$.

MÖHRING B und MESTEMACHER U (2009): Gesellschaftliche Leistungen de Wälder und der Forstwirtschaft und ihre Honorierung. Landbauforschung - vTI Agriculture and Forestry Research Sonderheft 327: $65-73$

MÖHRING B und RÜPING U (2006): Bewertungskonzept für forstliche Nutzungsbeschränkungen. Schriften zur Forstökonomie Band 32. J. D. Sauerländer's Verlag, Frankfurt am Main.

OCHS T, DUSCHL C und SEINTSCH B (2007): Rohstoffversorgung beim Nadelholz angespannt: Teil II der Studie „Regionalisierte Struktur- und Marktanalyser der 1. Verarbeitungsstufe der Holzwirtschaft". Holz-Zentralblatt, 133. Jg., Nr. 10: 318-320

Oesten G und ROEDER A (2002): Management von Forstbetrieben Band 1: Grundlagen, Betriebspolitik. Verlag Dr. Kessel, Remagen-Oberwinter. 373 S.

POLLEY H (2009): Wald in Schutzgebieten - ein Überblick. Landbauforschung - vTI Agriculture and Forestry Research Sonderheft 327: 75-82 
POSAVEC S, BELAAN K und LOVRIC M (2011): Model of compensation payment to the owners on Natura 2000 forest sites. Glas. šum. Pokuse, Zagreb 44:19-28

Pouta E, Rekola M, KuUluvainen J, TAhVonen O und LI C-Z (2000): Contingent valuation of the Natura 2000 nature conservation programme in Finland. Forestry 73 (2):119-128.

PRETZSCH H, BIBER P und DURSKÝ J (2002): The single tree-based stand simulator SILVA: construction, application and evaluation. Forest Ecology and Management 162:3-21.

ROSENKRANZ L und WIPPEL B (2012) Erhaltungszustandsbewertung und Maßnahmenplanung von FFHBuchen-Lebensraumtypen. AFZ Wald 67(12):8-9

ROSENKRANZ L, WIPPEL B, SEINTSCH B (2012): Teil 1: Umsetzung der FFH-Richtlinie im Wald in den Bundesländern. Arbeitsbericht des OEF, vTI, Hamburg $119 \mathrm{~S}$.

RosenkRanz L, SEINTSCH B, WIPPEL B, und Dieter M (2014): Income losses due to the implementation of the Habitats Directive in forests - Conclusions from a case study in Germany. Forest Policy and Economics 38:207-218

RosenKRANZ L, SEINTSCH B und DieTer M (2015): Decomposition analysis of changes in value added: a case study of the sawmilling and wood processing industry in Germany. Forest Policy and Economics 54:36-50

ROSENKRANZ L und SEINTSCH B (2015): Opportunitätskostenanalyse zur Implementierung des naturschutzorientierten Waldbehandlungskonzepts "Neue Multifunktionalität". Landbauforschung Appl Agric Forestry Res 65(3/4):145-160

RUPF H (1961): Wald und Mensch im Geschehen der Gegenwart. Allg. Forstzeitschrift - Der Wald 16: 545-546

Rüther B, Hansen J, Ludwig A, Spellmann H, Nagel J, Möhring B und Dieter M (2007): Clusterstudie Forst und Holz Niedersachsen. Nordwestdeutsche Forstliche Versuchsanstalt (Hrsg.). Göttingen

SAGL W (1995): Bewertung in Forstbetrieben. Pareys Studientexte 80, Blackwell Wissenschaftsverlag Berlin-Wien. $306 \mathrm{~S}$.

Schmidt-Bleibtreu B, Hofmann H, Hopfauf A (2011): Grundgesetz, Kommentar, 12. Aufl. 2011, Neuwied: Luchterhand.

SCHWEINLE, J. (2012): Wertschöpfungsanalyse der energetischen Nutzung von Holz, in: Arbeitsbericht, Nr. 2012/02, vTI - Institut für Ökonomie der Forst- und Holzwirtschaft (Hrsg.), Hamburg.

SeEgMüLler S (2005): Die Forst-, Holz- und Papierwirtschaft in Rheinland-Pfalz. Forschungsanstalt für Waldökologie und Forstwirtschaft. Trippstadt.

SEINTSCH B (2010): Entwicklungen des Clusters Forst und Holz zwischen 2000 und 2007. Ergebnisse und Tabellen für das Bundesgebiet und die Länder. Arbeitsbericht des Instituts für Ökonomie der Forst- und Holzwirtschaft 2010 / 2. 74 S. 
SEINTSCH B (2011): Stellung der Holzrohstoffe in der Kostenstruktur des Holz- und Papiergewerbes in Deutschland. vTI, Arbeitsbericht des Instituts für Ökonomie der Forst- und Holzwirtschaft 03/2011. Hamburg. 106 p.

SEINTSCH B und ROSENKRANZ L (2014): Erneutes Rekordergebnis der Forstwirtschaft : auch ein Grund zur Nachdenklichkeit? - Ergebnisse der Forstwirtschaftlichen Gesamtrechnung 2012. Holz Zentralblatt 140(13):316-317

SEINTSCH B und ROSENKRANZ L (2015A): Wertschöpfung und Beschäftigung durch Holznutzung : Abschätzung der holzbasierten Wertschöpfung und Beschäftigung in stofflichen Wertschöpfungsketten. Holz-Zentralblatt 141(5):110-112

SEINTSCH B und ROSENKRANZ L (2015b): Ausblick: Was wäre wenn? Der Wald der Zukunft? - Ökonomische Betrachtungen zum Waldnaturschutz . Vortrag im Rahmen des Dialogforums Waldnaturschutz und Biodiversität im Rahmen der Tagung zur Waldstrategie 2020, 01. -02. Juli 2015, Berlin. URL: https://waldstrategie2020.info/veranstaltungsbeitraege/

SEINTSCH B und WEIMAR H (2013): Holzbilanzen 2010 bis 2012 für die Bundesrepublik Deutschland. Thünen Working Paper 9, Hamburg: Johann Heinrich von Thünen-Institut. $37 \mathrm{~S}$.

Seintsch B, Rosenkranz L, Englert H, Dieter M, Wippel B, Becker G, Stratmann J, Gerst J und Möhring B (2012a) FFH-Impact: Teil 2: Auswirkungen von FFH-Maßnahmenplanungen auf Forstbetriebe. Hamburg: Institut für Ökonomie der Forst- und Holzwirtschaft, 144 p, Arbeitsbereich Inst Ökon Forst Holzwirtsch vTI 2012/05. 150 S.

SEINTSCH B, WIPPEL B und ROSENKRANZ L (2012b) Befragung von Forstbetrieben zu Auswirkungen von FFH-Maßnahmenplanungen. AFZ Wald 67(20):10-11

SIPPEL A (2007). Forstliche Nutzung in FFH-Gebieten. Situationsanalyse und Perspektiven. Fachstudie erstellt durch die Forstliche Versuchs- und Forschungsanstalt Baden Württemberg im Auftrag der Bundesforschungsanstalt für Forst- und Holzwirtschaft. Freiburg, Forstliche Versuchsund Forschungsanstalt Baden-Württemberg: $86 \mathrm{~S}$.

SMALTSCHINSKI T (1998) Regionale Waldwachstumsprognose. Schriftenreihe Freiburger Forstliche Forschung. Universitätsdruckerei Freiburg (121 pp.).

SPEIDEL G (1967): Forstliche Betriebswirtschaftslehre. Eine Einführung. Verlag Paul Parey, Hambugr und Berlin. 289 S.

TBA (Statistisches Bundesamt) (2007): Gliederung der Klassifikation der Wirtschaftszweige, Ausgabe 2008 (WZ 2008). Arbeitsunterlage. Wiesbaden

STBA: Außenhandel nach Waren und Ländern (F7 R2), Wiesbaden, jährlich

STBA: Index der Erzeugerpreise der Produkte des Holzeinschlags aus den Staatsforsten (F17 R1),Wiesbaden (jährlich)

STBA: Index der Erzeugerpreise gewerblicher Produkte Deutschland (F17 R2), Wiesbaden (jährlich) 
STBA: Kostenstruktur der Unternehmen des Verarbeitenden Gewerbes sowie des Bergbaus und der Gewinnung von Steinen und Erden (F 4 R 4.3), Wiesbaden (jährlich)

STBA: Material- und Wareneingangserhebung im Verarbeitenden Gewerbe sowie im Bergbau und in der Gewinnung von Steinen und Erden (F 4 R 4.2.4), Wiesbaden (jährlich)

STBA: Produktionserhebungen (F 4 R 3.1), Wiesbaden (jährlich)

STRUGHOLTZ A (2010): Ein forstbetriebliches Simulationsmodell zur ökonomischen Bewertung strategischer forstlicher Produktionsentscheidungen. Masterarbeit, Fakultät für Forstwissenschaften und Waldökologie der Georg-August- Universität, Göttingen. 77 S.

StUTTGARTER ZeITUNG (2011): Geplanter Nationalpark erhitzt die Gemüter. URL: http://www.stuttgarter-zeitung.de/inhalt.nordschwarzwald-geplanter-nationalpark-erhitztdie-gemueter.99fb1190-195a-4dad-a122-3a55e89d2107.html. Abgerufen am 21.07.2015

SUDA M und PUKALL K (2014): Multifunktionale Forstwirtschaft zwischen Inklusion und Extinktion (Essay). Schweizerische Zeitung für Forstwesen 165/11: 333-338

TAZ (Tageszeitung) (2013): Naturschutz im Schwarzwald. Bewohner gegen Nationalpark. URL: http://www.taz.de/Naturschutz-im-Schwarzwald/!5066040/. Abgerufen am 21.07.2015

THOROE C (2009): Wald im Wandel - gesamtgesellschaftliche Herausforderungen. Landbauforschung vTI Agriculture and Forestry Research Sonderheft: 5 - 9

Thoroe C, Dieter M, Elsasser P, Englert H, KüPpers J-G und Roering H-W (2003): Untersuchungen zu den ökonomischen Implikationen einer Präzisierung der Vorschriften zur nachhaltigen, ordnungsgemäßen Forstwirtschaft bzw. von Vorschlägen zur Konkretisierung der Guten fachlichen Praxis in der Forstwirtschaft. Arbeitsbericht des Instituts für Ökonomie 2003 / 3. 73 S.

VOGELSCHUTZRICHTLINIE (Richtlinie 79/409/EWG des Rates über die Erhaltung der wildlebenden Vogelarten) vom 2. April 1979, ABI. L 103 vom 25.4.1979. 27 S.

WAGNER S Und JÖNSSON A (2001): Einschränkungen der Waldbewirtschaftung durch Naturschutzauflagen am Beispiel des europäischen Schutzgebietssystems Natura 2000. Rechtsfragen und monetäre Bewertung. Gutachten im Auftrag der Arbeitsgemeinschaft Deutscher Waldbesitzerverbände e. V. (AGDW): $130 \mathrm{~S}$.

WEIMAR H (2014): Holzbilanzen 2012 und 2013 für die Bundesrepublik Deutschland. Hamburg: Johann Heinrich von Thünen-Institut, Thünen Working Paper 31. $37 \mathrm{~S}$.

WWF (World Wide Fund for Nature) (2013): Geplanter Nationalpark Nordschwarzwald: Diskussion um eine große Chance. URL: http://www.wwf.de/themen-projekte/waelder/nationalparknordschwarzwald/. Abgerufen am 21.07.2015 
Anhang: wissenschaftliche Artikel 
1. Rosenkranz L, SeintsCh B, Wippel B, und Dieter M (2014): Income losses due to the implementation of the Habitats Directive in forests - Conclusions from a case study in Germany. Forest Policy and Economics 38:207-218 


\title{
Income losses due to the implementation of the Habitats Directive in forests - Conclusions from a case study in Germany
}

\author{
Lydia Rosenkranz ${ }^{\text {a, } *}$, Björn Seintsch ${ }^{a}$, Bernd Wippel ${ }^{b}$, Matthias Dieter ${ }^{a}$ \\ a Thünen-Institute of International Forestry and Forest Economics, Hamburg, Germany \\ ${ }^{\mathrm{b}}$ UNIQUE forestry and land use GmbH Freiburg, Germany
}

\section{A R T I C L E I N F O}

Article history:

Received 30 April 2013

Received in revised form 8 October 2013

Accepted 22 October 2013

Available online 11 November 2013

\section{Keywords:}

Nature conservation

Forest enterprise

Harvesting restriction

Evaluation

Economic impact

Costs

\begin{abstract}
A B S T R A C T
In the context of implementing the Habitats Directive (92/43/EEC) approximately $17 \%$ of the German forest area was designated in "Special Areas of Conservation (SAC)". Amongst these there are many beech forests which were not subject to a special protection status before.

Management plans, containing measures for the protection of SACs, are just being developed. These measures may cause restrictions to forest management leading to losses of income. Our study aimed to analyse natural and economic impacts of the implementation of the Habitats Directive which could, e.g. be used as a basis for designing compensation schemes.

In discussion with operational managers it became clear that the measures most restrictive to forest enterprises were small-area land set-aside, restrictions in choice of tree-species and maintenance of a sufficient share of mature stands.

The impact of those nature protection measures on case-study forest enterprises was evaluated using an excelbased simulation model which enabled the calculation of, e.g., income losses based on enterprise individual data for a given simulation-period.

The main factors influencing income losses were age-class distributions, management practices and objectives of forest enterprises. Annual income losses for the enterprises averaged 31 to $39 € /$ ha.
\end{abstract}

(c) 2013 Elsevier B.V. All rights reserved.

\section{Introduction}

The Fauna-Flora-Habitats Directive (92/43/EEC, FFH- or Habitats Directive) aims "to maintain or restore, at favourable conservation status, natural habitats and species of wild fauna and flora of Community interest" (Habitats Directive 92/43/EEC, Art. 2). It was introduced in the EU in 1992 as part of the European Natura 2000 nature conservation network.

The responsibility for implementing the Habitats Directive lies with the EU Member States. Each State is obliged to designate "Special Areas of Conservation (SACs)" for the protection of natural habitat types and species listed in the annexes of the Directive and to ensure the required conservation measures (COM, 2003). The Habitats Directive mainly focuses on protection objectives and hardly any direct requirements for forest management can be withdrawn (Winkel et al., 2009). It is the task of the EU Member States to concretise the abstract conservation objectives and measures (here called FFH-measures) for each of the respective SACs in terms of so-called "management" or "maintenance and development" plans (Habitats Directive 92/43/EEC, Art.6).

\footnotetext{
* Corresponding author at: Thünen Institute of International Forestry and Forest Economics Leuschnerstraße 91, D-21031 Hamburg, Germany.

E-mail address: lydia.rosenkranz@ti.bund.de (L. Rosenkranz).
}

The Habitats Directive was included in the German Federal Nature Conservation Act in 1998. The authority for the concretisation and implementation of the FFH-measures lies, in a federalist state like Germany, mainly with the Bundeslaender. In consequence, the concepts, the methods and the status of implementation within the Laender are diverse. There are, for example, varying threshold values as well as practices for surveying the conservation status of SACs, on the basis of which FFH-measures are formulated. For this reason the FFH-measures in the Laender can be different for the same object of protection (Rosenkranz et al., 2012).

Natura 2000 areas today cover about $24 \%$ of the German woodland (Polley, 2009). Approximately 1.8 Mio ha (17\%) of the total German forest area is located within SACs. There are 18 different forest habitat types in Germany (BfN, 2012). Amongst them there are about 0.55 Mio ha of beech forests which have not been subject to a special protection status before (Sippel, 2007). These forests are not only of great importance for nature conservation goals but also for forest management and the German policy objectives in the fields of economics, energy, and climate protection.

There are yet hardly any research results about the impacts of the implementation of the Habitats Directive on forestry. With the ongoing process of concretising the abstract conservation objectives in management plans the impacts on forest management have now become 
assessable. Requirements like the conservation of habitat trees, dead wood and mature forests as well as the protection of habitat-specific natural species composition (cf. BfN, 2006) are likely to have impacts on forest management. Reductions of the amount of harvested timber and of the income of forest enterprises are to be expected.

Also, the forest enterprise characteristics, which especially influence impacts of FFH-measures, need to be determined. Generally it is to be expected, that FFH-measures in single forest enterprises can have a different degree of impact, depending on the respective forest enterprise's objectives, and the currently exercised or planned forest management.

Some Bundeslaender already apply compensation instruments such as nature conservation contracts (e.g. Bavaria and Hesse) or lump sum payments (e.g. North Rhine Westphalia and Baden-Württemberg). Others are just now developing compensation instruments. Thus, knowledge of the natural and economic impacts of FFH-measures and of factors enhancing the loss of income could prove to be helpful in this development process.

\section{Research objectives}

Against this backdrop, the joint research project "Impacts of nature protection requirements on forestry and the forest sector (FFH-Impact)" with its two sub-projects was carried out by BBW-Consultancy, Freiburg, the Forest Research Institute of Baden-Württemberg (FVA), Freiburg, the Thünen-Institute of International Forestry and Forest Economics (TI-WF), Hamburg, the Department of Forest Economics and Forest Management of the Georg-August University of Göttingen (Uni GÖ), the Faculty of Law of the University of Hamburg (Uni HH) and the Institute for Landscape Ecology and Nature Conservation (ILN), Bühl.

In the sub-project "Economic analyses for implementing the Habitats Directive in forests", conducted by BBW, TI-WF, Uni GÖ and Uni $\mathrm{HH}$, we aimed to identify and evaluate natural and economic impacts of FFH-measures on concrete forest enterprises.

In our study we focused solely on business economic impacts. A comprehensive economic analysis in terms of welfare gains and losses would also require a valuation of the benefits of the public goods arising from implementing the Habitats Directive. However, the information necessary for allowing evaluation on, e.g., the changes in species composition or the success of conserving threatened species, will not be available for a couple of years yet. Until now no public good is defined. So far the Habitats Directive only regulates processes, which results in a lack of quantity for economic valuations. Thus a comprehensive economic analysis could not be accomplished at the time of our project.

The following issues were covered in our study:

1 The determination of FFH-measures with an impact on forest management

2 The plausibility and further development of a new simulation model to assess impacts of FFH-measures over time

3 The long-term consideration of (natural and) economic impacts of FFH-measures on current and future management practices, especially the time and intensity of the occurring effects

4 The cause and effects of the impact of FFH-measures

The following chapters provide findings from the research project. Further, more detailed information on the following chapters can be found in Rosenkranz et al. (2012), Seintsch et al. (2012), and Wippel et al. (2012). Yet, as not all management plans for forest areas in the Laender are completed and-for economical reasons-only a limited amount of case studies could be evaluated, this study does not claim to be representative for all forest enterprises in Germany. It is rather to be regarded as a snapshot of the current implementation process.

\section{Literature review}

In regard to our research objective there are basically two approaches for evaluating the effects of nature protection measures: the assessment of benefits and the assessment of costs. An important means for assessing the benefits of nature protection measures is, for example, the Contingent Valuation Method (CVM) to determine the Willingness to Pay (WTP) for the conservation of habitats and species. This method was, amongst others, used to evaluate the WTP for the protection of Natura 2000 sites in Scotland (Gibson et al., 2004), and for an increase of protection areas in Finland (Li et al., 2004; Pouta et al., 2000). Studies evaluating the costs and benefits of forest conservation and/or Natura 2000 using the CVM were conducted amongst others by Kniivilä et al. (2002) in Finland and Amigues et al. (2002) in France. Further studies determining the effects of nature protection as well as recreation measures are named in Elsasser et al. (2009).

The assessment of costs of nature conservation measures can be conducted by using forest growth models to simulate changes on stand level (for Central European conditions e.g. Silva (Pretzsch et al., 2002), Waldplaner (Hansen, 2012) and BWINPro (Döbbeler et al., 2011)) or by means of economic models.

A straightforward calculation of the expenses of forest enterprises derived from protection and recreational functions of forests has been carried out by Küppers and Dieter (2008). Expenditure for protection and recreational functions were analysed based on data of the forest accountancy network of the German Federal Ministry for Nutrition, Agriculture and Consumer Protection. Reductions of earnings for forest land set-aside areas were calculated by extrapolating data from the Integrated Environmental and Economic accounts of Forests for Germany. The study aimed to give an overview of the situation of forest enterprises in Germany. It did not aim to differentiate the effects of individual protection measures or of different types of protected areas on forest enterprise level.

Expenses for forest land set-aside were also calculated by Leppänen et al. (2005) and Posavec et al. (2011). The aim of the study by Leppänen et al. (2005) was the analysis of the effects of strict forest conservation on forest enterprises and the timber market. Amongst others, the net present value of normal forest stands with an even age-class distribution and mature stands was calculated using statistical data. Posavec et al. (2011) calculated the net present value of forest stands as well as the present and future cutting values for tree species, using assortment tables, average forest product prices and data on tree species out of forest management plans. Thus, they calculated one-time compensation fees on stand level for forest enterprises experiencing a land setaside due to the implementation of the Habitats Directive. In both studies enterprise individual data was not included.

Another economic approach was chosen by Wagner and Jönsson (2001). They conducted calculations to determine the average reductions of contribution margin and additional costs per hectare, comparing the FFH-measures to a normal forest. As a data basis yield tables as well as average costs and revenues were used. The impacts of forest land set-aside as well as important FFH-measures for habitat types in Natura 2000 areas were calculated. Yet, this study, too, did not aim to use enterprise-individual data or to take individual forest structures into account.

A practical evaluation tool for forest management restrictions due to nature protection measures was developed by Möhring and Rüping (2006). With their "annuity model" they provided a useful tool to evaluate economic effects of changes in forest management. Based on yield tables, average wood revenues, felling and planting costs the reductions of earnings as a result of changes in forest management practices can be assessed (Möhring and Rüping, 2006:2). The model is suitable to calculate the effects of long-term changes, i.e. changes of rotation length and change of tree species. Yet, no forest enterprise individual data is used. Also, data tables range only up to the end of rotation periods.

For the calculation of compensation for the designation of habitat trees that can be used, for example, for negotiating nature protection contracts, Möhring et al. (2010) developed a practical excel-based evaluation tool. The evaluation is based on enterprise individual data such 
as the relinquishment of wood-use, reductions of wood-value (rot and diseases), reduction of increment, as well as costs for marking and mapping of trees. However, with this tool, other nature protection measures such as, e.g., the lengthening of rotation periods or restrictions in the choice of tree species cannot be calculated.

In summary, the effects of the single measure "forest set-aside" on the net present value have, for example, been evaluated by Posavec et al. (2011), Leppänen et al. (2005) and Küppers and Dieter (2008). Möhring et al. (2010) developed a model to assess the designation of habitat trees as well as another model to evaluate the effects of the extension of the rotation period and changes in tree species composition (Möhring and Rüping, 2006). The average effects of several FFHmeasures on the contribution margin have been calculated by Wagner and Jönsson (2001). Except for the "habitat tree model" by Möhring et al. (2010), the studies did not take enterprise individual data, forest enterprise's objectives or managerial practices into account. None of these approaches evaluated the reduction of timber felling amounts on enterprise level. Also, the natural endowment of the enterprises and its changes in time was not considered.

\section{Research and theoretical framework}

The evaluation of the impacts of implementing the Habitats Directive was conducted for the two habitat types 9110 Luzulo-Fagetum and 9130 Asperulo-Fagetum beech forests. These cover $69.5 \%$ of the forest habitat area in Germany and thus form the most important protection entity, in regard to area, amongst forest habitat types (BfN, 2012; Rosenkranz et al., 2012:16). For our study we assumed that the FFHmeasures for the entire SAC from the respective management plans are transferable to the SAC-area of the single forest enterprises. As the research framework we defined a case study based approach with an enterprise individual reference by evaluating real forest enterprises with their individual objectives, management practices and natural endowments. The remaining forest area within each enterprise and further costs and expenditures (e.g. suboptimal use of manpower) was not considered. Furthermore we implied that average (static) costs and revenues of the enterprises are also valid for the beech forest habitat type area.

The main challenges of evaluating the effects of nature conservation FFH-measures on forest management are the long production cycle in forests and the natural endowment of each individual forest holding, such as forest structures and age class distributions, which influence forest management decisions and their effects in the course of time. Against this background the effects of FFH-measures on forest enterprises can only be evaluated by modelling. Our study aimed to calculate the natural and economic impacts of all FFH-measures restricting forest enterprises. Therefore we preferred a simulation model that could calculate dynamic stand developments due to the implementation of nature protection measures and depict the differences compared to a continuation of the "Status Quo" or "Enterprise Objective" forest management, respectively (Fig. 1). We also chose the cost evaluation approach as especially the opportunity costs of wood use should be evaluated. As the analysed existing models could not accommodate our needs, one of our objectives was to develop a simulation model suitable for the above-mentioned requirements.

\section{Material and methods}

\subsection{Simulation model}

As a basis for our calculations we applied an excel-based simulation model developed by Strugholtz (2010) in the framework of a master thesis at the University of Göttingen.

The simulation model was originally developed to determine the effects of silvicultural management decisions and so to be of assistance to operational managers of forest enterprises (Strugholtz, 2010:3). It is based on enterprise individual data such as the number of trees planted per hectare, "survival functions" (i.e. the possibility of calamities), costs for planting, thinning and timber harvesting, timber sales revenues, thinning practices, rotation periods and the present and future share of tree species groups.

The model is parameterised for the four main tree species groups (spruce, pine, beech and oak). It enables the calculation and visualisation of age-classes distributions, the development of felling volumes and the "silvicultural contribution margin" as well as terminating annuities for an entire forest enterprise for a time period of up to 200 years, calculated in five-year periods. The "silvicultural contribution margin" is defined as timber revenues less harvesting, planting and thinning costs (Formula 1).

$S C M_{\mathrm{var}}=\sum_{\forall j}\left(X_{j}^{t i} \cdot P_{j}^{t i}-X_{j}^{t i} \cdot C_{j}^{h a}-C_{j}^{p l}-C_{j}^{t h}\right)$

$S C M_{v a r}=$ silvicultural contribution margin of the calculation variants "Status Quo", “Enterprise Objective" and "FFH-Regime"

$X^{t i}=$ quantity of timber felled per hectare and year

$P^{t i}=$ revenues from timber sales per hectare and year

$C^{\text {ha }}=$ harvesting costs per hectare and year

$C^{p l}=$ planting costs (one-off costs)

$C^{\text {th }}=$ thinning costs (one-off costs at age 20)

$\mathrm{j}=$ tree species group

At the beginning of the simulation the model creates an original state of the enterprise based on the individual input-data such as area

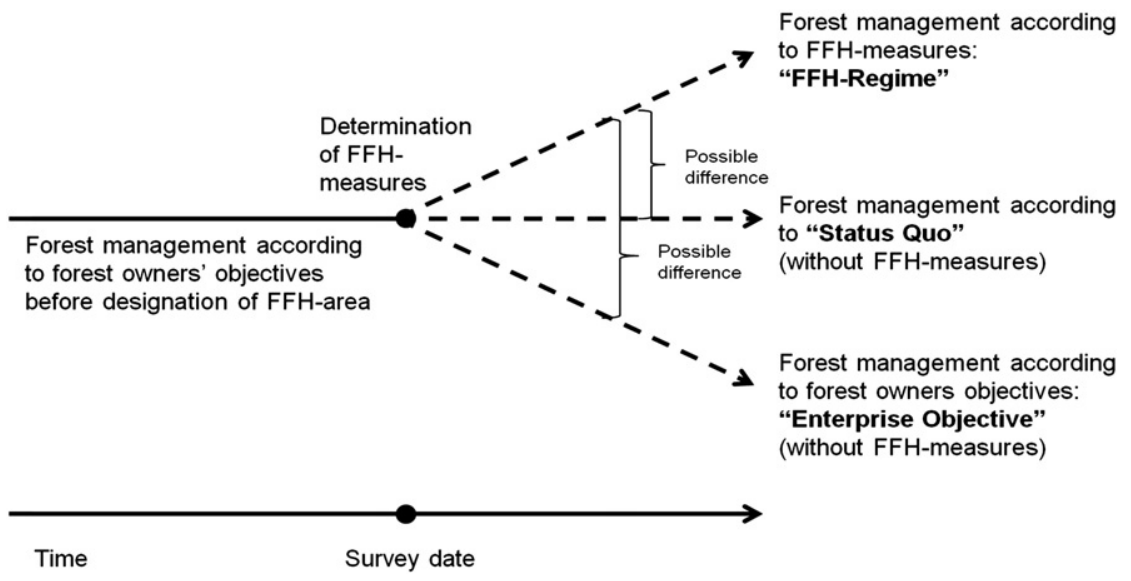

Fig. 1. Calculation concept and references of the study "FFH-Impact". 
of the tree species groups, age class distribution, stem number at planting, yield class and stocking level. The stem number for each following period is determined by a regression function and is dependent on the age class of the stands. Together with a calculated mean basal area tree (Wippermann, 2005) and the mean stand height (Smaltschinski, 1998) this number is used to determine the stand volume of each period.

The periodic increment for each tree species group is calculated from the differences between the total productivity of the 5-year calculationperiods. The underlying forest growth functions of the model are mainly based on yield tables, parameterised by Smaltschinski (1998). In a next step, timber from thinning, calamities and final felling predicated on the individual thinning practices, "survival functions" and rotation periods are calculated. Different thinning practices for each tree species group are simulated by means of adapting the stocking level. For each tree species group, current and aspired rotation periods can be calculated. After the end of the rotation period, which is simulated as a staggered felling, the model uses the given stem numbers to "plant" new stands. By determining the percentage of tree species groups of the following generation the model is able to simulate forest conversion measures (Seintsch et al., 2012).

The timber from thinning and final felling is sorted based on assortment tables by Offer and Staupendahl (2009) which are stored in the model. The timber value is determined for each period based on the enterprise specific timber prices and harvesting costs.

Table 1

Elements of the Strugholtz-Englert simulation model.

\begin{tabular}{|c|c|c|}
\hline Forest growth & Forest management & Economic calculations \\
\hline $\begin{array}{l}\text { Input data [static] } \\
\text {-Area of tree } \\
\text { species groups } \\
\text {-Age class } \\
\text { distribution } \\
\text {-Survival } \\
\text { probability } \\
\text {-Stocking level } \\
\text {-Yield class } \\
\text {-Yield functions }\end{array}$ & $\begin{array}{l}\text {-Number of trees planted } \\
\text {-Target stocking level } \\
\text { (dependent on type of thinning } \\
\text { practice) } \\
\text {-Thinning practice } \\
\text {-Type of harvesting } \\
\text {-Rotation cycle } \\
\text {-Staggered felling }\end{array}$ & $\begin{array}{l}\text {-Assortment tables } \\
\text {-Factor for reducing beech } \\
\text { timber value due to rot and } \\
\text { diseases Planting, thinning } \\
\text { and harvesting costs } \\
\text {-Timber prices for round } \\
\text { wood, industrial wood and } \\
\text { fuel wood }\end{array}$ \\
\hline $\begin{array}{l}\text { Simulation (exempla } \\
\text { Continuous } \\
\text {-Stem number } \\
\text { function } \\
\text { (based yield } \\
\text { tables) } \\
\text {-Mean basal area } \\
\text { tree } \\
\text { (based on stem } \\
\text { number, stand } \\
\text { volume and } \\
\text { mean stand } \\
\text { height) } \\
\text {-Growth } \\
\text { function } \\
\text { (based on yield } \\
\text { class and age } \\
\text { class) } \\
\text {-Increment } \\
\text { (based on stand } \\
\text { productivity and } \\
\text { time) }\end{array}$ & $\begin{array}{l}\text {-Timber from thinning (based } \\
\text { on increment, actual and } \\
\text { on target stocking level of } \\
\text { chosen thinning practice) } \\
\text {-Timber from calamities } \\
\text { (survival functions based on age } \\
\text { class and tree species specific } \\
\text { survival probabilities) } \\
\text {-Timber from final felling and } \\
\text { planting } \\
\text { (based on age class and } \\
\text { enterprise specific staggered } \\
\text { felling practice) }\end{array}$ & $\begin{array}{l}\text { Discrete } \\
\text {-Value of standing timber } \\
\text { (based on stand volume and } \\
\text { timber price function) } \\
\text {-Silvicultural contribution } \\
\text { margin } \\
\text { (based on harvested timber } \\
\text { and timber price function) } \\
\text {-Net present value } \\
\text { (based on silvicultural } \\
\text { contribution margin and } \\
\text { interest rate) } \\
\text { Annuities } \\
\text { (based on net present value) }\end{array}$ \\
\hline Management parar & $\begin{array}{l}\text { ters [dynamic] } \\
\text {-Change of tree species } \\
\text { composition } \\
\text {-Change of rotation cycle }\end{array}$ & \\
\hline $\begin{array}{l}\text { Results } \\
\text { Standing timber } \\
\text { stock }\end{array}$ & $\begin{array}{l}\text {-Raw wood supply from } \\
\text { thinning, calamities and final } \\
\text { felling } \\
\text {-Area of tree species groups } \\
\text { after thinning and final felling }\end{array}$ & $\begin{array}{l}\text {-Value of standing timber } \\
\text {-Silvicultural contribution } \\
\text { margin } \\
\text {-Net present value } \\
\text {-Annuities }\end{array}$ \\
\hline
\end{tabular}

Furthermore the annuities for each tree species group can be calculated using an enterprise individual interest rate (Strugholtz, 2010:13f.). As over a time period of 200 years no profound predictions of timber price developments can be made the model uses a static price and cost approach. Timber prices as determined by the operational managers are used throughout the entire calculation period.

As can be seen, in our study the costs for intermediate input and labour connected to the implementation of FFH-measures are already subtracted to result in the silvicultural contribution margin. Yet, a full economic accounting, in order to assess entrepreneurial income, also requires calculations of rents as well as interest paid and received (Wöhe and Döring, 2010). This is reflected in the net present values. In our study we first calculated the silvicultural contribution margins for the three calculation variants with the simulation model. In order to identify the income losses for the forest enterprises we then calculated the differences of the silvicultural contribution margins from the "FFH-Regime" to the two references "Status Quo" or "Enterprise Objective" respectively. To determine the net present values, the annual differences of silvicultural contribution margin over the 200 year simulation period were then discounted to today, using an interest rate of $1.5 \%$. In a last step we transformed the net present value of each enterprise to terminating annuities in order to show the annual differences in income for the 200 year period (Formula 2 and 3 ).

$N P V=\sum_{t=1}^{T=200} \frac{\Delta S C M}{(1+i)^{t}}$

Annuity $=N P V \cdot \frac{i(1+i)^{T}}{(1+i)^{T}-1}$

$\mathrm{NPV}=$ net present value of income losses due to FFH-measures $\triangle \mathrm{SCM}=$ differences of silvicultural contribution margins from "FFH-Regime" to the references "Status Quo" or "Enterprise Objective"

$\mathrm{T}=$ time period being evaluated (200 years)

$\mathrm{T}=$ time

$\mathrm{I}=$ interest rate

The calculation of the net present values and annuities was based only on the differences of the silvicultural contribution margins of the three calculation variants. Also, only the beech forest habitat area of the case study forest enterprises was considered. The initial and final timber stocks, as well as the forestland were not included in the calculation. The value of the initial timber stock was not regarded to be relevant for our calculations since it is equal for all three calculation variants and thus would be of no consequence when calculating differences between them. The value of the final timber stock could also be neglected due to the long discounting period. This applies also to the values of forest land beyond the 200 year period. Consequently, the simulation results were calculated using the dynamic methods of investment analysis on the basis of static economic input data.

The Strugholtz-Englert model can be classified as a dynamic and continuous model with static and discrete elements. It is a dynamic model as the simulation is conducted over a time period of 200 years. It is continuous as the state variables of the model are calculated in continuous functions and the results of the simulation process can be made visible over the entire simulation period. The presentation of the results in the model, however, is discrete, as they are aggregated in 5 year periods. Also, the economic calculations are discrete as they are based on the aggregated results of the five year periods. The static elements of the model are the input data, with the exception of the underlying assortment and yield tables, which are, in turn discrete. The dynamic elements of the simulation model are the management parameters "prolongation/ shortening of the rotation period" and the "change of tree species composition". The different elements of the model are shown in Table 1. 
In the course of our project we subjected the model to extensive plausibility tests, e.g. by means of an idealised forest enterprise, which proved satisfactory. Considerable advancements, such as the addition of a simulation for Douglas fir, a means to calculate enterprise individual staggeredfelling periods and intensities as well as the addition of a factor for the decrease in value of over-aged beech stands were accomplished by our team. In the course of the adaption process the model was renamed "Strugholtz-Englert-Model". The model was then used for the evaluations in the 21 case study forest enterprises. It was checked again for plausibility based on the results and proved to be adequate for our task.

\subsection{Calculation concept}

In our study we aimed to calculate the impacts of FFH-measures on beech forest habitat type areas. As results considerably depend on their reference the determination of references is highly significant. It can be expected, that nature conservation objectives have a major impact if they differ from enterprise and silvicultural management objectives. For a basic approach we thus compared individual forest management practices and objectives not affected to a reference forest management affected by FFH-measures. For this purpose, we decided to use two references for our case study forest enterprises.

1 The reference "Status Quo" depicts a continuation of the forest management, without restrictions from implementing the Habitats Directive, which has led to the forest condition at the time of data collection. In this manner, changes caused by FFH-measures as compared to the business as usual can be revealed. This way, the argument in political discussions that the implementation of the Habitats Directive would not lead to any changes of the current forest management, can be tested.

2 The reference "Enterprise Objective" on the other hand includes the implementation of changing objectives and silvicultural management in the future, not considering restrictions from the Habitats Directive. In this manner, changes caused by FFH-measures as compared to the intended future forest management (within the scope of legal regulations) can be revealed.

3 Furthermore the scenario "FFH-Regime" was simulated, calculating all FFH-measures regarded as a restriction for forest management of the respective enterprises.

For each forest enterprise the loss of felling amounts, of "silvicultural contribution margin" (less ongoing administrational costs for implementation of FFH-measures) and of income were calculated by comparing the results of both references to the forest management under the "FFH-Regime" (cf. Fig. 1). Also, we aimed to find out which forest enterprise characteristics in particular cause losses due to implementing the FFH-measures by a simple comparison of the arithmetic averages of losses of silvicultural contribution margin. For a better comparability of the natural and economic impacts of the FFH-measures in the case study forest enterprises the calculations were conducted only for the evaluated beech forest habitat type area. This allows an assessment of the implications of the specific FFH-measures, but implies that conclusions about the impacts of FFH-measures on the entire forest enterprises cannot be drawn. Also, as the measures for the two habitat types 9110 Luzulo-Fagetum and 9130 Asperulo-Fagetum beech forests were for the most part identical, they were examined together.

\subsection{Database}

Twenty-one private (n10), communal (n5) and state (n6) forest enterprises from the Bundeslaender Baden-Württemberg, Bavaria, Hesse, Mecklenburg West Pomerania, Lower Saxony and North Rhine Westphalia took part in the study. They were selected based on their type of ownership, their beech forest habitat area (at least 50 ha), the existence of a management plan and their willingness to reveal enterprise individual data.
At the first visit, the forest enterprises were classified by determining economical, ecological and social objectives and key figures (e.g. enterprise, forest and habitat area; contribution margins of the product sectors) by means of a partly structured interview with the operational managers of the enterprises. Current and future forest management objectives, which were needed to form the calculation references, were discussed. In a second step, data for the simulation model was gathered from forest management plans, the management accounting and the experience of the operational managers. This way, the different enterprise individual age class distribution of forest stands in the habitat types, harvesting costs, wood revenue, planting and thinning costs, silvicultural practices as well as rotation periods were determined. As harvesting costs and timber prices are subject to changes, e.g. due to calamities, a mean of the years 2007-2009 was used where possible. For calculating the annuities the interest rate of 1.5\%-following Möhring and Rüping (2006)-was used for all case study forest enterprises. In a third step the FFH-measures from the respective management plans were identified and of which in turn restrictions were deduced (e.g. designation of one habitat tree causes the loss of $100 \mathrm{~m}^{2}$ of production area). During a second visit the results of the calculations were discussed and validated with the operational managers and, where possible, also with nature protection authorities.

\subsection{Classification of results}

In order to minimise effort for data acquisition for the case study forest enterprises no comprehensive analyses of the economic impacts of FFH-measures on the entire enterprises but only evaluations for the beech forest habitat type areas were carried out. Thus, to be able to classify our results into the current situation of German forest enterprises we conducted a special evaluation of the forest accountancy network of the Federal Ministry for Nutrition, Agriculture and Consumer Protection, Germany. This nationwide forest accountancy network comprises about 350 private, communal and state forest enterprises with at least 200 ha of forest area. Annually, silvicultural and accounting data is raised in these enterprises and used for an extrapolation of the current situation of German forestry (Bormann et al., 2005; BMELV, 2012).

For the classification of our results into further costs and income of German forest enterprises we calculated the silvicultural contribution margin and the net profit (operational income incl. state aid and premiums less operational expenses and imputed costs for owners in management) of the network enterprises. To achieve comparability to our case study forest enterprises only network enterprises with a share of beech forest of more than $70 \%$ of wood ground area, which is the minimum area required for a beech habitat, were chosen for this evaluation. That way, in regard to the tree species distribution, the network enterprises approximately had "habitat character". For the years 2003-2010 data of 9-12 private and communal network enterprises was at hand. Data on age class distribution, protected areas and subsidies of the network enterprises was, however, not available. Thus the special evaluation only serves for giving an overview and a rough classification of the study results. The comparison of our results with the net profit of the network enterprises can, however, show the dimension of relative economic losses forest enterprises may experience.

\section{Results}

\subsection{Case study forest enterprises}

With the partaking forest enterprises a great diversity in enterprise structures, such as ecological and economic orientation as well as large, medium and small scale enterprises, could be covered.

The total wood ground area of the twenty-one enterprises ranged between 100 ha and 15,000 ha, summing up to 97,000 ha in total. With an average share of deciduous trees of $38 \%$ coniferous trees were clearly dominating in the case study forest enterprises. The private 


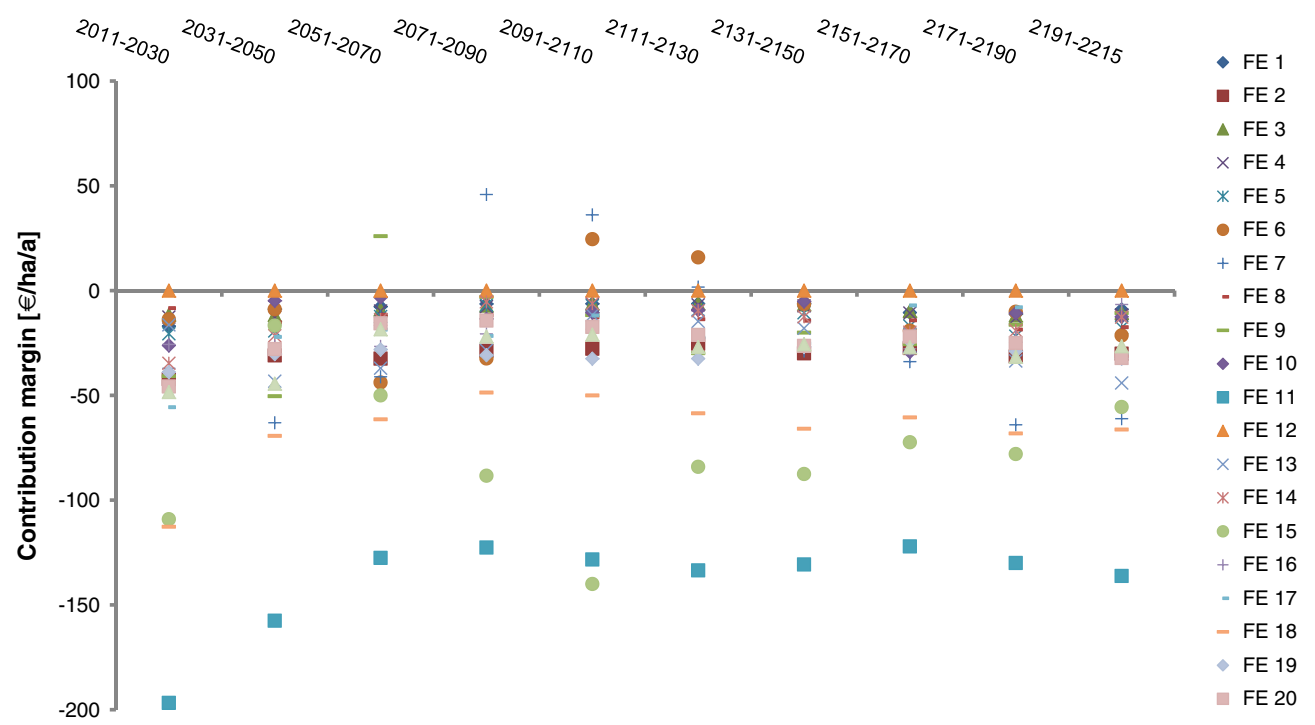

20 -year periods

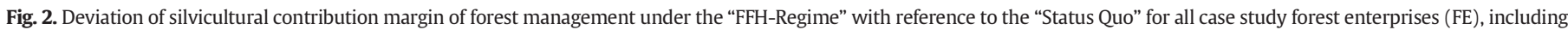
additional ongoing administrative costs.

forests featured larger shares of coniferous trees than the communal and state forest enterprises. The total felling quantity of the enterprises ranged between 3.8 and $9.8 \mathrm{~m}^{2} / \mathrm{ha} / \mathrm{a}$.

The evaluated area of beech forest habitat types ranged between about 50 and 2570 ha. Altogether, about 13,300 ha of beech forest habitat type area could be analysed. The dominating tree species on the habitat type areas was beech with a yield class between 0.8 and 2.8.

On a scale of 1 (= unimportant) to 10 (=very important) the enterprises were asked to judge their economic and ecological objectives. The economic aims were rated between 6 and 10 with an average of 8.7 over all enterprises. With a rating of 9 the economic objectives had a slightly higher significance for the private enterprises as compared to the general mean. The ecological aims were rated between 3 and 10 with an average of 7 over all case study forest enterprises. The highest significance of ecological objectives could be found in state forest enterprises with a mean of 9, and the lowest significance in private forest enterprises with an average of 5.4 .

Further information on the case study forest enterprises can be found in Appendix A (natural data) and Appendix B (economic data).

\subsection{Forest management restrictions due to FFH-measures}

Management plans contain numerous measures for beech forest habitat types. The measures most mentioned for beech forest habitat types were:

- Conservation/increase of habitat trees

- Conservation/increase of dead wood

- Conservation/increase of old-growth forest

- Conservation of habitat-specific natural species composition

o Promotion of habitat-specific native species

o Conservation of rare native tree species

o Removal of non-native tree species

- (Continuation of) Close-to-nature forestry

- Promotion of small forest structures

- Protection of soils (e.g. by use of skidding trails)

In discussion with the operational managers of the case study forest enterprises it became clear that, however, due to the existing objectives and managerial concepts and objectives, not all of these measures were regarded as a restriction to forest management. In one forest enterprise, for example, FFH-measures had no negative impact at all, as the current and future enterprise objectives and management practices were congruent with the requirements. In the other enterprises, as most restrictive measures and their effects were named:

(1) The designation of habitat trees and (islets of) old-growth trees, leading to a permanent loss of production area and an increase in expenses for work safety and for safety precautions in places of public traffic due to a higher quantity of dead wood in beech crowns.

(2) The conservation of habitat-typical woody species, restricting the choice of future tree species (e.g. more profitable tree species or the planting of stable mixed forests for climate change) and causing increasing costs for the removal of non-habitat typical natural regeneration.

(3) The conservation of a sufficient share of beech stands in the maturity stage, implicating an extension of rotation periods and a devaluation of mature timber due to red heartwood and wood decay.

Also, an increase in ongoing administrational costs, for example, due to a rise in the complexity of organisational work deriving from the implementation of FFH-measures was noted. In addition, forest enterprises named one-time administrational costs, e.g. for the process of SAC-designation, which were not included in our evaluations.

\subsection{Changes of felling quantities and silvicultural contribution margin}

The first analytical step was to compare the difference of the harvested amount of timber between the calculation scenario "FFHRegime" (all FFH-measures) to the references "Status Quo" (business as usual) and "Enterprise Objective". The average reduction of timber amounts for the "FFH-Regime" in comparison with the "Status Quo" on the beech forest habitat areas of the 21 case study forest enterprises ranges between $0-1.2 \mathrm{~m}^{3} / \mathrm{ha} / \mathrm{a}$ in the 200 year simulation period. The average loss over all enterprises is $0.4 \mathrm{~m}^{3} / \mathrm{ha} / \mathrm{a}$. Great variations of the reductions of felling amounts could be noted over the 200 year period. The span of changes of the amounts of harvested timber ranged between -2.7 and $+1.9 \mathrm{~m}^{3} / \mathrm{ha} / \mathrm{a}$. Comparing the reference "Enterprise Objective" to the forest management under the "FFH-Regime" future changes in the share of tree species groups and changes in the rotation period were taken into account. The average reduction of the amount of harvested timber of each enterprise in this comparison lies between $0-1.6 \mathrm{~m}^{3} / \mathrm{ha} / \mathrm{a}$ with an average over all enterprises of $0.66 \mathrm{~m}^{3} / \mathrm{ha} / \mathrm{a}$. 
The span of changes of the amount of harvested timber in the 200 year period even ranges between -5.3 and $+3.7 \mathrm{~m}^{3} / \mathrm{ha} / \mathrm{a}$.

In Fig. 2 the deviation of silvicultural contribution margin of forest management under the "FFH-Regime" with reference to the "Status Quo" for all case study forest enterprises is visualised. The ongoing additional and administrative costs are included. The results are depicted in 20 -year periods for the next 200 years. On first view, great losses for each forest enterprise, especially in the first three periods, become apparent. Changes in contribution margin move between -136 and $+46 € /$ ha/a in the course of the 200 year period on the enterprises' habitat areas. The average reduction of silvicultural contribution margin of the enterprises lies mainly between 0 and $120 € /$ ha/a. The average loss over all forest enterprises amounts to $29 € /$ ha/a.

The deviations of silvicultural contribution margin for the case study forest enterprises of forest management under the "FFH-Regime" with reference to the "Enterprise Objective" are shown in Fig. 3. It becomes apparent that the forest enterprises experience even higher losses of silvicultural contribution margin and greater ranges as compared to the "Status Quo". The average silvicultural contribution margin of the single enterprises is reduced between zero and $-180 € / \mathrm{ha} / \mathrm{a}$ in the course of the 200-year simulation period. The average reduction (arithmetic average) on the beech forest habitat areas over all enterprises is $40 € / \mathrm{ha} /$ a. The variation of silvicultural contribution margin in the 200 year simulation period ranges between -234 and $+97 € /$ ha/a.

6.4. Reasons for changes of felling amounts and silvicultural contribution margin in the course of time

In order to find reasons for the range and variations of the results from the case study forest enterprises further analyses, such as testing single FFH-measures for selected case study forest enterprises and additional simulations with idealised enterprises, were conducted.

We found out, that the designation of habitat trees and the extension of rotation periods have direct short-term impacts on forest enterprises whereas restrictions in the choice of tree species have a long-term effect.

Deviations of the amount of harvested timber and the silvicultural contribution margin of the "FFH-Regime" as compared to the "Status Quo" are provoked by various factors. In general, reductions are induced by the designation of habitat trees-equalling a small area of land set- aside-and the resulting loss of forest management area. A designation of habitat trees in mature stands hence implies a loss of management area in particularly valuable stands.

The great variations over the regarded 200 year simulation period, occurring in some of the enterprises, are especially due to their respective age class distribution. For enterprises with a high share of mature beech stands extending the production cycle to enhance or maintain the share of old-growth trees leads to a harvesting of these stands at a later date than planned. Thus, in the first simulation periods some forest enterprises can realise less felling amounts under the "FFH-Regime" as they would have done in a forest management after the "Status Quo". In later periods, however, a higher amount of timber as compared to the "Status Quo" has accrued due to the postponement and the still high increment rates of mature beech (suspended harvesting). Yet, it needs to be considered that, although there may be higher amounts of harvested timber a loss of wood value by wood rot and red heartwood is highly likely.

The loss of contribution margin through implementation of FFHmeasures is due to the reduction of felling amounts as specified above, to the higher costs for felling in regard to work safety measures due to dead wood in beech crowns and to further additional administrative costs. In some periods, the increased rotation lengths can shift income from final felling and thus lead to temporary higher income in later periods as compared to the "Status Quo". However, the loss of first periods can lead to liquidity problems and can also not be wholly compensated by higher incomes in later simulation periods.

Comparing the reference "Enterprise Objective" to the "FFH-Regime", requirements in choice of tree species derived from FFH-measures often intensify the abovementioned effects. Some case study forest enterprises intended to increase the share of coniferous trees in mature beech stands after final felling in order to raise the medium and long term timber yield. The restrictions lead in consequence-especially in later periods when the coniferous trees have matured-to the high differences of felling amounts and silvicultural contribution margin between the reference "Enterprise Objective" and the scenario "FFH-Regime".

\subsection{Causes and effects}

As becomes clear in Figs. 2 and 3, FFH-measures in some forest enterprises have stronger negative impacts than in others. In one enterprise,

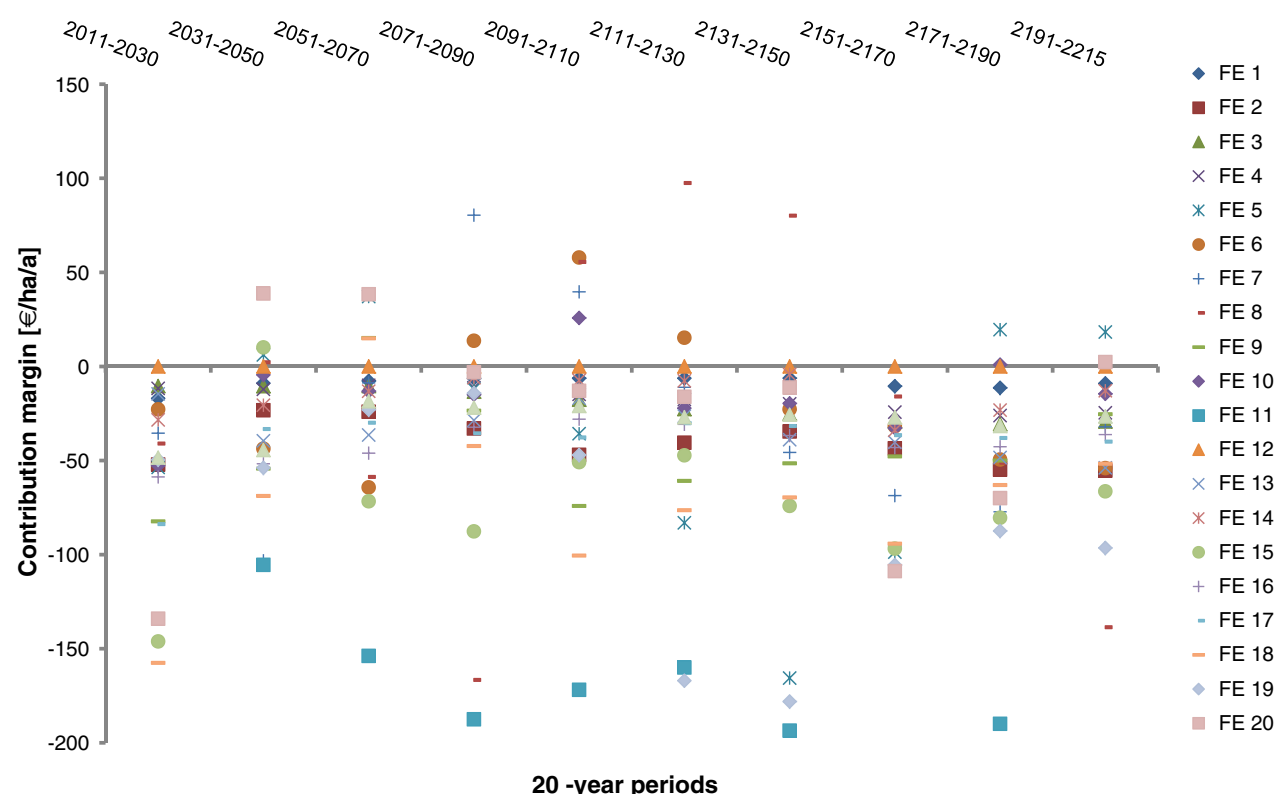

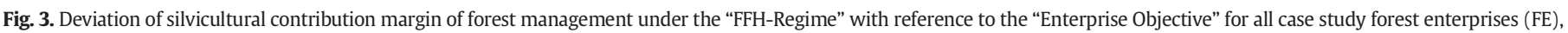
including additional ongoing administrative costs. 
income reductions of over $200 € /$ ha/a occurred in a few calculation periods. In the following, the results of the comparison of losses in contribution margin for various characteristics of forest enterprises and FFHmeasures are shown (Table 2). Due to the small number of case study forest enterprises the results of the analysis can, however, only depict rough estimations and trends.

The characteristics chosen for the analysis were (1) enterprise objectives, based on a self-assessment of the case study enterprises (importance of objectives on a scale of 1 to 10 , based on indicators), (2) the average yield class for beech on beech forest habitat areas (based on the yield table by Schober, 1975), (3) the loss of the enterprises' forestry area due to designation of habitat trees in beech forest habitat areas, (4) the share of beech stands above the rotation period and (5) the aspired share of coniferous trees in beech forest habitat areas. These factors give a "quick and easy overview" over the characteristics of forest enterprises under the "FFH-Regime" and are easy to collect.

When comparing the average silvicultural contribution margins of the "FFH-Regime" to the "Status Quo" between enterprises with different enterprise objectives, the high average losses for the parameter "ecological as high as economical objectives" come unexpected. In this case they are due to one enterprise with an above-average number of designated habitat trees and an extension of the rotation period which lowers the average of the 4 enterprises considered. Regarding the calculation scenario "FFH-Regime" to "Enterprise Objective" there is a noticeable higher reduction in silvicultural contribution margin for forest enterprises with high economic aims ( $35 € /$ ha/a) than for enterprises with equally high or higher ecologic aims.

Considering the different average yield classes of the beech forest habitat areas, the enterprises with yield classes between 1 and 1.9 experience a greater reduction of silvicultural contribution margin than enterprises with a yield class between 2 and 2.9. We assume that this is due to the designation of habitat and old-growth trees and the resulting loss of management area which likely has a stronger impact on forest stands with a high yield class.

The differences in the silvicultural contribution margin between enterprises with different shares of beech stands older than the rotation cycle show that the higher the share of over-aged beeches the higher

\section{Table 2}

Comparison of average losses of silvicultural contribution margins for exemplary forest enterprise characteristics and FFH-requirements.

\begin{tabular}{|c|c|c|c|}
\hline & $\begin{array}{l}\text { Number of } \\
\text { enterprises } \\
\text { (of 21) }\end{array}$ & $\begin{array}{l}\text { "FFH-Regime" } \\
\text { compared to "Status } \\
\text { Quo" (€/ha/year) }\end{array}$ & $\begin{array}{l}\text { "FFH-Regime" compared } \\
\text { to "Enterprise Objective" } \\
(€ / \text { ha/year) }\end{array}$ \\
\hline \multicolumn{4}{|l|}{ 1. Owner goals } \\
\hline $\begin{array}{l}\text { High economic } \\
\text { objective }\end{array}$ & 13 & -18 & -35 \\
\hline $\begin{array}{l}\text { Ecological as high } \\
\text { as economic } \\
\text { objective }\end{array}$ & 4 & -28 & -30 \\
\hline $\begin{array}{l}\text { High ecological } \\
\text { objective }\end{array}$ & 4 & -19 & -19 \\
\hline \multicolumn{4}{|l|}{ 2. Yield class } \\
\hline $0.8-1.9$ & 12 & -24 & -35 \\
\hline $2.0-2.9$ & 9 & -15 & -26 \\
\hline \multicolumn{4}{|c|}{ 3. Loss of the enterprises' forestry area due to designation of habitat } \\
\hline $0-10 \%$ & 11 & -16 & -21 \\
\hline $11-20 \%$ & 6 & -18 & -37 \\
\hline$>20 \%$ & 4 & -33 & -49 \\
\hline \multicolumn{4}{|c|}{ 4. Share of beech stands above the rotation period } \\
\hline $0-3 \%$ & 6 & -8 & -17 \\
\hline $4-6 \%$ & 10 & -17 & -33 \\
\hline $7-10 \%$ & 5 & -41 & -44 \\
\hline \multicolumn{4}{|c|}{ 6. Aspired share of coniferous trees in beech forest habitat areas } \\
\hline $0 \%$ & 10 & - & -24 \\
\hline $1-20 \%$ & 6 & - & -27 \\
\hline$>20 \%$ & 5 & - & -50 \\
\hline
\end{tabular}

are also the income reductions when implementing FFH-measures. This is on the one hand due to the designation of a great number of habitat trees especially in old stands. On the other hand the required maintenance of the share of old-growth trees can delay the timber harvest in mature stands.

In regard to the differences in silvicultural contribution margin between enterprises with different shares of management area designated for habitat trees Table 1 shows that the higher the percentage of area designated for habitat trees, the higher the loss of silvicultural contribution margin. This is caused by the reduction of managerial area but also by an increase of costs for, e.g., work safety measures.

As a last issue the impacts on enterprises aiming to plant different shares of coniferous trees in beech forest habitat areas are depicted in the table. This analysis was only conducted for the comparison "FFHRegime" to "Enterprise Objective". As a result of the high yield power of coniferous trees that would have led to an increase of timber in the future, enterprises planning to plant a bigger share of coniferous trees after final felling of beech on the habitat areas experience greater reductions of income.

\subsection{Calculation of income losses}

The income losses were evaluated by discounting the differences of the silvicultural contribution margins (including additional ongoing administrative costs) of the "Status Quo", the "Enterprise Objective" and the "FFH-Regime" of the 200 year simulation period with an interest rate of $1.5 \%$. In a next step we transformed the net present values of the differences of the silvicultural contribution margins to terminating annuities in order to show the annual differences in income (see Formula 2 and 3 ).

The average income losses of the case study enterprises comparing the "Status Quo" to the "FFH-Regime" amount to $1958 € /$ ha, ranging from 0 to $-9418 € /$ ha. The annual differences in income lay between zero and $149 € /$ ha/a with an average of $-31 € /$ ha/a.

Comparing the net present value of the "Enterprise Objective" to the "FFH-Regime" income losses between zero and 10,945 €/ha become apparent (see Appendix B). The average loss of income of all case study enterprises is $2496 € /$ ha. Here the annuities showed a range of zero to $-173 € / \mathrm{ha} / \mathrm{a}$ and an average of $-39 € /$ ha/a.

\subsection{Classification of results with the forest accountancy network}

For the classification of our results data from beech dominated enterprises from the German forest accountancy network was used. In a first step the silvicultural contribution margin of the network enterprises was calculated for the time series of 2003 to 2010. Compared to the case study forest enterprises the silvicultural contribution margins per hectare of the network enterprises showed similar results and thus verify the results from our model calculations.

Regarding the average of all network enterprises a strong increase of silvicultural contribution margin became apparent. As felling costs were almost constant during these years the rise in silvicultural contribution margin is due to an increase in timber prices.

In a next step the net profit of the network enterprises was calculated (Fig. 4). In the year 2003 private as well as communal forest enterprises were in deficit. Whereas private forest enterprises experienced a strong increase in net profit up to $150 € /$ ha in the following years, communal enterprises were in deficit in the years 2003-2007 and again in 2009. These deficits are likely originated in high costs, for example, for protection and restoration, recreation, environmental education and services for third parties. After 2009 an increase of net profit can be seen in both ownership types.

The average net profit over the evaluated private and communal network enterprises is $42 € / \mathrm{ha} / \mathrm{a}$. In comparison, the average income losses (annuities) of the case study forest enterprise on beech forest habitat type areas due to FFH-measures is $31 € /$ ha/a (Status Quo) and 


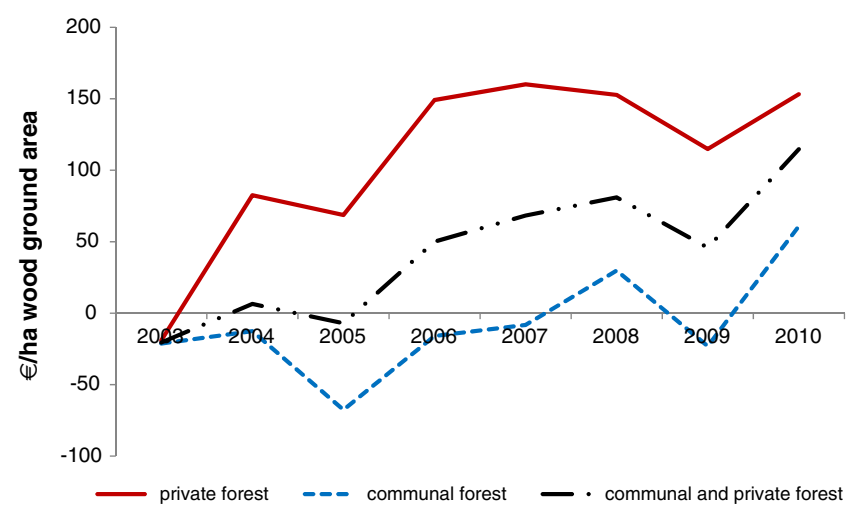

Fig. 4. Net income of forest enterprises of the test enterprise network with more than $70 \%$ beech of wood ground area.

$39 € /$ ha/a (Enterprise Objective). For the network enterprises this would mean a net profit reduction of $73 \%$ to $92 \%$ on beech forest habitat type areas. It should be considered, though, that the net profit of the private network forest enterprises differs greatly from the communal network enterprises.

\section{Discussion}

\subsection{Impacts of FFH-measures on current and future management practices}

In the course of our study we found out, that there are many FFHmeasures for beech forest habitat types. However, not all of them were felt to be restrictive by the case study forest enterprises. Apart from an increase in administrative costs on the habitat areas, mainly the designation of habitat trees, the maintenance of a share of oldgrowth stands and the protection of habitat-specific species were regarded to be a restriction to forest management. These findings could result from the predominantly qualitative and non-operational designs of FFH-measures (e.g. continuation of close-to-nature forestry), which leave room for interpretation. Moreover, it must be considered that in the face of the long production periods estimations of forest enterprises were made under the impact of the current economical framework. In view of the increasing demand for energy (e.g. Mantau, 2012) the non-use of wood residues for the maintenance of a sufficient share of dead wood could be rated differently in the future.

Our findings show, that the restricting FFH-measures can have serious impacts on forest enterprises. The number of habitat trees in mature stands and the delay of final felling have direct impact on the liquidity of forest enterprises and limit the possibility to react to market demands in the short term. In contrast, apart from possible planting costs the restrictions in the choice of tree species have a medium to long term consequence, coming into effect when felling amounts from thinning and final felling cannot be realised.

The results of our study are, of course, dependent on the reference. The restrictions felt are, for example, connected to the respective managerial objectives of the forest enterprises. Our approach in this study was to use the two references: "Status Quo" and "Enterprise Objective". By this means, impacts on both, current management as well as future changes (values of the options) could be evaluated for each individual forest enterprise. With the reference "Status Quo", the argument often used in political discussions, that the implementation of the Habitats Directive would not lead to any changes of the current forest management, could be tested. By revealing costs for forest enterprises resulting from the designation of habitat trees, the increase in work safety costs due to dead wood in crowns and by the increase of ongoing administrative costs, this argument can be refuted. The advantages of the reference "Enterprise Objectives" are on the one hand the high acceptance in the enterprises and on the other hand-e.g. in regard to compensation amounts-the assumedly lower losses as compared to a profit-oriented forest management as a reference. From our experience in the case study forest enterprises, this concept of profit-oriented forest management was not supported by any of the enterprises. The reference based on the objectives of the enterprises, however, entails that enterprises identical in costs, natural endowments and FFH-measures can be differently affected when their respective objectives are differing.

The references were also discussed with an advisory body of stakeholders from all affected interest groups and were accepted throughout all the case study forest enterprises. The underlying assumption that forest enterprise's objectives are valid over the entire simulation period of 200 years can, however, be challenged.

\subsection{The Strugholtz-Englert simulation model}

One objective of our study was the validation and further development of a simulation model to assess impacts of FFH-measures on forest enterprises in time. As a basis, the model created by Strugholtz (2010) has been chosen and adapted to project needs. The model is based on enterprise individual data that can be derived from forest management plans and business records. It is suitable to display the partially timedelayed effects of FFH-measures in the course of time. As the model is based on yield tables, calculations for forest structures and management concepts differing from age-class forests are, however, restricted. Also, costs for timber harvesting and wood prices are not dynamically calculated but are fixed over the entire simulation period, leaving room for improvement. The calculation of changes of the amount of harvested timber and silvicultural contribution margins for 200 years in advance is subject to great uncertainties. The results may be challenged as during that time management concepts, market and societal demands, site conditions or even nature protection requirements may change. We believe it important, though, to show possible future consequences of today's decisions. Due to the many different parameters, the use of the model and the interpretation of the results are challenging and timeconsuming.

\subsection{Changes of (silvicultural) contribution margin due to FFH-measures}

All in all the effects of the implementation of FFH-measures and the following losses of silvicultural contribution margin can show great variation in and amongst the case study forest enterprises when regarding different time segments of the simulation period, ranging from zero up to over $200 € /$ ha/a (Figs. 2 and 3). The losses are calculated for beech forest habitat type areas. Conclusions for the operational result of the entire enterprise can thus not be drawn, but must, amongst others, take into account the percentage of habitat area and potential further incomes, e.g. from ancillary uses.

In a related project Möhring, Stratmann and Gerst further developed the annuity model of Möhring and Rüping (2006) and applied it to standard FFH-measures (cf. Seintsch et al., 2012: 32 ff. and 91 ff.). Calculations for the loss of profit derived from the silvicultural contribution margin of the beech forest habitat type areas for ten of the case study forest enterprises were conducted with an interest rate of $1.5 \%$ for all calculations. For the designation of habitat trees an average loss of income of $35 € /$ ha/a (range: 4-59 €/ha/a) was determined. The restriction in the choice of tree species was estimated to lead to an average loss of $19 € /$ ha/a (range: $1-68 € /$ ha/a). The loss of income for the ten enterprises considering all restricting FFH-measures amounted to $66.6 € / \mathrm{ha} / \mathrm{a}$ on average. In comparison, in our study the average income losses due to FFH-measures amount to $31 € / \mathrm{ha} / \mathrm{a}$ compared to the "Status Quo" and to $39 € / \mathrm{ha} / \mathrm{a}$ compared to the "Enterprise Objective". The differences of our values compared to the annuity model most likely occur due to different methodological approaches (comparative static vs. dynamic) as well as to partly different input data. Whereas annuities for a 30 year period were calculated with the annuity model for ten enterprises we calculated annuities for 21 enterprises for the simulation period of 200 years. Furthermore Möhring, Stratmann and Gerst 
used average costs, revenues and yield tables from North Rhine Westphalia while we used enterprise individual data.

The special evaluation of the German forest accountancy network showed, that the network enterprises experienced an increase of silvicultural contribution margin due to rising timber prices while felling amounts and felling prices remained constant. In consequence, an increase in timber price will also lead to higher income losses for the implementation of FFH-measures.

\subsection{Causes and effects of the impact of FFH-measures}

The main cause for variations of costs was found to be the age class distribution. A high share of mature stands, particularly in combination with a high yield class, many habitat trees and the prolongation of the rotation cycle lead to high losses of silvicultural contribution margin. This dependency implies that equal FFH-measures have different impacts on forest enterprises with different age class structures and yield classes. Further important factors are the respective enterprise objectives, especially in regard to the desired future shares of coniferous trees in beech forest habitat types ("Enterprise Objective"). Enterprises wishing to increase the share of coniferous trees and to reduce rotation cycles were noted to have higher losses compared to the management under the "FFH-Regime". This is mostly the case for private forest enterprises and enterprises with a focus on economic objectives.

For our study we transferred the average area-specific FFH-measures on single forest enterprises. As no information on the endowment of the habitat types of the respective entire SACs was available (e.g. age class distribution), it is imaginable, that the intensity of single FFH-measures is different for the respective forest enterprises in the course of time.

\section{Conclusion}

In our study we evaluated the impacts of the implementation of FFHmeasures on forest enterprises in beech forest habitat types using an Excel-based simulation model. Differences in felling amounts, silvicultural contribution margin and annuities were calculated for 21 case study forest enterprises. Only FFH-measures that were classified as an imposition by the enterprise managers were evaluated.

As the presented results show, the determination of the impacts of FFH-measures is very complicated, as they depend, for example, on enterprise-individual natural endowments, objectives and forest management practices. Also, it became clear that income losses can vary greatly when regarding different time segments during the analysed simulation period.

Paschke and Riedinger (2012) noted that, in case of a disproportionate burden caused by the implementation of FFH-measures, forest enterprises are entitled to compensation. The following instruments for compensating income losses due to FFH-measures are currently used or being developed by the Bundeslaender in the ongoing implementation process: (1) FFH-unspecific compensation in the framework of the general existing forest subsidy schemes (e.g. forest conversion), (2) lump sum compensation payments for the compliance with all FFH-measures, (3) FFH-project specific compensation/nature conservation contracts and (4) the accounting of FFH-measures as "compensation and substitution measures" in the intervention provisions of the German Federal Nature Conservation Act ("eco-accounts").

In regard to our findings, however, average lump sum payments for compensating FFH-measures, equal for all forest enterprises or nature conservation contracts, with fixed compensation amounts may not be appropriate. The calculation of silvicultural contribution margins and losses of income, taking into account the enterprise individual conditions, as conducted in this study, could be a helpful basis for the regulation of compensation amounts.

The Strugholtz-Englert-model is much too complex to be applied in individual cases like the conclusion of specific nature conservation contracts. Nevertheless the model can be recommended for application in the process of designing compensation schemes.

In this context we suggest the compiling of compensation tables. As a first step for creating these tables the forest enterprise characteristics which were identified to affect the level of income losses, need to be classified. Model forest enterprises could then be built, covering all the combination possibilities of these characteristics by classes. In a next step the losses of income would be calculated with the simulation model for all model enterprises and all restricting FFH-measures and compiled in the table. Real forest enterprises affected by FFH-measures could then be assigned to certain model enterprises according to their individual characteristics and the losses income from the table could be applied. Since the authority for implementing the Habitat Directive lies mainly with the Laender the responsibility for drawing up such tables could also be seen with them. The Strugholtz-Englert-simulation model proved to be adequate to be applied for this task.

The loss of income as calculated in our study only equals the costs of implementing the Habitats Directive. Subject to the allocation of the property rights it could be necessary to add a premium in order to create an incentive for the voluntary engagement of forest enterprises. For Germany this is often argued since a lot of protection and recreational services traditionally are provided voluntarily by multifunctional forest enterprises (Dahm et al., 1999). It can be expected that such a premium raises the acceptance for the implementation of FFH-measures. Yet, it should be noted that rising wood demand (Mantau, 2012) and in consequence rising wood prices raise the costs of implementing the Habitat Directive und thus require price adjustment clauses in potential compensation tariffs.

Nearly no other decision in forest enterprises has as long and high an impact on economic success as the choice of tree species composition. This choice determines the point in time and the amount of revenues to cover all the costs of continuous sustainable forestry. Once a forest enterprise has taken this decision it is dependent on that path for many decades. This means that present compensation schemes of FFH-measures have, amongst others, to take into account the objectives regarding future tree species composition even though the economic effects will be due only at a much later date. It can be argued that future market and societal demands are highly uncertain and hence the effects of changing management objectives cannot be quantified in the long run. But this argument would also apply for societal decisions on forests. For example, the future societal demand for dead wood as a habitat feature is highly uncertain as well.

To sum it up, it can be said that forestry is a business subject to uncertainty and that foresters have to anticipate future circumstances based on "best guesses". This is true in particular in regard to climate change which, in order to maintain forests healthy and stable, quite likely requires a change in tree species composition.

\section{Acknowledgements}

The authors would like to thank the German Federal Ministry for Food, Agriculture and Consumer Protection (BMELV) and the Agency for Renewable Resources (FNR) for the financing and supporting of the project. We would also like to thank our project partners Prof. Dr. Dr. h.c. Gero Becker (Becker, Borchers, Wippel Consultancy Freiburg), Prof. Dr. Bernhard Möhring, Dr. Josef Stratmann and Mr. Johannes Gerst (University of Göttingen), Prof. Dr. Dr. h.c. Marian Paschke and Ms. Daniela Riedinger (University of Hamburg) for their contributions to the project. We thank Mr. André Strugholtz for the basic simulation model and assistance and Mr. Hermann Englert (Thünen-Institute of Forest Economics) for his help in enhancing the model. Special thanks go to the case study forest enterprises for providing their data and experience: without them this study would not have been possible. Last but not least we thank the anonymous reviewers for their comments and suggestions for the enhancements of this paper. 
Appendix A

\begin{tabular}{|c|c|c|c|c|c|c|c|c|c|c|c|c|c|c|c|}
\hline \multirow{2}{*}{$\begin{array}{l}\text { Forest } \\
\text { enterprise }\end{array}$} & \multirow{2}{*}{$\begin{array}{l}\text { Evaluated area } \\
\text { of beech forest } \\
\text { habitat type } \\
(9110,9130)[\text { ha] }\end{array}$} & \multirow{2}{*}{$\begin{array}{l}\text { Main } \\
\text { tree } \\
\text { species }\end{array}$} & \multirow{2}{*}{$\begin{array}{l}\text { Rotation } \\
\text { period }\end{array}$} & \multirow{2}{*}{$\begin{array}{l}\text { Yield } \\
\text { class } \\
\text { beech }\end{array}$} & \multirow{2}{*}{$\begin{array}{l}\text { Wood } \\
\text { quality }\end{array}$} & \multicolumn{10}{|c|}{ Age class distribution of beech on evaluated habitat type area [ha] } \\
\hline & & & & & & $\begin{array}{l}0-19 \\
\text { years }\end{array}$ & $\begin{array}{l}20-39 \\
\text { years }\end{array}$ & $\begin{array}{l}40-59 \\
\text { years }\end{array}$ & $\begin{array}{l}60-79 \\
\text { years }\end{array}$ & $\begin{array}{l}80-99 \\
\text { years }\end{array}$ & $\begin{array}{l}100-119 \\
\text { years }\end{array}$ & $\begin{array}{l}120-139 \\
\text { years }\end{array}$ & $\begin{array}{l}\text { 140-159 } \\
\text { years }\end{array}$ & $\begin{array}{l}160-179 \\
\text { years }\end{array}$ & $\begin{array}{l}180-200+ \\
\text { years }\end{array}$ \\
\hline FE 1 & 69.0 & Beech & 160 & 2.1 & $2^{\mathrm{a}}$ & 2.1 & 3.5 & 4.8 & 8.9 & 13.1 & 5.2 & 5.2 & 5.2 & 5.2 & 0.0 \\
\hline FE 2 & 168.0 & Beech & 140 & 1.4 & $3^{\mathrm{b}}$ & 9.8 & 12.8 & 15.9 & 17.7 & 19.6 & 6.1 & 6.1 & 6.1 & 0.0 & 0.0 \\
\hline FE 3 & 310.5 & Beech & 160 & 2.1 & 2 & 7.8 & 14.4 & 21.0 & 39.3 & 57.6 & 30.3 & 30.3 & 30.3 & 0.0 & 0.0 \\
\hline FE 4 & 200.0 & Beech & 160 & 2.1 & 2 & 5.0 & 9.2 & 13.5 & 25.2 & 36.9 & 19.4 & 19.4 & 19.4 & 0.0 & 0.0 \\
\hline FE 5 & 423.9 & Beech & 160 & 1.9 & 3 & 16.0 & 21.8 & 27.7 & 54.7 & 81.8 & 9.6 & 9.6 & 9.6 & 9.6 & 0.0 \\
\hline FE 6 & 398.8 & Beech & 160 & 1.6 & 2 & 24.6 & 20.6 & 16.7 & 16.7 & 16.7 & 55.6 & 55.6 & 55.6 & 0.0 & 0.0 \\
\hline FE 7 & 2322.0 & Beech & 140 & 1.5 & 3 & 93.3 & 127.2 & 161.0 & 318.5 & 476.1 & 56.0 & 56.0 & 56.0 & 56.0 & 0.0 \\
\hline FE 8 & 165.0 & Beech & 160 & 0.8 & 2 & 61.7 & 46.7 & 52.0 & 47.0 & 3.6 & 57.1 & 50.7 & 64.2 & 28.3 & 14.4 \\
\hline FE 9 & 514.0 & Beech & 160 & 1.7 & 2 & 22.2 & 16.9 & 72.1 & 5.9 & 0.0 & 8.3 & 4.3 & 16.5 & 0.4 & 0.0 \\
\hline FE 10 & 2322.0 & Beech & 160 & 0.8 & 2 & 85.4 & 9.3 & 58.0 & 51.9 & 49.4 & 15.9 & 45.9 & 41.1 & 4.7 & 0.0 \\
\hline FE 11 & 49.8 & Beech & 140 & 1.2 & 3 & 0.0 & 3.1 & 6.0 & 2.1 & 0.0 & 0.0 & 0.0 & 0.0 & 30.2 & 0.0 \\
\hline FE 12 & 1552.0 & Beech & 160 & 2.2 & 2 & 31.0 & 143.0 & 375.0 & 263.0 & 182.0 & 136.0 & 153.0 & 87.0 & 82.0 & 29.0 \\
\hline FE 13 & 65.5 & Beech & 160 & 2.0 & 2 & 0.0 & 0.0 & 1.4 & 3.8 & 5.0 & 29.0 & 3.9 & 3.1 & 0.0 & 0.0 \\
\hline FE 14 & 368.5 & Beech & 140 & 1.0 & $1^{\mathrm{c}}$ & 1.7 & 12.5 & 3.6 & 4.9 & 76.1 & 83.0 & 99.8 & 47.6 & 6.9 & 1.7 \\
\hline FE 15 & 146.5 & Beech & 140 & 1.1 & 2 & 0.0 & 20.8 & 31.9 & 12.4 & 13.2 & 0.0 & 19.0 & 27.3 & 0.3 & 0.3 \\
\hline FE 16 & 139.4 & Beech & 130 & 1.7 & 3 & 1.8 & 1.7 & 8.3 & 39.6 & 52.8 & 10.2 & 1.3 & 0.0 & 0.0 & 0.0 \\
\hline FE 17 & 116.3 & Beech & 130 & 2.3 & 1 & 3.1 & 24.1 & 9.7 & 7.6 & 4.9 & 6.7 & 5.3 & 8.0 & 7.6 & 0.0 \\
\hline FE 18 & 140.0 & Beech & 140 & 1.8 & 2 & 3.9 & 14.4 & 12.3 & 1.1 & 0.3 & 14.2 & 19.0 & 31.6 & 16.2 & 9.8 \\
\hline FE 19 & 2569.8 & Beech & 140 & 2.7 & 3 & 270.0 & 340.0 & 90.0 & 85.0 & 360.0 & 405.0 & 360.0 & 230.0 & 215.0 & 213.8 \\
\hline FE 20 & 245.0 & Beech & 160 & 2.8 & 2 & 1.6 & 17.5 & 0.8 & 10.0 & 6.1 & 33.9 & 0.0 & 49.0 & 43.8 & 10.0 \\
\hline FE 21 & 1030.0 & Beech & 160 & 2.7 & 2 & 6.2 & 6.9 & 21.0 & 240.9 & 34.6 & 82.7 & 93.8 & 175.2 & 64.3 & 11.6 \\
\hline
\end{tabular}

a $2=$ Average.

b $3=$ Below average.

c $1=$ Above average.

\section{Appendix B}

\begin{tabular}{|c|c|c|c|c|c|c|c|c|c|c|c|}
\hline \multirow{2}{*}{$\begin{array}{l}\text { Forest } \\
\text { enterprise }\end{array}$} & \multirow{2}{*}{$\begin{array}{l}\text { Average } \\
\text { harvesting costs } \\
\text { for beech } \\
{\left[€ / \mathrm{m}^{3}\right]}\end{array}$} & \multicolumn{3}{|c|}{ Timber prices (beech) } & \multirow{2}{*}{$\begin{array}{l}\text { Stem number at time of } \\
\text { planting }[\mathrm{N} / \mathrm{ha}]\end{array}$} & \multirow{2}{*}{$\begin{array}{l}\text { Costs for } \\
\text { planting } \\
{[€ / h a]^{a}}\end{array}$} & \multirow{2}{*}{$\begin{array}{l}\text { Costs for } \\
\text { thinning } \\
\text { [€/ha] }\end{array}$} & \multirow{2}{*}{$\begin{array}{l}\text { Interest } \\
\text { rate }\end{array}$} & \multicolumn{3}{|c|}{ Net present value } \\
\hline & & $\begin{array}{l}\text { Round wood L } 4 \\
\text { B }\left[€ / \mathrm{m}^{3}\right]\end{array}$ & $\begin{array}{l}\text { Industrial } \\
\text { wood }\left[€ / \mathrm{m}^{3}\right]\end{array}$ & $\begin{array}{l}\text { Fuel } \\
\text { wood } \\
{\left[€ / \mathrm{m}^{3}\right]}\end{array}$ & & & & & $\begin{array}{l}\text { "Status- } \\
\text { Quo" } \\
\text { (€/ha) }\end{array}$ & $\begin{array}{l}\text { "Enterprise } \\
\text { Objective" } \\
(€ / \text { ha) }\end{array}$ & $\begin{array}{l}\text { "FFH- } \\
\text { Regime" } \\
(€ / \text { ha })\end{array}$ \\
\hline FE 1 & 24 & 100 & 50 & 20 & 7000 & 1000 & 700 & $1.5 \%$ & 7489 & 7489 & 6,852 \\
\hline FE 2 & 15.7 & 85 & 50 & 15 & 5000 & 1000 & 1000 & $1.5 \%$ & 10,157 & 10,438 & 8,091 \\
\hline FE 3 & 24 & 100 & 50 & 20 & 7000 & 1000 & 700 & $1.5 \%$ & 7049 & 7333 & 6,458 \\
\hline FE 4 & 20 & 100 & 50 & 20 & 7000 & 1000 & 700 & $1.5 \%$ & 8466 & 8647 & 7,819 \\
\hline FE 5 & 15.6 & 95 & 50 & 14 & 5000 & 0 & 1000 & $1.5 \%$ & 11,736 & 12,479 & 10,806 \\
\hline FE 6 & 18 & 85 & 50 & 25 & 6000 & 0 & 200 & $1.5 \%$ & 9640 & 10,096 & 8,761 \\
\hline FE 7 & 13.7 & 85 & 50 & 21 & 8000 & 200 & 750 & $1.5 \%$ & 12,474 & 12,521 & 10,854 \\
\hline FE 8 & 15 & 80 & 42 & 20 & 7000 & 1000 & 500 & $1.5 \%$ & 7815 & 8779 & 7,069 \\
\hline FE 9 & 17 & 80 & 50 & 20 & 7000 & 1000 & 1000 & $1.5 \%$ & 10,266 & 11,848 & 8,867 \\
\hline FE 10 & 17 & 80 & 50 & 20 & 7000 & 1000 & 1000 & $1.5 \%$ & 6915 & 7346 & 6,095 \\
\hline FE 11 & 17.4 & 70 & 55 & 20 & 7500 & 200 & 400 & $1.5 \%$ & 7134 & 8661 & $-2,284$ \\
\hline FE 12 & 22.3 & $62(C)$ & 43 & 43 & 7000 & 0 & 0 & $1.5 \%$ & - & - & - \\
\hline FE 13 & 25 & 90 & 40 & 40 & 6000 & 5000 & 410 & $1.5 \%$ & 6955 & 7074 & 5,307 \\
\hline FE 14 & 22 & 95 & 52 & 26 & 8000 & 0 & 0 & $1.5 \%$ & 13,457 & 13,344 & 12,247 \\
\hline FE 15 & 15 & 100.8 & 57 & 32 & 20,000 & 0 & 350 & $1.5 \%$ & 13,045 & 12,895 & 8,361 \\
\hline FE 16 & 19 & 115.2 & 48 & 29 & 5000 & 9050 & 525 & $1.5 \%$ & 8280 & 9620 & 6,790 \\
\hline FE 17 & 17.5 & 120.96 & 48 & 29 & 5000 & 5000 & 525 & $1.5 \%$ & 11,610 & 12,731 & 9,766 \\
\hline FE 18 & 17 & 100 & 45 & 50 & 10,000 & 30 & 500 & $1.5 \%$ & 13,678 & 14,129 & 9,034 \\
\hline FE 19 & 15 & 62 & 40 & 40 & 6000 & 1600 & 0 & $1.5 \%$ & 6071 & 7714 & 4,076 \\
\hline FE 20 & 16 & 92.5 & 50 & 50 & 8000 & 0 & 600 & $1.5 \%$ & 11,908 & 12,284 & 10,186 \\
\hline FE 21 & 25 & 92.5 & 40 & 25 & 4500 & 0 & 0 & $1.5 \%$ & 10,069 & 10,069 & 7,936 \\
\hline
\end{tabular}

a If costs are very low mainly natural regeneration is practiced.

\section{References}

Amigues, J.P., Boulatoff, C., Desaigues, B., Gauthier, C., Keith, J.E., 2002. The benefits and costs of riparian analysis habitat preservation: a willingness to accept/willingness to pay contingent valuation approach. Ecol. Econ. 43, 17-31.

Bormann, K., Küppers, J.-G., Thoroe, C., 2005. Zur ökonomischen Situation von Forstbetrieben in Deutschland. BFH, Hamburg (39 Seiten, Arbeitsbericht des Instituts für Ökonomie 2005/4).

Bundesamt für Naturschutz (BfN), 2006. Ergebnisse des Arbeitskreises "Wälder" (Bewertungsschemata). http://www.bfn.de/0316_akwald.html (Last accessed: 25.01.2013).
Bundesamt für Naturschutz (BfN), 2012. Waldlebensraumtypen in Deutschland. Sonderauswertung des BfN im Auftrag des Verbundforschungsprojektes FFHImpact. (May 2012).

Bundesministerium für Ernährung, Landwirtschaft und Verbraucherschutz (BMELV), 2012. Buchführungsergebnisse Forstwirtschaft http://www.bmelv-statistik.de/de/ testbetriebsnetz/buchfuehrungsergebnisse-forstwirtschaft/ (Last accessed 13.06.2012).

Dahm, S., Elsasser, P., Englert, H., Küppers, J.-G., Thoroe, C., 1999. Belastungen der Forstbetriebe aus der Schutz- und Erholungsfunktion des Waldes. Schriftenreihe des Bundesministeriums für Ernährung, Landwirtschaft und Forsten, Reihe A: Angewandte Wissenschaft, Heft 478, Münster-Hiltrop. (75 pp.).

Döbbeler, H., Albert, M., Schmidt, M., Nagel, J., Schröder, J., 2011. BWINPro. Programm zur Bestandesanalyse und Prognose. Handbuch zur gemeinsamen Version von BWINPro 
und BWINPro-S. Version 6.3. Nordwestdeutsche Forstliche Versuchsanstalt und TU Dresden (127 pp.).

Elsasser, P., Meyerhoff, J., Montagné, C., Stenger, A., 2009. A bibliography and database on forest valuation studies from Austria, France, Germany and Switzerland - a possible base for a concerted European approach. J. For. Econ. 15, 93-108.

EUROPÄISCHE KOMMISSION (COM), 2003. Natura 2000 und der Wald. Herausforderungen und Chancen'. Auslegungsleitfaden. Europäische Kommission, GD Umwelt, Referat Natur und biologische Vielfalt Abteilung Forst- und Landwirtschaft. Amt für amtliche Veröffentlichungen der Europäischen Gemeinschaf ten, Luxemburg $120 \mathrm{~S}$.

Gibson, H., Hanley, N., Wright, R.E., 2004. An economic assessment of the costs and benefits of Natura 2000 sites in Scotland. Project Report.Scottish Government (75 pp.).

Habitats Directive, 1992. Council directive 92/43/EEC of 21 May 1992 on the conservation of natural habitats and of wild fauna and flora. OJ L 206 (22.7.1992, 7 pp.).

Hansen, J., 2012. Der WaldPlaner - Ein Entscheidungsunterstützungssystem für den Forstbetrieb. Lecture Notes in Informatics Informationstechnologie für eine nachhaltige Landbewirtschaftung.Gesellschaft für Informatik, Bonn (URL: http://subs.emis.de/LNI/ Proceedings/Proceedings194.html. Last accessed 13.03.2013).

Kniivilä, M., Ovaskainen, V., Saastamoinen, O., 2002. Costs and benefits of forest conservation: regional and local comparisons in Eastern Finland. J. For. Econ. 8 (2), $131-150$.

Küppers, J.-G., Dieter, M., 2008. Belastungen der Forstbetriebe aus der Schutz- und Erholungsfunktion des Waldes (2003 bis 2006). Arbeitsbericht des Instituts für Ökonomie der Forst- und Holzwirtschaft 2008/04, vTI, Hamburg. (29 pp.).

Leppänen, J., Linden, M., Uusivuori, J., Pajuoja, H., 2005. The private cost and timber market implications of increasing strict forest conservation in Finland. For. Policy Econ. 7 71-83.

Li, C.-Z., Kuuluvainen, J., Pouta, E., Rekola, M., Tahvonen, O., 2004. Using choice experiments to value the Natura 2000 nature conservation programs in Finland. Environ. Resour. Econ. 29, 361-374.

Mantau, U., 2012. Standorte der Holzwirtschaft - Holzrohstoffmonitoring: Energieholzverwendung in privaten Haushalten. Marktvolumen und verwendete Holzsortimente. (Hamburg 2012).

Möhring, B., Rüping, U., 2006. Bewertungskonzept für forstliche Nutzungsbeschränkungen. Schriften zur Forstökonomie, Band 32. J. D. Sauerländer's Verlag, Frankfurt am Main (35 pp.).

Möhring, Tzschupke, Oldenburg, Schulz, Schattenberg, Jacobs, Leefken, Strugholtz, 2010. Bewertungskonzept für Einzelbäume im Rahmen des freiwilligen Vertragsnaturschutzes. Erarbeitet von einer Arbeitsgruppe des Ausschusses für Betriebswirtschaft des DFWR (16 pp.).

Offer, A., Staupendahl, K.-U., 2009. Neue Bestandessortentafeln für die Waldbewertung und ihr Einsatz in der Bewertungspraxis. Forst Holz 64 (5), 16-25.

Paschke, M., Riedinger, D., 2012. Eigentumsrechtliche Bewertung der Auswirkungen der Fauna-Flora-Habitat-Richtlinie (92/43/EWG) auf private Forstbetriebe. Rechtsgutachten erstellt im Auftrag des Johann Heinrich von Thünen Institut im Rahmen des Verbundforschungsprojektes Auswirkungen von naturschutzfachlichen Anforderungen auf die Forst- und Holzwirtschaft. Arbeitsbericht des Instituts für Ökonomie der Forstund Holzwirtschaft 06/2012, vTI, Hamburg. (42 pp.).

Polley, H., 2009. Wald in Schutzgebieten - ein Überblick. Landbauforschung vTI agriculture and forestry research - Sonderheft, Band 327 75-82.

Posavec, S., Beljan, K., Lovric, M., 2011. Model of compensation payment to the owners on Natura 2000 forest sites. Glas. šum. Pokuse 44, 19-28 (Zagreb).

Pouta, E., Rekola, M., Kuuluvainen, J., Tahvonen, O., Li, C.-Z., 2000. Contingent valuation of the Natura 2000 nature conservation programme in Finland. Forestry 73 (2), 119-128.

Pretzsch, H., Biber, P., Durský, J., 2002. The single tree-based stand simulator SILVA: construction, application and evaluation. For. Ecol. Manag. 162, 3-21.

Rosenkranz, L., Wippel, B., Seintsch, S., 2012. Umsetzung der FFH-Richtlinie im Wald in den Bundesländern. Arbeitsbericht des Instituts für Ökonomie der Forst- und Holzwirtschaft 04/2012, vTI, Hamburg. (119 pp.).

Schober, R., 1975. Ertragstafeln Wichtiger Baumarten. J.D. Sauerländer's Verlag, Frankfurt am Main (166 pp.).

Seintsch, B., Rosenkranz, L., Englert, H., Dieter, M., Wippel, B., Becker, G., Stratmann, J., Gerst, J., Möhring, B., 2012. FFH-Impact: Teil 2: Auswirkungen von FFHMaßnahmenplanungen auf Forstbetriebe. Arbeitsbericht des Instituts für Ökonomie der Forst- und Holzwirtschaft 05/2012, vTI, Hamburg. (144 pp.).

Sippel, A., 2007. Forstliche Nutzung in FFH-Gebieten. Situationsanalyse und Perspektiven. Fachstudie erstellt durch die Forstliche Versuchs- und Forschungsanstalt Baden Württemberg im Auftrag der Bundesforschungsanstalt für Forst- und Holzwirtschaft. Forstliche Versuchs- und Forschungsanstalt Baden-Württemberg, Freiburg (86 pp.).

Smaltschinski, T., 1998. Regionale Waldwachstumsprognose. Schriftenreihe Freiburger Forstliche Forschung.Universitätsdruckerei Freiburg (121 pp.).

Strugholtz, A., 2010. Ein forstbetriebliches Simulationsmodell zur ökonomischen Bewertung strategischer forstlicher Produktionsentscheidungen. (Masterarbeit) Fakultät für Forstwissenschaften und Waldökologie der Georg-August-Universität, Göttingen (77 pp.).

Wagner, S., Jönsson, A., 2001. Einschränkungen der Waldbewirtschaftung durch Naturschutzauflagen am Beispiel des europäischen Schutzgebietssystems Natura 2000. Rechtsfragen und monetäre Bewertung. Gutachten im Auftrag der Arbeitsgemeinschaft Deutscher Waldbesitzerverbände e. V. (AGDW). (130 pp.).

Winkel, G., Kaphengst, T., Herbert, S., Robaey, Z., Rosenkranz, L., Sotirov, M., 2009. EU Policy Options for the Protection of European forests Against Harmful Impacts. AlbertLudwigs-University Institute of Forest and Environmental Policy, Freiburg \& Ecologic Institute, Berlin (146 pp.).

Wippel, B., Becker, G., Seintsch, B., Rosenkranz, L., Englert, H., Dieter, M., Möhring, B. Stratmann, J., Gerst, J., Paschke, M., Riedinger, D., 2012. Projekt FFH-impact: executive summary. Arbeitsbericht des Instituts für Ökonomie der Forst- und Holzwirtschaft 03/2012, vTI, Hamburg. (29 pp.).

Wippermann, C., 2005. Ökonomische Optimierung von Durchforstungen und Umtriebszeit Eine modellgestützte Analyse am Beispiel der Kiefer. Schriften zur Forstökonomie, Band 30. J.D. Sauerländer's Verlag, Frankfurt am Main (131 pp.).

Wöhe, G., Döring, U., 2010. Einführung in die Allgemeine Betriebswirtschaftslehre, 24 Aufl. Verlag Franz Vahlen, München. 

2. ROSENKRANZ L UND SEINTSCH B (2015): Opportunitätskostenanalyse zur Implementierung des naturschutzorientierten Waldbehandlungskonzepts "Neue Multifunktionalität". Landbauforschung Appl Agric Forestry Res 65(3/4):145-160 


\section{Opportunitätskostenanalyse zur Implemen- tierung des naturschutzorientierten Waldbe- handlungskonzepts „Neue Multifunktionalität"}

Lydia Rosenkranz und Björn Seintsch*

\section{Zusammenfassung}

Zur Anhebung des Waldnaturschutzniveaus wurde von Höltermann (2013) eine Segregation von Waldfunktionen gefordert. Im Rahmen dieser "neuen Multifunktionalität" (Höltermann, 2013) soll die Waldbewirtschaftung auf drei verschiedenen Waldflächenkategorien stattfinden:

(1) Wirtschaftswald mit naturschutzfachlichen Mindeststandards,

(2) Wald mit Naturschutzvorrangfunktion und

(3) Wald ohne forstliche Bewirtschaftung.

Durch die Umsetzung naturschutzfachlicher Maßnahmen im Wald sind Mindererträge und Mehraufwendungen in der Rohholzerzeugung für Waldbesitzer zu erwarten.

In der vorliegenden Studie werden die Opportunitätskosten in der Rohholzerzeugung bei einer Umsetzung der "Neuen Multifunktionalität" mit einem forstlichen Simulationsmodell über 200 Jahre berechnet und mit einer Waldbehandlungsvariante zur Fortführung der derzeitigen Waldbewirtschaftung verglichen. Bei einer Umsetzung der „Neuen Multifunktionalität" ergeben sich aus den Modellierungsergebnissen Einschlagseinbußen von rund 11,2 Mio. Erntefestmeter/Jahr und Verzichtskosten in der forstlichen Nutzung von rund 1,0 Mrd. €/Jahr. Die Umsetzung der "Neuen Multifunktionalität" wäre nur dann eine rationale Entscheidung, wenn der zusätzliche Nutzen an Naturschutzleistungen zumindest in Höhe der Opportunitätskosten der eingeschränkten Rohholzproduktion, wenn nicht sogar in Höhe der verminderten Wertschöpfung der nachgelagerten Holzindustrie, liegen würde.

Schlagwörter: naturschutzfachliche Maßnahmen, Opportunitätskostenanalyse, Deckungsbeitrag, Forstwirtschaft

\author{
Abstract \\ Opportunity cost analysis of the nature \\ conservation-oriented silvicultural \\ concept „new multifunctionality"
}

In the context of the implementation of a higher level of nature conservation in forests, a segregation of forest functions, so called "new multifunctionality", has been suggested recently (Höltermann, 2013). This "new multifunctionality" suggests forest management in three separate management classes:

(1) productive commercial forests with minimum standards of nature conservation,

(2) forests with nature protection as the priority function and

(3) forests without forest management.

However, additional expenses and losses of income for forest owners can be expected through the implementation of nature protection measures in forests.

Therefore, this study examines the opportunity costs for timber production of the implementation of the nature conservation-oriented silvicultural concept "new multifunctionality". The evaluation was conducted for a 200-year simulation period, by means of an simulation model, and compared to a modelling of current silvicultural practices. Modelling results for the implementation of the "new multifunctionality" show losses of wood harvest of about 11.2 Mio. $\mathrm{m}^{3} /$ year and costs for the relinquishment of forest utilization of roughly one billion $€ /$ year. The implementation of the "new multifunctionality" would only be a rational decision, if its additional benefit at least equaled the economically important opportunity costs of this restricted forest utilization, if not the loss of value added of the downstream wood industry.

Keywords: nature protection measures, opportunity cost analysis, contribution margin, forestry 


\section{Problemstellung}

Von der Gesellschaft werden wachsende und teilweise konkurrierende Ansprüche an den deutschen Wald zur Verwirklichung naturschutz-, klimaschutz-, energie-, wirtschaftspolitischer und weiterer Ziele gestellt. In Folge dieser Entwicklung haben die Nutzungskonkurrenzen um Waldflächen- und Rohholznutzung zugenommen. Besonders die gesellschaftlichen Ansprüche an den Waldnaturschutz sind gestiegen. Diese Waldnaturschutzansprüche werden von der Politik aufgegriffen und sind in der Biodiversitätsstrategie der Bundesregierung als Ziele zur Erhöhung des Waldnaturschutzniveaus formuliert (BMU, 2007). Unter Umständen werden in Zukunft nicht sämtliche heutigen Ansprüche an Wald- und Rohholznutzung in vollem Umfang erfüllbar sein (Dieter et al., 2008). Vor diesem Hintergrund erlangen Politikfolgenabschätzungen zu den gesellschaftlichen Waldansprüchen und deren Implementierung an Bedeutung.

Das forstpolitische Leitbild zur Bereitstellung der unterschiedlichen Waldfunktionen in Deutschland war bisher der integrative Ansatz einer multifunktionalen Waldbewirtschaftung. Obwohl in der Realität aufgrund von Eigentümerzielen, standörtlichen Bedingungen, Schutzgebietskategorien und weiteren Einflussfaktoren auch unter diesem Leitbild Bewirtschaftungsunterschiede bestehen, wurde hierbei angestrebt, Nutz-, Schutz- und Erholungsfunktionen auf der gleichen Fläche zu berücksichtigen.

Im Zuge der Forderungen nach einem höheren Waldnaturschutzniveau sind Tendenzen zur flächigen Segregation von Waldfunktionen in Deutschland zu beobachten ( $z$. B. Forest Europe, 2011). Da eine Realisierung aller gesellschaftlichen Naturschutzansprüche auf multifunktional bewirtschafteten Waldflächen nicht möglich erscheint, wird von Höltermann (2013) eine Segregation der Waldfunktionen auf Teilflächen als "Neue Multifunktionalität" (NMF) zur Anhebung des Waldnaturschutzniveaus gefordert. Ziel dieser naturschutzorientierten Bewirtschaftungskonzeption für den deutschen Gesamtwald ist „[...] die Integration von Wäldern mit natürlicher Waldentwicklung in das Gesamtkonzept eines flächendeckenden, aber differenzierten Biodiversitätsmanagements mit abgestuften forstlichen Nutzungsintensitäten[..]" (Höltermann, 2013). Von Höltermann (2013) werden zur Implementierung der "Neuen Multifunktionalität" drei Waldflächenkategorien mit unterschiedlichen naturschutzorientierten Bewirtschaftungskonzepten als erforderlich erachtet:

1. Wirtschaftswald mit naturschutzfachlichen Mindeststandards

2. Wald mit Naturschutzvorrangfunktion

3. Wald ohne forstliche Bewirtschaftung

Eine Umsetzung des NMF-Konzepts würde einen Paradigmenwechsel vom bisherigen forstpolitischen Leitbild mit einem einheitlichen Bewirtschaftungskonzept "multifunktionaler Wald" hin zu einer naturschutzorientierten Segregation in Waldflächen mit unterschiedlichen Bewirtschaftungskonzepten bedeuten. Für Folgeabschätzungen zur Umsetzung des NMF-Konzepts ist jedoch festzustellen, dass dieses hinsichtlich der Bewirtschaftungsziele und -flächen noch wenig operational ist. Für die Bewirtschaftung der NMFWaldflächenkategorie "Wirtschaftswald mit naturschutzfachlichen Mindeststandards" wird von Höltermann (2013) bspw. auf die "Gute fachliche Praxis" (GfP) von Winkel und Volz (2003) verwiesen. Nach §14(2) des Bundesnaturschutzgesetzes (BNatSchG) (2009), widerspricht die forstliche Bodennutzung nicht den Zielen des Naturschutzes und der Landschaftspflege, solange sie §5(2-4) BNatSchG sowie die sich aus dem Forstrecht ergebenden Regeln beachtet. Die GfP bietet als unbestimmter Rechtsbegriff hierdurch keine quantitativen, operationalen Bewirtschaftungszielgrößen. Winkel und Volz (2003:15) stellen fest, dass unbestimmt bleibt "[...] welcher naturschutzfachliche Anspruch mit der Guten fachlichen Praxis formuliert wird [...]" und dass sich auch aus "[...] dem forstlichen Fachrecht [...] eine solche Konkretisierung nicht ergibt [...]". Dementsprechend konnte über die von Winkel und Volz (2003) erarbeiteten Kriterien kein Konsens hergestellt werden. Vor diesem Hintergrund lassen sich daher zur Stellung der GfP bei einer Implementierung der "Neuen Multifunktionalität" keine Annahme treffen. Die für eine Folgenabschätzung erforderliche Operationalisierung des NMF-Konzeptes von Höltermann (2013) kann daher nur auf Basis zum Teil pauschaler politischer Zielstellungen getroffen werden (z. B. Biodiversitätsstrategie (BMU, 2007) oder Waldstrategie 2020 (BMELV, 2011)). Für die NMFWaldflächenkategorie "Wirtschaftswald mit naturschutzfachlichen Mindeststandards" wird eine Bewirtschaftung nach hochwertigen ökologischen Standards im Rahmen einer freiwilligen Selbstverpflichtung unterstellt.

Bislang wurden in der öffentlichen Diskussion um die Forderung nach einer Erhöhung des Waldnaturschutzniveaus häufig nur die Umsetzung einzelner naturschutzfachlicher Maßnahmen und deren Wirkungen auf andere Waldfunktionen isoliert betrachtet, jedoch nicht die Wirkung eines Naturschutzgesamtkonzeptes auf die Waldwirtschaft. Durch die Umsetzung naturschutzfachlicher Maßnahmen auf Waldflächen sind Mehraufwendungen für Waldeigentümer in der forstlichen Nutzung bspw. durch eine Erhöhung von Holzernte- und Verwaltungskosten oder durch zusätzliche Kosten für spezielle Naturschutzmaßnahmen, z. B. für Biotoppflege, zu erwarten. Zahlreiche Naturschutzmaßnahmen zur Erhöhung des Waldnaturschutzniveaus werden jedoch durch die Eigentümer nicht durch aktive Leistungserstellung erbracht und sind als Aufwendungen messbar, sondern zielen auf eine Einstellung oder Extensivierung der forstlichen Nutzung (Rohholzproduktion). Solche Maßnahmen wirken als Mindererträge (Opportunitätskosten), welche entgangene oder nicht realisierte Erträge der forstlichen Nutzung durch Unterlassungen darstellen und nicht auf Basis forstbetrieblicher Buchführungsergebnisse ermittelbar sind (Seintsch et al., 2012).

Mindererträge der Forstwirtschaft durch Einstellung der forstlichen Nutzung wurden von Küppers und Dieter (2008) auf Basis der Waldgesamtrechnung (WGR) für die Waldflächenkategorie NAWS (not available for wood supply) bewertet. Mindererträge durch Extensivierung der forstlichen Nutzung wurden unter anderem von Wagner und Jönsson (2001), Möhring und Rüping (2006), Posavec et al. (2011), 
Schmack et al. (2013) und Rosenkranz et al. (2014) ökonomisch bewertet. Von Wagner und Jönsson (2001) wurden die Deckungsbeitragsdifferenzen bei einer Umsetzung von naturschutzfachlichen Maßnahmen in FFH-Gebieten, auf Basis von Ertragstafelwerten und durchschnittlichen Erlösund Kostensätzen, abgeschätzt. Möhring und Rüping (2006) entwickelten für die Ausweisung von Mindererträgen ein Bewertungskonzept für forstliche Nutzungsbeschränkungen, in dem Ertragsverluste auf Bestandesebene, als Folge von Abweichungen des jährlichen Holzproduktionswertes von dem der forstüblichen Waldbewirtschaftung, zusammengestellt sind. Von Posavec et al. (2011) wurden Minderertragsbewertungen mit Sortierungstafeln, Forsteinrichtungsdaten sowie durchschnittlichen Holzpreisen durchgeführt. Von Rosenkranz et al. (2014) wurden die Einschlags- und Deckungsbeitragsdifferenzen für FFH-Maßnahmenbündel auf Forstbetriebsebene mit einem forstbetrieblichen Simulationsmodell berechnet. Schmack et al. (2013) modellierten Nutzungseinschränkungen für Waldbehandlungsszenarien auf Grundlage der Bundeswaldinventur BWI 2002 und FFHMaßnahmenplanungen.

Vor diesem Hintergrund ist es Ziel dieser Studie, die Opportunitätskosten (Verzichtskosten) der forstlichen Nutzung (Rohholzproduktion) bei einer Umsetzung des NMFKonzeptes von Höltermann (2013) zur Erhöhung des Waldnaturschutzniveaus für den deutschen Wald abzuschätzen. Hierfür soll das NMF-Konzept auf Basis von Literaturrecherchen und eigenen Annahmen für die Berechnung der Verzichtskosten mit einem forstlichen Simulationsmodell für die drei Waldflächenkategorien mit ihren spezifischen Bewirtschaftungskonzepten operationalisiert werden. Für den deutschen Wald soll hierbei die Differenz zwischen einer Waldbehandlung nach dem Status quo mit dem aktuellen Waldnaturschutzniveau und einer Waldbehandlung nach NMF-Implementierung mit höheren naturschutzfachlichen Standards bestimmt werden.

\section{Material und Methoden}

Dem eigenen Ansatz liegt grundsätzlich das Verständnis zugrunde, dass Naturschutz auch eine Bewirtschaftung von Wald darstellt, bei der die knappen Produktionsfaktoren Boden, Arbeit und Kapital (= Kosten) zur Bereitstellung von Gütern (= Nutzen) eingesetzt werden. Da Naturschutzgüter überwiegend öffentliche Güter sind, deren Nutzen schwierig zu bewerten bzw. unbekannt ist (d. h. es bestehen Nutzenbewertungsprobleme), wird mit dem eigenen Ansatz keine Kosten-Nutzen-Analyse, sondern eine Opportunitätskostenanalyse verfolgt.

\subsection{Das Strugholtz-Englert Simulationsmodell}

Aufgrund „[...] der langen Lebenszyklen von Waldbäumen [liegen] zwischen einer waldbaulichen Entscheidung, ihrer Realisierung und der möglichen Beobachtung und Begutachtung von allen Handlungsfolgen viele Jahrzehnte bis hin zu Jahrhunderten [...] [und eine direkte Konfrontation eines heute tätigen Bewirtschafters mit allen Folgen seiner Entscheidung [ist] [...] zumeist nur auf Grundlage von [...] Modellüberlegungen möglich" (Oesten und Roeder, 2002: 140). Zur Berechnung der Opportunitätskosten wurde daher das Strugholtz-Englert-Simulationsmodell verwendet (Strugholtz 2010; Rosenkranz et al., 2014). Mit diesem Modell können die Auswirkungen forstlicher Handlungsoptionen für einen Zeitraum von bis zu 200 Jahren berechnet werden (Strugholtz, 2010). Das Modell basiert auf Setzungen zu forstlichen Steuerungsgrößen wie u. a. Durchforstungsart, Altersstruktur, Fläche und Bestockungsgrad, Pflanzzahlen, Kultur-, Läuterungs- und Holzerntekosten und Holzerlösen, für die Holzartengruppen Buche, Eiche, Fichte und Kiefer. Zusätzlich können die zukünftigen Anteile der Hauptbaumarten in den Folgebeständen sowie Produktionszeitveränderungen angegeben werden. Der Simulationslauf ist in eine waldwachstumskundliche Modellierung und eine Modellierung des forstlichen Nutzungsverhaltens aufgeteilt. Die waldwachstumskundliche Modellierung wird auf Basis der naturalen Eingangsdaten und den ertragstafelbasierten, parametrisierten Waldwachstumsfunktionen von Smaltschinski (1998) durchgeführt. Bei der Modellierung des forstlichen Nutzungsverhaltens werden Holzmengen aus Vornutzungen, Kalamitäten und Endnutzungen im Zeitverlauf berechnet. Die Grundlage für diese Berechnung bilden u. a. betriebsindividuelle Durchforstungsvarianten, Produktionszeiten und Verjüngungsgänge für die Holzartengruppen. Nach der simulierten Endnutzung werden anhand holzartengruppenspezifischer Pflanzzahlen und der zukünftig geplanten Baumartenverteilung die Flächen neu bestockt und somit wieder in die Nutzung gebracht.

Für die Berechnung der Umsetzung des NMF-Konzeptes wurden Zustands- und Zielgrößen in den verschiedenen Bewirtschaftungskategorien im Modell teilweise variiert. Im Einzelnen betraf dies die Zustandsgröße Waldfläche sowie die Zielgrößen zukünftige Umtriebszeiten und zukünftige Baumartenzusammensetzung. Alle anderen Eingangsdaten wurden in den verschiedenen Berechnungsvarianten nicht verändert.

Mit dem Simulationsmodell wurden die folgenden Kennzahlen der Waldbewirtschaftung berechnet:

1. Holznutzungsmenge in Erntefestmeter (Efm): Summe aus Vor- und Endnutzung sowie Kalamitätsholz

2. Deckungsbeitrag: holzerntekostenfreier Erlös abzüglich Kultur- und Läuterungskosten.

Die Kennzahlen wurden jeweils für die beiden Waldbehandlungsvarianten "Neue Multifunktionalität" und "Status quo" berechnet. Anschließend wurde die Differenz aus beiden Varianten gebildet.

Da das Strugholtz-Englert-Modell bisher kein Modul für die Berechnung der Nachliefermengen für den Aufbau oder Erhalt eines definierten Totholzvorrates aufweist, wurde hierfür in einer separaten Berechnung auf den online verfügbaren Totholzkalkulator der Nordwestdeutschen Forstlichen Versuchsanstalt zurückgegriffen (Meyer et al., 2009). Das Modell berechnet den Abbau von Totholzvolumen (stehend und liegend) mit einem linearen Modell (Meyer et al., 2009). Nach diesem Modell verbleiben bei einem unterstellten 
Ausgangsvorrat von $15 \mathrm{~m}^{3}$ Totholz/ha je Baumart (stehend und liegend) nach 20 Jahren noch $3,4 \mathrm{~m}^{3}$ Totholz/ha in der Buche, 9,3 $\mathrm{m}^{3}$ Totholz/ha in der Eiche, 7,1 $\mathrm{m}^{3}$ Totholz/ha in der Fichte und 5,4 $\mathrm{m}^{3}$ Totholz/ha in der Kiefer. ${ }^{1}$

\subsection{Ertragswerte und Annuitäten}

Auf Basis der mit dem Strugholtz-Englert-Simulationsmodell berechneten Deckungsbeiträge wurden für beide Waldbehandlungsvarianten die Ertragswerte als Summe der diskontierten Barwerte zuzüglich des diskontierten Abtriebswerts der jeweils letzten Simulationsperiode sowie die Annuitäten daraus berechnet.

Für die Berechnung veränderter Ertragswerte bei einer NMF-Umsetzung wurden für den 200-jährigen Simulationszeitraum zunächst die periodischen Überschüsse der beiden Waldbehandlungsvarianten "Status quo" und "Neue Multifunktionalität" gebildet. Aus diesen periodischen Überschüssen wurde für jede Periode die Summe der Barwerte durch Diskontierung der Deckungsbeiträge pro Jahr auf den heutigen Zeitpunkt berechnet. Als Maß für die Wertänderung der Waldbestände wurden zusätzlich die Abtriebswerte zum Ende des jeweiligen Betrachtungszeitraumes (Liquidationswert: Bewertung des stehenden Holzvorrates mit dem holzerntekostenfreien Erlös pro Holzartengruppe) pro Jahr auf den heutigen Zeitpunkt diskontiert. Anschließend wurde der Ertragswert für beide Waldbehandlungsvarianten als Summe aus Barwerten und diskontierten Abtriebswerten berechnet. Die Änderung des Ertragswertes durch Umsetzung naturschutzfachlicher Maßnahmen konnte dann aus der Differenz der Ertragswerte der beiden Berechnungsvarianten für verschiedene Zeiträume innerhalb des 200-jährigen Simulationszeitraums berechnet werden. Für die Berechnungen wurde ein Zinssatz von 1,5\% unterstellt. Die Abtriebswerte auf den Stillegungsflächen gingen nicht in die Berechnung ein, da diese nicht mehr als Wirtschaftsgut für die Holzproduktion zur Verfügung stehen.

\subsection{Operationalisierung der Waldbewirtschaftungs- varianten}

Für die Berechnung der durch die Implementierung höherer naturschutzfachlicher Standards für die Forstwirtschaft entstehenden Mehraufwendungen und Mindererträge wurden die Differenzen zwischen zwei Waldbehandlungsvarianten berechnet. Die Waldbehandlungsvariante "Status quo" beinhaltet die heutige Waldbewirtschaftung mit dem aktuellen Waldnaturschutzniveau. Bei der Variante "Neue Multifunktionalität" wurde das NMF-Konzept von Höltermann (2013) durch eine Erweiterung der "Waldflächen ohne forstliche Nutzung" und von "Wald mit Naturschutzvorrangfunktion" sowie einer Umsetzung von zusätzlichen naturschutzfachlichen Maßnahmen auf der restlichen Wirtschaftswaldfläche implementiert.
Für die Umsetzung der Waldbewirtschaftungsvarianten im Simulationsmodell sind sowohl die Setzung der Eingangsdaten und Steuerungsgrößen für das Grundmodell sowie eine Operationalisierung der naturschutzfachlichen Maßnahmen nötig. Hierfür wurde im Wesentlichen auf Daten der BWI 2012, auf Vorgaben der Waldbaurichtlinien der Bundesländer ${ }^{2}$, auf die Biodiversitätsstrategie (BMU, 2007) und die Waldstrategie 2020 (BMELV, 2011), auf Zertifizierungsrichtlinien (z. B. FSC, PEFC) und die Operationalisierung von FFHMaßnahmenplanungen in Buchenwald-Lebensraumtypen (Rosenkranz et al., 2014) zurückgegriffen. Weiterhin wurden Datenbanken der im Institut vorliegenden Buchführungsergebnisse des Testbetriebsnetzes Forst des BMEL (TBN-Forst) und der Steuerungsgrößen des Basisszenarios der Waldentwicklungs- und Holzaufkommensmodellierung (WEHAM) (BMELV, 2005a) verwendet.

Als Ausgangspunkt für die Simulation der Waldbehandlungsvarianten wurden die aktuellen Ergebnisse der BWI 2012 zum Zustand des deutschen Waldes als naturale Eingangsdaten verwendet. Hierbei wurde die Fläche der Holzartengruppen Fichte (inkl. Tanne und Douglasie), Kiefer (inkl. Lärche), Buche (inkl. andere Laubbäume mit niedriger und hoher Lebensdauer, außer Eiche) und Eiche direkt aus der BWI 2012 für die bestockte, begehbare Holzbodenfläche, abzüglich Lücken und Blößen (= 10.627.513 ha), entnommen. Da die Flächen der Holzartengruppen in der BWI zum Zeitpunkt der Kalkulation nur zusammengefasst in 20-jährigen Altersklassen vorlagen, wurden diese zu gleichen Teilen auf jeweils vier 5-Jahresperioden des Simulationsmodells aufgeteilt. Eine Ausnahme bildete die letzte BWI-Altersklasse (Bäume alter als 160 Jahre). Diese wurde auf acht 5-JahresAltersklassen im Simulationsmodell aufgeteilt. In einem nächsten Schritt wurden die Steuerungsgrößen zur (naturalen) Waldbehandlung im Durchschnitt für die gesamte deutsche Waldfläche definiert. Zum Zeitpunkt der Berechnungen lag noch kein aktuelles WEHAM-Basisszenario 2012 vor, weshalb die Steuerungsgrößen zur Waldbewirtschaftung in Anlehnung an das WEHAM-Basisszenarios 2002 festgelegt (BMELV, 2005a) und um eigene Annahmen ergänzt wurden. Für alle Baumarten wurde hierbei eine starke Durchforstung sowie eine gestaffelte Endnutzung über einen Zeitraum von 20 Jahren unterstellt.

Ausgehend von diesen Grundannahmen zur forstwirtschaftlichen Waldbehandlung wurden der Status quo mit dem aktuellen Waldnaturschutzniveau sowie die zusätzlichen naturschutzfachlichen Maßnahmen zur NMF-Implementierung in forstwirtschaftliche Steuerungsgrößen mit Mehraufwands- und Minderertragstatbeständen operationalisiert (siehe Kap. 2.3.1 bis 2.3.3). Im nächsten Schritt erfolgte die ökonomische Bewertung mit holzartengruppendifferenzierten, durchschnittlichen, konstanten Preisen und Kosten über alle Waldeigentumsarten auf Basis der

\footnotetext{
Landesforst Mecklenburg-Vorpommern, 1996 und 2002; HessenForst, 2008 und 2011; SaarForst, 2003; Forst Brandenburg, 2004; BaySF, 2009; Schmalfuß et al., 2010; Niedersächsische Landesforsten, 2011; Landesforsten Rheinland-Pfalz, 2011; Landesforstbetrieb Sachsen Anhalt, 2014; Thüringen-Forst, ohne Jahr
} 
Buchführungsergebnisse der TBN-Forstbetriebe (z. B. Holzerlöse, Kultur-, Läuterungs- und Holzerntekosten). Hierfür wurde auf die TBN-Daten des Jahres 2011 zurückgegriffen. Das Jahr 2011 wurde gewählt, da in diesem Jahr kein größeres, preisbeeinflussendes Sturmereignis stattgefunden und sich die Holzerlöse nach der Weltwirtschaftskrise wieder stabilisiert hatten (vgl. Ermisch et al., 2013).

\subsubsection{Wald ohne forstliche Bewirtschaftung}

Als Ausgangsfläche unserer Berechnung wurde der aktuell bestockte, begehbare Holzboden abzüglich Lücken und Blößen von 10.627.513 ha (BMEL, 2012) unterstellt. Die aktuelle "Waldfläche ohne forstliche Bewirtschaftung" wird durch das NWE5-Projekt mit 213.145 ha angegeben. Im Rahmen des NWE5-Projektes wurden ausschließlich Waldflächen mit "natürlicher Waldentwicklung" erfasst, welche eine Mindestflächengröße von 0,3 ha aufweisen, dauerhaft nicht forstlich genutzt werden (ohne zeitliche Begrenzung) und deren dauerhafter Schutzstatus durch rechtliche oder vertragliche Sicherstellung oder dokumentierte Eigenbindung nachgewiesen ist (Engel, 2013: 9). Die BWI 2012 weist dagegen eine Fläche von ca. 450.000 ha Holzboden aus, „[...] auf der Holznutzung nicht erlaubt oder nicht zu erwarten ist [...]" (BMEL, 2012: 38). Hierin sind jedoch auch bspw. Waldflächen enthalten, bei denen eine Einstellung der Holznutzung nicht durch einen dauerhaften und formalen Schutzstatus geregelt ist. Daher wird in unserer Studie zur Definition des Status quo auf die Ergebnisse des NWE5-Projektes (213.145 ha Wald mit natürlicher Waldentwicklung) zurückgegriffen. Übertragen auf die BWI 2012 Gesamtwaldfläche entspricht die absolute Stillegungsfläche von Engel (2013) 2,1\%. Zur NMF-Umsetzung wird daher eine Erhöhung der Waldflächenkategorie „Wald ohne forstliche Bewirtschaftung" um 2,9\%-Punkte auf insgesamt $5 \%$, gemäß der Zielsetzung der Biodiversitätsstrategie (BMU, 2007) unterstellt. Da in Hinsicht auf die Waldstruktur der stillgelegten Flächen im "Status quo" und in der "Neuen Multifunktionalität" (z. B. Altersklassenstruktur, Flächenanteil der Holzartengruppen) die Ergebnisse des NWE5-Projekts (Wildmann, 2013) nicht präzise übertragbar sind, wurde für die Berechnungen eine gleichmäßige Verteilung der Flächen mit natürlicher Waldentwicklung über alle Altersklassen und Holzartengruppen der BWI 2012 angenommen.

Ein weiteres Ziel der Biodiversitätsstrategie ist die Entstehung von Wildnisgebieten auf $2 \%$ der Fläche Deutschlands (BMU, 2007). Es wird jedoch nicht spezifiziert, wie hoch der Flächenanteil von Wildnisgebieten im Wald sein soll und ob die Wildnisgebiete zusätzlich zu den $5 \%$ stillgelegten Waldflächen ausgewiesen oder Teil davon werden sollen. Daher wird im Rahmen dieser Studie unterstellt, dass sich die Wildnis im Wald innerhalb der "Waldfläche mit natürlicher Waldentwicklung" (5\%-Ziel) findet.

\subsubsection{Wald mit Naturschutzvorrangfunktion}

Für den Flächenumfang der "Waldflächen mit Naturschutzvorrangfunktion" und für die Operationalisierung der naturschutzfachlichen Maßnahmen auf diesen Flächen müssen weitere Annahmen getroffen werden. Zur Bestimmung des Anteils der NMF-Waldflächenkategorie "Waldflächen mit Naturschutzvorrangfunktion" wird auf die Studie von Polley (2009) zurückgegriffen. Für die intensiven Schutzgebietskategorien (Naturschutzgebiete, Nationalparke, Biosphärenreservate, Natura 2000-Gebiete) weist Polley (2009) einen Anteil von $25 \%$ aus, die sich jedoch untereinander teilweise überlagern können. Die Natura 2000-Schutzgebiete hatten im Jahr 2009 bspw. einen Anteil von $24 \%$ und die Naturschutzgebiete einen Anteil von $6 \%$ an der deutschen Waldfläche (Polley, 2009). Innerhalb der Natura 2000-Gebiete befinden sich 816.620 ha FFH-Waldlebensraumtypen (entspricht 7,7 \% der bestockten Waldfläche abzüglich der „Waldflächen ohne forstliche Bewirtschaftung") für die größtenteils naturschutzfachliche Maßnahmenplanungen vorliegen (BfN, 2012). Die neueren Entwicklungen hinsichtlich der Fläche ohne forstliche Nutzungen im Wald, und die Flächenanteile sowie der Grad der Überlagerung einzelner Schutzkategorien sind unbekannt. Es ist anzunehmen, dass seit dem Jahr 2009 noch weitere Schutzgebietsflächen mit intensivem Schutzstatus hinzugekommen sind.

Für unsere Berechnungen wird daher in Anlehnung an die von Polley (2009) erarbeiteten Ergebnisse unterstellt, dass zur Umsetzung des NMF-Konzeptes $25 \%$ der deutschen Gesamtwaldfläche als „Waldflächen mit Naturschutzvorrangfunktion" ausgewiesen werden. Es wird jedoch angenommen, dass naturschutzfachliche Maßnahmenplanungen im "Status quo" aktuell erst auf einem Teil der "Waldflächen mit Naturschutzvorrangfunktion" umgesetzt werden. Diese Teilflächen beinhalten die FFH-Waldlebensraumtypen (7,7 \%) einschließlich ihrer Baumartenverteilung. Es sind aber in den deutschen Wäldern weitere Schutzflächen vorhanden, auf denen naturschutzfachliche Maßnahmen umgesetzt werden könnten, so möglicherweise auf Flächen der Deutschen Bundesstiftung Umwelt oder des Naturschutzbunds Deutschland. Um diese Flächen abzudecken, wurden die 816.620 ha Schutzfläche für FFH-Waldlebensraumtypen pauschal, über alle Baumarten, auf 900.000 ha ausgeweitet. Für die Waldbehandlungsvariante "Status quo" errechnet sich folglich, dass auf rund $8,5 \%$ des bestockten, begehbaren Holzbodens naturschutzfachliche Maßnahmenplanungen auf der NMFWaldflächenkategorie „Wald mit Naturschutzvorrangfunktion" realisiert sind. Diese Maßnahmenfläche wird in der Waldbehandlungsvariante "Neue Multifunktionalität" von 8,5\% auf $25 \%$ erweitert. Für die jeweils restliche Fläche wird angenommen, dass diese im "Status quo" regulär forstlich bewirtschaftet wird. Vor diesem Hintergrund werden in der Waldbehandlungsvariante "Status quo" $8,5 \%$ und "Neue Multifunktionalität" $25 \%$ für die Flächenmodellierung mit speziellem Schutzzweck unterstellt.

Während Mindererträge in Wäldern ohne forstliche Nutzung (Stilllegung) durch eine Bewertung des Flächentotalausfalls für die forstliche Nutzung leicht abschätzbar sind, müssen für die NMF-Waldflächenkategorie „Waldflächen mit Naturschutzvorrangfunktion" naturschutzfachliche Maßnahmen abgeleitet werden, die auf eine Verbesserung des Waldnaturschutzniveaus abzielen und gleichzeitig Einschränkungen 
für die forstliche Nutzung darstellen. Auf den „Waldflächen mit Naturschutzvorrangfunktion" könnten unterschiedlichste Arten- und Biotopschutzmaßnahmen sowie auch kulturhistorische Nutzungsformen (z. B. Nieder- und Mittelwaldbewirtschaftung) umgesetzt werden. Aus forschungsökonomischen Gründen konnte diese Vielzahl unterschiedlicher Schutzmaßnahmen nicht einzeln bewertet werden. Aus diesem Grund unterstellen wir für unsere Berechnungen, dass die spezifischen Schutzzwecke durch Umsetzung von typischen FFH-Maßnahmen für Waldlebensraumtypen abgedeckt sind.

Auf Basis von Rosenkranz et al. (2012), Seintsch et al. (2012), Wagner und Jönsson (2001), FVA (2007) und Schmack et al. (2013) wurden vier naturschutzfachliche FFH-Maßnahmen ausgewählt, aus denen im Besonderen Minderertragstatbestände bzw. Nutzungsverzichte für die forstliche Rohholzerzeugung resultieren:

1. Erhalt / Erhöhung der lebensraumtypischen Artenzusammensetzung

2. Erhalt / Erhöhung des Anteils vorhandener Habitatbäume

3. Erhalt / Erhöhung des Totholzanteils

4. Erhalt / Erhöhung des Altholzanteils

Diese vier FFH-Maßnahmen dürften die Naturschutzanforderungen für eine Umsetzung höherer naturschutzfachlicher Standards in der NMF-Flächenkategorie „Waldflächen mit Naturschutzvorrangfunktion" hinreichend abdecken (Seintsch et al., 2012). Die aufgeführten naturschutzfachlichen Maßnahmen können Auswirkungen für Waldbesitzer haben, die zu Mehraufwand oder Mindererträgen führen (Seintsch et al., 2012; Rosenkranz et al., 2014). Diese Auswirkungen sowie die Operationalisierung der Maßnahmen zur Berechnung mit dem Strugholtz-Englert-Modell sind für beide Waldbehandlungsvarianten im Folgenden beschrieben:

Einschränkung der Baumartenwahl: Eine Erhöhung des Laubholzanteils kann in zweifacher Hinsicht zu Ertragseinbußen führen. Zum einen wird auf das ertragsstärkere Nadelholz verzichtet. Des Weiteren resultieren aus einem naturschutzorientierten Waldumbau zusätzliche Investitionskosten (z. B. Kultur- und Läuterungskosten). Auf Grundlage der FFH-Bewertungsschemata für Waldlebensraumtypen der Länder wurden $80 \%$ Laubholzanteil als Ziel auf den „Waldflächen mit Naturschutzvorrangfunktion" unterstellt (entspricht der FFHZielvorgabe für den Erhaltungszustand B) (Rosenkranz et al., 2012). Der Laubholzanteil in der Waldbehandlungsvariante "Status quo" beträgt derzeit rund $90 \%$ auf den „Waldflächen mit Naturschutzvorrangfunktion" und muss folglich nicht weiter angehoben werden. Bei einer Umsetzungsvariante "Neue Multifunktionalität" beträgt der Laubholzanteil auf den $25 \%$ „Waldflächen mit Naturschutzvorrangfunktion" $63 \%$. In der Waldbehandlungsvariante "Neue Multifunktionalität" muss der Laubholzanteil folglich um17 \%-Punkte angehoben werden.

Punktueller und kleinflächiger Nutzungsverzicht: Die dauerhafte Ausweisung von Habitatbäumen oder Habitatbaumgruppen im Wald kommt einer Stillegung und damit einer Verringerung der forstlichen Produktionsfläche und eines Verlusts an Rohholzaufkommen, gleich. Die BWI 2012 weist insgesamt 23,7 Millionen (Mio.) Habitatbäume aus. Davon sind jedoch 22 Mio. Höhlenbäume, die durch Spechtaktivitäten oder abgebrochene Äste entstehen. Die restlichen 1,74 Mio. Bäume bestehen aus Horstbäumen, die leicht identifizierbar sein dürften, sowie aus dauerhaft markierten Habitatbäumen. Wir nehmen an, dass es sich hierbei überwiegend um Altbäume ab dem Alter 100 handelt. Altbäume ab dem Alter 100 nehmen gemäß der BWI 2012 derzeit eine Fläche von rund 2,28 Mio. ha ein. Auf die Gesamtfläche der nicht aus der Nutzung genommenen Holzbodenfläche verteilt entsprechen die 1,74 Mio. Habitat- und Höhlenbäume rund 0,76 Habitatbäumen pro Hektar auf Waldflächen ab dem Alter 100. Wir unterstellen hierbei, dass auf "Waldflächen mit Naturschutzvorrangfunktion" mehr Habitatbäume stehen als im Wirtschaftswald. Für die Waldbehandlungsvariante "Status quo" schätzen wir daher 2 Habitatbäume pro Hektar auf „Waldflächen mit Naturschutzvorrangfunktion" sowie 0,5 Habitatbäume pro Hektar im Wirtschaftswald. Auf Grundlage der Maßnahmenplanungen und der Erhaltungszustandsbewertungen in FFH-Gebieten (Rosenkranz et al., 2012) wurde in der Waldbehandlungsvariante "Neue Multifunktionalität" eine durchschnittliche Erhöhung von 2 auf 5 Habitatbäume pro Hektar in der NMF-Flächenkategorie „Wald mit Naturschutzvorrangfunktion" unterstellt. Als vereinfachende Annahme wurde unterstellt, dass die dauerhaft ausgewiesenen Habitatbäume über den 200-jährigen Simulationszeitraum vital bleiben und nicht zur Nachlieferung des Totholzvorrates beitragen.

Belassen von Totholz: In den FFH-Maßnahmenplanungen wird der Erhalt eines definierten Totholzvorrates zusätzlich zur Ausweisung von Habitatbäumen gefordert (Rosenkranz et al., 2012). Aufgrund der natürlichen Holzzersetzung bedingt der Erhalt dieses Totholzvorrates kontinuierliche Nachliefermengen, welche einen Nutzungsverzicht für Waldbesitzer darstellen. Zur Abschätzung dieses Nutzungsverzichtes wurde in unseren Berechnungen die Annahme getroffen, dass natürliche Mortalität im Kontext der Totholznachliefermengen zu vernachlässigen ist. Die BWI 2012 weist eine durchschnittliche Menge stehenden und liegenden Totholzes von $20,6 \mathrm{~m}^{3}$ pro Hektar Wald aus. Abfuhrreste und Wurzelanläufe wurden von dieser Gesamttotholzmenge abgezogen. Diese Mengen dürften naturschutzfachlich einen geringen Wert haben und sind auch nicht als Nutzungsverzicht der Rohholzproduktion zu bewerten. Somit wurde für unsere Studie ein Durchschnittswert von 14,7 $\mathrm{m}^{3}$ Totholz pro Hektar unterstellt, der in der Waldbehandlungsvariante "Status quo" gehalten wird. In Anlehnung an die Arbeiten von Winter (2010) wurde bei NMF-Umsetzung zur Anhebung des Waldnaturschutzniveaus für die „Waldflächen mit Naturschutzvorrangfunktion" ein Zielwert von $50 \mathrm{~m}^{3}$ Totholz pro Hektar angenommen. Für die Simulation 
wurde hierbei unterstellt, dass die fehlende Totholzmenge von $34,3 \mathrm{~m}^{3}$ in den ersten 10 Jahren nach Simulationsbeginn aufgebaut und der Zieltotholzvorrat von $50 \mathrm{~m}^{3}$ dann in den Folgejahren konstant gehalten wird.

Erhöhung der Produktionszeit: In beiden Waldbehandlungsvarianten wurde auf den „Waldflächen mit Naturschutzvorrangfunktion" zur Erhöhung des Altholzanteils pauschal eine Erhöhung der Produktionszeiten um 20 Jahre unterstellt. Die Produktionszeit der Holzartengruppe Buche wurde damit von 150 Jahre auf 170 Jahre, die der Eiche von 180 Jahre auf 200 Jahre, die der Fichte von 120 Jahre auf 140 Jahre und die der Kiefer von 140 Jahre auf 160 Jahre verlängert. Die Erhöhung der Produktionszeit führt zu einem Aufschub der Endnutzung und dadurch zu zeitverzögerten Erträgen. Die aus der forstlichen Praxis bekannte altersbedingte Holzqualitätsentwertung der Bäume, beispielsweise durch Rotkern oder Fäule, und die damit verbundene Veränderung der Sortimentsstruktur konnte aufgrund des geringen Kenntnisstandes zu den Zusammenhängen nicht bewertet werden. Da auf Basis des TBN-Forst keine Informationen zu den sortiments- und dimensionsspezifischen Holzerlösen vorliegen, konnte ebenfalls nicht berücksichtigt werden, dass bei einigen Nadelholzarten für starkes, sägefähiges Stammholz Preisabschläge in der Praxis bestehen.

Erhöhung der Holzerntekosten: Durch Arbeitssicherheitsmaßnahmen (z. B. seilunterstütze Fällung) wegen totholzreicher Kronen von Altholz- und Habitatbäumen sowie durch die Erhöhung von Rückegassenabständen zur Schonung des Waldbodens können sich Holzerntekosten erhöhen. In Anlehnung an Seintsch et al. (2012) wurden in beiden Waldbehandlungsvarianten Mehrkosten von $1 € /$ Efm pro Habitatbaum auf der Fläche für Arbeitssicherheitsmaßnahmen bei der Ernte der umstehenden Bäume unterstellt. Für den „Waldflächenanteil mit Naturschutzvorrangfunktion" wurde weiterhin unterstellt, dass die Abstände der Rückegassen in beiden Waldbehandlungsvarianten (somit auch auf den zusätzlichen Naturschutzvorrangflächen in der Waldbehandlungsvariante "Neue Multifunktionalität") aus naturschutzfachlicher Sicht bereits ausreichend und daher nicht erhöht werden müssen. Es entstehen auf diesen Flächen daher keine zusätzlichen Rückungskosten.

Erhöhung der Verwaltungskosten: Bei einer Anhebung des Waldnaturschutzniveaus können zusätzliche Verwaltungskosten in der Holzernte z. B. durch vermehrten Aufwand bei der Hiebsplanung oder durch Abstimmungen mit den Naturschutzverwaltungen entstehen. Der Kenntnisstand zu den Veränderungen der Verwaltungskosten von Forstbetrieben bei einer Anhebung des Waldnaturschutzniveaus oder nach Ausweisung von Schutzgebietskulissen ist bisher gering. Von Seintsch et al. (2012: 86) wurden zusätzliche jährliche Verwaltungskosten für die Waldbewirtschaftung in FFH-Buchenlebensraumtypen in 18 von 21 untersuchten Fallbeispielsbetrieben mit im Mittel 9,10 €/ha erhoben. Bei einer ebenfalls von Seintsch et al. (2012: 86) durchgeführten bundesweiten Online-Befragung wurde ein zusätzlicher Verwaltungsaufwand für den jährlichen Betriebsvollzug auf FFH-Gebietsflächen von $28 \%$ der befragten Forstbetriebe ( 25 Betriebe) mit durchschnittlich $12,16 € /$ ha pro Jahr ermittelt (Befragung erfolgte im laufenden FFH-Umsetzungsprozess). Für die eigene Untersuchung werden erhöhte Verwaltungskosten von 9,1€/ha/Jahr innerhalb der Waldflächenkategorie Wald mit Naturschutzvorrangfunktion unterstellt.

\subsubsection{Wirtschaftswald mit naturschutzfachlichen Mindeststandards}

Wie in den Kapitel 2.2.1 beschrieben, wird für die Studie im "Status quo" ein "Waldflächenanteil ohne forstliche Bewirtschaftung" von 2,1\% angenommen. Weiterhin wird angenommen, dass im "Status quo" die "Waldfläche mit Naturschutzvorrangfunktion" mit umgesetzten Naturschutzmaßnahmen 8,5 \% der Gesamtwaldfläche beträgt. Diese Maßnahmenfläche wird in der Waldbehandlungsvariante "Neue Multifunktionalität" auf $25 \%$ ausgeweitet. (Kapitel 2.2.2). Der Flächenanteil des "Wirtschaftswalds mit naturschutzfachlichen Mindeststandards" beträgt somit in der Waldbehandlungsvariante "Status quo" 89,4\% der Gesamtwaldfläche. Für die Berechnung der Waldbehandlung im "Status quo" werden keine Änderungen der forstlichen Bewirtschaftung unterstellt. Durch die Erweiterung der Flächenkategorien "Waldflächen mit Naturschutzvorrangfunktion" auf $25 \%$ und "Waldfläche ohne forstliche Bewirtschaftung" auf $5 \%$, reduziert sich die Fläche der Kategorie "Wirtschaftswald mit naturschutzfachlichen Mindeststandards" in der Bewirtschaftungsvariante "Neue Multifunktionalität" auf $70 \%$ des bestockten Holzbodens. Auf diesen werden zusätzliche naturschutzfachliche Maßnahmenplanungen zur Implementierung des NMF-Konzeptes unterstellt.

Für die Operationalisierung der naturschutzfachlichen Maßnahmenplanungen auf den Flächen des "Wirtschaftswalds mit naturschutzfachlichen Mindeststandards" wurden im Wesentlichen Annahmen auf Basis der Waldstrategie 2020 (BMELV, 2011) und der Biodiversitätsstrategie (BMU, 2007) getroffen. Die Biodiversitätsstrategie nennt bspw. als Ziele für die Waldbewirtschaftung bis zum Jahr 2020 das Vorhandensein eines in Menge und Qualität ausreichenden Alt- und Totholzanteils, die Reduktion nicht-standortheimischer Baumarten sowie eine Bewirtschaftung von 80 \% der Waldfläche nach forstlichen Zertifizierungssystemen. Aus diesen Zielen wurden nachfolgende vier naturschutzfachliche Maßnahmenbereiche abgeleitet:

1. Anbau von standortgerechten Baumarten

2. Punktuelle und kleinflächige Stilllegung von Waldflächen (z. B. Habitatbäumen)

3. Erhöhung des Totholzvorrates

4. Erhöhung des Altholzanteils durch Erhöhung der Produktionszeit

Diese naturschutzfachlichen Maßnahmen wurden auf Basis der nachfolgend aufgeführten Literatur operationalisiert. War keine Literatur vorhanden, so wurden eigene Annahmen getroffen: 
Einschränkung der Baumartenwahl: In der Waldstrategie 2020 wird der Anbau standortgerechter und überwiegend heimischer Baumarten gefordert, in der Biodiversitätsstrategie die Vermehrung standortheimischer Arten sowie die kontinuierliche Reduktion nicht standortheimischer Arten (BMU, 2007; BMELV, 2011). Im Rahmen der Studie wird aus diesen Zielen abgeleitet, dass der Anteil von Laubholz erhöht werden soll. In Anlehnung an Regelungen in Waldbaukonzepten ausgewählter Landesforstverwaltungen (Landesforst Mecklenburg-Vorpommern, 1996; HessenForst, 2008 und Niedersächsische Landesforsten, 2011) wird ein durchschnittlicher Anteil von $60 \%$ Laubholz auf "Wirtschaftswaldflächen mit naturschutzfachlichen Mindeststandards" bei einer NMF-Implementierung unterstellt. Außer diesen waldbaulichen Zielwerten in den Waldbaukonzepten der Länder lagen den Autoren keine weiteren operationalen Zielformulierungen zur Erhöhung des Laubholzanteils vor. Der Laubholzanteil im „Status quo" beträgt $44 \%$. In der Waldbehandlungsvariante „Neue Multifunktionalität" wird der Laubholzanteil daher um $16 \%$-Punkte angehoben. Bei dieser Annahme handelt es sich um einen Durchschnittswert für den gesamten deutschen Wald, welcher nicht zwangsläufig von jeder Waldeigentumsart durchschnittlich zu erfüllen wäre.

Punktueller und kleinflächiger Nutzungsverzicht: Auf Grundlage der Alt- und Totholzprogramme von zehn Landesforstverwaltungen (Landesforst MecklenburgVorpommern, 2002; SaarForst, 2003; Forst Brandenburg, 2004; BaySF, 2009; Schmalfuß et al., 2010; HessenForst, 2011; Landesforsten Rheinland-Pfalz, 2011; Niedersächsische Landesforsten, 2011; Landesforstbetrieb Sachsen Anhalt, 2014; Thüringen-Forst, ohne Jahr) wurden bei Umsetzung des NMF-Konzeptes 3 Habitatbäume pro Hektar in Beständen ab dem Alter 100 Jahre unterstellt. Ausgehend von im "Status quo" unterstellten 0,5 Habitatbäumen pro Hektar resultiert hieraus eine Erhöhung um 2,5 Habitatbäume pro Hektar in der Waldbehandlungsvariante "Neue Multifunktionalität".

Steigerung des Totholzanteils: In Anlehnung an die Studie von Winter (2010) wird in der Waldbehandlungsvariante "Neue Multifunktionalität", eine Erhöhung des Totholzvorrates von $14,7 \mathrm{~m}^{3}$ pro Hektar im "Status quo" auf $30 \mathrm{~m}^{3}$ pro Hektar unterstellt. Dabei wurde zunächst der Aufbau des Zielvorrats in den ersten 10 Jahren nach Simulationsbeginn berechnet und der Zieltotholzvorrat von $30 \mathrm{~m}^{3}$ dann in den Folgejahren konstant gehalten.

Erhöhung des Produktionszeit: Für unsere Berechnungen unterstellen wir, dass in beiden Waldbehandlungsvarianten keine Erhöhung der Umtriebszeit auf "Wirtschaftswaldflächen mit naturschutzfachlichen Mindeststandards" stattfindet, sondern dass der Altholzbedarf durch die Erhöhung der Habitatbaumanzahlen abgedeckt wird.

Erhöhung der Holzerntekosten: Wie bereits bei der Operationalisierung der naturschutzfachlichen Maßnahmen auf den „Waldflächen mit Naturschutzvorrangfunktion" wird in beiden Waldbehandlungsvarianten eine
Erhöhung der Holzerntekosten um $1 € /$ Efm pro Habitatbaum für Arbeitssicherheitsmaßnahmen unterstellt. Der Abstand von dauerhaften Rückegassen im Wirtschaftswald wird im "Status quo" auf 20 bis $30 \mathrm{~m}$ geschätzt. In der Waldbewirtschaftungsvariante "Neue Multifunktionalität" werden, in Anlehnung an aktuelle Zertifizierungsrichtlinien eine Erhöhung der Rückegassenabstände auf $40 \mathrm{~m}$ unterstellt. In Folge wurden pauschal zusätzliche Holzerntekosten von $3 € / E f m$ angenommen (AFL Niedersachsen, 2010; FSC, 2012).

Erhöhung der Verwaltungskosten: In der Waldbehandlungsvariante "Neue Multifunktionalität" wird auf den „Wirtschaftswaldflächen mit naturschutzfachlichen Mindeststandards" ebenfalls eine Erhöhung der Verwaltungskosten um 9,1 €/ha in Folge des erhöhten Waldnaturschutzniveaus für bspw. aufwendigere Hiebsplanung oder Abstimmung von forstlichen Maßnahmen mit Naturschutzverwaltungen unterstellt.

Die vorgestellten Annahmen und zentralen Datenquellen zur Operationalisierung des NMF-Konzeptes sind für die beiden Waldbehandlungsvarianten in Tabelle 1 nochmals vergleichend gegenübergestellt.

\section{Ergebnisse}

\subsection{Rohholzaufkommen}

Bei einer Weiterführung der Waldbewirtschaftung nach dem "Status quo" würden in den nächsten 200 Jahren auf den „Waldflächen mit Naturschutzvorrangfunktion" zwischen 5,5 und 7,0 Efm/ha/Jahr, im Mittel 6,3 Efm/ha/Jahr, an Rohholz eingeschlagen werden. Im „Wirtschaftswald mit naturschutzfachlichen Mindeststandards" sind es zwischen 7,2 und 8,4 $\mathrm{Efm} / \mathrm{ha} / \mathrm{Jahr}$, im Mittel 7,8 Efm/ha/Jahr. Für die gesamte deutsche Holzbodenfläche berechnet sich ein Rohholzaufkommen zwischen 6,9 und 8,1 Efm/ha/Jahr, im Mittel 7,5 Efm/ ha/Jahr (Tabelle 2). Bei einer Fortführung des "Status quo" entspricht dies insgesamt einem Rohholzaufkommen zwischen 73,4 und 85,7 Mio. Efm/Jahr und im Mittel 79,6 Mio. Efm/Jahr.

Bei einer Umsetzung des NMF-Konzeptes zur Anhebung des Waldnaturschutzniveaus reduziert sich das Rohholzaufkommen auf der gesamten deutschen Holzbodenfläche auf 5,7 bis 7,2 Efm/ha/Jahr, im Mittel um 6,4 Efm/ha/Jahr. In der Waldbewirtschaftungsvariante „Neue Multifunktionalität" können auf den Flächenkategorien „Wirtschaftswald mit naturschutzfachlichen Mindeststandards" dann im Mittel nur noch 7,0 Efm/ha/Jahr (zwischen 6,5 und 7,8 Efm/ha/Jahr) und auf den "Waldflächen mit Naturschutzvorrangfunktion“ im Mittel nur noch 6,0 Efm/ha/Jahr (zwischen 4,7 bis 7,0 Efm/ ha/Jahr) Rohholz eingeschlagen werden (Tabelle 2). Auf der Gesamtwaldfläche entspricht dies bei einer Umsetzung der "Neue Multifunktionalität" einem Rohholzaufkommen zwischen 60,6 und 76,5 Mio. Efm/Jahr und im Mittel 68,4 Mio. Efm/Jahr. In der Differenz der beiden Waldbehandlungsvarianten ergibt sich ein Verlust an Rohholzaufkommen von 7,6 bis 14,9 Mio. Efm/Jahr, im Mittel von rund 11,2 Mio. Efm/ Jahr durch eine Erhöhung des Waldnaturschutzniveaus nach dem NMF-Konzept (Tabelle 2). 


\section{Tabelle 1}

Operationalisierung der Waldbewirtschaftungsvarianten "Status quo" und "Neue Multifunktionalität" für die Modellrechnung

\begin{tabular}{|c|c|c|c|c|}
\hline & \multicolumn{2}{|c|}{ "Status quo" } & \multicolumn{2}{|c|}{ „Neue Multifunktionalität" } \\
\hline & Maßnahmen & Quelle & Maßnahmen & Quelle \\
\hline $\begin{array}{l}\text { Wald ohne forstliche } \\
\text { Bewirtschaftung }\end{array}$ & $2,1 \%$ & Engel (2013) & $5 \%$ & BMU (2007) \\
\hline $\begin{array}{l}\text { Wald mit Naturschutz- } \\
\text { vorrangfunktion }\end{array}$ & $8,5 \%$ & $\begin{array}{l}\text { Polley (2009), } \\
\text { BfN (2012) }\end{array}$ & $25 \%$ & Polley (2009) \\
\hline Einschränkung der Baumartenwahl & $\begin{array}{l}\text { Keine Erhöhung des } \\
\text { Laubholzanteils (IST: } 90 \% \text { ) }\end{array}$ & BMEL (2012) & $\begin{array}{l}\text { Erhöhung des Laubholz- } \\
\text { anteils von } 63 \% \text { auf } 80 \%\end{array}$ & $\begin{array}{l}\text { BMEL (2012), } \\
\text { Rosenkranz et al. (2012) }\end{array}$ \\
\hline Punktueller Nutzungsverzicht & $\begin{array}{l}\text { Keine Erhöhung der } \\
\text { Habitatbaumanzahl } \\
\text { (IST: } 2 \text { Stück/ha) }\end{array}$ & $\begin{array}{l}\text { Annahme auf Basis von } \\
\text { BMEL (2012) }\end{array}$ & $\begin{array}{l}\text { Erhöhung der Habitat- } \\
\text { baumanzahl von } \\
2 \text { auf } 5 \text { Stück/ha }\end{array}$ & Rosenkranz et al. (2012) \\
\hline Belassen von Totholz & $\begin{array}{l}\text { Keine Erhöhung des Tot- } \\
\text { holzanteils (IST: } 14,7 \mathrm{~m}^{3} / \mathrm{ha} \text { ) }\end{array}$ & BMEL (2012) & $\begin{array}{l}\text { Erhöhung des Totholzanteils } \\
\text { von } 14,7 \mathrm{~m}^{3} \text { auf } 50 \mathrm{~m}^{3}\end{array}$ & $\begin{array}{l}\text { Annahme auf Basis von } \\
\text { Winter (2010) }\end{array}$ \\
\hline Erhöhung der Produktionszeit & $\begin{array}{l}\text { Erhöhung um } 20 \text { Jahre für } \\
\text { alle Baumarten }\end{array}$ & Seintsch et al. (2012) & $\begin{array}{l}\text { Erhöhung um } 20 \text { Jahre für } \\
\text { alle Baumarten }\end{array}$ & Seintsch et al. (2012) \\
\hline Erhöhung der Holzerntekosten & $\begin{array}{l}2 € / E f m \\
\text { (für } 2 \text { Habitatbäume /ha) }\end{array}$ & Seintsch et al. (2012) & $\begin{array}{l}5 € / \text { Efm } \\
\text { (für } 5 \text { Habitatbäume /ha) }\end{array}$ & Seintsch et al. (2012) \\
\hline Erhöhung der Verwaltungskosten & $\begin{array}{l}\text { Erhöhte Verwaltungskosten } \\
\text { von } 9,1 € / \mathrm{ha} / J a h r\end{array}$ & Seintsch et al. (2012) & $\begin{array}{l}\text { Erhöhung der Verwaltungs- } \\
\text { kosten um } 9,1 € / \text { ha/Jahr }\end{array}$ & Seintsch et al. (2012) \\
\hline $\begin{array}{l}\text { Wirtschaftswald mit naturschutz- } \\
\text { fachlichen Mindeststandards }\end{array}$ & $89,4 \%$ & Eigene Berechnung & $70 \%$ & Eigene Berechnung \\
\hline Einschränkung der Baumartenwahl & $\begin{array}{l}\text { Keine Erhöhung des } \\
\text { Laubholzanteils (IST: } 44 \% \text { ) }\end{array}$ & BMEL (2012) & $\begin{array}{l}\text { Erhöhung des Laubholz- } \\
\text { anteils von } 44 \% \text { auf } 60 \%\end{array}$ & $\begin{array}{l}\text { BMU (2007), } \\
\text { BMELV (2011), } \\
\text { BMEL (2012), } \\
\text { Rosenkranz et al. (2012), } \\
\text { Waldbaurichtlinien aus } \\
3 \text { Bundesländern } \\
\text { (siehe Text) }\end{array}$ \\
\hline Punktueller Nutzungsverzicht & $\begin{array}{l}\text { Keine Erhöhung der } \\
\text { Habitatbaumanzahl } \\
\text { (IST: 0,5 Stk./ha) }\end{array}$ & $\begin{array}{l}\text { Annahme auf Basis von } \\
\text { BMEL (2012) }\end{array}$ & $\begin{array}{l}\text { Erhöhung der Habitat- } \\
\text { baumanzahl von 0,5 auf } 3 \\
\text { Stück/ha }\end{array}$ & $\begin{array}{l}\text { Annahme auf Basis von } \\
\text { Waldbaurichtlinien sowie } \\
\text { Alt- und Totholzkon- } \\
\text { zepten aus } 10 \text { Bundes- } \\
\text { ländern (siehe Text) }\end{array}$ \\
\hline Belassen von Totholz & $\begin{array}{l}\text { Keine Erhöhung des Tot- } \\
\text { holzanteils (IST: } 14,7 \mathrm{~m}^{3} / \mathrm{ha} \text { ) }\end{array}$ & BMEL (2012) & $\begin{array}{l}\text { Erhöhung des Totholzanteils } \\
\text { von } 14,7 \mathrm{~m}^{3} \text { auf } 30 \mathrm{~m}^{3}\end{array}$ & $\begin{array}{l}\text { Annahme auf Basis von } \\
\text { Winter (2010) }\end{array}$ \\
\hline Erhöhung der Produktionszeit & $\begin{array}{l}\text { Keine Erhöhung der } \\
\text { Produktionszeit }\end{array}$ & Seintsch et al. (2012) & $\begin{array}{l}\text { Keine Erhöhung der } \\
\text { Produktionszeit }\end{array}$ & Seintsch et al. (2012) \\
\hline Erhöhung der Holzerntekosten & $\begin{array}{l}0,5 € / \text { Efm } \\
\text { (für } 0,5 \text { Habitatbäume /ha) }\end{array}$ & Seintsch et al. (2012) & $\begin{array}{l}6 € / \text { Efm } \\
\text { (für } 3 \text { Habitatbäume /ha } \\
\text { sowie für Erhöhung der } \\
\text { Rückegassenabstände } \\
\text { auf } 40 \mathrm{~m} \text { ) }\end{array}$ & $\begin{array}{l}\text { AFL Niedersachsen (2010), } \\
\text { FSC (2012), } \\
\text { Seintsch et al. (2012) }\end{array}$ \\
\hline Erhöhung der Verwaltungskosten & $\begin{array}{l}\text { Keine Erhöhung der } \\
\text { Verwaltungskosten }\end{array}$ & Seintsch et al. (2012) & $\begin{array}{l}\text { Erhöhung der Verwaltungs- } \\
\text { kosten um } 9,1 € / \text { ha/Jahr }\end{array}$ & Seintsch et al. (2012) \\
\hline
\end{tabular}

Die Differenzen des jährlichen Rohholzaufkommens zwischen den Waldbehandlungsvarianten "Status quo" und "Neue Multifunktionalität" sind untergliedert nach Holzartengruppen für den 200-jährigen Betrachtungszeitraum in Abbildung 1 dargestellt. Auf den ersten Blick wird der Rohholzaufkommensverlust in allen Holzartengruppen in der ersten Berechnungsperiode deutlich. Dieser Verlust erhöht sich für das Nadelholz (Holzartengruppen Fichte und Kiefer in Summe) bis zur achten Periode des Betrachtungszeitraums und sinkt danach wieder leicht ab. Für die
Holzartengruppen Buche und Eiche erhöht sich ab der dritten Periode das Rohholzaufkommen und steigt weiter an.

Schwankungen in der Höhe des Rohholzaufkommens ergeben sich grundsätzlich in beiden Waldbehandlungsvarianten durch die Verteilung der Baumarten und die Altersklassenstruktur sowie die unterstellten Produktionszeiten. Die Differenzen des Rohholzaufkommens zwischen den beiden Waldbehandlungsvarianten resultieren aus unterschiedlichen Flächenanteilen von "Wald ohne forstliche Bewirtschaftung" und dauerhaft ausgewiesenen Habitatbäumen 
Tabelle 2

Vergleich des minimalen, maximalen und mittleren Rohholzaufkommens in Efm pro Hektar und Jahr in der 200-jährigen Simulationsperiode für die Waldbehandlungsvarianten "Status quo" und "Neue Multifunktionalität" sowie die Gesamtdifferenz aus beiden Waldbehandlungsvarianten in Mio. Efm je Jahr

\begin{tabular}{|c|c|c|c|}
\hline Gesamteinschlag [Efm/ha/Jahr] & Mittelwert & Minimum & Maximum \\
\hline \multicolumn{4}{|l|}{ „Status quo" } \\
\hline Wald ohne forstliche Bewirtschaftung (2,1\%) & 0,0 & 0,0 & 0,0 \\
\hline Wald mit Naturschutzvorrangfunktion (8,5\%) & 6,3 & 5,5 & 7,0 \\
\hline Wirtschaftswald mit naturschutzfachlichen Mindeststandards $(89,4 \%)$ & 7,8 & 7,2 & 8,4 \\
\hline Status quo Gesamt & 7,5 & 6,9 & 8,1 \\
\hline \multicolumn{4}{|l|}{ „Neue Multifunktionalität“ } \\
\hline Wald ohne forstliche Bewirtschaftung (5\%) & 0,0 & 0,0 & 0,0 \\
\hline Wald mit Naturschutzvorrangfunktion ( $25 \%$ ) & 6,0 & 4,7 & 7,0 \\
\hline Wirtschaftswald mit naturschutzfachlichen Mindeststandards (70 \%) & 7,0 & 6,5 & 7,8 \\
\hline Neue Multifunktionalität Gesamt & 6,4 & 5,7 & 7,2 \\
\hline Differenz Status quo/ Neue Multifunktionalität [Mio. Efm/Jahr] & $-11,2$ & $-7,6$ & $-14,9$ \\
\hline
\end{tabular}

sowie der Höhe des Nutzungsverzichtes für die Totholznachliefermengen als unmittelbar wirkende Naturschutzmaßnahmen. Weiterhin wirken über den gesamten Simulationszeitraum die abweichenden Umtriebszeiten. Mittel- bis langfristig wirkt hingegen der laubholzorientierte Waldumbau auf die Differenzen des Rohholzaufkommens.

Das Rohholzaufkommen der Holzartengruppen Buche und Eiche erhöht sich ab der dritten Periode aufgrund der Umbaumaßnahmen hin zu einem höheren Laubholzanteil in der Waldbehandlungsvariante "Neue Multifunktionalität" auf den "Waldflächen mit Naturschutzvorrangfunktion“ sowie dem "Wirtschaftswald mit naturschutzfachlichen Mindeststandards". Die zusätzlichen Holzmengen in diesen
Holzartengruppen kompensieren jedoch nicht den Verlust an Rohholzaufkommen in den Holzartengruppen Fichte und Kiefer (vgl. auch Ermisch et al., 2013).

\subsection{Deckungsbeiträge}

Tabelle 3 zeigt den mittleren, minimalen und maximalen Deckungsbeitrag pro Hektar und Jahr innerhalb der 200-Jährigen Simulationsperiode für die Waldbehandlungsvarianten "Status quo" und "Neue Multifunktionalität" sowie die Gesamtdifferenz aus beiden Waldbehandlungsvarianten in Milliarden (Mrd.) Euro je Jahr.

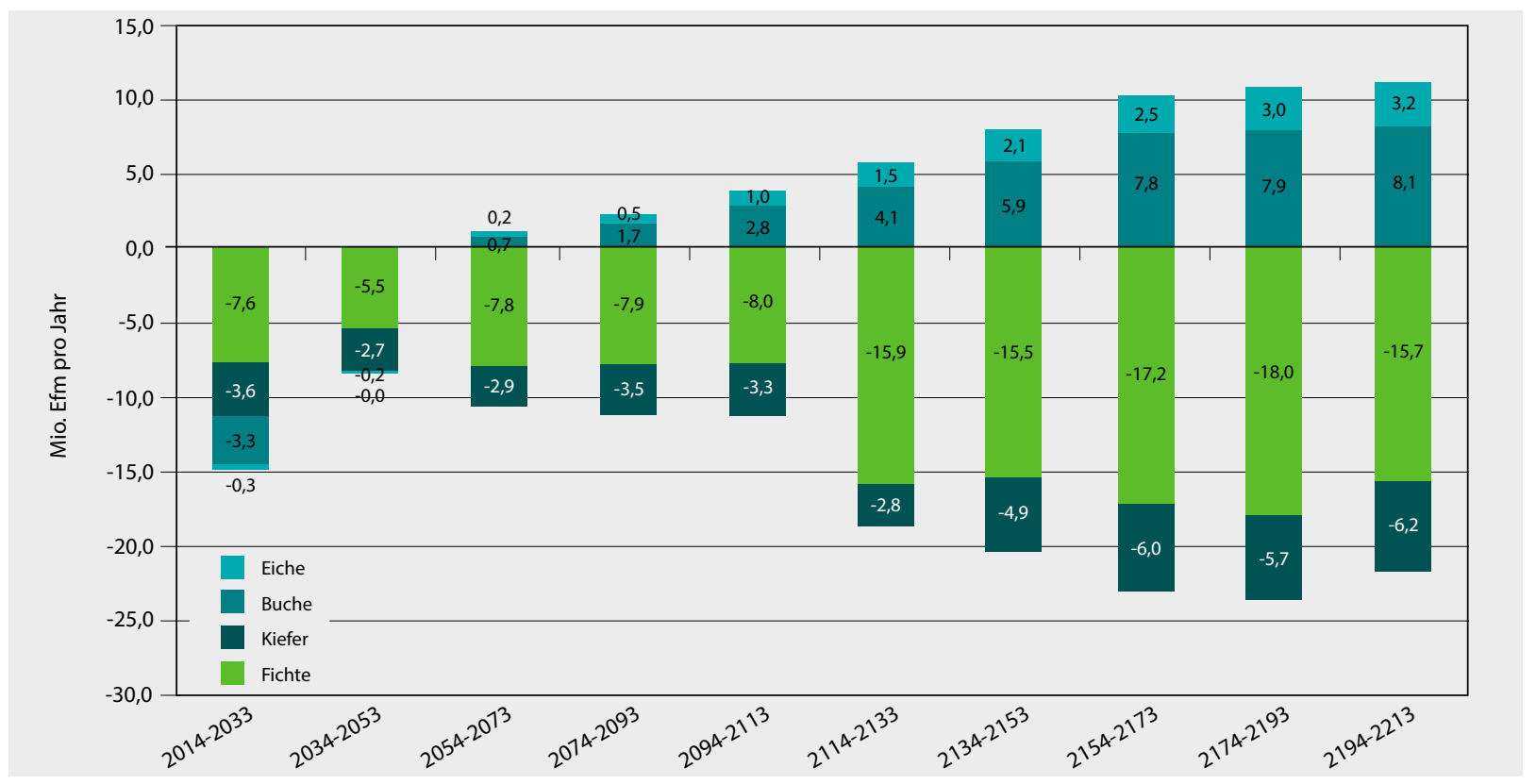

Abbildung 1

Differenzen des Rohholzaufkommens zwischen den Waldbehandlungsvarianten "Status quo" und „Neue Multifunktionalität" in Mio. Efm pro Jahr (Werte gerundet) 
Tabelle 3

Vergleich des minimalen, maximalen und mittleren Deckungsbeitrags in Euro pro Hektar und Jahr in der 200-jährigen Simulationsperiode für die Waldbehandlungsvarianten "Status quo" und "Neue Multifunktionalität" sowie die Gesamtdifferenz aus beiden Waldbehandlungsvarianten in Mrd. Euro je Jahr

\begin{tabular}{|c|c|c|c|}
\hline Deckungsbeitrag [€/ha/Jahr] & Mittelwert & Minimum & Maximum \\
\hline \multicolumn{4}{|l|}{ "Status quo" } \\
\hline Wald ohne forstliche Bewirtschaftung $(2,1 \%)$ & 0,0 & 0,0 & 0,0 \\
\hline Wald mit Naturschutzvorrangfunktion (8,5\%) & 178 & 158 & 194 \\
\hline Wirtschaftswald mit naturschutzfachlichen Mindeststandards $(89,4 \%)$ & 283 & 262 & 301 \\
\hline Status quo Gesamt & 269 & 249 & 285 \\
\hline \multicolumn{4}{|l|}{ „Neue Multifunktionalität“ } \\
\hline Wald ohne forstliche Bewirtschaftung (5\%) & 0,0 & 0,0 & 0,0 \\
\hline Wald mit Naturschutzvorrangfunktion (25\%) & 158 & 132 & 183 \\
\hline Wirtschaftswald mit naturschutzfachlichen Mindeststandards (70\%) & 193 & 180 & 217 \\
\hline Neue Multifunktionalität Gesamt & 174 & 163 & 195 \\
\hline 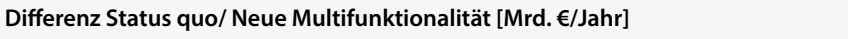 & $-1,0$ & $-1,1$ & $-0,8$ \\
\hline
\end{tabular}

Wird die Waldbehandlung im "Status quo" fortgeführt, so können im „Wirtschaftswald mit naturschutzfachlichen Mindeststandards" zwischen 262 und $301 € /$ ha/Jahr, im Mittel $283 € /$ ha/Jahr und auf den "Waldflächen mit Naturschutzvorrangfunktion" zwischen 158 bis $194 € /$ ha/Jahr, im Mittel $178 € /$ ha/Jahr an Deckungsbeitrag generiert werden. Insgesamt beläuft sich der Deckungsbeitrag, bezogen auf die deutsche Holzbodenfläche, auf rund 249 bis $285 € / \mathrm{ha} / \mathrm{Jahr}$ und im Mittel auf $269 € / \mathrm{ha} / \mathrm{Jahr}$. Dies entspricht bei einer Fortführung des "Status quo" einem Deckungsbeitrag zwischen 2,6 und 3,0 Mrd. $€ / J a h r$ bei einem Mittelwert von 2,9 Mrd. $€ / J a h r$.

In der Waldbehandlungsvariante "Neue Multifunktionalität“" reduzieren sich die Deckungsbeiträge der forstlichen Nutzung insgesamt im Mittel um rund $35 \%$ im Vergleich zum "Status quo". Im "Wirtschaftswald mit naturschutzfachlichen Mindeststandards" lassen sich nach Umsetzung der höheren naturschutzfachlichen Standards im Mittel $193 € /$ ha/Jahr (zwischen 180 und $217 € /$ ha/Jahr) an Deckungsbeitrag erzielen. Auf den „Waldflächen mit Naturschutzvorrangfunktion" liegt der Deckungsbeitrag bei rund $158 € /$ ha/Jahr mit Schwankungen zwischen 132 und $183 € /$ ha/Jahr. Bezogen auf die gesamte Holzbodenfläche errechnen sich bei einer Umsetzung der Waldbehandlungsvariante "Neuen Multifunktionalität" Deckungsbeiträge zwischen 163 und $195 €$ / ha/Jahr, im Mittel $174 €$ /ha/Jahr. Insgesamt können bei einer Umsetzung der „Neuen Multifunktionalität" zwischen 1,7 und 2,1 Mrd. €/Jahr, im Mittel 1,9 Mrd. €/Jahr generiert werden.

In der Differenz der beiden Waldbehandlungsvarianten ergibt sich ein Verlust an jährlichem Deckungsbeitrag (Opportunität) von 0,80 bis 1,1 Mrd. $€$, im Mittel von rund 1,0 Mrd. € pro Jahr.

In Abbildung 2 sind die Differenzen des Deckungsbeitrags in Mrd. Euro pro Jahr zwischen den Waldbehandlungsvarianten "Status quo" und "Neue Multifunktionalität" differenziert nach Holzartengruppen für den 200-jährigen Simulationszeitraum dargestellt. Die Differenzen der Deckungsbeiträge entstehen zu Beginn der Simulationsperiode primär aus den Aufwendungen für den Waldumbau (höhere Kulturkosten). Zu Ende der Simulationsperiode sind es primär die Verluste des ertragsstarken Nadelholzes, die zu den hohen Deckungsbeitragsdifferenzen führen. Zudem entstehen über die gesamte Simulationsperiode hinweg Deckungsbeitragsdifferenzen durch das verringerte Rohholzaufkommen, bedingt durch den Nutzungsverzicht für zusätzliche „Waldflächen ohne forstliche Bewirtschaftung", für das Belassen von Habitatbäumen und für Totholznachliefermengen sowie durch die Erhöhung der Holzerntekosten, bedingt durch Arbeitssicherheitsmaßnahmen und die Erweiterung der Rückegassen auf den „Wirtschaftswaldflächen mit naturschutzfachlichen Mindeststandards". Ebenfalls entstehen konstant über den gesamten Zeitraum hinweg zusätzliche Verwaltungskosten, die sich jedoch über die Fläche herleiten und nicht maßnahmenabhängig sind.

In unseren Berechnungen wird auch noch einmal deutlich, dass die Erhöhung des Einschlags in den Holzartengruppen Buche und Eiche (höherer Einschlag bewertet zu konstanten Preisen) den Rückgang des Einschlags in den Holzartengruppen Fichte und Kiefer (reduzierter Einschlag bewertet zu konstanten Preisen) hinsichtlich des erzielten Gesamtdeckungsbeitrags im 200-jährigen Simulationszeitraum nicht kompensieren kann. So werden die höheren Erlöse in den Holzartengruppen Buche und Eiche in der Waldbehandlungsvariante "Neue Multifunktionalität" durch den Waldumbau hin zu einem höheren Laubholzanteil durch die zusätzlichen Kulturkosten teilweise aufgehoben. Weiterhin weist die Holzartengruppe Buche nach TBN-Forst unter allen Holzartengruppen die niedrigsten durchschnittlichen Verkaufspreise pro Kubikmeter im Jahr 2011 auf. Im Vergleich zur ertragsstarken Holzartengruppe Fichte, mit rund $20 € / \mathrm{m}^{3}$ höheren durchschnittlichen Verkaufserlösen im Jahr 2011 


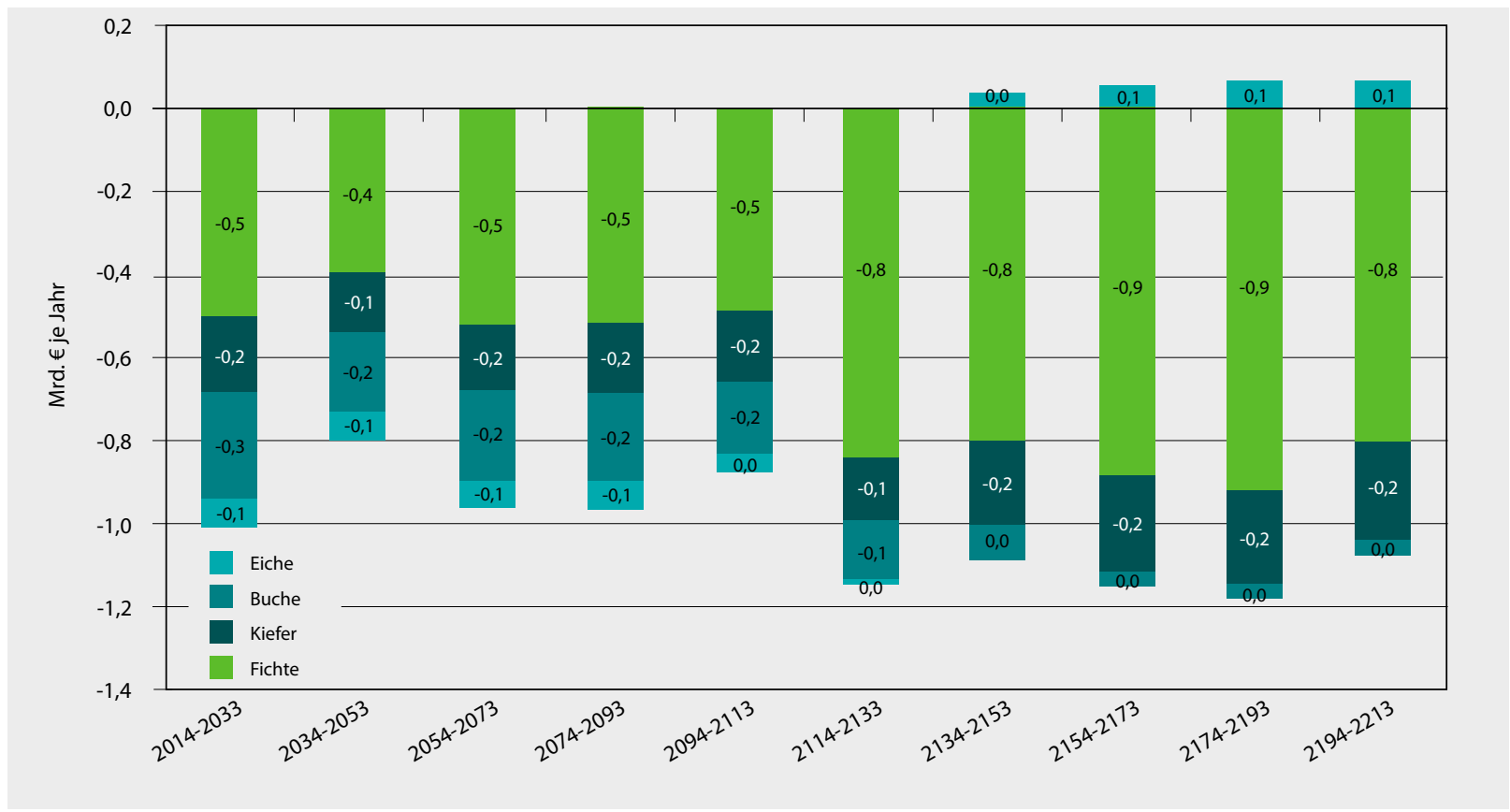

Abbildung 2

Differenzen des Deckungsbeitrags zwischen den Waldbehandlungsvarianten "Status quo" und "Neue Multifunktionalität" in Mrd. Euro pro Jahr (Werte gerundet)

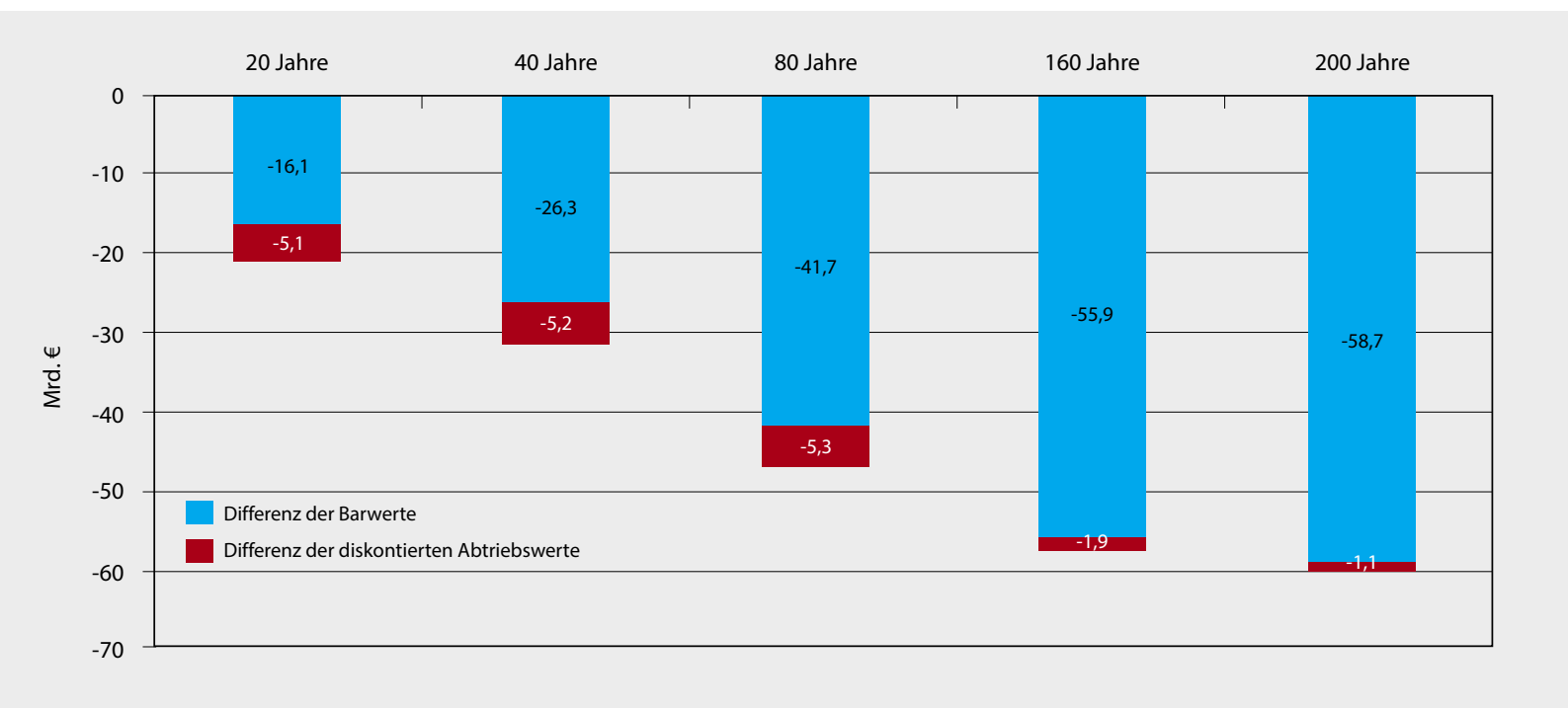

Abbildung 3:

Ertragswertdifferenzen (Barwerte und diskontierte Abtriebswerte) der Waldbehandlungsvarianten "Status quo" und "Neue Multifunktionalität“ in Mrd. Euro pro Jahr über verschiedenen Betrachtungszeiträume (Werte gerundet)

und deutlich höheren Einschlagsmengen, erzielt die Holzartengruppe Buche deutlich niedrigere Erlöse pro Hektar. Einen Sonderfall stellt die Holzartengruppe Eiche dar, welche unter allen Holzartengruppen die höchsten Verkaufspreise pro Kubikmeter im Jahr 2011 aufweist, jedoch auch nur geringe Einschlagsmengen ermöglicht (vgl. Ermisch et al., 2013).

\subsection{Ertragswert und Annuität}

Die Differenzen der Ertragswerte zwischen den beiden Waldbehandlungsvarianten, berechnet aus den Barwerten der Zahlungsströme und den diskontierten Abtriebswerten als Maß für die Wertänderung, sind in Abbildung 2 für verschiedene Bewertungszeiträume dargestellt.

Bezogen auf einen Bewertungszeitraum von 20 Jahren beträgt die Barwertdifferenz der Waldbehandlungsvariante "Neue Multifunktionalität" im Vergleich zum "Status quo" rund 16,1 Mrd. €. Die Differenz der diskontierten 


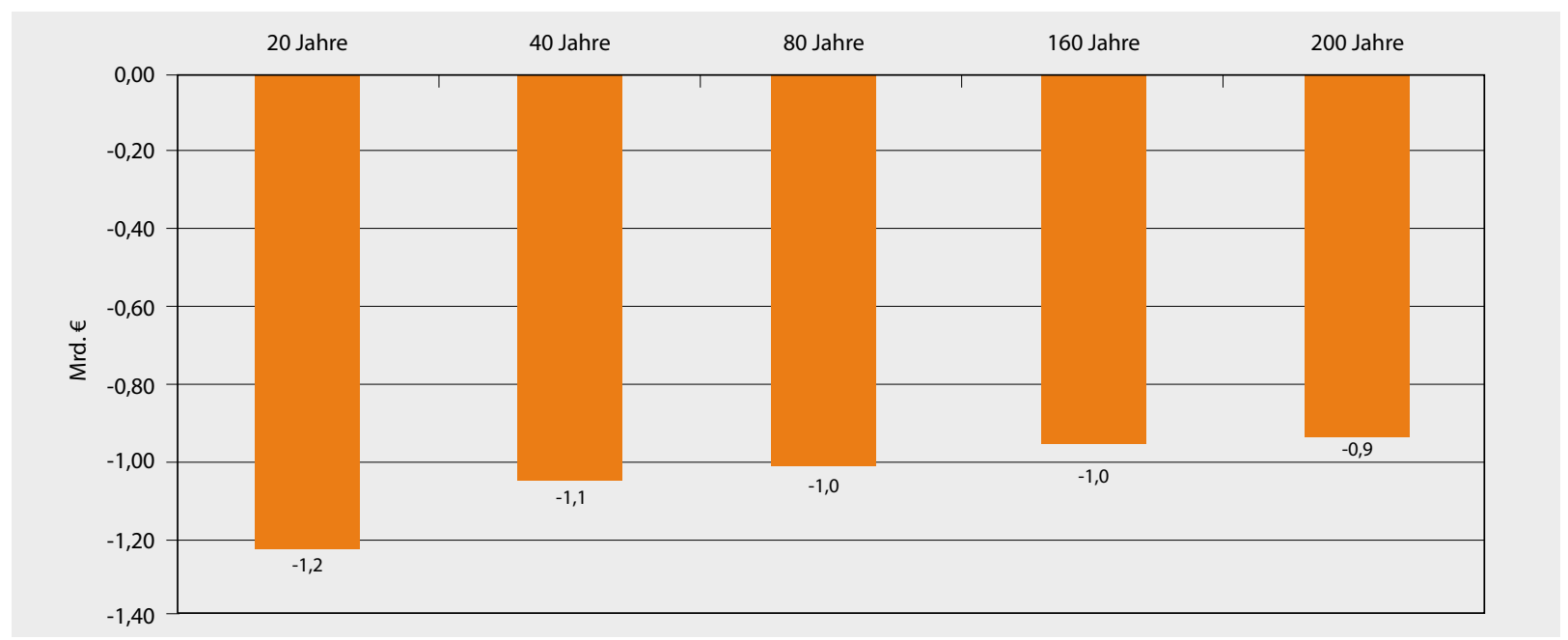

Abbildung 4

Annuitätendifferenzen der Waldbehandlungsvarianten "Status quo" und „Neue Multifunktionalität" in Mrd. Euro pro Jahr (Werte gerundet)

Abtriebswerte zwischen den beiden Waldbehandlungsvarianten beträgt rund 5,1 Mrd. $€$. Die statische (nicht diskontierte) Differenz der Abtriebswerte beträgt nach 20 Jahren rund 6,8 Mrd. €. Die Differenz des Ertragswerts beträgt rund 21,2 Mrd. €.

Bezogen auf einen Zeitraum von 200 Jahren beträgt die Barwertdifferenz rund 58,7 Mrd. $€$ und die Differenz der diskontierten Abtriebswerte rund 1,1 Mrd. €. Insgesamt beträgt die Ertragswertdifferenz rund 59,7 Mrd. $€$. Die nicht diskontierte Differenz der Abtriebswerte beträgt nach 200 Jahren rund 20,7 Mrd. $€$.

In einem nächsten Schritt wurden die Ertragswertdifferenzen in einen jährlich konstanten Zahlungsstrom überführt (vgl. Möhring et al., 2006). Beim Vergleich der Bewertungszeiträume von 20 und 40 Jahren sind die Annuitätendifferenzen zwischen dem "Status quo" und der "Neuen Multifunktionalität" noch deutlich zu sehen (Abbildung 4). Beim Vergleich der längeren Bewertungszeiträume gleichen sich diese aufgrund des Einflusses von Zins und Zeit immer mehr an. Berechnet für einen Zeitraum von 20 Jahren betragen die jährlichen Annuitätendifferenzen einschließlich der diskontierten Abtriebswerte rund 1,2 Mrd. €. Bezogen auf einen Zeitraum von 200 Jahren betragen die jährlichen Annuitätendifferenzen rund 0,9 Mrd. $€$.

\section{Diskussion und Schlussfolgerungen}

Mit der Studie sollten erstmalig orientierende Größenordnungen zu den Holzeinschlags-, Deckungsbeitrags- und Ertragswertverlusten der forstlichen Rohholzerzeugung bei einer Implementierung des naturschutzorientierten Waldbewirtschaftungskonzeptes "Neue Multifunktionalität" von Höltermann (2013) abgeschätzt werden. Hierfür wurde als eigener Ansatz eine Opportunitätskostenanalyse gewählt. Die naturschutzfachlichen Forderungen wurden auf Basis von Literaturrecherchen und eigenen Annahmen für die
Berechnung der Verzichtskosten operationalisiert und mit einer Waldbehandlungsvariante zur Fortführung der derzeitigen Waldbewirtschaftung verglichen. Bei den in dieser Studie getroffenen Annahmen handelt es sich um Durchschnittswerte für den gesamten deutschen Wald. Dies bedeutet jedoch nicht, dass die Maßnahmen so in der Realität in jeder Waldeigentumsart umgesetzt werden können.

Für die Berechnungen wurde das Strugholtz-EnglertSimulationsmodell eingesetzt. Hierbei handelt es sich um ein einfaches strategisches Entscheidungsmodell, mit dem die Auswirkungen unterschiedlicher Waldbehandlungsoptionen über einen Zeitraum von bis zu 200 Jahre berechnet werden können. Es wurde ursprünglich für Forstbetriebe entwickelt. Im Rahmen dieser Studie wurde es erstmalig auf Bundesebene ("Deutschlandbetrieb") angewendet. Durch den langen Simulationszeitraum ist das Modell besonders geeignet, um die Auswirkungen naturschutzfachlicher Maßnahmenplanungen zu berechnen. In die Zukunft gerichtete Simulationen, insbesondere mit derartig langen Betrachtungszeiträumen, unterliegen jedoch stets Unsicherheiten. So können sich neben Änderungen von Standorteignung und Wachstumsverläufen der Baumarten (z. B. durch Klimawandel) auch die gesellschaftlichen Zielsetzungen für die Waldbehandlung ändern. Des Weiteren basiert die Modellierung auf durchschnittlichen Eingangsdaten und konstanten Preisen und ist von den gewählten Setzungen abhängig. Die vorgestellten Ergebnisse können daher nur orientierende Größenordnungen bieten. In Bezug auf die Berücksichtigung von Risiko herrscht weiterer Modellentwicklungsbedarf.

Im Rahmen der Studie konnte auch die altersbedingte Holzqualitätsentwertung der Bäume (z. B. als Auswirkung der Forderung nach einer Erhöhung von Produktionszeiten zur Anhebung des Altholzanteils) sowie die damit verbundene Veränderung der Sortimentsstruktur aufgrund des geringen Kenntnisstandes nicht bewertet werden. Wenn forstpolitisch hohe Mindestproduktionszeiträume oder Zieldurchmesser vorgegeben werden, verlieren Forstbetriebe jedoch 
ihre Flexibilität, auf die am Markt nachgefragte Sortimentsstruktur sachgerecht zu reagieren und Holzentwertung zu vermeiden. Hinsichtlich der Berücksichtigung von Holzentwertung im verwendeten Modell besteht daher weiterer Forschungsbedarf.

Das berechnete Rohholzaufkommen bei einer Fortführung der Waldbewirtschaftung nach dem aktuellen Waldnaturschutzniveau („Status quo“) liegt mit 79,6 Mio. Efm/Jahr im Mittel des 200-jährigen Simulationszeitraumes grundsätzlich in einer plausiblen Größenordnung. Die tatsächlichen Nutzung zwischen der BWI 2002 und BWI 2012 beliefen sich auf 75,7 Mio. Efm/Jahr (BMEL, 2012). Durch das WEHAMBasisszenario 2002 wurde für die Simulationsperiode 2003 bis 2042 ein potenzielles Rohholzaufkommen von 78,2 Mio. Efm/Jahr ausgewiesen (BMELV, 2005b).

Bei einer Anhebung des Waldnaturschutzniveaus nach dem NMF-Bewirtschaftungskonzept würde sich das Rohholzaufkommen in den nächsten 200 Jahren um insgesamt 11,2 Mio. Efm/Jahr auf 68,4 Mio. Efm/Jahr reduzieren. Durch die NMF-Bewirtschaftung würden sich jedoch vor allem das forstwirtschaftlich bedeutsame Nadelholzaufkommen im 200-jährigen Mittel um 16,1 Mio. Efm/Jahr bzw. gegenüber der Bewirtschaftung nach dem "Status quo" um $32 \%$ reduzieren.

Die Forstwirtschaftliche Gesamtrechnung (FGR) weist für den Wirtschaftsbereich Forstwirtschaft in den 1990er Jahren überwiegend hohe Nettounternehmensverluste aus. Zu Beginn der 2000er-Jahre konnten ebenfalls keine Unternehmensgewinne erzielt werden (Seintsch und Rosenkranz, 2014). In Folge des deutlich gestiegenen Einschlages konnte der Wirtschaftsbereich Forstwirtschaft erstmalig ab dem Jahr 2005 Nettounternehmensgewinne erzielen. Im Jahr 2012 erzielte der Wirtschaftsbereich Forstwirtschaft mit einem Nettounternehmensgewinn von 1,3 Mrd. $€$ sein bisheriges Rekordergebnis. Zwei Drittel des Produktionswertes von 4,8 Mrd. $€$ zu Herstellungspreisen im Jahr 2012 resultierten hierbei aus der Erzeugung von Nadelrohholz (Seintsch und Rosenkranz, 2014). Bei einer Umsetzung des NMF-Bewirtschaftungskonzeptes, verbunden mit einem jährlichen Deckungsbeitragsverlust von 1,0 Mrd. € im 200-jährigen Mittel, würde eine aktuell erfolgreiche Branche ohne Kompensation wieder in die Verlustnähe gebracht werden. Wie Bormann und Dieter (2010) aufzeigen konnten, waren die Unternehmensgewinne des Wirtschaftsbereiches Forstwirtschaft unter den wirtschaftlichen Rahmenbedingungen der Jahre 1991 bis 2008 überwiegend von der Einschlagshöhe anhängig. Einschlagseinschränkungen, wie im Rahmen der Umsetzung der "Neuen Multifunktionalität" vorgesehen, wirken somit direkt auf die Gewinnhöhe und Leistungsfähigkeit der Forstwirtschaft, bspw. auch hinsichtlich der Bereitstellung unentgeltlicher Schutz- und Erholungsleistungen.

Durch die Berechnung von Ertragswert- und Annuitätendifferenzen für unterschiedliche Bewertungszeiträume wurde weiterhin ein Anhalt geboten, welcher Kompensationsbedarf den deutschen Forstbetrieben durch Opportunitätskosten in der Rohholzerzeugung bei einer Anhebung des Waldnaturschutzniveaus nach dem NMF-Konzept entsteht. So erzielten die staatlichen, körperschaftlichen und privaten Forstbetriebe des Testbetriebsnetzes Forstwirtschaft des BMEL (TBN-Forst) im Jahr 2013 bspw. 78 \% des Gesamtertrages durch Holzerlöse. Weiterhin verdeutlichen die Abtriebswertberechnungen, dass $u$. a. in Folge des naturschutzorientierten Waldumbaus der Wert des deutschen Waldes für künftige Generationen sinken würde, wenn die heutigen wirtschaftlichen Rahmenbedingungen unterstellt werden.

In Folge des reduzierten inländischen Rohholzaufkommens bei einer Umsetzung des NMF-Konzepts sind weiterhin Auswirkungen auf die holzbasierte Wertschöpfung und Beschäftigung in Deutschland zu erwarten. Im Jahr 2012 generierten die holzbasierten Wertschöpfungsketten des Clusters Forst und Holz mit 1,1 Mio. Gesamtbeschäftigten einen Umsatz 180,7 Mrd. € und eine Bruttowertschöpfung von $55 \mathrm{Mrd}$. $€$. Hiermit hatte der Cluster einen Anteil an der deutschen Volkswirtschaft von etwa 3,1 \% des Umsatzes und 3\% der Beschäftigung (Becher, 2014). Die inländische Rohholzverwendung insgesamt im Mittel der Jahre 2003 bis 2012 stütze sich bei einer Gesamtverwendung von jährlich 73,81 Mio. $\mathrm{m}^{3}$ zu 77,8 \% auf Nadelholz. Die stofflichen Verwender der 1. Verarbeitungsstufe mit der Säge-, der Holzwerkstoff- sowie der Holz- und Zellstoffindustrie waren mit einem Verwendungsanteil von 88,7 \% im besonderen Maße vom Nadelholz abhängig (Jochem et al., 2015). Seit dem Jahr 2010 ist die inländische Rohholzverwendung auf Nettoimporte beim Nadelrohholz angewiesen (Weimar, 2014). Das bei einer Umsetzung des NMF-Konzeptes deutlich reduzierte inländische Nadelrohholzaufkommen um 16,1 Mio. Efm/Jahr (200-jähriges Mittel) dürfte durch die 1. Verarbeitungsstufe der stofflichen Verwender durch zusätzliche Importe in dieser Größenordnung nicht kompensierbar sein, wie die Berechnungen von Dieter und Seintsch (2012) zur Wirkung eines Überseeimportszenarios für Nadelrohholz auf die Produktpreise verdeutlichen. In Folge der Umsetzung des NMFKonzeptes wären Kapazitätsanpassungen im Cluster Forst und Holz an das reduzierte inländische Rohholzaufkommen zu erwarten. Wie Dieter (2010) durch Input-Output-Analysen aufzeigte, würden aus einer reduzierten stofflichen Rohholzverwendung hohe volkswirtschaftliche Wertschöpfungsverluste resultieren. So entsteht durch die Weiterverarbeitung einer monetären Einheit Rohholz im Holzgewerbe ein Wertschöpfungseffekt mit dem Faktor 10,4. Unter der Annahme, dass im deutschen Holzgewerbe durch die NMF-Umsetzung jährlich 16,1 Mio. $\mathrm{m}^{3}$ Nadelrohholz mit einem Warenwert von 1,16 Mrd. € (TBN-Holzverkaufserlöse 2011) weniger verarbeitet würden, wären Wertschöpfungsverluste von jährlich rund $12 \mathrm{Mrd} . €$ verbunden. In Folge einer reduzierten holzbasierten Wertschöpfung wären zudem fiskalische Effekte zu berücksichtigen. Nach Dieter und Bormann (2009) ergeben sich aus der Weiterverarbeitung einer zusätzlichen Einheit Rohholz mit einem Wert von 1 Mio. $€$ als direkte fiskalische Effekte (nur Forstwirtschaft und Holzgewerbe) Steuereinnahmen und Sozialversicherungsbeiträge in Höhe von 1,43 Mio. $€$ und indirekte fiskalische Effekte (gesamte Volkswirtschaft) von 4,01 Mio. $€$.

Die vorliegende Studie basiert überwiegend auf Annahmen. Ob und in welchem Maß das Waldnaturschutzniveau in 
Zukunft angehoben wird, ist bisher nicht bekannt. Bislang wird der im Allgemeinen stärker gemeinwohlorientierte öffentliche Wald in höherem Maße bei der Umsetzung naturschutzfachlicher Maßnahmen in die Pflicht genommen als der Privatwald. Ob die Naturschutzmaßnahmen in der hier dargestellten Form überhaupt auf den Privatwald übertragen werden können ist fraglich. Bei einer Umsetzung von Naturschutzmaßnahmen auf der gesamten Waldfläche muss zudem die steigende Nachfrage nach Rohholz zur energetischen und stofflichen Nutzung in den Abwägungsprozess miteinbezogen werden. Denkbar wäre hier beispielsweise eine Steigerung des Waldnaturschutzniveaus im öffentlichen Wald, verbunden mit einer Einschlagssteigerung zum Ausgleich im Privatwald.

Die Umsetzung der "Neue Multifunktionalität" von Höltermann (2013) zur Anhebung des Naturschutzniveaus im deutschen Wald wäre eine rationale Entscheidung, wenn der zusätzliche Nutzen an Naturschutzleistungen bei der Implementierung zumindest in Höhe der Opportunitätskosten der forstlichen Nutzung (Rohholzproduktion), wenn nicht sogar in Höhe der verminderten Wertschöpfung der nachgelagerten Holzindustrie und der fiskalischen Effekte liegen würde. Es ist derzeit jedoch noch unbekannt, wann dieser Nutzen auf den "Waldflächen ohne forstliche Bewirtschaftung" und „Waldflächen mit Naturschutzvorrangfunktion" in der Zukunft entsteht. Denkbar ist, dass bspw. die natürliche Waldentwicklung hin zu "Urwäldern von morgen“ ihren vollen Nutzen erst nach dem gewählten Betrachtungszeitraum entfaltet.

\section{Danksagung}

Wir danken Dr. Markus Dög, Nils Ermisch und Claudia-Christin Krüger für ihre Unterstützung bei den Korrekturen und bei der Festlegung der Steuergrößen und Hermann Englert für die Unterstützung bei den Berechnungen. Weiterhin danken wir Prof. Dr. Matthias Dieter sowie den beiden anonymen Gutachtern für die hilfreichen Anmerkungen und Verbesserungsvorschläge.

\section{Literatur}

AFL Niedersachsen (2010) Richtpreise, Tarife, Kalkulationen, Adressen. Wiegersen : Arbeitsgemeinschaft forstwirtschaftlicher Lohnunternehmer, AFL-Info 2010/10

BaySF - Bayern / Staatsforsten (2009) Naturschutzkonzept der Bayerischen Staatsforsten [online]. Zu finden in <http://www.baysf.de/fileadmin/ user_upload/03-wald_schuetzen/pdf/Naturschutzkonzept_Bayerische_ Staatsforsten.pdf $>$ [zitiert am 18.11.2015]

Becher G (2014) Clusterstatistik Forst und Holz : Tabellen für das Bundesgebiet und die Länder 2000 bis 2012 . Hamburg : Johann Heinrich von Thünen-Institut, 105 p, Thünen Working Paper 32, DOI:10.3220/ WP_32_2014

BfN (2012) Waldlebensraumtypen in Deutschland: Sonderauswertung des BfN im Auftrag des Verbundforschungsprojektes FFH-Impact. (Stand: Mai 2012). Bonn : Bundesamt für Naturschutz

BMELV - Bundesministerium für Ernährung, Landwirtschaft und Verbraucherschutz (2005a) Das Waldentwicklungsmodell 2003 bis 2042 :
Modell und Ergebnisse zu den Bundeswaldinventur-Erhebungen 2001 bis 2002 und 1986 bis 1988. Berlin : BMELV, $99 \mathrm{p}$

BMELV - Bundesministerium für Ernährung, Landwirtschaft und Verbraucherschutz (2005b) Das potenzielle Rohholzaufkommen 2003 bis 2042 : zu den Bundeswaldinventur-Erhebungen 2001 bis 2002 und 1986 bis 1988 : Teil 2: Tabellen und Methode. Berlin : BMELV

BMELV - Bundesministerium für Ernährung, Landwirtschaft und Verbraucherschutz (2011) Waldstrategie 2020 : nachhaltige Waldbewirtschaftung - eine gesellschaftliche Chance und Herausforderung. Berlin : BMELV, $34 \mathrm{p}$

BMEL - Bundesministerium für Ernährung und Landwirtschaft (2012) Der Wald in Deutschland : ausgewählte Ergebnisse der 3. Bundeswaldinventur (online]. Zu finden in <http://www.bmel.de/SharedDocs/Downloads/ Broschueren/Bundeswaldinventur3.pdf?_blob=publicationFile $>$ [zitiert am 18.11.2015]

BMU - Bundesministerium für Umwelt, Naturschutz und Reaktorsicherheit (2007) Nationale Strategie zur biologischen Vielfalt : vom Bundeskabinett am 07. November 2007 beschlossen. Berlin : BMU, $178 p$

BNatSchG (2010) Bundesnaturschutzgesetz vom 29. Juli 2009 (BGBI. I S. 2542), das zuletzt durch Artikel 421 der Verordnung vom 31. August 2015 (BGBI. I S. 1474) geändert worden ist [online]. Zu finden in <http://www.gesetze-im-internet.de/bundesrecht/bnatschg_2009/gesamt.pdf> [zitiert am 08.12.2015]

Bormann K, Dieter M (2010) Was bedeutet der Einschlag für den Gewinn der Forstbetriebe? AFZ Wald 65(23):5-7

Dieter M (2010) Produktion, Weiterverarbeitung und Handel von Holz aus gesamtwirtschaftlicher Perspektive : eine quantitative Analyse der Forst- und Holzwirtschaft auf Grundlage sektoraler Statistiken. Göttingen : Univ, $64 \mathrm{p}$

Dieter M, Bormann K (2009) Fiskalische Effekte von Holznutzung im intersektoralen Vergleich. Allg Forst Jagdzeitg 180(7/8):170-175

Dieter M, Elsasser P, Küppers J-G, Seintsch B (2008) Rahmenbedingungen und Grundlagen für eine Strategie zur Integration von Naturschutzanforderungen in die Forstwirtschaft. Hamburg : vTI, 36 , Arbeitsber Inst Ökon Forst Holzwirtsch vTI 2008/02

Dieter M, Seintsch B (2012) Änderung der Wettbewerbsfähigkeit der Holzund Papierwirtschaft in Deutschland auf Grund zunehmender Knappheit an Nadelholz. Allg Forst Jagdzeitg 183(5/6):116-128

Engel F (2013) Bilanz der bundesweiten Flächen mit natürlicher Waldentwicklung: F+E-Vorhaben NWE5 „Natürliche Waldentwicklung als Ziel der Nationalen Strategie zur biologischen Vielfalt" [online]. Zu finden in <http://www.nw-fva.de/nwe5/downloads/Bilanzierung.pdf> [zitiert am 18.11.2015]

Ermisch N, Seintsch B, Dieter M (2013) Holzartengruppe Fichte 2003 bis 2011 konstant mit höchstem Erlösbeitrag. AFZ Wald 68(23):6-9

Forests Europe - Ministerial Conf Protection (2011) State of Europe's forests 2011 : status and trends in sustainable forest management in Europe. Oslo : Ministerial Conf Protection Forests Europe, $337 \mathrm{p}$

Forst Brandenburg - Brandenburg / Ministerium Landwirtschaft Umweltschutz Raumordnung (2004) Waldbau-Richtlinie 2004 : "Grüner Ordner" der Landesforstverwaltung Brandenburg. Potsdam: Minist Landwirtsch Umweltschutz Raumordnung, $143 \mathrm{p}$

FSC - Forest Stewartship Council (2012) Deutscher FSC-Standard : Version 2.3 vom 01.07.2012. Freiburg: FSC, $51 \mathrm{p}$

FVA (2007) Forstliche Nutzung in FFH-Gebieten : Situationsanalyse und Perspektiven ; Fachstudie erstellt durch die Forstliche Versuchs- und Forschungsanstalt Baden-Württemberg. Freiburg: FVA, $86 \mathrm{p}$

HessenForst (2008) Hessische Waldbaufibel : Grundsätze und Leitlinien zur naturnahen Wirtschaftsweise im hessischen Staatswald. Kassel : Hessen-Forst, $98 \mathrm{p}$

Hessen-Forst (2011) Arten- und Biotopschutz im Hessischen Staatswald: Die Naturschutzleitlinie im Kurzporträt. Landesbetrieb Hessen-Forst, Kassel, $25 \mathrm{~S}$

Höltermann A (2013) Das 5\%-Ziel : Begründung und Bedeutung. Holz Zentralbl 139(40):977-978

Jochem D, Weimar H, Bösch M, Mantau U, Dieter M (2015) Estimation of wood removals and fellings in Germany : a calculation approach based on the amount of used roundwood. Eur J Forest Res 134(5):869-888 
Küppers J-G, Dieter M (2008) Belastungen der Forstbetriebe aus der Schutzund Erholungsfunktion des Waldes (2003 bis 2006). Hamburg : vTI, 29 p, Arbeitsber Inst Ökon Forst Holzwirtsch vTI 2008/04

Landesforst Mecklenburg-Vorpommern (1996) Ziele und Grundsätze einer naturnahen Forstwirtschaft in Mecklenburg- Vorpommern. Wald und Forstwirtschaft in Mecklenburg-Vorpommern 4(SH Jan):13-20

Landesforst Mecklenburg-Vorpommern (2002) Richtlinie zur Sicherung von Alt- und Totholzanteilen im Wirtschaftswald : mit erläuternder Einführung. Schwerin : Minist, $16 \mathrm{p}$

Landesforstbetrieb Sachsen Anhalt / Ministerium für Landwirtschaft und Umwelt (2014) Leitlinie Wald 2014 : zur Erhaltung und weiteren nachhaltigen Entwicklung des Waldes im Land Sachsen-Anhalt. Magdeburg: Minist, $54 \mathrm{p}$

Landesforsten Rheinland-Pfalz / Ministerium für Umwelt, Landwirtschaft, Weinbau und Forsten (2011) BAT-Konzept : Konzept zum Umgang mit Biotopbäumen, Altbäumen und Totholz bei Landesforsten Rheinland-Pfalz. Mainz : Minist, $25 \mathrm{p}$

Meyer P, Menke N, Nagel J, Hansen J, Kawaletz H, Paar U, Evers J (2009) Entwicklung eines Managementmoduls für Totholz im Forstbetrieb : Abschlussbericht des von der Deutschen Bundesstiftung Umwelt geförderten Projekts. Göttingen : Nordwestdtsche Forstl Versuchsanst, $110 \mathrm{p}$

Möhring B, Rüping U (2006) Bewertungskonzept für forstliche Nutzungsbeschränkungen. Frankfurt a M : Sauerländer, 48 p, Schr Forstökon 32

Möhring B, Rüping U, Leefken G, Ziegeler M (2006) Die Annuität - ein „missing link" der Forstökonomie?. Allg Forst Jagdzeitg 177(2):22-29

Niedersächsische Landesforsten (2011) Das LÖWE-Programm : 20 Jahre langfristige ökologische Waldentwicklung. Braunschweig : Nieders Landesforsten, $31 \mathrm{p}$

Oesten G, Roeder A (2002) Management von Forstbetrieben : Bd I: Grundlagen, Betriebspolitik. Remagen : Kessel, $364 \mathrm{p}$

PEFC (2009) PEFC-Standards für Deutschland : Leitlinie für nachhaltige Waldbewirtschaftung zur Einbindung des Waldbesitzers in den regionalen Rahmen [online]. Zu finden in <http://www.afl-hessen.de/dokumente/ pefc_Standards_01012011.pdf> [zitiert am 18.11.2015]

Polley H (2009) Wald in Schutzgebieten : ein Überblick. Landbauforsch SH 327:75-82

Posavec S, Beljan K, Lovric M (2011) Model of compensation payment to the owners on Natura 2000 forest sites [online]. Zu finden in <https://bib.irb. hr/datoteka/591569.glasnikSP_1-2011_sparat.pdf> [zitiert am 19.11.2015]

Rosenkranz L, Wippel B, Seintsch B (2012) FFH-Impact : Teil 1: Umsetzung der FFH-Richtlinie im Wald in den Bundesländern. Hamburg : vTI, 115 , Arbeitsber Inst Ökon Forst Holzwirtsch vTI 2012/04

Rosenkranz L, Seintsch B, Wippel B, Dieter M (2014) Income losses due to the implementation of the Habitats Directive in forests : conclusions from a case study in Germany. Forest Pol Econ (38):207-218, doi:10.1016/j.forpol.2013.10.005

SaarForst (2003) Das „Dicke Buchen-Programm“ des SaarForst Landesbetriebs zum Schutz von Höhlenbäumen [online]. Zu finden in <http://www.saarforst-saarland.de/images/stories/Microsoft\%20Word\%20-\%20Dicke\%20 Buchen-Programm-2.doc.pdf> [zitiert am 20.11.2015]

Schmack S, Aichholz R, Hartebrodt C (2013) Naturschutzfachliche Einschränkungen der Forstwirtschaft : ökonomische Katastrophe oder Bagatelle? Freiburg : FVA, 103 p, Ber Freiburger Forstl Forsch 97

Schmalfuß N, Lorho F, Braun W (2010) Alt- und Totholzkonzept BadenWürttemberg. Stuttgart : ForstBW, $36 \mathrm{p}$

Seintsch B, Rosenkranz L (2014) Erneutes Rekordergebnis der Forstwirtschaft : auch ein Grund zur Nachdenklichkeit? - Ergebnisse der Forstwirtschaftlichen Gesamtrechnung 2012. Holz-Zentralbl 140(13):316-317

Seintsch B, Rosenkranz L, Englert H, Dieter M, Wippel B, Becker G, Stratmann J Gerst J, Möhring B (2012) FFH-Impact : Teil 2: Auswirkungen von FFH-Maßnahmenplanungen auf Forstbetriebe. Hamburg : vTI, 144 , Arbeitsber Inst Ökon Forst Holzwirtsch 2012/05

Smaltschinski T (2001) Regionale Waldwachstumsprognose. Freiburg : Forstl Versuchs Forschungsanst Baden-Württemberg, 121 p, SchrR Freiburger Forstl Forsch 12
Strugholtz A (2010) Ein forstbetriebliches Simulationsmodell zur ökonomischen Bewertung strategischer forstlicher Produktionsentscheidungen. Göttingen : Univ, $77 \mathrm{p}$

Thüringen-Forst (Jahr unbekannt) Thöne K-F, Habitatbaum- und Totholzkonzept für den Staatswald des Freistaates Thüringen [online]. Zu finden in <http://www.waldbesitzerportal.de/imperia/md/content/waldbesitzerportal/habitatbaumkonzept.pdf> [zitiert am 19.11.2015]

Wagner S, Jönsson A (2001) Einschränkungen der Waldbewirtschaftung durch Naturschutzauflagen am Beispiel des europäischen Schutzgebietssystems Natura 2000 : Rechtsfragen und monetäre Bewertung ; Gutachten im Auftrag der Arbeitsgemeinschaft Deutscher Waldbesitzerverbände [online]. Zu finden in <http://joensson.net/doc/Gutachten-Endfassung.pdf < [zitiert am 19.11.2015]

Weimar H (2014) Holzbilanzen 2012 und 2013 für die Bundesrepublik Deutschland. Hamburg : Johann Heinrich von Thünen-Institut, 37 p, Thünen Working Paper 31, DOI:10.3220/WP_31_2014

Wildmann S (2013) Ökonomische Bewertung der bestehenden Flächen mit natürlicher Waldentwicklung : F+E-Vorhaben NWE5 "Natürliche Waldentwicklung als Ziel der Nationalen Strategie zur biologischen Vielfalt" [online]. Zu finden in <http://www.nw-fva.de/nwe5/downloads/ Oekonomische_Bewertung.pdf $>$ [zitiert am 19.11.2015]

Winkel G, Volz K-R (2003) Naturschutz und Forstwirtschaft : Kriterienkatalog zur "Guten fachlichen Praxis" ; Ergebnisse aus dem F+E-Vorhaben FKZ 80084001 des Bundesamtes für Naturschutz. Bonn : BfN, 187 p, Angew Landschaftsökol 52

Winter S (2010) Wie viel Totholz braucht Natur? : Symposium „Mehr Holz im Einklang mit der Gesellschaft" [online]. Zu finden in <http://veranstaltungen.fnr.de/fileadmin/allgemein/pdf/veranstaltungen/waldstrategie/2010/Winter.pdf> [zitiert am 19.11.2015] 

3. SEINTSCH B und ROSENKRANZ L (2015A): Wertschöpfung und Beschäftigung durch Holznutzung : Abschätzung der holzbasierten Wertschöpfung und Beschäftigung in stofflichen Wertschöpfungsketten. Holz-Zentralblatt 141(5):110-112 
Abschätzung der holzbasierten Wertschöpfung und Beschäftigung in stofflichen Wertschöpfungsketten

Björn Seintsch und Lydia Rosenkranz

Seit der Jahrtausendwende haben sich die deutschen Rohholzmärkte sehr dynamisch entwickelt. Der Einschlag der Forstwirtschaft, die stoffliche und energetische Holzverwendung und die Rohholzpreise sind deutlich gestiegen. Die energetische Nutzung in Privathaushalten ist zu einem der mengenbedeutendsten Holzverwendungsbereiche aufgestiegen. Wie die Bundeswaldinventur 2012 aufgezeigt hat, sind die Nutzungspotenziale der wirtschaftlich bedeutsamen Fichte abgeschöpft. Ebenso wurde die forstliche Rohholzproduktion auf Teilflächen des Waldes zur Verwirklichung naturschutzpolitischer Ziel extensiviert oder eingestellt. Aufgrund dieser Entwicklung sind die Nutzungskonkurrenzen um Waldflächen und Rohholz deutlich gestiegen. Die Frage nach der Wertschöpfung und Beschäftigung der unterschiedlichen Waldflächen- und Rohholznutzungsalternativen gewinnt zunehmend an Bedeutung. Vor diesem Hintergrund soll nachfolgend ein Ansatz zur Abschätzung der holzbasierten Wertschöpfung und Beschäftigung in stofflichen Wertschöpfungsketten vorgestellt werden.

Mit dem nachfolgenden Beitrag soll zunächst die Stellung der holzbasierten Vorleistungen in der Kostenstruktur ausgewählter Wirtschaftszweige der ersten und zweiten Verarbeitungsstufe in den Jahren 2006 und 2010 aufgezeigt werden. Hierauf aufbauend wird ein Ansatz zur Abschätzung der holzbasierten Wertschöpfung und Beschäftigung entlang unterschiedlicher stofflicher Wertschöpfungsketten vorgestellt. Diesen und anderen Fragestellungen widmete sich das Thünen-Institut im Rahmen des vom $\mathrm{BMBF}^{1}$ geförderten Verbundprojekts „Bioenergie-Regionen stärken (BEST)“. Als Vorbemerkung ist voranzustellen, dass nachfolgend Kennzahlen zum Bruttoproduktionswert sowie zur Bruttowertschöpfung und Beschäftigung der Kostenstrukturstatistik (StBA: F 4, R 4.3) vorgestellt werden, welche von den Angaben der Clusterstatistik Forst und Holz des Thünen-Instituts abweichen (Seintsch 2013; Becher 2014). Mit der eigenen Darstellung soll ein konsistentes Bild der verwendeten Datenquellen zur Abschätzung der holzbasierten Wertschöpfung und Beschäftigung in stofflichen Wertschöpfungsketten geboten werden. Während die Kostenstrukturstatistik mit ihrer Erfassungsgrenze von Unternehmen ab 20 Beschäftigten kleinere Wirtschaftseinheiten nicht berücksichtigt, sind diese durch die Datenquellen der Clusterstatistik Forst und Holz erfasst.

\section{Datengrundlage}

Als Datengrundlage für die Analysen wurden die Kostenstrukturstatistik (StBA: F 4, R 4.3) sowie die Material- und Wareneingangsstatistik (StBA: F 4, R 4.2.4) des Statistischen Bundesamtes verwendet. In beiden Statistiken werden Unternehmen ab 20 Beschäftigten nach dem Schwerpunktprinzip in der „Klassifikation der Wirtschaftszweige (WZ)“ erfasst. Dies bedeutet, dass die Unternehmen anhand der Haupttätigkeit mit dem größten Wertschöpfungsanteil zugeordnet werden.

\footnotetext{
${ }^{1}$ Die Verantwortung für den Inhalt dieser Veröffentlichung liegt beim Autor.
} 
Die jährlich erscheinende Kostenstrukturstatistik des Verarbeitenden Gewerbes (StBA: F 4, R 4.3) liefert Informationen über den Bruttoproduktionswert, die Bruttowertschöpfung, den Bruttoproduktionsgewinn (d.h. Unternehmereinkommen und Grundrente für den Boden) und die Beschäftigung der Unternehmen des Holz- und Papiergewerbes. Die Vorleistungen sind in der Kostenstrukturstatistik jedoch nicht hinreichend differenziert, um Aussagen zu den eingesetzten holzbasierten Rohstoffen, Halb- und Fertigwaren treffen zu können. Die vierjährlich erscheinende Material- und Wareneingangsstatistik des Verarbeitenden Gewerbes (StBA: F 4, R 4.2.4) liefert hingegen Informationen über die warenmäßige Zusammensetzung der Vorleistungen zu Anschaffungskosten. In dieser Statistik sind die bezogenen Waren nach der Systematik des „Warenverzeichnisses für die Material- und Wareneingangserhebung (WE)“ in Warengruppen zusammengefasst, welches auf dem „Güterverzeichnis für Produktionsstatistiken (GP)“ beruht. Für die nachfolgenden Darstellungen wurden diese tiefer untergliederten Warengruppen der Material- und Wareneingangsstatistik zu den Gruppen Rohholz, Holzhalbwaren, Holzfertigwaren, Papierhalbwaren, Papierfertigwaren und sonstige Vorleistungen zusammengefasst. Die Gruppe Papierfertigwaren wurde jedoch von keinem der hier dargestellten Wirtschaftszweige in den betrachteten Jahren als Vorleistung bezogen. Diese bezogenen Warengruppen werden von den holzwirtschaftlichen Unternehmen zur Gütererzeugung und als Handelsware eingesetzt. Ebenso wurden die einzelnen Positionen der Kostenstrukturstatistik aus Darstellungsgründen zusammengefasst. Durch die Verknüpfung beider Statistiken wird es möglich, den Anteil holzbasierter Vorleistungen in der Kostenstruktur der Unternehmen zu bestimmen (Seintsch 2011). Darüber hinaus können auf dieser Datenbasis holzbasierte Wertschöpfungsketten abgebildet werden.

\section{Rohholz im Wareneingang des Verarbeitenden Gewerbes}

Die Material- und Wareneingangsstatistik bietet einen Überblick zum beschafften Rohholz innerhalb des Verarbeitenden Gewerbes. In den beiden aktuellsten Ausgaben der Statistik wird für das Verarbeitende Gewerbe ein Wert des bezogenen Rohholzes von insgesamt 2,8 Mrd. € im Jahr 2006 und 3,5 Mrd. € im Jahr 2010 ausgewiesen. Aus Tabelle 1 wird ersichtlich, dass insgesamt zwölf Wirtschaftszweige Rohholz als Vorleistung bezogen haben. In der ersten Verarbeitungsstufe sind dies die Säge-, die Holzwerkstoff- und die Holz- und Zellstoffindustrie und in der zweiten Verarbeitungsstufe die Holzverpackungs-, die Holzbau-, die Möbel- und die Papierindustrie (Eine Umschlüsselung der Wirtschaftszweige von der im Berichtsjahr 2006 gültigen WZ 2003 nach der gültigen WZ 2008 für das Berichtsjahr 2010 findet sich bspw. in Seintsch 2013).

Mit Ausnahme der Holzverpackungsindustrie ist in allen Wirtschaftszweigen der Wert des beschafften Rohholzes zwischen 2006 und 2010 gestiegen. Vom gesamten Rohholzbezug des Verarbeitenden Gewerbes im Jahr 2010 entfällt mit 54 \% bzw. 1,9 Mrd. € erwartungsgemäß der höchste Anteil auf die Sägeindustrie. An zweiter Stelle folgt die Holzwerkstoffindustrie mit 684 Mio. $€$. Der Wert des bezogenen Rohholzes in der Papierindustrie belief sich auf 327 Mio. $€$ und bei den Holz- und Zellstoffherstellern auf 247 Mio. $€$ im Jahr 2010. 
Bei den weiteren Wirtschaftszweigen in Tabelle 1 ist der absolute Wert des bezogenen Rohholzes sowie dessen Anteil am Rohholzbezug des Verarbeitenden Gewerbes deutlich geringer. Die Materialund Wareneingangsstatistik liefert hiermit zugleich einen möglichen Erklärungsbeitrag, weshalb bei Unternehmen ab 20 Beschäftigten Unschärfen zwischen den Ergebnissen empirischer (funktionaler) Erhebungen (z.B. Döring und Mantau (2012) zur Anzahl der Schnittholzerzeuger) und amtlichen Statistiken nach dem Schwerpunktprinzip auftreten können. Nicht sämtliche Unternehmen mit Schnittholzerzeugung dürften bspw. aufgrund des Schwerpunktprinzips in den amtlichen Statistiken dem Wirtschaftszweig „1610 Säge-, Hobel- und Holzimprägnierwerke“ zugeordnet sein.

Tabelle 1: Warengruppe Rohholz als Vorleistung im Verarbeitenden Gewerbe

\begin{tabular}{|c|c|c|c|c|c|}
\hline \multirow[t]{2}{*}{$\mathbf{W Z}$} & \multirow[t]{2}{*}{ Bezeichnung } & \multicolumn{2}{|c|}{$\begin{array}{c}\text { Bezogenes Rohholz des } \\
\text { Wirtschaftszweiges } \\
\text { [Tsd. } € \text { ] }\end{array}$} & \multicolumn{2}{|c|}{$\begin{array}{c}\text { Anteil des Rohholzbezuges } \\
\text { des Wirtschaftszweiges am } \\
\text { Verarbeitenden Gewerbe } \\
{[\%]}\end{array}$} \\
\hline & & 2006 & 2010 & 2006 & 2010 \\
\hline (1) & (2) & (3) & (4) & (5) & (6) \\
\hline 1610 & $\begin{array}{l}\text { Säge-, Hobel- und Holzimpräg- } \\
\text { nierwerke }\end{array}$ & 1.731 .243 & 1.883 .046 & 61,8 & 53,8 \\
\hline 1621 & $\begin{array}{l}\text { Herstellung von Furnier-, Sper- } \\
\text { rholz-, Holzfaser- und Holz- } \\
\text { spanplatten }\end{array}$ & 365.392 & 684.152 & 13,0 & 19,6 \\
\hline 1622 & Herstellung von Parketttafeln & -- & 9.020 & -- & 0,3 \\
\hline 1623 & $\begin{array}{l}\text { Herstellung von sonstigen } \\
\text { Konstruktionsteilen, Fertigbau- } \\
\text { teilen, Ausbauelementen und } \\
\text { Fertigteilbauten aus Holz }\end{array}$ & 127.848 & 127.602 & 4,6 & 3,6 \\
\hline 1624 & $\begin{array}{l}\text { Herstellung von Verpackungs- } \\
\text { mitteln, Lagerbehältern und } \\
\text { Ladungsträgern aus Holz }\end{array}$ & 77.930 & 63.665 & 2,8 & 1,8 \\
\hline 1629 & $\begin{array}{l}\text { Herstellung von Holzwaren a. n. } \\
\text { g., Kork-, Flecht- und Korbwa- } \\
\text { ren (ohne Möbel) }\end{array}$ & 35.087 & 111.573 & 1,3 & 3,2 \\
\hline 1711 & $\begin{array}{l}\text { Herstellung von Holz- und } \\
\text { Zellstoff }\end{array}$ & 178.568 & 247.044 & 6,4 & 7,1 \\
\hline 1712 & $\begin{array}{l}\text { Herstellung von Papier, Karton } \\
\text { und Pappe }\end{array}$ & 269.066 & 327.375 & 9,6 & 9,4 \\
\hline 3101 & $\begin{array}{l}\text { Herstellung von Büro- und } \\
\text { Ladenmöbeln }\end{array}$ & 2.262 & 8.704 & 0,1 & 0,2 \\
\hline 3102 & Herstellung von Küchenmöbeln & k. A. & 2.226 & k. A. & 0,1 \\
\hline 3103 & Herstellung von Matratzen & k. A. & 8.371 & k. A. & 0,2 \\
\hline 3109 & $\begin{array}{l}\text { Herstellung von sonstigen } \\
\text { Möbeln }\end{array}$ & 9.169 & 24.195 & 0,3 & 0,7 \\
\hline & Summe & 2.796 .565 & 3.496 .973 & 100 & 100 \\
\hline
\end{tabular}

\section{Kostenstruktur und Beschäftigung der Forstwirtschaft}

Ausgangspunkt holzbasierter Wertschöpfungsketten ist die Forstwirtschaft. Auf Basis der Forstwirtschaftlichen Gesamtrechnung (FGR) ist in Abbildung 1 die Verwendung des Bruttoproduktionswerts des Wirtschaftsbereiches Forstwirtschaft (Forstwirtschaft und Forstwirtschaftliche Dienstleistungen) in den Jahren 2006 und 2010 dargestellt. Am Bruttoproduktionswert von 3,49 Mrd. € des Jahres 2006 hatten die beschafften Vorleistungen einen Anteil von 48,5\% und der Nettounternehmensgewinn einen Anteil von 10,8 \%. Die Bruttowertschöpfung hatte einen Anteil von 51,5 \%. Mit 2,77 Mrd. € bzw. 67,45 Mio. m³ FGR-relevantem Einschlag hatte der Rohholzproduktionswert einen Anteil von 79,4 \% an der Entstehung des Bruttoproduktionswerts (grüner Balken in Abbildung 1).

Im Jahr 2010 ist der Bruttoproduktionswert des Wirtschaftsbereiches Forstwirtschaft auf 3,68 Mrd. € gestiegen. Hieran hatten die Vorleistungen einen Anteil von 50,4\% und der Nettounternehmensge- 
winn von 17,2\%. Zur Entstehung des Bruttoproduktionswerts im Jahr 2010 trug der Wert der Rohholzproduktion mit 2,87 Mrd. $€(65,48$ Mio. m³) bzw. einem Anteil von 78,0 \% bei.

Im Vergleich zum Jahr 2006 konnte der Wirtschaftsbereich Forstwirtschaft im Jahr 2010 bei einem um 2,9 \% reduzierten Einschlag demnach den Bruttoproduktionswert um 5,4 \%, die Bruttowertschöpfung um 1,4\% und den Unternehmensgewinn um 68,1\% steigern. Nach der Waldgesamtrechnung (WGR) lag die Beschäftigung 2006 bei rund 37.870 und 2010 bei rund 38.899 Vollzeitäquivalenten.

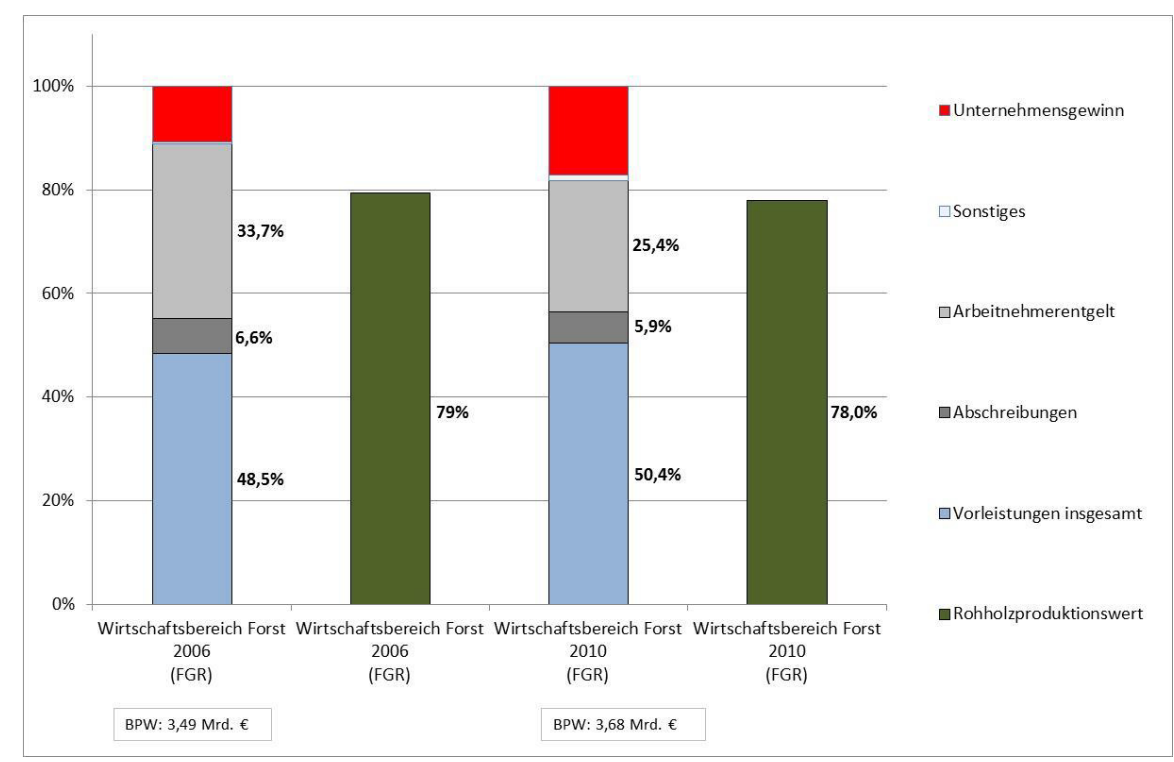

Abbildung 1: Verwendung des Bruttoproduktionswerts (BPW) des Wirtschaftsbereichs Forstwirtschaft (FGR) und Anteil des Rohholzproduktionswertes an der Entstehung

\section{Kostenstruktur und Beschäftigung der ersten Verarbeitungsstufe}

Die Verwendung des Bruttoproduktionswerts der Säge-, Hobel-, und Holzimprägnierwerke, der Hersteller von Furnier-, Sperrholz-, Holzfaser- und Holzspanplatten sowie der Hersteller von Holz- und Zellstoffindustrie ist in Abbildung 2 bis Abbildung 4 dargestellt. Bei allen drei Wirtschaftszweigen fällt auf, dass der Anteil der holzbasierten Rohstoffe, Halb- und Fertigwaren als Vorleistung im Jahr $2010 \mathrm{im}$ Vergleich zu 2006 gestiegen ist.

Die WZ 1610 Säge-, Hobel-, und Holzimprägnierwerke verzeichnete zwischen 2006 und 2010 einen Rückgang des Bruttoproduktionswerts von 4,86 Mrd. € auf 4,44 Mrd. €. Der Anteil des Bruttoproduktionsgewinns am Bruttoproduktionswert sank von 5,4\% auf 2,1\%. Die holzbasierten Vorleistungen hatten einen Anteil am Bruttoproduktionswert von 57,4 \% im Jahr 2006 und von 60,7 \% im Jahr 2010. Der Anteil von Rohholz stieg von 35,1\% in 2006 auf 41,5\% in 2010. Dagegen sank der Anteil der holzbasierten Halb- und Fertigwaren von 22,3\% auf 19,2 \% (Abbildung 2). Auch die Zahl der Beschäftigten in den Säge-, Hobel-, und Holzimprägnierwerken nahm zwischen 2006 und 2010 ab. Während im Jahr 2006 noch insgesamt 16.559 Beschäftige in den Säge-, Hobel-, und Holzimprägnierwerken arbeiteten, so waren es im Jahr 2010 nur noch 15.081 Beschäftigte (StBA: F 4. R 4.3). 
Der Bruttoproduktionswert im WZ 1621 Herstellung von Furnier-, Sperrholz-, Holzfaser- und Holzspanplatten lag im Jahr 2010 bei 5,72 Mrd. € und im Jahr 2010 bei 4,86 Mrd. €. Im Jahr 2006 hatte der Bruttoproduktionsgewinn einen Anteil am Bruttoproduktionswert von 1,4 \%. Im Jahr 2010 überstieg die Abgeltung der Vorleistungen und eigenen Produktionsfaktoren den Bruttoproduktionswert (d.h. Unternehmensverlust). Der Anteil des Bruttoproduktionsgewinns lag dementsprechend bei -2,5\% Die Hersteller von Furnier-, Sperrholz-, Holzfaser- und Holzspanplatten setzten 36,4 \% (2006) und 45,5\% (2010) des Bruttoproduktionswerts für die Beschaffung von holzbasierten Vorleistungen ein (Abbildung 3). Trotz eines Anstiegs von 7,0 \% im Jahr 2006 auf 14,0 \% im Jahr 2010 weist der WZ 1621 innerhalb der ersten Verarbeitungsstufe den geringsten Anteil von Rohholz am Bruttoproduktionswert auf. Der Einsatz von anderen holzbasierten Halb- und Fertigwaren lag dagegen im Jahr 2006 bei 29,3\% und 31,7 \% im Jahr 2010. Auch in der Holzwerkstoffindustrie sank der Anteil der Beschäftigten von $15.843 \mathrm{im} \mathrm{Jahr} 2006$ auf $12.662 \mathrm{im} \mathrm{Jahr} 2010$ (StBA: F 4. R 4.3).

Im WZ 1711 Herstellung von Holz- und Zellstoff stiegen dagegen die Beschäftigtenzahlen von 1.595 in 2006 auf 1.616 in 2010 leicht an (StBA: F 4. R 4.3). Auch der Bruttoproduktionswert stieg von 0,67 Mrd. $€$ in 2006 auf 0,88 Mrd. $€$ in 2010 (Abbildung 4). Der Anteil des Bruttoproduktionsgewinns sank jedoch in dieser Zeit von 6,9\% auf 2,9\%. Die Holz- und Zellstoffhersteller setzten im Jahr 2006 insgesamt 28,2\% des Bruttoproduktionswerts für die Anschaffung von holzbasierten Vorleistungen ein. Im Jahr 2010 lag der Anteil bei 49,1 \% (Im Jahr 2006 waren jedoch keine Ausgaben für die Warengruppe Holz- und Zellstoff in der Material und Wareneingangsstatistik ausgewiesen.). Diese deutliche Veränderung bei den holzbasierten Vorleistungen der Hersteller von Holz- und Zellstoff in der Material- und Wareneingangsstatistik könnten sich in den wenigen Erfassungseinheiten und im statistischen Datenschutz begründen. Mit 27,4\% und 27,6\% blieb der Anteil des Rohholzes am Bruttoproduktionswert der Holz- und Zellstoffhersteller in den betrachteten Jahren jedoch ungefähr gleich.

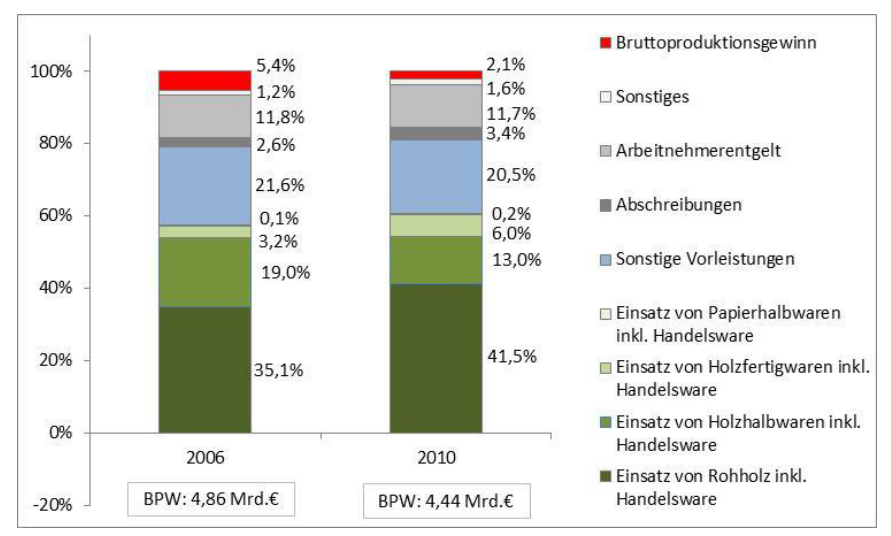

Abbildung 2: Verwendung des Bruttoproduktionswerts (BPW) in der Säge- Hobel und Holzimprägnierwerke (WZ 1610) 


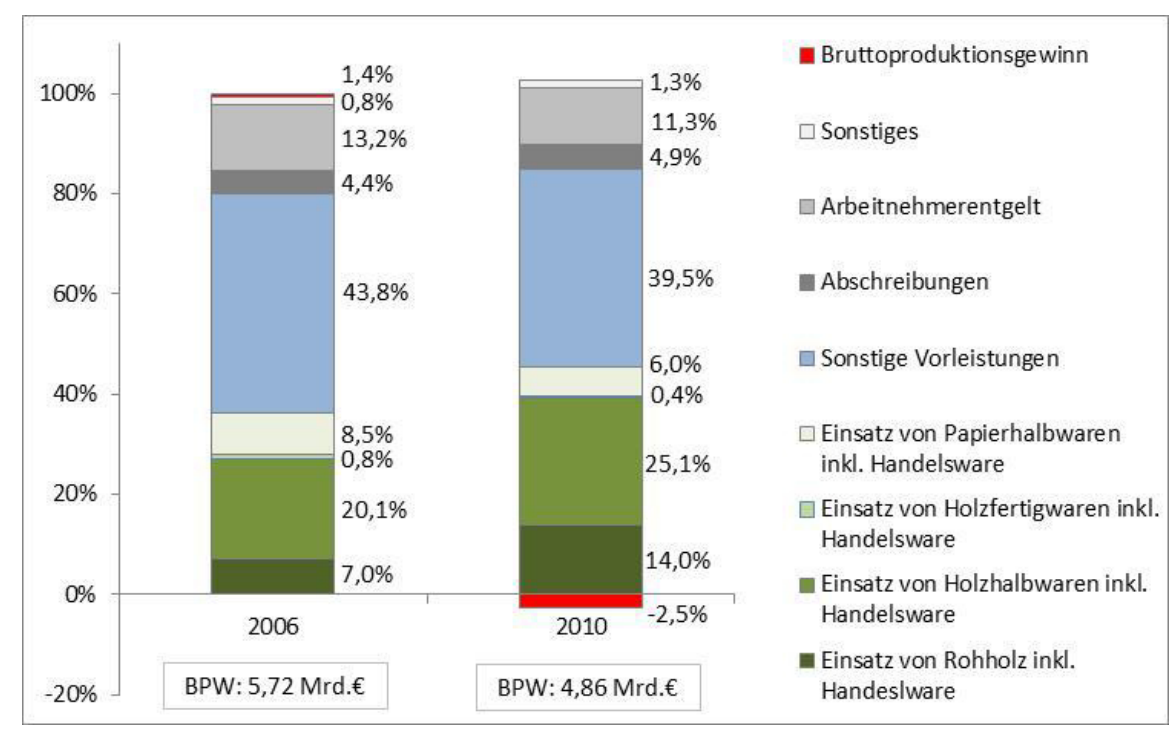

Abbildung 3: Verwendung des Bruttoproduktionswerts (BPW) der Hersteller von Furnier-, Sperrholz-, Holzfaser- und Holzspanplatten (WZ 1621)

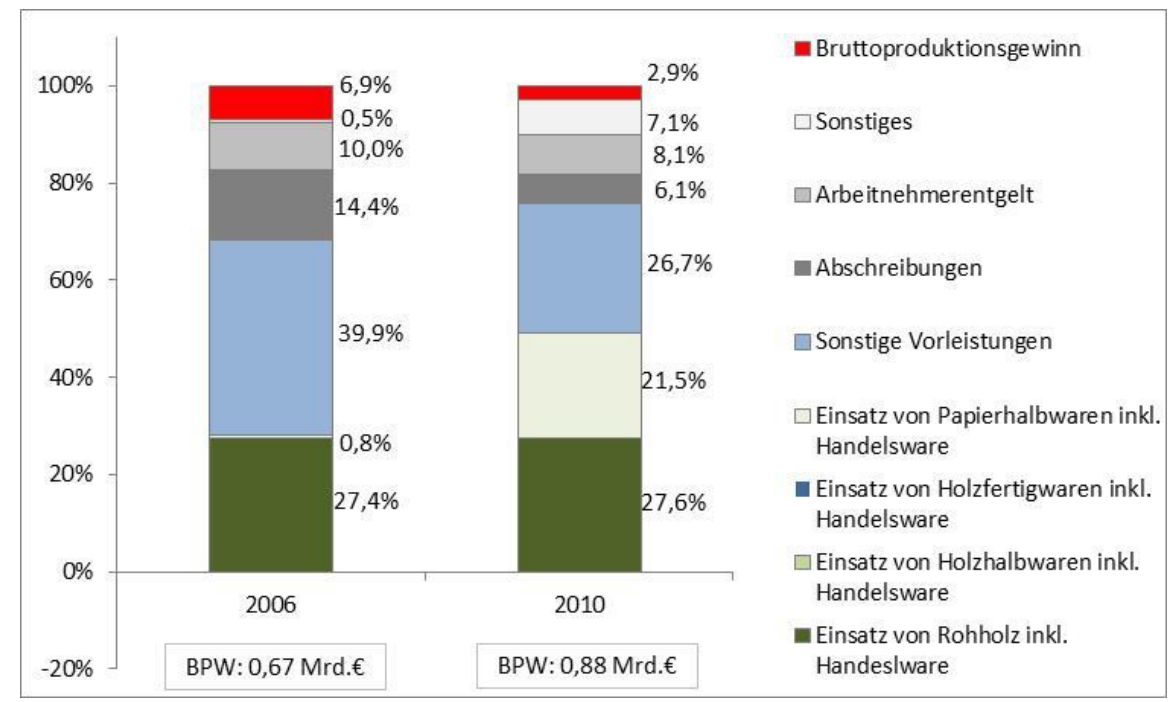

Abbildung 4: Verwendung des Bruttoproduktionswerts (BPW) der Hersteller von Holz- und Zellstoff (WZ 1711)

\section{Kostenstruktur und Beschäftigung der zweiten Verarbeitungsstufe}

Für die Darstellung der zweiten Verarbeitungsstufe wurden exemplarisch die Hersteller von sonstigen Konstruktionsteilen, Fertigbauteilen, Ausbauelementen und Fertigteilbauten aus Holz (WZ 1623), die Hersteller von Verpackungsmitteln, Lagerbehältern und Ladungsträgern aus Holz (WZ 1624), die Hersteller von Papier, Karton und Pappe (WZ 1712) und die Hersteller sonstiger Möbel (WZ 3109) ausgewählt (Abbildung 5 bis Abbildung 8). Mit Ausnahme der Hersteller sonstiger Möbel stieg der Anteil der holzbasierten Vorleistungen auch in diesen Wirtschaftszweigen in den betrachteten Jahren an. In der zweiten Verarbeitungsstufe haben die beschafften Holzhalbwaren und Papierhalbwaren den höchsten Anteil am Bruttoproduktionswert unter den holzbasierten Vorleistungen.

Im WZ 1623 Herstellung von sonstigen Konstruktionsteilen, Fertigbauteilen, Ausbauelementen und Fertigteilbauten aus Holz lag der Bruttoproduktionswert im Jahr 2006 bei 5,19 Mrd. € und stieg bis zum Jahr 2010 auf 5,31 Mrd. €. Die Unternehmen dieses Wirtschaftszweiges verzeichneten außerdem einen leichten Anstieg des Anteils des Bruttoproduktionsgewinns von 3,0 \% auf 3,5 \%. Ebenso stieg 
der Anteil holzbasierter Vorleistungen von 26,6 \% im Jahr 2006 auf 31,7 \% im Jahr 2010. Der Anteil des Einsatzes von Holzhalbwaren am Bruttoproduktionswert lag bei 24,3\% in 2006 und 28,6\% in 2010 (Abbildung 5). Die Beschäftigtenzahlen sanken um rund $8 \%$ von 36.120 in 2006 auf 33.330 in 2010 (StBA: F 4, R 4.3).

Der Bruttoproduktionswert der Hersteller von Verpackungsmitteln, Lagerbehältern und Ladungsträgern aus Holz stieg von 1,05 Mrd. € in 2006 auf 1,15 Mrd. $€$ in 2010 (Abbildung 6). Gleichzeitig stiegen auch die Beschäftigtenzahlen von 6.304 auf 6.602 an (StBA: F 4, R 4.3). Der Anteil des Bruttoproduktionsgewinns am Bruttoproduktionswert sank dagegen von 4,5\% auf 3,7\%. Die Unternehmen dieses Wirtschaftszweiges wiesen mit 45,4\% in 2006 und 47,1 \% in 2010 die höchsten Anteile von holzbasierten Vorleistungen am Bruttoproduktionswert innerhalb der zweiten Verarbeitungsstufe auf. Der Anteil der Holzhalbwaren lag bei 31,4 \% bzw. 29,2 \%.

Der WZ 1712 Herstellung von Papier, Karton und Pappe weist mit 15,51 Mrd. € im Jahr 2006 und 15,57 Mrd. $€$ im Jahr 2010 den höchsten Bruttoproduktionswert der betrachteten WZ auf. Im Jahr 2006 hatte der Bruttoproduktionsgewinn einen Anteil von 3,4 \% am Bruttoproduktionswert. Im Jahre 2010 wies die Papierindustrie hingegen einen negativen Bruttoproduktionsgewinn auf (-1,2 \%). Der Anteil holzbasierter Vorleistungen stieg von 14,8 \% im Jahr 2006 auf 17,6 \% im Jahr 2010. Der Einsatz von Papierhalbwaren hatte mit 13,1 \%, bzw. 15,5 \% den höchsten Anteil (Abbildung 7). Die Beschäftigtenzahlen sanken in der Papierindustrie zwischen 2006 und 2010 um rund 11\%. Während im Jahr 2006 noch 40.669 Personen beschäftigt waren, betrug die Beschäftigung in 2010 nur noch 36.326 Personen (StBA: F 4, R 4.3).

Der Bruttoproduktionswert der Hersteller von sonstigen Möbeln (WZ 3109) stieg von 6,4 Mrd. € im Jahr 2006 auf 8,33 Mrd. € in 2010 (Abbildung 8). Auch die Beschäftigtenzahlen stiegen in dieser Zeit von 43.625 auf 55.010 Personen. Die Ursache für diesen deutlichen Anstieg des Bruttoproduktionswerts und der Beschäftigung dürfte überwiegend auf die Umstellung von WZ 2003 auf WZ 2008 zurückzuführen sein. In der WZ 2008 wurden den Herstellern sonstiger Möbel die ehemaligen Hersteller von Schlafzimmer-, Wohn- und Esszimmermöbel der WZ 2003 zugeordnet. Der Anteil des Bruttoproduktionsgewinns am Bruttoproduktionswert stieg von 4,2\% auf 4,4\% an. Der Anteil holzbasierter Vorleistungen betrug 22,8 \% im Jahr 2006 und 16,9 \% im Jahr 2010. Die Holzhalbwaren hatten 2006 einen Anteil von 13,9\% und 2010 einen Anteil von 11,5\% am Bruttoproduktionswert. 


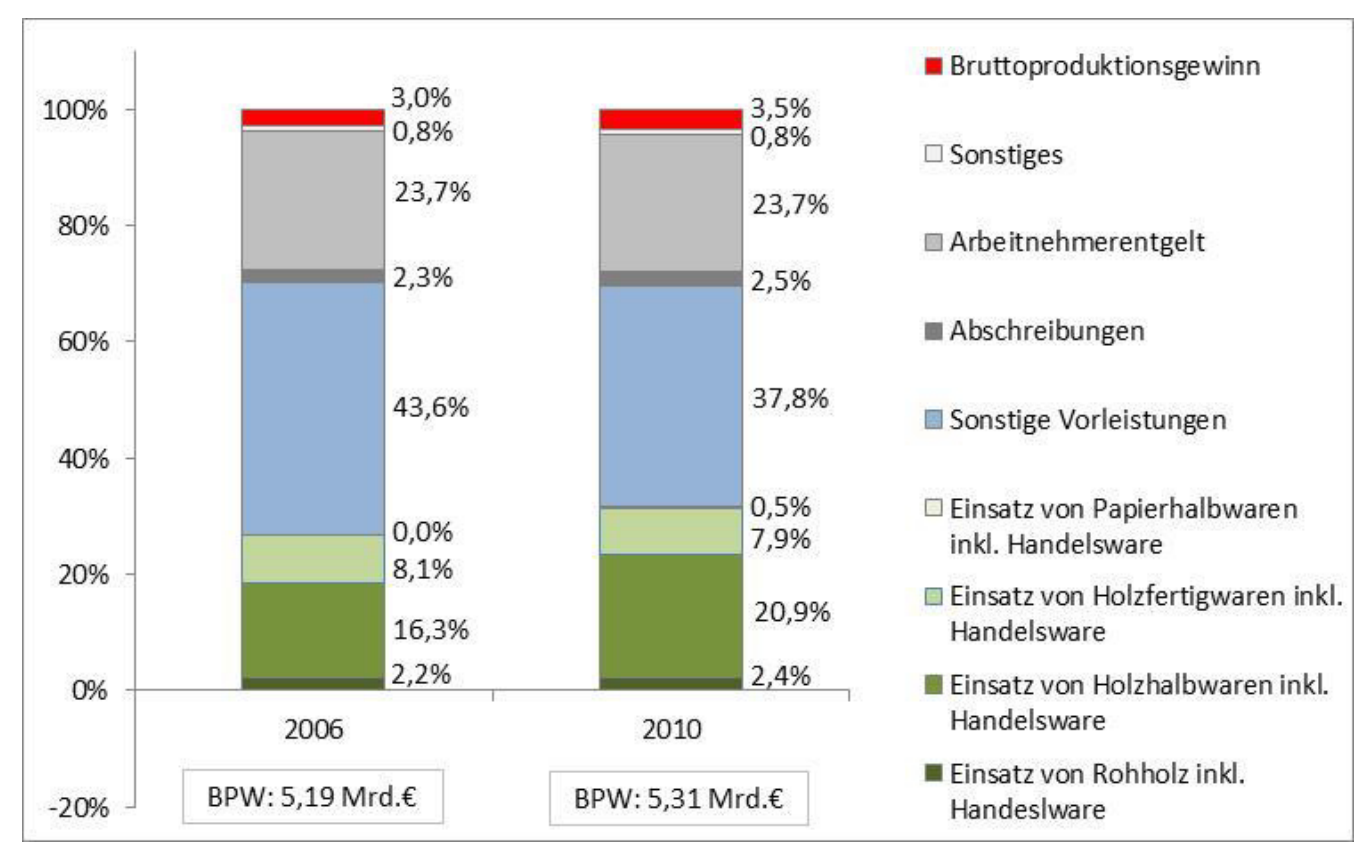

Abbildung 5: Verwendung des Bruttoproduktionswerts (BPW) bei den Herstellern von Konstruktionsund Fertigbauteilen aus Holz (WZ 1623)

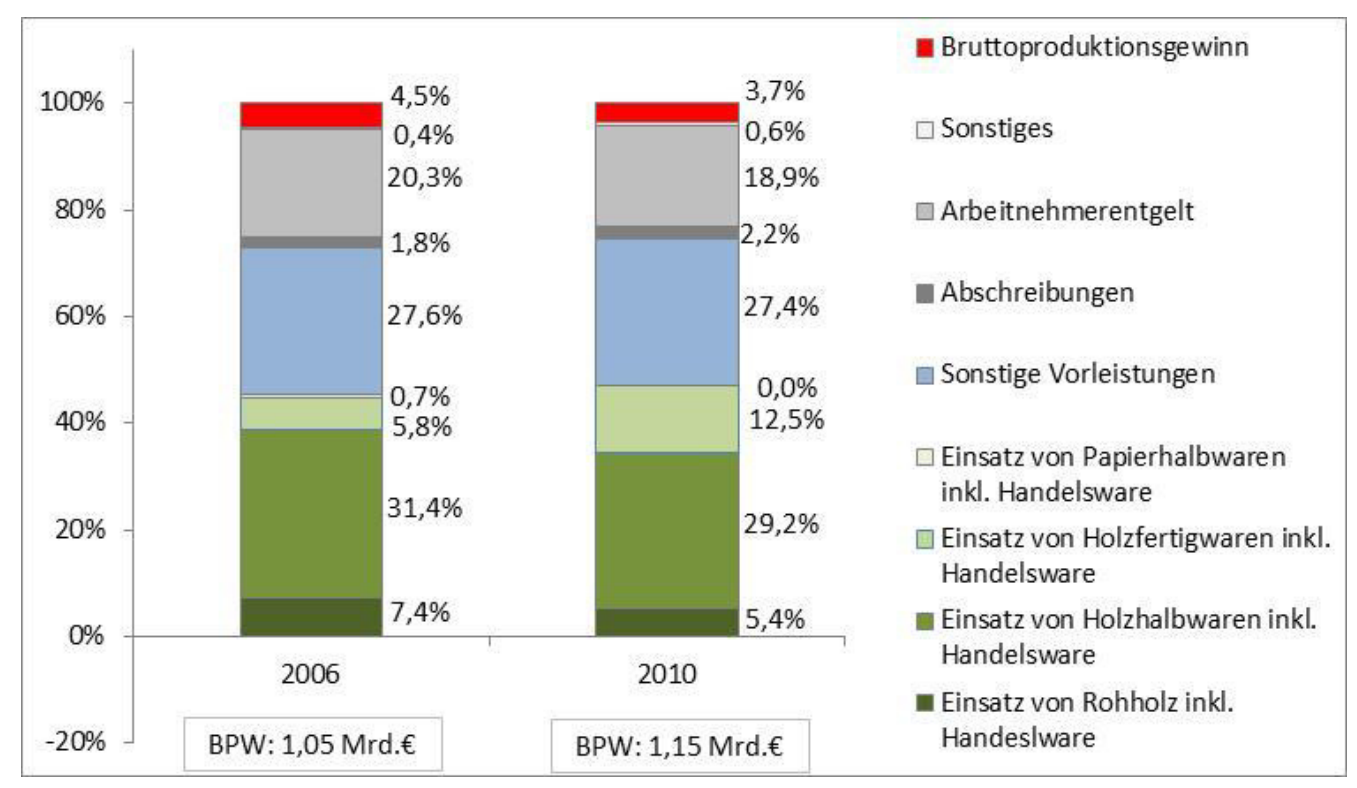

Abbildung 6: Verwendung des Bruttoproduktionswerts (BPW) in der Holzpackmittelindustrie (WZ 1624) 


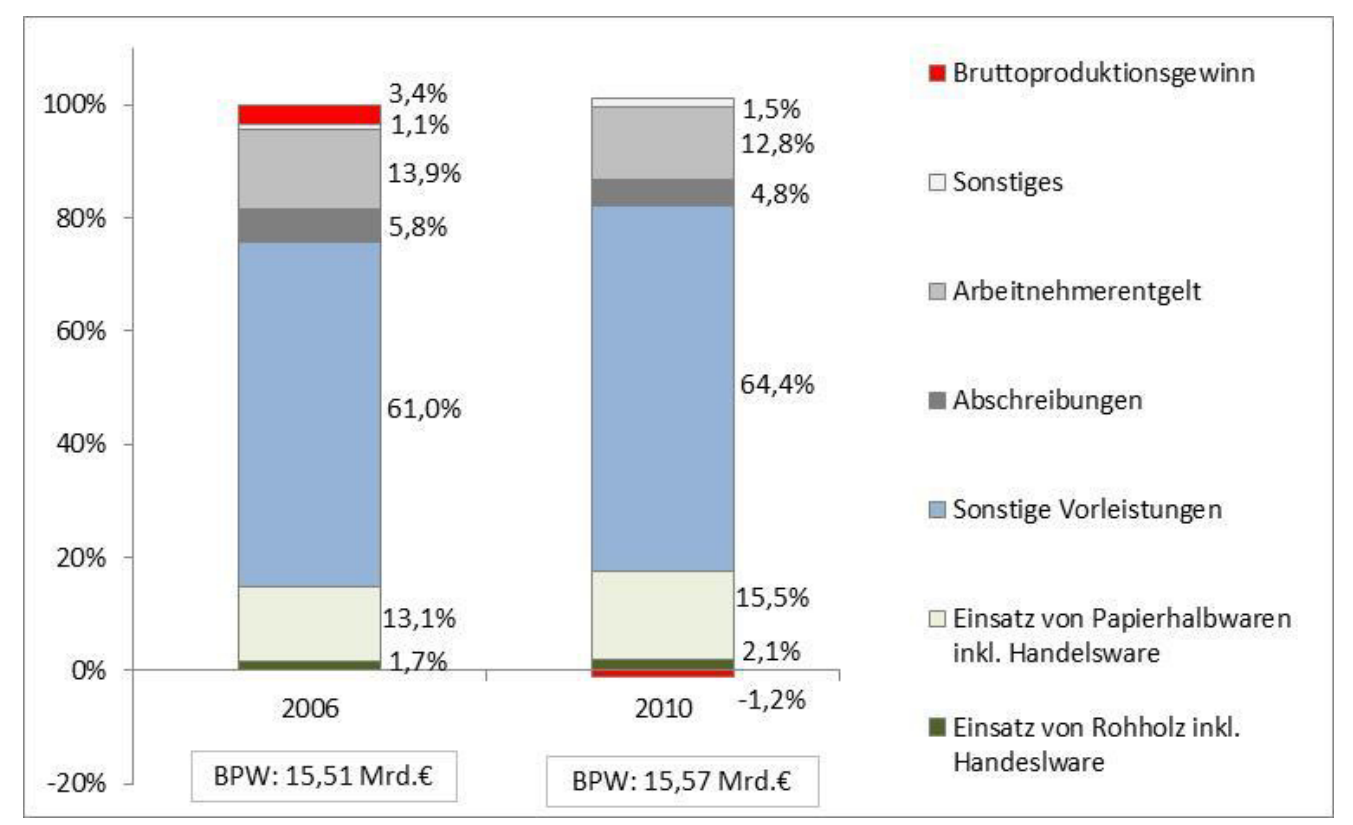

Abbildung 7: Verwendung des Bruttoproduktionswerts (BPW) in der Papierindustrie (WZ 1712)

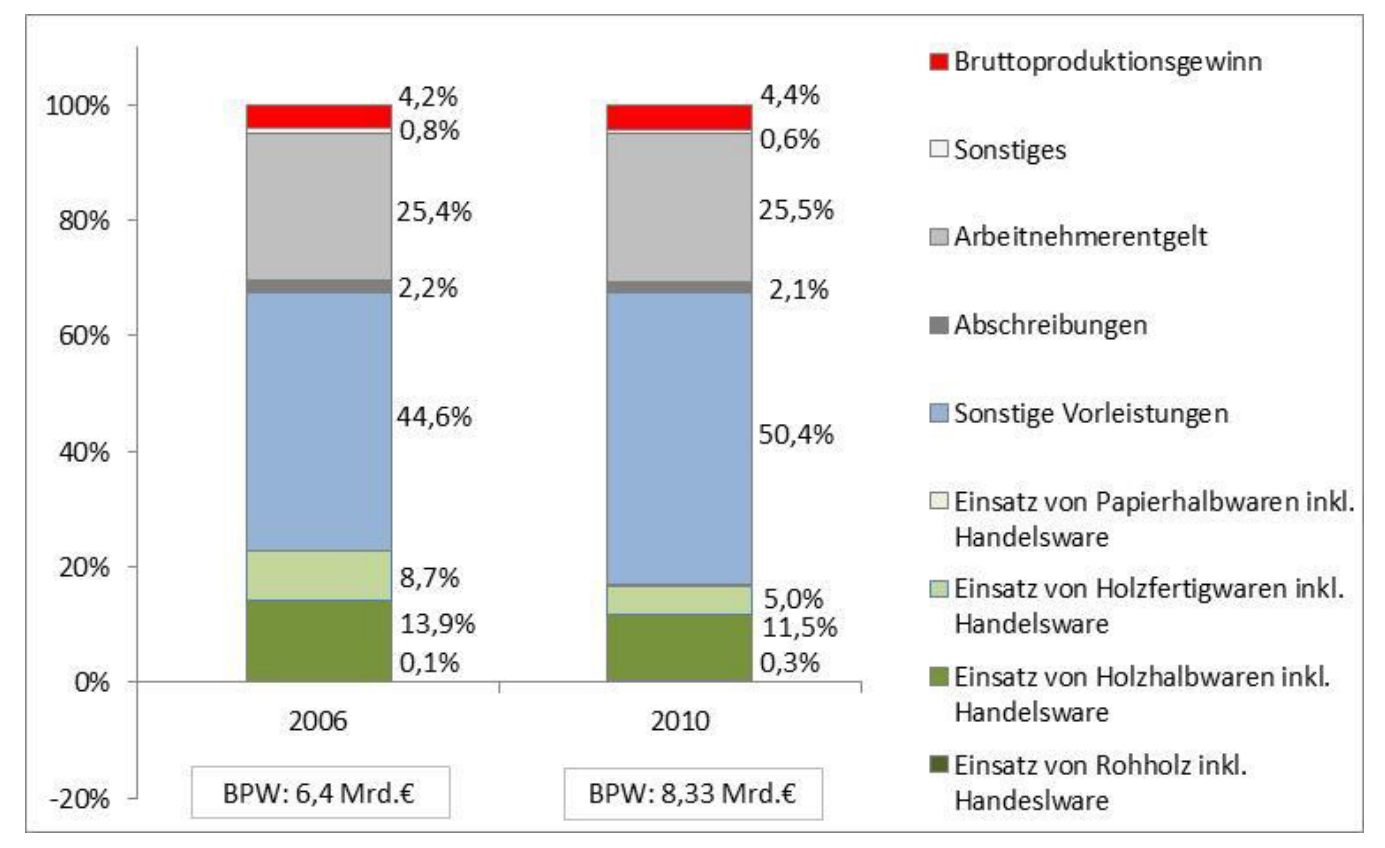

Abbildung 8: Verwendung des Bruttoproduktionswerts (BPW) bei den Herstellern von sonstigen Möbeln (WZ 3109)

\section{Abschätzung der holzbasierten Wertschöpfungsketten und Beschäftigung}

Auf Grundlage der vorgestellten Daten soll nachfolgend ein Ansatz zur Abschätzung der holzbasierten Wertschöpfung und Beschäftigung für ausgewählte Wertschöpfungsketten vorgestellt werden. Hierbei wurde berechnet, welche Wertschöpfung und Beschäftigung im Jahr 2010 durch den Einsatz einer durchschnittlichen Einheit holzbasierter Rohstoffe, Halb- und Fertigwaren im jeweiligen Wirtschaftszweige entsteht. Mit den isoliert für die Wirtschaftszweige berechneten Kennzahlen wurden idealtypische Wertschöpfungsketten konstruiert und die holzbasierte Wertschöpfung und Beschäftigung der einzelnen Wertschöpfungskettenglieder im Anschluss addiert. Den tatsächlichen „Holzfaserfluss“, wie 
von Weimar (2011) dargestellt, bildet diese Abschätzung somit nicht ab. Dieser Ansatz wurde gewählt, da in der Wirtschaftspraxis die einzelnen Wirtschaftszweige durch Beschaffung und Absatz von holzbasierten Rohstoffen, Halb- und Fertigwaren auf unterschiedlichsten Ebenen vernetzt sind und eine idealtypisch, linear-funktionale Verbindung zwischen den Wirtschaftszweigen nicht herstellbar ist.

Während durch die Forstwirtschaftliche Gesamtrechnung (FGR) Werte und Menge des produzierten Rohholzes vorliegen und dieses direkt in Bezug zur Wertschöpfung und Beschäftigung des Wirtschaftsbereiches Forstwirtschaft gesetzt werden kann, liegen durch die Material- und Wareneingangsstatistik ausschließlich absolute Warenwertangaben zu Anschaffungskosten der einzelnen Warengruppen vor. Vor diesem Hintergrund wurde unterstellt, dass der Wareneingang der Wirtschaftszweige in der Material- und Wareneingangsstatistik in seiner wert- und mengenmäßigen Struktur der tiefer differenzierten Produktionsstatistik des Verarbeitenden Gewerbes (StBA F4 R3.1) entspricht. Während die Kostenstrukturstatistik sowie die Material- und Wareneingangserhebung Unternehmen nach dem Schwerpunktprinzip klassifiziert, beinhaltet die Produktionserhebung die Produktion des gesamten Verarbeitenden Gewerbes. Die Art der abgefragten Daten und die Zuordnung weichen demnach voneinander ab.

Um den Wert einer Einheit holzbasierter Rohstoffe, Halb- und Fertigwaren über mehrere Warengruppen der Material- und Wareneingangsstatistik im Durchschnitt zu berechnen, wurden die ausgewiesenen Einheiten $\left(\mathrm{m}^{3}, \mathrm{~m}^{2}, \mathrm{~m}\right.$, Stück, Tonne) der Produktionserhebung zunächst in die Einheit Kubikmeter umgerechnet. Da in der Material- und Wareneingangsstatistik die Warengruppen zu Anschaffungskosten bewertet sind und in der Produktionserhebung die produzierten Einheiten als Verkaufspreise ab Werk, wurden pauschal $10 € / \mathrm{m}^{3}$ Transportkosten zur Ermittlung der Anschaffungskosten aufgeschlagen. Hierauf aufbauend wurde mengen- und preisgewichtet der durchschnittliche Wert einer Einheit holzbasierter Rohstoffe, Halb- und Fertigwaren der Wirtschaftszweige in der Material- und Wareneingangsstatistik bestimmt. Auf Basis dieser abgeschätzten Preise wurden die holzbasierten Vorleistungen der ersten und zweiten Verarbeitungsstufe in Beziehung zur Bruttowertschöpfung und Beschäftigung gesetzt.

Auf Basis dieser Berechnungen ist in Tabelle 2 die holzbasierte Wertschöpfung und Beschäftigung für die exemplarisch ausgewählten Wertschöpfungsketten „Holzhaus“, „Sonstige Möbel“, „Verpackung“ und „Papier“ pro Einheit produziertem Rohholz bzw. holzbasierter Vorleistungen ausgewiesen. Zu beachten ist hierbei, dass aufgrund des gewählten Ansatzes in den Wertschöpfungsketten nicht sämtliche holzbasierten Vorleistungen der einzelnen Wirtschaftszweige von den vorgelagerten Wertschöpfungskettengliedern bezogen werden.

Tabelle 2: Holzbasierte Bruttowertschöpfung und Beschäftigung in ausgewählten Wertschöpfungsketten (eigene Berechnungen für das Jahr 2010)

\begin{tabular}{|l|c|c|}
\hline Wertschöpfungskette & $\begin{array}{c}\text { Bruttowertschöpfung je } \\
\text { 1 Einheit produzierten Roh- } \\
\text { holzes bzw. holzbasierter } \\
\text { Vorleistungen }\end{array}$ & $\begin{array}{c}\text { Beschäftigte je 1.000 Einheiten } \\
\text { produzierten Rohholzes bzw. } \\
\text { holzbasierter Vorleistungen }\end{array}$ \\
\hline
\end{tabular}




\begin{tabular}{|c|c|c|}
\hline \multicolumn{3}{|l|}{ "Holzhaus" } \\
\hline Forstwirtschaft & $29 €$ & 0,5 \\
\hline WZ 1610 Sägeindustrie & $35 €$ & 0,7 \\
\hline WZ 1623 H.v. sonst. Konstr.-, Fertigbauteilen u.Ä. aus Holz & $208 €$ & 4,3 \\
\hline Summe für Wertschöpfungskette "Holzhaus" & $273 €$ & 5,5 \\
\hline \multicolumn{3}{|l|}{ "Sonstige Möbel" } \\
\hline Forstwirtschaft & $29 €$ & 0,5 \\
\hline WZ 1621 Holzwerkstoffindustrie & $61 €$ & 1,1 \\
\hline WZ 3109 H. v. sonstigen Möbeln & $598 €$ & 12,1 \\
\hline Summe für Wertschöpfungskette "Sonstige Möbel" & $688 €$ & 13,7 \\
\hline \multicolumn{3}{|l|}{ "Verpackung" } \\
\hline Forstwirtschaft & $29 €$ & 0,5 \\
\hline WZ 1610 Sägeindustrie & $35 €$ & 0,7 \\
\hline WZ 1624 H.v. Verpackungsmitteln, Lagerbehältern u.Ä. a. Holz & $82 €$ & 1,8 \\
\hline Summe für Wertschöpfungskette "Verpackung" & $146 €$ & $3, \mathbf{0}$ \\
\hline \multicolumn{3}{|l|}{ "Papier" } \\
\hline Forstwirtschaft & $29 €$ & 0,5 \\
\hline WZ 1711 Holz- und Zellstoffhersteller & $59 €$ & 0,4 \\
\hline WZ 1712 H. v. Papier, Karton und Pappe & $2.559 €$ & 33,2 \\
\hline Summe für Wertschöpfungskette "Papier" & $2.647 €$ & $\mathbf{3 4 , 1}$ \\
\hline
\end{tabular}

Aus Tabelle 3 wird deutlich, dass in der Wertschöpfungskette „Papier“ im Jahr 2010 die höchste Bruttowertschöpfung $\left(2.647 € / \mathrm{m}^{3}\right)$ und Beschäftigung $\left(34,1\right.$ Beschäftigte/1.000 $\left.\mathrm{m}^{3}\right)$ generierte wurde. An zweiter Stelle folgt die Herstellung sonstiger Möbel $\left(688 € / \mathrm{m}^{3}, 13,7\right.$ Beschäftigte $\left./ 1.000 \mathrm{~m}^{3}\right)$, an dritter Stelle der „Holzhausbau“ (273 €/m³, 5,5 Beschäftigte $/ 1.000 \mathrm{~m}^{3}$ ) und an letzter Stelle die Herstellung

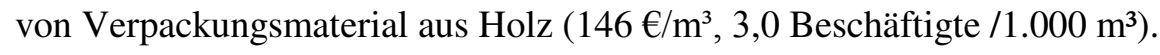

\section{Zusammenfassung und Schlussfolgerungen}

Mit diesem Beitrag wurde die Stellung der holzbasierten Vorleistungen in der Kostenstruktur ausgewählter Wirtschaftszweige in den Jahren 2006 und 2010 aufgezeigt. Mit Ausnahme der Hersteller sonstiger Möbel stiegen in allen Wirtschaftszweigen der ersten und zweiten Verarbeitungsstufe die Anteile der Ausgaben für Rohholz und sonstige holzbasierte Vorleistungen am Bruttoproduktionswert zwischen 2006 und 2010. In der ersten Holzverarbeitungsstufe haben holzbasierte Vorleistungen, mit $28 \%$ bis $58 \%$ in 2006 und $45 \%$ bis $61 \%$ in 2010, einen hohen Anteil am Bruttoproduktionswert. Mit bis zu 41,5\% ist der Anteil von Rohholz als Vorleistung am Bruttoproduktionswert in der Sägeindustrie am höchsten. In der zweiten Verarbeitungsstufe lag der Anteil von holzbasierten Vorleistungen am Bruttoproduktionswert in 2006 zwischen $15 \%$ und $45 \%$ und in 2010 zwischen $17 \%$ und $47 \%$.

In fünf Wirtschaftszweige sank der Bruttoproduktionsgewinn zwischen den Jahren 2006 und 2010. In der Holzwerkstoffindustrie und der Papierindustrie überstieg die Abgeltung der Vorleistungen und eigenen Produktionsfaktoren im Jahr 2010 den Bruttoproduktionswert, so dass ein Unternehmensverlust verzeichnet wurde. Bei weiter steigenden Kosten für holzbasierte Vorleistungen könnte sich die wirtschaftliche Situation dieser Unternehmen verschärfen.

Aufbauend auf den Analysen zur Kostenstruktur der holzverarbeitenden Wirtschaftszweige wurde ein Ansatz zur Abschätzung der holzbasierten Wertschöpfung und Beschäftigung für unterschiedliche Wertschöpfungsketten vorgestellt. Mit diesen Abschätzungen wurde aufgezeigt, welche Wertschöp- 
fung und Beschäftigung durch den Einsatz einer durchschnittlichen Einheit holzbasierter Vorleistungen in den einzelnen Wirtschaftszweigen generiert wird. Um für jeden Wirtschaftszweig den Einsatz einer durchschnittlichen Einheit holzbasierter Rohstoffe, Halb- und Fertigwaren berechnen zu können, wurden vereinfacht die wert- und mengenmäßige Struktur der Produktionsstatistik aufgrund fehlender Datenquellen unterstellt. Für die Wertschöpfungsketten „Holzhaus“, „Sonstige Möbel“, „Verpackung“ und „Papier“ lässt sich mit dieser Abschätzung eine Wertschöpfung zwischen $146 €$ und $2647 €$ bezogen auf eine Einheit produzierten Rohholzes bzw. holzbasierter Vorleistungen, und eine Beschäftigung von 5,5 bis 34,1 Personen pro 1.000 Einheiten produzierten Rohholzes bzw. holzbasierter Vorleistungen, ausweisen.

Von Schweinle (2012) wurde die Bruttowertschöpfung bei der energetischen Holzverwendung (Waldhackschnitzelverwendung sowie Scheitholzverwendung im Hausbrand, jeweils einschließlich Holzernte) in einer Größenordnung von $70 € / \mathrm{m}^{3}$ berechnet. Die Bruttowertschöpfung der energetischen Holzverwendung bewegt sich demnach in einer vergleichbaren Größenordnung wie die Bruttowertschöpfung der stofflichen Holzverwendung bis zur Halbwarenebene (d.h. Forstwirtschaft und erste Verarbeitungsstufe: 64 bis $90 € / \mathrm{m}^{3}$ (Tabelle 2)). In den stofflichen Wertschöpfungsketten werden die größeren Wertschöpfungsanteile jedoch in der zweiten Verarbeitungsstufe generiert.

\section{Literatur}

BECHER, G. (2014): Clusterstatistik Forst und Holz: Tabellen für das Bundesgebiet und die Länder 2000 bis 2012 . Thünen Working Paper 32, Hamburg

BUNDESMINISTERIUM FÜR ERNÄHRUNG UND LANDWIRTSCHAFT (BMEL) (2014): Der

Wald in Deutschland: Ausgewählte Ergebnisse der dritten Bundeswaldinventur. Berlin

DÖRING, P. und MANTAU, U. (2012): Standorte der Holzwirtschaft: Sägeindustrie: Einschnitt und Sägenebenprodukte 2010. Universität Hamburg, Zentrum für Holzwirtschaft. Hamburg

MANTAU, U. (2012): Holzrohstoffbilanz Deutschland: Entwicklungen und Szenarien des Holzaufkommens und der Holzverwendung 1987 bis 2015, Hamburg 65 S.

SCHWEINLE, J. (2012): Wertschöpfungsanalyse der energetischen Nutzung von Holz, in: Arbeitsbericht, Nr. 2012/02, vTI - Institut für Ökonomie der Forst- und Holzwirtschaft (Hrsg.), Hamburg SEINTSCH, B. (2011): Stellung der Holzrohstoffe in der Kostenstruktur des Holz- und Papiergewerbes in Deutschland. Arbeitsbericht des Instituts für Ökonomie der Forst- und Holzwirtschaft 03/2011, Hamburg

SEINTSCH, B. (2013): Cluster Forst und Holz nach neuer Wirtschaftszweigklassifikation: Tabellen für das Bundesgebiet und die Länder 2000 bis 2011. Thünen Working Paper 5, Hamburg STATISTISCHES BUNDESAMT (StBA): Material- und Wareneingangserhebung im Verarbeitenden Gewerbe sowie im Bergbau und in der Gewinnung von Steinen und Erden (F4 R4.2.4), Wiesbaden (jährlich) 
STATISTISCHES BUNDESAMT (StBA): Kostenstruktur der Unternehmen des Verarbeitenden Gewerbes sowie des Bergbaus und der Gewinnung von Steinen und Erden (F4 R4.3), Wiesbaden (jährlich)

STATISTISCHES BUNDESAMT (StBA): Produktionserhebungen (F4 R3.1) Wiesbaden (jährlich) STATISTISCHES BUNDESAMT (StBA): Erzeugerpreisindizes der Produkte des Holzeinschlags aus den Staatsforsten. Statistik 61231, Genesis-Online Datenbank

WEIMAR, H. (2011): Der Holzfluss in der Bundesrepublik Deutschland 2009: Methode und Ergebnis der Modellierung des Stoffflusses von Holz. Arbeitsbericht des Instituts für Ökonomie der Forst- und Holzwirtschaft 2011/06, Hamburg 
4. haverkamp M, Henke S, Kleinschmit C, Möhring B, Müller H, Mubhoff O, Rosenkranz L, Seintsch B, SCHLOSSER K, und THEUVSEN L (2014): Vergleichende Bewertung der Nutzung von Biomasse: Ergebnisse aus den Bioenergieregionen Göttingen und BERTA. Diskussionspapier Department Agrarökonomie und Rurale Entwicklung, Universität Göttingen Nr. 1405:22-26 


\section{Diskussionspapiere}

\section{Discussion Papers}

Göttingen, Mai 2014

Vergleichende Bewertung der Nutzung von Biomasse:

Ergebnisse aus den Bioenergieregionen Göttingen und BERTA

M. Haverkamp, S. Henke, C. Kleinschmit, B. Möhring, H. Müller,

O. Mußhoff, L. Rosenkranz, B. Seintsch, K. Schlosser und L. Theuvsen

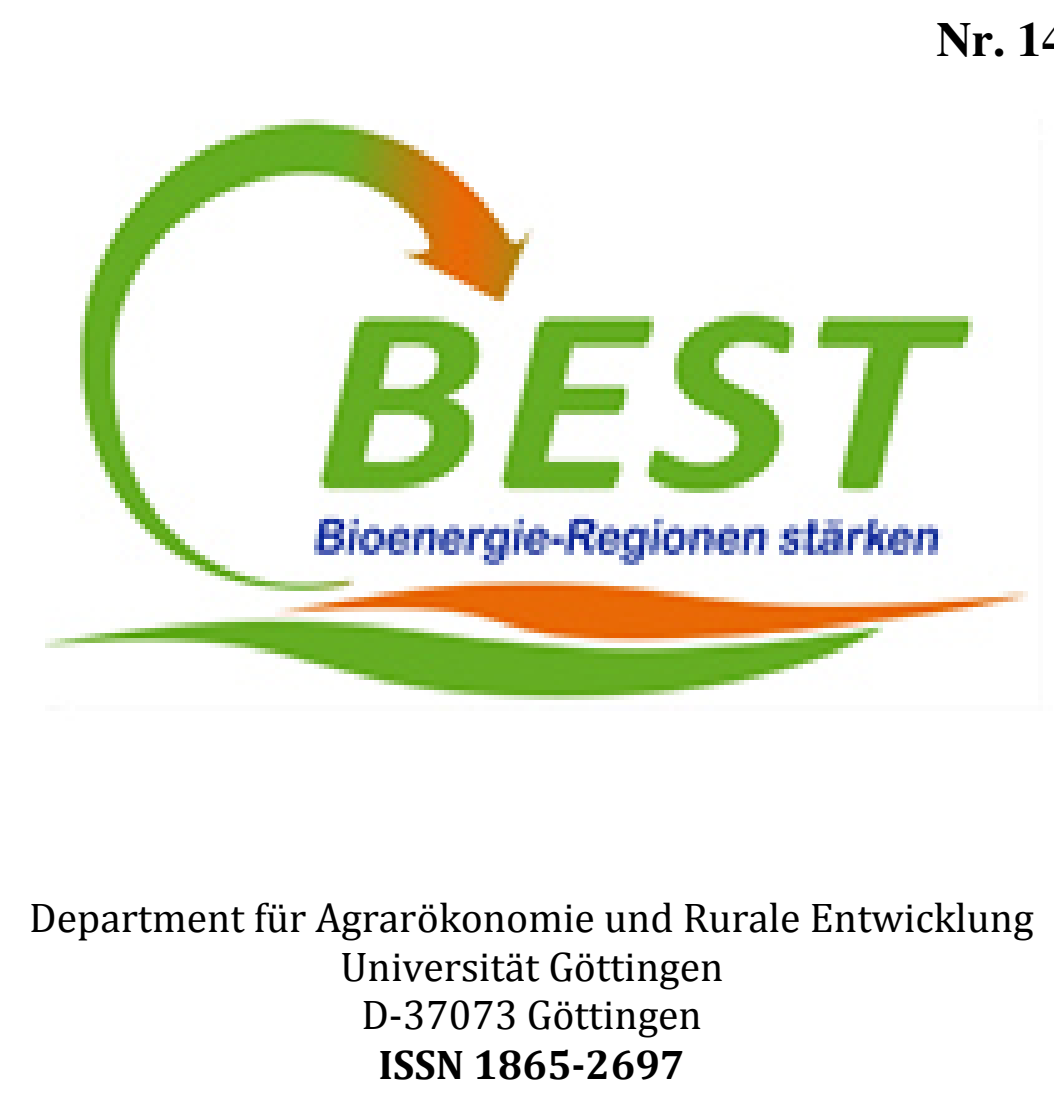




\section{Inhalt}

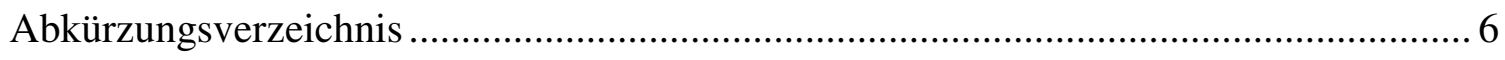

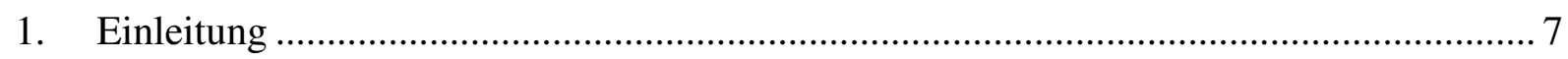

2. Beschreibung der Untersuchungsregionen ....................................................................

2.1 Agrarstrukturen in den Untersuchungsregionen ................................................. 8

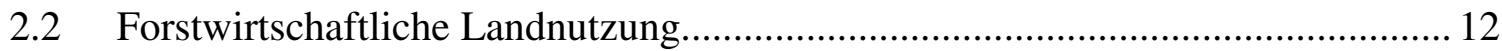

3. Teilprojekte des Clusters SÖB ……………………………………………………. 15

3.1 Ökonomische Analyse der Energieholzbereitstellung............................................ 15

Problemstellung und Zielsetzung ................................................................................ 15

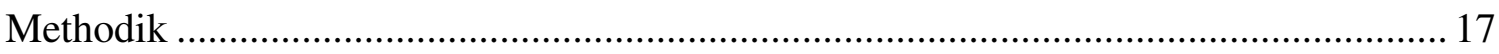

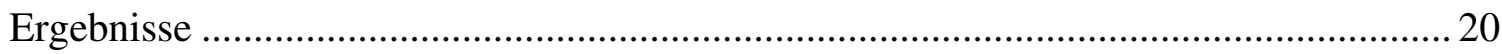

3.2 Wertschöpfungsanalyse von Waldflächen und Rohholz.........................................2 22

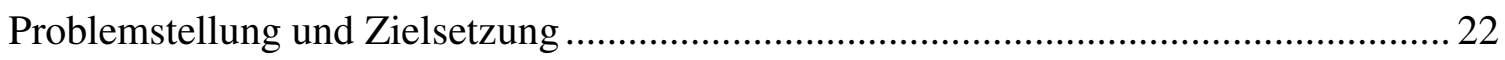

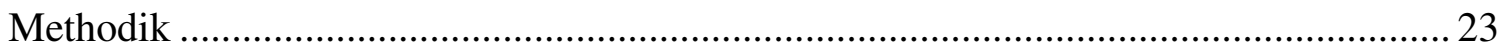

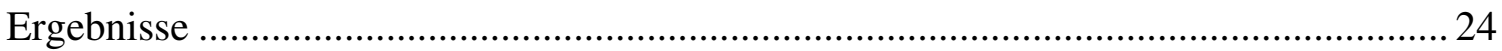

Bezug zu den Untersuchungsregionen ........................................................................... 25

3.3 Miscanthus und Pappelplantagen im Kurzumtrieb als Alternative zum klassischen Ackerbau ……………………………………………………... 28

3.4 Social Life Cycle Assessment von Biogas und Kurzumtriebsplantagen ................ 31

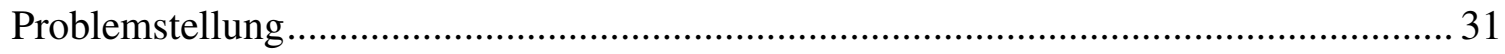

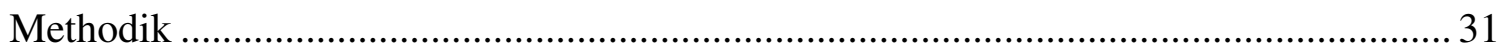

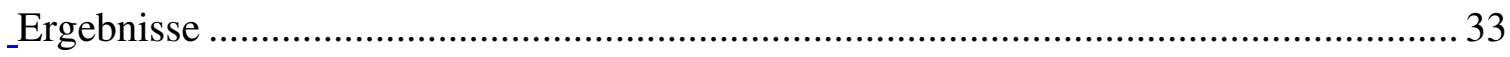

4. Schlussfolgerungen für die Untersuchungsregionen ........................................................ 36

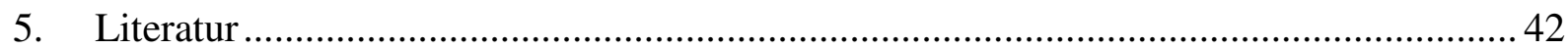




\section{Abkürzungsverzeichnis}

a Jahr

Abb. Abbildung

AK-E Arbeitskräfteeinheit

FGR Forstliche Gesamtrechnung

Fm Festmeter

ha Hektar

KUP Kurzumtriebsplantage

LF landwirtschaftlich genutzte Fläche

LK Landkreis

LKD Leistungs-Kostendifferenz

RBS Randomized Branch Sampling Verfahren

SETAC Society of Environmental Toxicology and Chemistry

SLCA Social Life Cycle Assessment

SÖB Sozioökonomische Bewertung

Tab. Tabelle

WK Wertschöpfungskette 
tigung finden müssen. Die Integration der Ergebnisse der Zeitverbrauchsstudie in den Optimierungsalgorithmus steht allerdings noch aus.

\subsection{Wertschöpfungsanalyse von Waldflächen und Rohholz}

\section{Problemstellung und Zielsetzung}

In Folge steigender, teilweise konkurrierender gesellschaftlicher Anforderungen nehmen die Nutzungskonflikte um Waldflächen zu. Beispielsweise werden im Rahmen wirtschafts- und energiepolitischer Ziele die Steigerung der energetischen und stofflichen Holznutzung angestrebt (Charta für Holz, Biomasseaktionsplan) und gleichzeitig zur Verwirklichung aktueller naturschutzpolitischer Ziele Nutzungsextensivierungen und Stilllegungsflächen (FFH-Richtlinie, $5 \%$-Ziel der Biodiversitätsstrategie) gefordert. Implementierungsebene von konkreten Umsetzungsstrategien, die Auswirkungen auf die waldflächen- und holzbasierte Wertschöpfung haben, sind Regionen. Wichtige Kennzahlen für die Bewertung unterschiedlicher Nutzungsoptionen sind die Wertschöpfung und die Beschäftigung. „Die Bruttowertschöpfung umfasst - nach Abzug sämtlicher Vorleistungen - die insgesamt produzierten Güter und Dienstleistungen zu den am Markt erzielten Preisen und ist somit der Wert, der den Vorleistungen durch Bearbeitung hinzugefügt worden ist" (StBA: F 4 R 4.3:11). Die Bruttowertschöpfung zu Faktorkosten wird durch Subtraktion sonstiger indirekter Steuern und durch Addition von Subventionen von der Bruttowertschöpfung berechnet (StBA: F 4 R 4.3).

Für eine erste Bewertung konkurrierender waldflächen- und rohholzbasierter Nutzungsoptionen dürfte die Datengrundlage (z.B.: Waldfläche, Altersklassenverteilung, Baumartenanteile, Einschlag) in Regionen unzureichend oder aufwendig zu erheben sein. Belastbare Wertschöpfungsanalysen auf regionaler Ebene sind zudem bislang mit hohem Aufwand verbunden. Daher soll im Rahmen der Teilstudie SÖB 2 ein einfaches Verfahren zur Abschätzung von Wertschöpfung und Beschäftigung unterschiedlicher Rohholznutzungsoptionen in Regionen vorgestellt werden. Auf Basis orientierender Größenordnungen für die Bewertung von Nutzungsoptionen bzw. Opportunitätskosten der Waldflächen- und Rohholznutzung und Kenntnissen der regionalen Wertschöpfungskettenglieder (bzw. Vorhandensein von Sägewerken, Biomassehöfen,...) können mit diesem Verfahren einfache regionalspezifische Abschätzungen von Wertschöpfung und Beschäftigung durchgeführt werden. 


\section{Methodik}

Um Kennzahlen zur Wertschöpfung und Beschäftigung pro Hektar Waldflächen sowie pro Einheit Rohholz abzuschätzen, wurden zunächst die Bruttowertschöpfung und Beschäftigung für den Wirtschaftsbereich Forstwirtschaft, welcher die Teilbereiche Forstwirtschaft (ohne Erbringung forstwirtschaftlicher Dienstleistungen) und forstliche Dienstleister umfasst, aus der Forstlichen Gesamtrechnung (FGR) abgeleitet. Die FGR wird jährlich erstellt und bietet bundesweite Durchschnittswerte zur Entstehung der Güter und des Einkommens im Wirtschaftsbereich Forstwirtschaft. Das methodische Vorgehen ist bei DIETER et al. (2004) ausführlich beschrieben. Zur Analyse der Wertschöpfungs- und Beschäftigungseffekte des Wirtschaftsbereiches Forstwirtschaft werden die FGR-Ergebnisse des Jahres 2006 verwendet (DIETER \& KÜPPERS, 2008).

Zur Ermittlung ökonomischer Kennzahlen der Wertschöpfung durch energetische Holznutzung wurden von SCHWEINLE (2012) Wertschöpfungskettenanalysen, basierend auf Investitionsrechnungen einzelner Kettenglieder, bis zum Endverbraucher durchgeführt. Zur Ermittlung der Wertschöpfung durch stoffliche Holznutzung bis zur ersten Verarbeitungsstufe (z. B. Erzeugung von Halbwaren) wurden für einzelne Glieder der holzbasierten Wertschöpfungsketten auf Basis der Kostenstrukturstatistik (StBA: Fachserie 4, Reihe 4.3) sowie der Material- und Wareneingangserhebung (StBA: Fachserie 4, Reihe 4.2.4) die Bruttowertschöpfung und Beschäftigung für sortimentsspezifische Holzrohstoffkosten ermittelt (SEINTSCH. 2011). Die durchschnittlichen Beschäftigungszahlen der stofflichen Holznutzung wurden für die untersuchten Sektoren aus der Kostenstrukturstatistik entnommen. Zusätzlich wurden für die energetische Wertschöpfungsketten durchschnittliche Beschäftigungszahlen mittels des Jahresdurchschnittsverdienstes eines Arbeitnehmers im Forstbetrieb oder bei Forstlichen Dienstleistern von $31.214 € /$ a (FGR 2006) abgeleitet. In Verbindung mit der Arbeit von SCHWEINLE (2012) wurde so ein Vergleich zwischen stofflichen und energetischen Wertschöpfungsketten ermöglicht. Es ist jedoch zu berücksichtigen, dass die Analyse der stofflichen Wertschöpfungskette - im Gegensatz zu energetischen Wertschöpfungskette - bisher nur bis zur Halbwarenebene erfolgt ist und sich andere Größenordnungen für vollständige Wertschöpfungsketten bis zur Weiterverarbeitung zu Fertigwaren ergeben werden.

In einem weiteren Arbeitsschritt wurden die Ergebnisse auf die Regionen Göttinger Land und BERTA übertragen. Hierzu wurden zunächst mit Hilfe eines forstbetrieblichen Simulationsmodells (Strugholtz-Englert Modell) eine Grobabschätzung des regionalen Rohholzaufkommens und eine Berechnung von unterschiedlichen Waldbehandlungsoptionen durchgeführt. 
Das Strugholtz-Englert-Simulationsmodell wurde im Rahmen einer Diplomarbeit an der Universität Göttingen entwickelt, um die Auswirkungen von Waldbewirtschaftungsentscheidungen für Forstbetriebe, auf Basis betriebsindividueller Eingangsdaten, im Verlauf einer 200jährigen Simulationsperiode aufzuzeigen (STRUGHOLTZ, 2010). Es wurde im Projekt FFHImpact erfolgreich angewendet, um naturale und ökonomische Veränderungen durch naturschutzfachliche Maßnahmenplanungen zu bewerten. Die Funktionen und Wirkungsweise des Modells sind in Strugholtz (2010) und SeINTSCH et al. (2012) beschrieben. Für die Abschätzung des Holzeinschlags der beiden Untersuchungsregionen sind als Eingangsdaten lediglich die Flächen der Holzartengruppe Eiche, Buche/sonstiges Laubholz, Fichte/Tanne/ Douglasie und Kiefer/Lärche sowie durchschnittliche Umtriebszeiten erforderlich. Dies ist in Regionen mit hohem Privatwaldanteil von Vorteil, da vor allem im Kleinprivatwald häufig keine Informationen auf regionaler Ebene zum Einschlag und zur Verteilung der Altersklassen vorliegen. Im Rahmen der Studie wurden bspw. durchschnittliche Umtriebszeiten (WEHAM-Daten) der Länder Niedersachsen (mit Bremen und Hamburg) und Thüringen unterstellt. Sollten keine weiteren regionalen Angaben zur Altersklassenverteilung, Struktur und Bewirtschaftung der Bestände vorliegen, so können zur Vereinfachung für die Grobabschätzung gleichmäßige Flächenanteile in allen Altersklassen (Normalwald), eine mäßige Durchforstung nach den Ertragstafeln von SCHOBER (1975) und eine flächige Endnutzung nach Erreichen der Umtriebszeit sowie durchschnittliche Bestockungsgrade und Ertragsklassen für alle Baumarten angegeben werden.

\section{Ergebnisse}

Für das Berichtsjahr 2006 wird der FGR-bewertungsrelevante Einschlag der deutschen Forstwirtschaft von DIETER und KÜPPERS (2008:2) auf 67,45 Mio. $\mathrm{m}^{3}$ Rohholz geschätzt. Nicht darin enthalten sind weitere 2,15 Mio. $\mathrm{m}^{3}$ Rohholz für den ausschließlichen Eigenbedarf der Forstbetriebe. Hiermit liegt die FGR-Abschätzung deutlich über der amtlichen Holzeinschlagsstatistik, welche mit 62,3 Mio. $\mathrm{m}^{3}$ den tatsächlichen Einschlag unterschätzen dürfte. Die Bruttowertschöpfung zu Herstellungspreisen der Forstwirtschaft betrug im Jahr 2006 $26,71 € / \mathrm{m}^{3}$. Pro $1.000 \mathrm{~m}^{3}$ Rohholz konnte eine Beschäftigung von 0,64 Vollzeitäquivalenten ermittelt werden. Unter der pauschalen, nicht nach Baumartenanteilen gewichteten Abschätzung eines Rohholzaufkommens von 6,5 m³ Rohholz pro Hektar Holzbodenfläche im Jahr 2006 (FGR-bewertungsrelevantes Rohholzaufkommen von 67,4 Mio. $\mathrm{m}^{3}$ durch eine Holzbodenfläche von 10,3 Mio. m² (FGR 2006)) ergibt sich hier eine Bruttowertschöpfung zu Her- 
stellungspreisen von $174,84 €$ /ha für den Wirtschaftsbereich Forstwirtschaft. Für 100 ha Waldfläche wurde eine Beschäftigung von 0,4 Vollzeitäquivalenten ermittelt.

Auf Grundlage der Analysen von SEINTSCH (2011) und SchweInLE (2012) sind in Tabelle 3 pauschale Abschätzungen zur rohholzbasierten Wertschöpfung und Beschäftigung der Glieder stofflicher Wertschöpfungsketten bis zur ersten Verarbeitungsstufe sowie der energetischen Nutzung (Hausbrand und Hackschnitzel) dargestellt. Wie aus der tabellarischen Darstellung ersichtlich, liegt die Bruttowertschöpfung zu Faktorkosten der stofflichen Nutzung pro Hektar Holzbodenfläche zwischen 74,15€ und 79,81€. Unter der Annahme der Bereitstellung von Waldscheitholz und Hackschnitzeln durch Biomassehöfe wird deutlich, dass die energetische Wertschöpfung, einschließlich der Holzernte, mit 45,57 bis 53,85€ geringer ist.

Tabelle 3: Pauschale Abschätzungen zu Wertschöpfung und Beschäftigung der stofflichen und energetischen Nutzung

\begin{tabular}{|c|c|c|c|}
\hline Wertschöpfungskette & $\begin{array}{c}\text { Bruttowertschöpfung } \\
\text { zu } \\
\text { Faktorkosten } \\
{\left[\text { je } \mathbf{~ m}^{3}\right]} \\
\end{array}$ & $\begin{array}{c}\text { Bruttowertschöpfung } \\
\text { zu } \\
\text { Faktorkosten }^{\text {[je ha] }} \\
\end{array}$ & $\begin{array}{l}\text { Beschäftigte } \\
\text { [je } 1000 \mathrm{~m}^{3} \text { ] }\end{array}$ \\
\hline Forstwirtschaft + Sägeindustrie & $74,15 €$ & $485,46 €$ & 1,41 \\
\hline Forstwirtschaft + Holzwerkstoffindustrie & $77,10 €$ & $504,78 €$ & 1,42 \\
\hline Forstwirtschaft + Holz- und Zellstoffhersteller & $79,81 €$ & $522,51 €$ & 1,04 \\
\hline $\begin{array}{l}\text { Scheitholz/ Hausbrand, einschl. Holzernte } \\
\text { (Bereitstellung über Biomassehof }^{2} \text { ) }\end{array}$ & $53,85 €$ & $352,53 €$ & 0,56 \\
\hline $\begin{array}{l}\text { Waldhackschnitzel / Heizkraftwerke, } \\
\text { einschl. Holzernte } \\
\text { (Bereitstellung über Biomassehof) }\end{array}$ & $45,57 €$ & $298,36 €$ & 0,95 \\
\hline
\end{tabular}

\section{Bezug zu den Untersuchungsregionen}

Die in dieser Wertschöpfungsanalyse im Bundesdurchschnitt errechneten Kennzahlen bieten orientierende Größenordnungen, um die unterschiedlichen Waldnutzungen und Rohholzverwendungsoptionen in den Fallbeispielsregionen einordnen zu können. Hierfür wurden zunächst mit dem Strugholtz-Englert Simulationsmodell die Vorräte und Einschlagsmengen für das Jahr 2013 für die beiden Untersuchungsregionen berechnet und bundesweite Kennzahlen der Bruttowertschöpfung auf den Einschlag in den beiden Untersuchungsregionen übertragen. $\mathrm{Zu}$ beachten ist hier jedoch, dass die Wertschöpfung in den Regionen aufgrund regionalspezifischer Baumartenanteile und Waldbesitzerstrukturen von den bundesweiten Durchschnitts-

\footnotetext{
${ }^{1}$ Annahme: durchschnittlich 6,5 $\mathrm{m}^{3} / \mathrm{ha}$

${ }^{2}$ Diese Wertschöpfungskette gliedert sich in die folgenden Produktions- und Transportschritte: Rundholzernte, Transport zum Biomassehof, stationäre Scheitholzaufbereitung, künstliche Trocknung sowie Transport zum Kunden.
} 
werten abweicht, dass bei den Rechnungen unterstellt wird, dass die Wertschöpfung in der Region verbleibt, und dass der interregionale Holzhandel außer Acht gelassen wurde. Einschlag und Fläche der Baumartengruppen sind in Tabelle 4 dargestellt.

Tabelle 4: Einschlag und Fläche der Baumartengruppen in den Landkreisen Göttingen und BERTA

\begin{tabular}{|l|c|c|c|c|}
\hline & Fläche & \multicolumn{3}{c|}{ Einschlag } \\
\hline & ha & Efm/ha/a & Efm/a & Anteil \\
\hline \multicolumn{5}{|c|}{ Göttingen } \\
\hline Blöße & 669,3 & & 77.365 & $33 \%$ \\
\hline Nadelholz & $27.793,2$ & 6,9 & 157.826 & $100 \%$ \\
\hline Laubholz & $11.093,5$ & 5,7 & 235.191 & \\
\hline Gesamt & 39.556 & 6,0 & & $30 \%$ \\
\hline \multicolumn{5}{|c|}{ BERTA } \\
\hline Blöße & 9,4 & 7,0 & 5.779 & $70 \%$ \\
\hline Nadelholz & 821,1 & 5,2 & 13.418 & $100 \%$ \\
\hline Laubholz & $2.599,0$ & 5,6 & 19.197 & \\
\hline Gesamt & $3.429,5$ &
\end{tabular}

Für die Region Göttinger Land wurde mit dem Strugholtz-Englert-Modell ein Gesamteinschlag von rund $6 \mathrm{Efm} / \mathrm{ha} / \mathrm{a}$ berechnet. Diese Einschlaghöhe weicht vom Einschlag des Landeswaldes im Mehrjahresmittel 2005-2010 von 11,3 m³/ha/a deutlich ab (Kapitel 2.2). Diese Abweichung resultiert aus dem Großschadereignis des Orkans „Kyrill“ im Jahr 2007. Ohne dieses Großschadereignis liegen die Einschlaghöhe in der Modellrechnung und der tatsächliche Einschlag im Landeswald in einer plausiblen und vergleichbaren Größenordnung.

Aufbauend auf dieser Einschlagabschätzung wurden in einem weiteren Arbeitsschritt kontrastierende Szenarien mit den Wertschöpfungsketten „Forstwirtschaft + Sägeindustrie“ und „Hausbrand (Bereitstellung über Biomassehof)“ berechnet. Wenn nicht anders angegeben, wurde eine Nutzung von Laubholz zu $20 \%$ als Stamm- und $80 \%$ als Energieholz sowie Nadelholz zu $80 \%$ als Stamm- und $20 \%$ als Energieholz unterstellt. 
$\underline{\text { Angenommener Status Quo }}$

1. Bundesweiter Verwendungsdurchschnitt (SEINTSCH 2011)

Verwendungsszenarien

2. „Hausbrand“: Nutzung des gesamten Einschlags für den Hausbrand, (z.B. Anstieg der Energiepreise und in der Folge verstärkter Nutzung von Brennholz)

3. Erhöhung des Laubholzanteils der Sägeindustrie (z.B. Förderung der stofflichen Verwendung z. B. neue innovative Laubholzprodukte)

- Laubholz: 50 \% Stammholz, $50 \%$ Energieholz

- Nadelholz: $80 \%$ Stammholz, $20 \%$ Energieholz

\section{$\underline{\text { Waldnutzungsszenarien }}$}

4. „Stilllegung von $5 \%$ der Waldfläche“ (z.B. Umsetzung der Biodiversitätsstrategie).

5. „Stilllegung von Laubaltholzflächen über 120 Jahre“ (z.B. Umsetzung der FFHRichtlinie).

Für die Szenarien 1 bis 3 werden die Flächen und Einschlagsdaten unterstellt, die in Tab. 4 aufgeführt sind. Für die Szenarien 4 und 5 ergeben sich folgende Änderungen: Im Szenario 4 reduzieren sich die Holzbodenfläche der Holzartengruppen um $5 \%$. Somit erfolgt in der Region Göttingen auf 26.404 ha Laubholzeinschlag $\left(149.935 \mathrm{~m}^{3}\right)$ und auf 10.539 ha Nadelholzeinschlag $\left(73.497 \mathrm{~m}^{3}\right)$. In der Region BERTA werden dementsprechend auf 2.469 ha 12.747 $\mathrm{m}^{3}$ Laubholz und auf 780 ha $5.490 \mathrm{~m}^{3}$ Nadelholz eingeschlagen.

Im Szenario 5 werden alle Laubholzflächen über 120 Jahre stillgelegt. In der Region Göttingen werden dementsprechend auf 19.718 ha insgesamt $48.754 \mathrm{~m}^{3}$ und in der Region BERTA auf 2.339 ha $4.388 \mathrm{~m}^{3}$ Laubholz eingeschlagen.

Die Ergebnisse der Szenarienrechnungen für beide Untersuchungsregionen sind Tabelle 5 dargestellt. 
Tabelle 5: $\quad$ Holzbasierte Wertschöpfungsszenarien in den Landkreisen Göttingen und BERTA

\begin{tabular}{|c|c|c|c|c|c|}
\hline $\begin{array}{l}\text { BWS }{ }^{3} \text { bezogen } \\
\text { auf Gesamtwaldfläche }\end{array}$ & 1. Status quo & 2. Hausbrand & $\begin{array}{l}\text { 3. Erhöhung } \\
\text { des Laub- } \\
\text { holzanteils } \\
\text { der Säge- } \\
\text { industrie }\end{array}$ & $\begin{array}{l}\text { 4. Stillle- } \\
\text { gung von } \\
5 \% \text { der } \\
\text { Waldfläche }\end{array}$ & $\begin{array}{l}\text { 5. Stillle- } \\
\text { gung von } \\
\text { Laubaltholz- } \\
\text { flächen über } \\
120 \text { Jahre }\end{array}$ \\
\hline \multicolumn{6}{|c|}{ Landkreis Göttingen } \\
\hline BWS Laubholz [€/ha] & 235,03 & 218,55 & 259,75 & 223,28 & 72,60 \\
\hline BWS Nadelholz [€/ha] & 139,44 & 107,13 & 139,44 & 132,47 & 139,44 \\
\hline BWS $\left[\mathbf{m}^{3}\right]$ & 61,92 & 53,85 & 66,00 & 58,82 & 35,06 \\
\hline BWS gesamt [€/ha] & 374,48 & 325,69 & 399,19 & 355,75 & 212,05 \\
\hline Beschäftigung [Region] & 211,1 & 131,7 & 251,4 & 200,6 & 131,5 \\
\hline \multicolumn{6}{|c|}{ Region BERTA } \\
\hline BWS Laubholz [€/ha] & 227,20 & 211,27 & 251,10 & 215,84 & 74,30 \\
\hline BWS Nadelholz [€/ha] & 118,44 & 90,99 & 118,44 & 112,51 & 118,44 \\
\hline BWS $\left[\mathrm{m}^{3}\right]$ & 61,58 & 53,85 & 65,83 & 58,50 & 34,34 \\
\hline BWS gesamt [€/ha] & 345,64 & 302,27 & 369,53 & 328,35 & 192,74 \\
\hline Beschäftigung [Region] & 17,0 & 10,8 & 20,4 & 16,1 & 10,4 \\
\hline
\end{tabular}

\subsection{Miscanthus und Pappelplantagen im Kurzumtrieb als Alternative zum klassischen Ackerbau}

\section{Problemstellung und Zielsetzung}

In der Dezembersitzung 2010 hat der Kreistag des Landkreises Göttingen beschlossen, dass der Landkreis bis 2040 enerieautark sein soll. Bereits 2025 sollen 50 \% des Stroms aus erneuerbaren Energien bereitgestellt werden. Ein Teil der Energie soll durch die Erweiterung des Anbaus von Biomasse erzeugt werden. In diesem Zusammenhang werden Pappelkulturen im Kurzumtrieb und Miscanthuskulturen mit steigendem Interesse als relevante Alternativen zur Erzeugung regenerativer Energien betrachtet. Dabei ist die Bereitschaft der möglichen Anbauer zur Umstellung noch zurückhaltend. Wir beschreiben die Wirtschaftlichkeit und das Risikoprofil der Kulturen im Vergleich zum konventionellen Ackerbau auf guten Ertragsstandorten, wie z.B. im Landkreis Göttingen. Es wird eine Handlungsempfehlung für einen Anbauer, der sich zwischen der Beibehaltung einer konventionellen Fruchtfolge, bestehend aus Winterweizen, Wintergerste und Winterraps, und dem Anbau von Pappeln im Kurzumtrieb und Miscanthus entscheiden kann, gegeben. Dabei differenzieren wir zwischen der

\footnotetext{
${ }^{3}$ Bruttowertschöpfung
} 


\section{Literatur}

Benoit, C. und B. MAZIJn (Hrsg.) (2010): Guidelines for Social Life Cycle Assessment of Products. United Nations Environment Programme, Paris.

BÖRnER, M.; Guericke, M.; LEDER, B.; NutTO, L. und STÄHR, F. (2003): Erhebung qualitätsrelevanter Parameter am Einzelbaum - Aufnahmestandards für junge bis mittelalte Laubhölzer als Grundlage wissenschaftlicher Untersuchungen. In: Forstarchiv, Jg. 74, S. 275-282.

DIETER, M. und KÜPPERS, J.-G. (2008): Die Forstwirtschaftliche Gesamtrechnung der Bundesrepublik Deutschland 2006. In: Arbeitsbericht, Nr. 2008/1, vTI - Institut für Ökonomie der Forst- und Holzwirtschaft (Hrsg.), Hamburg.

DieTer, M.; Rosin, A. und ThOroe, C. (2004): Die Forstwirtschaftliche Gesamtrechnung der Bundesrepublik Deutschland im Rahmen des ESVG 1995 für die Jahre 1995 bis 2002. In: Arbeitsbericht, Nr. 2004/15, vTI - Institut für Ökonomie der Forst- und Holzwirtschaft (Hrsg.), Hamburg.

Emmann, C. H.; Guenther-LÜBbers, W. und Theuvsen, L. (2013): Impacts of Biogas Production on the Production Factors Land and Labour - Current Effects, Possible Consequences and Further Research Needs. In: International Journal of Food System Dynamics, 4. Jg., H. 1, S. 38-50.

FACHAGENTUR NACHWACHSENDE RoHSTOFFE e.V. (FNR) (Hrsg.) (2012): Energiepflanzen für Biogasanlagen, S. 6, Gülzow.

Finkbeiner, M., E. Schau, A. Lehmann und M. Traverso (2010): Towards Life Cycle Sustainability Assessment. In: Sustainability, 2. Jg., S. 3309-3322.

HENKE, S. (2014): Social Life Cycle Assessment: Multikriterielle Bewertung erneuerbarer Energien, Cuvilier Verlag, Göttingen.

Henke, S. und Theuvsen, L. (2014a): SLCA: Regional differenzierte Bewertung von Biogasanlagen und Kurzumtriebsplantagen. In: Jahrbuch der Österreichischen Gesellschaft für Agrarökonomie 2014 (im Erscheinen).

Henke, S. und Theuvsen, L. (2014b): Social Life Cycle Assessment: Eine sozioökonomische Analyse der Biogasproduktion: In: Schriften der Gesellschaft für Wirtschafts- und Sozialwissenschaften des Landbau e.V., Bd. 49 (im Erscheinen).

Henke, S. und Theuvsen, L. (2013): Social Life Cycle Assessment: Erweiterter Qualitätsbegriff und sozioökonomische Analysemethode. In: Woll, R. und UHLEMANN, R.: Berichte zum Qualitätsmanagement - Vielfalt Qualität- Tendenzen im Qualitätsmanagement, Shaker Verlag, Aachen, S. 271-292.

Husmann, K. (2013a): Prognose ökonomisch optimaler Nutzungsintensität von Buchenkronen. Masterarbeit an der Abteilung für Forstökonomie und Forsteinrichtung, GeorgAugust-Universität Göttingen.

HusmanN, K. (2013b): Auswertung des BEST-Teilprojektes IO-H4 Schwachholzbiomasse, Nordwestdeutsche Forstliche Versuchsanstalt, Göttingen [25.06.2013].

JESSEN, R. J. (1955): Determining the fruit count on a tree by randomized branch sampling. In: Biometrics, 11. Jg., Nr. 1, S. 99-109.

LANDESBETRIEB FÜR STATISTIK UND KOMMUNIKATIONSTECHNOLOGIE NIEDERSACHSEN (2010): Landwirtschaftszählung 2010. Arbeitskräfte in der Landwirtschaft, Leistungen von Lohnunternehmen, Einkommenskombinationen. In: Statistische Berichte Niedersachsen, Nr. 09. [online] http://www.lskn.niedersachsen.de/portal/live.php?navigation_id=25698\&article_id=87561\&_psmand=40. S. 338 [20.06.2013]. 
LANDESBETRIEB FÜR STATISTIK UND KOMMUNIKATIONSTECHNOLOGIE NIEDERSACHSEN (2012): Landwirtschaftszählung 2010. Gemeindeergebnisse Teil I. In: Statistische Berichte Niedersachsen, Nr. 1-A. [online] http://www.lskn.niedersachsen.de/download/75470. S. 22-24 [23.06.2013].

MEHLE, A. (2013): Emailverkehr mit dem Landwirtschaftsamt Sömmerda [5.07.2013].

MÖLDER, I. (2013): Emailverkehr mit der Energieagentur Region Göttingen e.V [15.07.2013].

3N- KOMPETENZZENTRUM NIEDERSACHSEN NETZWERK NACHWACHSENDE ROHSTOFFE (2012): Biogas in Niedersachsen. Entwicklung, Stand und Perspektiven, Niedersächsisches Ministerium für Ernährung, Landwirtschaft, Verbraucherschutz und Landesentwicklung (Hrsg.).

RUMPF, S.; NAGEL, J. und SCHMIDT, M. (2011): Biomasseschatzfunktionen von Fichte, Kiefer, Buche, Eiche und Douglasie für Nordwestdeutschland, Forschungsvorhaben: Möglichkeiten und Grenzen der Vollbaumnutzung. In: Ergebnisbericht (FKZ: 22015407), Fachagentur für Nachwachsende Rohstoffe des Bundesministeriums für Ernährung, Landwirtschaft und Verbraucherschutz (Hrsg), Göttingen.

SABOROWSKI, J. und GAFFREY, D. (1999): RBS, ein mehrstufiges Inventurverfahren zur Schätzung von Baummerkmalen. In: Allgemeine Forst- u. Jagd-Zeitung, 170. Jg., S. 223-227.

SCHOBER, R. (1975): Ertragstafeln wichtiger Baumarten, Frankfurt am Main: J.D. Sauerländer's Verlag.

SchÜTTE, R. (2012): EEG stellt Kulturlandschaft auf den Kopf, S. 4. [online] http://www.lwkniedersachsen.de/index.cfm/portal/6/nav/355/article/19589.html. [21.06.2013].

SCHWEINLE, J. (2012): Wertschöpfungsanalyse der energetischen Nutzung von Holz, in: Arbeitsbericht, Nr. 2012/02, vTI - Institut für Ökonomie der Forst- und Holzwirtschaft (Hrsg.), Hamburg.

SCHWENDIG, F. (2013): Emailverkehr mit dem Landwirtschaftsamt Leinefelde Worbis [4.07.2013].

SeINTSCH, B. (2011): Stellung der Holzrohstoffe in der Kostenstruktur des Holz- und Papiergewerbes in Deutschland. In: Arbeitsbericht, Nr. 2011/03, vTI - Institut für Ökonomie der Forst- und Holzwirtschaft (Hrsg.), Hamburg.

Seintsch, B.; Rosenkranz, L.; Englert, H.; Dieter, M.; WiPPel, B.; Becker, G.; StratMANN, J.; GERST, J. und MÖHRING, B. (2012a): FFH-Impact: Teil 2: Auswirkungen von FFH-Maßnahmenplanungen auf Forstbetriebe. In: Arbeitsbericht, Nr. 2012/05, vTI - Institut für Ökonomie der Forst- und Holzwirtschaft (Hrsg.), Hamburg.

SPEIDEL, G. (1952): Das Stückmassegesetz und seine Bedeutung für den internationalen Leistungsvergleich bei der Forstarbeit. Dissertation, Universität Hamburg.

STATISTISCHE ÄMTER DES BUNDES UND DER LÄNDER (2011): Agrarstrukturen in Deutschland - Einheit in Vielfalt. Regionale Ergebnisse der Landwirtschaftszählung 2010, [online] https://www.destatis.de/DE/Publikationen/Thematisch/LandForstwirtschaft/Landwirts chaftzaehlung/Landwirtschaftliche_Berufsbildung.html. [28.01.2013].

StATISTISCHES BundeSAMT (2012): Daten aus dem Gemeindeverzeichnis. Kreisfreie Städte und Landkreise nach Fläche und Bevölkerung - Gebietsstand: 31.12.2011, [online] https://www.destatis.de/DE/ZahlenFakten/LaenderRegionen/Regionales/Gemeindever zeichnis/Administrativ/Aktuell/04Kreise.xls?_blob=publicationFile [11.02.2013].

STATISTISCHES BundesAmT (StBA) (2013): Agrarstrukturerhebung. [online] https://www.destatis.de/DE/Meta/AbisZ/Agrarstrukturerhebung.html. [11.02.2013]. 
Statistisches Bundesamt (StBa) (2013): Feldfrüchte, [online] https://www.destatis.de/DE/Publikationen/Thematisch/LandForstwirtschaft/ ErnteFeldfruechte/FeldfruechteJahr2030321127164.pdf?_blob=publicationFile [20.06.2013].

StATISTISCHES BundeSAmT (StBA) (2010): Betriebe mit Waldflächen. Landwirtschaftszählung/ Agrarstrukturerhebung. In: Land- und Forstwirtschaft. Fischerei, Fachserie 3, Reihe 2.1.1, S. 11, 20, 27, Wiesbaden. [online] https://www.destatis.de/DE/Publikationen/Thematisch/LandForstwirtschaft/WaldundHolz/BetriebeWaldflaechen.html. [20.06.2013].

StATISTISCHES BundeSAMT (StBA) (2006): Material- und Wareneingangserhebung im Verarbeitenden Gewerbe sowie im Bergbau und in der Gewinnung von Steinen und Erden. In: Qualitätsbericht, Fachserie 4, Reihe 4.2.4, Wiesbaden.

STATISTISCHES BUNDESAMT (StBA): Kostenstrukturerhebung im Verarbeitenden Gewerbe, Bergbau sowie in der Gewinnung von Steinen und Erden. In: Qualitätsbericht, Fachserie 4, Reihe 4.3, Wiesbaden.

StRugholtz, A. (2010): Ein forstbetriebliches Simulationsmodell zur ökonomischen Bewertung strategischer forstlicher Produktionsentscheidungen. Masterarbeit an der Fakultät für Forstwissenschaften und Waldökologie der Georg-August-Universität Göttingen.

Thommen, J. P. und ACHLEITNER, A.-K. (2009): Allgemeine Betriebswirtschaftslehre. Umfassende Einführung aus managementorientierter Sicht. 6.Aufl., Wiesbaden: GablerVerlag.

THÜRINGER LANDESAMT FÜR STATISTIK (TLS) (2010): Anbau auf dem Ackerland von ausgewählten landwirtschaftlichen Fruchtarten nach Kreisen in Thüringen. [online] http://www.tls.thueringen.de/datenbank/ TabAnzeige.asp?tabelle $=\mathrm{kr000515 \% 7C \% 7C}$ [20.06.2013].

Wolbert-Haverkamp, M. (2012): Miscanthus und Pappelplantagen im Kurzumtrieb als Alternative zum klassischen Ackerbau - Eine Risikoanalyse mittels Monte-Carlo Simulation. In: Berichte über Landwirtschaft, Bd. 90, Nr. 2, S. 302-316.

Zschache, U.; von Cramon-Taubadel, S. und Theuvsen, L. (2010): Öffentliche Deutungen im Bioenergiediskurs. In: Berichte über Landwirtschaft, Bd. 88, S. 502-512. 
5. ROSENKRANZ L, SEINTSCH B und DIETER M (2015): Decomposition analysis of changes in value added: a case study of the sawmilling and wood processing industry in Germany. Forest Policy and Economics 54:36-50 


\title{
Decomposition analysis of changes in value added. A case study of the sawmilling and wood processing industry in Germany
}

\author{
Lydia Rosenkranz *, Björn Seintsch, Matthias Dieter \\ Thünen-Institute of International Forestry and Forest Economics, Leuschnerstr. 91, D-21031 Hamburg, Germany
}

\section{A R T I C L E I N F O}

\section{Article history:}

Received 18 August 2014

Received in revised form 19 January 2015

Accepted 20 January 2015

Available online 9 February 2015

\section{Keywords:}

Value added

Decomposition

Wood industry

Paasche Index

Production

Wood input

\begin{abstract}
A B S T R A C T
It is basically assumed that a higher level of domestic wood harvesting leads to a higher level of domestic wood use and thus to an increase of value added in the wood processing industry. However, in the past years, the wood input increased in some wood processing sectors in Germany whereas their value added decreased. Against this background we aimed to decompose changes in input and output in order to isolate the effects of value added and to determine the role of wood as intermediate input of the sawmilling and wood processing industries. We regarded the years 2006 compared to 2002 and 2010 compared to 2006. Based on statistical data, a decomposition method was developed that determines changes in the product price, the intermediate input price, the growth and the structural effect. Furthermore, a special focus was set on the impacts of price and quantity changes of the input of wood-based products. We found out, that the growth effect is connected to the wood input in the sawmilling industry in the considered years. Also, it became apparent that a higher input of wood and wood products does not automatically lead to an increase in value added.
\end{abstract}

C 2015 Elsevier B.V. All rights reserved.

\section{Introduction and research objective}

There is a prevailing understanding that a higher or lower level of domestic harvesting and utilisation of wood induces a higher or lower level of value added in the wood-based industry (e.g., Dieter, 2008). The wood charter of the German Federal Government, for instance, aimed for a $20 \%$ increase of the domestic per-capita consumption of sustainably produced wood and wood products, and thus, amongst others for an increase in value added in rural regions (BMELV, 2004).

It is also basically assumed that a higher or lower material input of wood automatically leads to a higher or lower material output of wood products and value added in the wood-based industry. In reality a bundle of factors (e.g., availability of raw materials, substitutional effects and technological improvements) influences input, output and changes of value added. Therefore, for the single industries of the wood-based sector, a general causal link between wood-based input, output and value added is not detectable. Some sectors even show an opposite direction between the development of wood input and the development of value added: whereas wood input is increasing, the value added is decreasing (StBA: F 4 R 4.3; StBA Arbeitsunterlage Rohholz, Mantau, 2012; Seintsch, 2013).

As to the question on the connection between (wood-based) input, output and value added Sinn (2005) provides a possible explanation.

\footnotetext{
* Corresponding author. Tel.: +49 4073962 313; fax: +49 4073962399.

E-mail addresses: lydia.rosenkranz@ti.bund.de (L. Rosenkranz),

bjoern.seintsch@ti.bund.de (B. Seintsch), matthias.dieter@ti.bund.de (M. Dieter).
}

According to him, two general trends could be noted in German industrial enterprises in the past few years: an increase in 1) offshoring, i.e., shifting of labour-intensive parts of value added chains to branches abroad and in 2) foreign outsourcing, i.e., buying of material and commodities from foreign suppliers and therefore both avoiding the expensive German labour-costs. Based on this finding, Sinn (2005: 6) developed the "Bazaar-Hypothesis" which states that "the foreign share of value added of the industrial production, the so called vertical integration, declines in favour of foreign countries and that Germany increasingly specialises in "Bazaar-Activities"'. He explains, that between 1995 and 2003 four fifth of the decline of value added can be explained by foreign and about one fifth by domestic outsourcing and offshoring. Thus, according to Sinn (2005) an increase in the share of intermediate input and a decrease in the share of value added in a national economy mean to a large extent an outsourcing to foreign economies.

Dieter $(2005,2010)$ analysed the Bazaar-Hypothesis for the foreign trade with wood and wood-based products of the German economy. Dieter (2005) observed an increase in the balance of foreign trade for timber and timber products. He tested the "Bazaar-Hypothesis" in regard to the timber market by means of input-output-tables for the years 1993 and 2006 and also detected a slight decrease of vertical integration. However, he explained that "within a single sector a decrease of value added could also mean a shifting of intermediate input by means of domestic outsourcing" (Dieter, 2005: 7). Also Dieter (2010) analysed input-output tables for 2004 and discovered mostly domestic outsourcing in the case of the wood-based industry, meaning that production value remains largely within Germany. He explained that outsourcing may also signify a concentration on the core-business 
of the enterprise and an intensification of labour division, helping enterprises to specialise and thus to secure and enhance sales quantities Dieter (2010: 39).

Against this background, we aim to decompose the changes in input and output in order to isolate the effects on value added. We consider changes in product prices, intermediate input prices, growth and structural changes. A special emphasis is put on the effects of price and quantity changes in wood input. As two case study objects we use the economic sectors ES 1610 "Sawmilling and planing of wood" and ES 1621 "Veneer sheets and wood-based panels", both major players in the wood-based industry. We regard the time period between 2002 and 2010. For our purpose a method, based on statistical data, was developed and applied to ES 1610 and ES 1621.

Within the paper, the following research questions shall be discussed:

1. To what extent can the effects be attributed to changes in value added?

2. What is the influence of the intermediate input group "raw wood" and other wood-based commodities on changes in value added?

\section{Literature review}

So far, diverse methods of a decomposition of effects were used for different purposes of analysis. In general, decomposition studies seek to analyse the effects of single elements of changes in e.g., economic growth, competitiveness, energy consumption or employment over a defined period of time.

Klein et al. (2009), for example, determined regional changes of employment trends of the German wood-based industry, decomposed into national trends, sectoral trends and regional conditions by means of a shift-share analysis. Sakamoto and Fan (2009) examined the disparity of regional income in China, based on value-added data. They focussed on the aspect of distribution which was decomposed into employee's wages, depreciations, operating surplus and net tax on production using a population weighted coefficient of variation.

The industrial economic growth in Bulgaria was examined by Ivanov and Webster (2010). They identified three existing approaches of decomposition for their purpose: the decomposition of the contribution of production factors, the decomposition of types of expenditures and the decomposition of the role of single industries to economic growth. For their study they extended the third approach in order to create the possibility of inter-industrial comparison, by using the gross domestic product as well as the gross value added of industries. Another approach, this time for productivity change, was developed by O'Donnell (2012) by using an aggregate quantity framework to decompose index numbers of total factor productivity "into measures of technical change, technical efficiency change, mix efficiency change and scale efficiency change" (O'Donnell, 2012: 269).

In regard to the determination of competitiveness by means of decomposition Dieter and Englert (2007, 2009), for example, analysed the competitiveness of the global wood industry sector, and especially on Germany. They analysed the growth of export of timber and timber products, amongst others, based on the formula of constant market share analyses (CMS) developed by Milana (1988). In this study, the "periodical change in a country's overall exports is divided into [...] (1) the world growth effect, (2) the commodity-composition effect, (3) the market distribution effect and (4) the residual, which is usually interpreted as the competitiveness effect" (Dieter and Englert, 2007: 407).

The method of decomposition is also often used to identify factors of change in energy and environmental indicators. In 1995, Ang (1995) already identified 51 studies featuring decomposition analyses in the energy sector. Ang et al. (1998) used a logarithmic mean weight function for a complete decomposition of changes of energy demand and gas emissions and applied it on case studies for China, Korea and
Singapore. Sun and Ang (2000) developed decomposition models by extending basic models by Laspeyres, Paasche and Marshall-Edgeworth in order to eliminate residuals and tested them on a case study on energy-related $\mathrm{CO}_{2}$-emissions in 15 European countries.

In conclusion, although numerous studies have been conducted, using the decomposition approach in various fields, so far there is no analysis dealing with the effects of changes of production on the value added, especially in the wood-based sector. In contrast to the abovementioned studies, our paper therefore focuses on the decomposition of changes in value added of the economic sectors ES 1610 "Sawmilling and planing of wood" and ES 1621 "Veneer sheets and wood-based panels" in Germany in order to understand its driving factors and the influence of wood input in particular.

\section{Material and methods}

\subsection{Decomposition analysis}

As discussed above, an increase in product output, ceteris paribus, leads to an increase in value added. However, according to economic theory, an increase in product output also leads to an at least marginal decrease in product prices and an at least marginal increase in input prices. Also, there are other factors which have an impact on input and output prices and changes value added.

Indeed, as mentioned before, for the past years the economic sectors ES 1610 and ES 1621 in Germany have lost value added shares referred to total output value for the benefit of (i) domestic intermediate input (domestic outsourcing) and imports of (ii) intermediate input from abroad (foreign outsourcing) and (iii) products through removal of own production capacities to foreign countries (offshoring).

Taking these considerations into account the (nominal) value added can basically change between two periods or years due to four reasons:

I Change in (nominal) product prices

II Change in (nominal) prices of input goods for intermediate consumption (in the following called intermediate input)

III Change in output of products

IV Change in shares of intermediate input and value added due to outsourcing and offshoring in one direction or vertical integration in the other.

These four reasons can be mathematically formulated as the four elements of change in value added as shown in Eq. (1).

$\Delta \mathrm{VA}=\mathrm{I}+\mathrm{II}+\mathrm{III}+\mathrm{IV}$

With

$\mathrm{I}=\mathrm{X}_{1}^{\mathrm{G}} * \mathrm{P}_{1}^{\mathrm{G}}-\mathrm{X}_{1}^{\mathrm{G}} * \mathrm{P}_{0}^{\mathrm{G}}$

$\mathrm{II}=\mathrm{X}_{1}^{\mathrm{V}} * \mathrm{P}_{0}^{\mathrm{V}}-\mathrm{X}_{1}^{\mathrm{V}} * \mathrm{P}_{1}^{\mathrm{V}}$

$\mathrm{III}=\left(\mathrm{X}_{1}^{\mathrm{G}} * \mathrm{P}_{0}^{\mathrm{G}}-\mathrm{X}_{0}^{\mathrm{G}} * \mathrm{P}_{0}^{\mathrm{G}}\right) *\left(1-\mathrm{S}_{0}^{\mathrm{V}}\right)$

$\mathrm{IV}=\mathrm{S}_{0}^{\mathrm{V}} * \mathrm{X}_{1}^{\mathrm{G}} * \mathrm{P}_{0}^{\mathrm{G}}-\mathrm{X}_{1}^{\mathrm{V}} * \mathrm{P}_{0}^{\mathrm{V}}$

and

$\mathrm{S}_{0}^{\mathrm{V}}=\frac{\mathrm{X}_{0}^{\mathrm{V}} * \mathrm{P}_{0}^{\mathrm{V}}}{\mathrm{X}_{0}^{\mathrm{G}} * \mathrm{P}_{0}^{\mathrm{G}}}$

VA value added

$\mathrm{X} \quad$ quantity of goods

P price

G output

$\mathrm{V} \quad$ intermediate input

0 base year

1 reporting year

$S^{\mathrm{V}} \quad$ share of intermediate input referred to total output value. 
In line with the theoretical derivation above the elements shall be called as

I product price effect

II intermediate input price effect

III growth effect

IV structural effect.

As Appendix A.1 shows Eq. (1) is complete and consistent. The change in value added, after resolution of the four terms I to IV, corresponds with the basic definition of value added: the change in output value less the respective intermediate input value. Since price effects are accounted for in terms I and II, the following (III) growth and (IV) structural effects refer always to the prices of the period before, which is the base period in a time series analysis for more than two periods. Usually, years are elected to be the period.

In addition to Eq. (1) the decomposition can also be constructed in geometrical form. Fig. 1 visualises the four effects mentioned above. It is obvious that the change in value added results from the gains in product output and product prices (full coloured areas I and III.a) less the gains in intermediate input and input prices (hatched areas II, III.b and IV). The price effects of product (areas I) and intermediate input (area II) prices are easy to grasp. This does, however, not apply for the growth effect. The growth effect has to be disaggregated into a positive term resulting from the term in left brackets times 1 (III.a) and a negative term resulting from the respective multiplication with the share $S_{0}^{V}$ (III.b) (see Eq. (1)). As Appendix A.2 shows III.b is exactly the share of $\mathrm{S}_{0}^{\mathrm{V}}$ of III.a. Bearing this in mind, the structural effect can be deduced from the elements of term IV.

The decomposition of the change in value added as presented in Eq. (1) and Fig. 1 can be deemed to be in accordance with the idea behind the Paasche-Index (Frenkel and John, 1999: 118 f), but with a subtraction instead of a division. This approach was chosen for our study since it allows focusing on mere quantity effects as done in terms III and IV of Eq. (1). This is the reason why no alternative according to the idea behind the Laspeyres-Index (Frenkel and John,

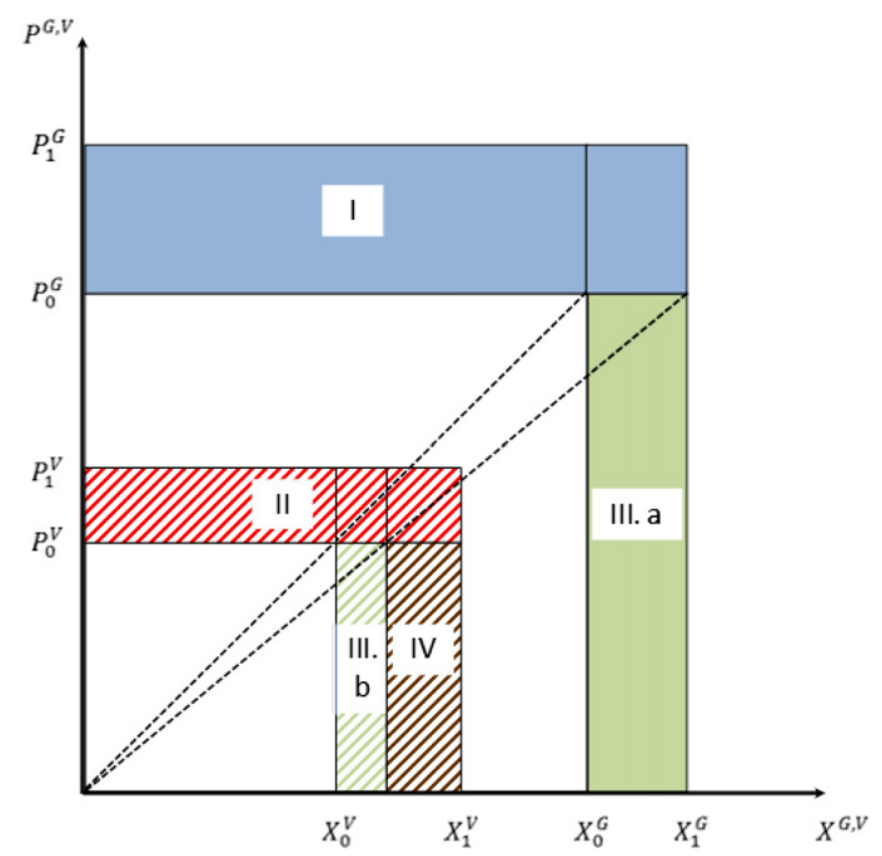

Fig. 1. Schematic and exemplary depiction of the four effects of value added change according to Eq. (1) (full coloured areas indicate positive signs, hatched areas indicate negative signs).
1999: 118 f) was developed. However, we have to accept that the intersections of I and III.a as well as of II, III.b and IV also comprehend gains due to quantity effects which could also be attributed to the respective columns.

\subsection{Determination of quantity and prices}

In our study we aimed to determine the dimension of the I) product price effect, II) the intermediate input price effect, III) the growth effect and IV) the structural effect for the economic sectors ES 1610 "Sawmilling and planing of wood" and ES 1621 "Veneer sheets and wood-based panels", for the year 2006 compared to 2002 and 2010 compared to $2006^{1}$.

For this, prices and quantities for input as well as output of the considered economic sectors needed to be determined for the years 2002, 2006 and 2010. In regard to the input data, the cost structure statistics (StBA F4 R4.3) as well as the material and commodity input statistics (StBA F4 R4.2.4) offer absolute values of the gross production value and selected intermediate input positions. Producer price indices can be taken from the official price statistics (StBA F16 R1; F17 R2). Based on this data, the changes of price and quantity for the decomposition analysis were calculated as follows.

In a first step the gross production value was determined for the three years based on the cost structure statistics (StBA F3 R 4.3). In these statistics the gross production value is defined as the turnover of an enterprise from sales of goods and rendering of services, plus the revenue from commodities and wholesale trade, the revenue from other nonindustrial or non-mechanical services and the changes in inventories of self-produced finished goods, work in progress and capitalised production (StBA F4 R4.3). We assume that the gross production value is the product of quantity and price of the branch, which is in turn the sum of the product of quantity and price of the specific output of the branch and that other effects (e.g., changes in inventory) can be neglected (Eq. (2)). This assumption is based on the fact that StBA F4 R4.3 differentiates the gross production value into turnover from 1.) companies' products and rendition of services, 2.) trade goods and wholesale trade and 3.) other activities.

$\mathrm{X}_{\cdot}^{\mathrm{G}} * \mathrm{P}^{\mathrm{G}}=\sum_{\mathrm{i}=1}^{\mathrm{n}} \mathrm{X}_{\mathrm{i} .}^{\mathrm{G}} * \mathrm{P}_{\mathrm{i} .}^{\mathrm{G}}$

$\begin{array}{ll}\text { X } & \text { quantity of goods } \\ \text { P } & \text { price } \\ \text { G } & \text { output } \\ \text { i } & \text { specific output product of the branch }\end{array}$

The growth factors of prices for 2002, 2006 and 2010 were then calculated based on price indices from the price statistics (StBA F 17 R2). This was done for the two economic sectors for the production value and the respective total intermediate input.

As base years 2002 and 2006, respectively, were chosen. As a basis for the calculation of the growth factors of the gross production value we assume, that the output of ES 1610 is determined by goods of the product group 161 "wood sawn and planed" and ES 1621 by goods of the product group 1621 "veneer sheets and wood-based panels". Therefore, the growth factor of the gross production value for each economic sector was calculated based on the price indices of these two product groups.

As only the revenue and the growth factor of the output prices were determinable from the statistics the value of one unit of input was determined to be $1 €$. With this setting the quantity of output goods equals the output revenue of the base year. The quantity of output goods of the

\footnotetext{
1 These years were determined by the quadrennial appearance of the material and commodity input statistics (StBA F3 R 4.2.4) as a basis for the calculation of the effects II to IV.
} 
reporting years could then easily be determined as the quotient of the gross production value and the growth factor of the price of the respective year.

In a next step, the growth factor of price and quantity of the total intermediate input were identified. The total intermediate input is defined as the sum of quantity and price of all the specific input goods of the branch (Eq. (3)).

$$
\mathrm{X}^{\mathrm{V}} * \mathrm{P}^{\mathrm{V}} \cdot=\sum_{\mathrm{j}=1}^{\mathrm{m}} \mathrm{X}_{\mathrm{j} .}^{\mathrm{V}} * \mathrm{P}_{\mathrm{j}}^{\mathrm{V}}
$$

$\begin{array}{ll}X & \text { quantity of goods } \\ \text { P } & \text { price } \\ V & \text { intermediate input } \\ \text { j } & \text { specific input product of the branch }\end{array}$

By its definition it becomes clear that the intermediate input consists of many different product groups plus costs for services, rents and leases and other costs. In the cost structure statistics the intermediate input is differentiated in raw materials and operating supplies, energy, commodities, costs for hired labour, costs for other industrial and mechanical services, costs for temporary workforces, rents and leases, other costs, taxes other than on income and interest expenses. Raw wood and wood products as major intermediate input positions of both economic sectors are subsumed along many others in the group "consumption of raw materials and operating supplies (materials)". This group has a share of $50-70 \%$ of the gross production value within both sectors. However, this group is not further differentiated. Due to the many different intermediate input positions within this group, growth factors for quantity and prices of the intermediate input as a whole can yet only be vaguely estimated. The cost structure statistics (StBA F3 R 4.3) only provides rough information in regard to intermediate input and is thus not sufficient for the calculation of a weighted average of growth factors of prices. Also, it does not contain information on the influence of raw wood and wood-based products within the cost structure of the economic sectors.

\subsection{Determination of weighted average growth factors of the intermediate input}

In order to calculate a weighted average growth factor of the price of the total intermediate input for each sector, the value of each input good is needed. Thus, for a determination of a more differentiated database of the single intermediate input positions, we used the approach developed by Seintsch (2011b: 33f.) integrating the material and commodity statistics into the cost structure statistics (StBA: F4 R4.3; F4 R4.2.4). This approach of integration is enabled by the same statistical report category and the identical registration units of both statistics. The sum of "receipt of raw materials and supplies" and "receipt of commodities" of the cost structure statistic equals the "total receipt of material and commodities" of the material and commodity input statistics. Based on this connection and taking changes in inventory into account an integration of both statistics is possible and the expenditure for each intermediate input position can be calculated for each respective year.

In a next step, a price index of the StBA was assigned to each product group as well as to all other intermediate input (costs for hired labour, costs for other industrial and mechanical services, costs for temporary workforces, rents and leases, other costs). When price indices were not available for a product group, dummy data (e.g., lignite for "solid fuels") or the average German producer price index for the respective year was used. Average growth factors of price, weighted on their respective base year for 2002, 2006 and 2010 for all intermediate input positions could now be calculated.
Based on these results, the weighted average growth factor of the quantity of the input goods could be determined in the same way as for the output quantity mentioned above. Also, by means of the quotient of the growth factors of input value and price the growth factors of quantity for each input good/service could be calculated.

As a side effect of the integration of material and commodity statistics into the cost structure statistics, growth factors of price and quantity of the various components of the intermediate input (e.g., raw wood or other wood-based input groups) could now be calculated. These are a valuable basis for the interpretation of the results of the decomposition analysis.

\subsection{Data base}

As a data base for our calculation statistics from the Federal Statistical Office of Germany (StBA) were used. The cost structure statistic (F 4 $\mathrm{R} 4.3$, StBA) provides information on production results, production factors as well as the value added in its different gradations. This annual statistic covers all enterprises with 20 or more employees. The enterprises are assigned to the economic branch depending on which of their activities generates the most value added (main activity). In consequence, although an enterprise may be assigned to a specific economic branch due to its main activity, it could still be generating value added through other economic activities outside the main activity. Included in the statistical inquiry are enterprises within the federal territory of Germany and with all producing and non-producing parts.

The quadrennial material and commodity input statistics (F 4 R 4.2.4, StBA) specifies the distribution of intermediate input used in the branches of production and provide information on the interdependence in regard to goods of the economic branches. It covers enterprises with 20 or more employees and whose main activity lies within these sections and who are situated in Germany. The material and commodity input statistics list all raw materials and supplies with acquisition costs, used for working and processing within the enterprise or which have been passed to other enterprises for that reason. This includes fuels, energy, packaging materials, trade commodities and supplies for company kitchens and canteens. In the material and commodity input statistics (StBA F4 R 4.2.4) the receipts of goods are systemised according to the "Classification of Material and Goods Received in Manufacturing, Mining and Quarrying" (WE2002 and WE2010). This classification is based largely on the "Product Classification for Production Statistics" (GP2002 and GP2009).

The average yearly price indices were taken from the price index for industrial products (StBA F17 R2) and the price index "costs for labour" (StBA F 16 R 2.2). The price index for industrial products (StBA F17 R2) representatively measures the development of prices for commodities produced and sold by energy- and water industries, mining and processing trade in Germany on a monthly basis. Overall indices as well as indices for a large number of goods at various levels of aggregation are available. The overall index is the sum of all industrial domestic turnovers as stated in the monthly report of mining and processing trade (StBA F 4 R 4.1.1). The individual price series are based on monthly price surveys of about 5200 enterprises and representative 1260 kinds of goods. The indices are calculated using the Laspeyres formula. The current base year is 2010. As the producer prices of the price statistics is also systemized based on the "Product Classification for Production Statistics" a high consistency in our data is given. The price index "costs for labour" (StBA F 16 R 2.2) collects data from about 40,500 enterprises (sample size). It shows the number of full-time, part-time or marginally employed jobholders, their working hours and their gross income. The annually appearing survey includes industry and service branches with 10 or more employees and classifies the data for the individual German economic sectors of section B to $\mathrm{S}$. Appendix $C$ shows the data bases of the price indices of the intermediate input positions. 


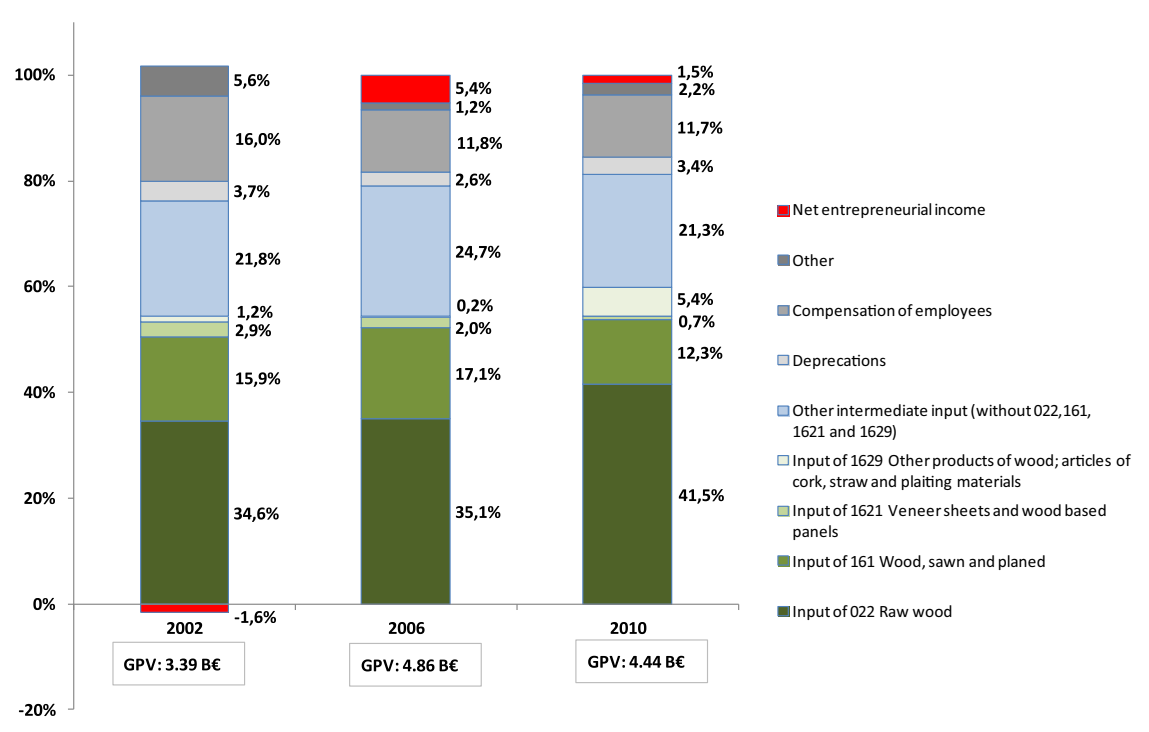

Fig. 2. Share of net entrepreneurial income, compensation of employees, depreciations, and intermediate input of ES 1610 for the years 2002 , 2006 and 2010 (StBA F4 R3.4; StBA F4 R4.2.4). ( $\mathrm{GPV}=$ gross production value).

\section{Results}

4.1. Share of selected intermediate input positions of the gross production value

For the later decomposition analysis the material and commodity input statistics was integrated in the cost structure statistics. For a classification of the results of the decomposition analysis and of the changes of quantity and price of the single intermediate input positions, the results of this integration are shown in Figs. 2 and 3. The figures give an overview of the share of net entrepreneurial income, compensation of employees, depreciations, input of the product groups 022 "raw wood", 161 "wood sawn and planed", 1621 "veneer sheets and woodbased panels" and other costs and other intermediate input in gross production value (StBA F4 R3.4, StBA F4 R4.2.4).

In the economic sector ES 1610, an increase in total gross production value from 3.39 billion $€(B €)$ in 2002 to 4.86 B€ in 2006 , followed by a decrease to $4.44 \mathrm{~B} €$ in 2010 was noted. The difference of the gross value added between 2002 and 2006 was 240.345 T€ and from 2006 to 2010
-202.034 T€. Accordingly, the share of gross value added of the gross production value declined from $24 \%$ in 2002 to $19 \%$ in 2010 . The share of intermediate input of the gross production value increased from $76 \%$ in 2002 to $79 \%$ in 2006 and to $81 \%$ in 2010 . With a share of more than $53.4 \%$ the product groups 022 "raw wood", 161 "wood, sawn and planed" and 1621 "veneer sheets and wood-based panels" represent a considerable part of the gross production value. The share of input of 022 "raw wood" of the gross production value rose from $34.6 \%$ in 2002 to $41.5 \%$ in 2010. The input of 161 "wood, sawn and planed" increased from $15.9 \%$ in 2002 to $17.1 \%$ in 2006 and declined to $12.3 \%$ in 2010. Only the share of 1621 "veneer sheets and wood-based panels" declined by roughly $2 \%$ in these years. In 2002 the costs for intermediate input, depreciations, compensation of employees and other costs exceeded the gross production value, leading to a negative net entrepreneurial income of $-1.6 \%$ in ES 1610 .

In the economic sector ES 1621, the total gross production value increased from $4.38 \mathrm{~B} €$ in 2002 to $5.19 \mathrm{~B} €$ in 2006 and decrease to $4.86 \mathrm{~B} €$ in 2010. The difference of the gross value added between 2002 and 2006 was $-151.889 \mathrm{~T} €$ and from 2006 to $2010-247.532 \mathrm{~T} €$. In ES 1621, too,

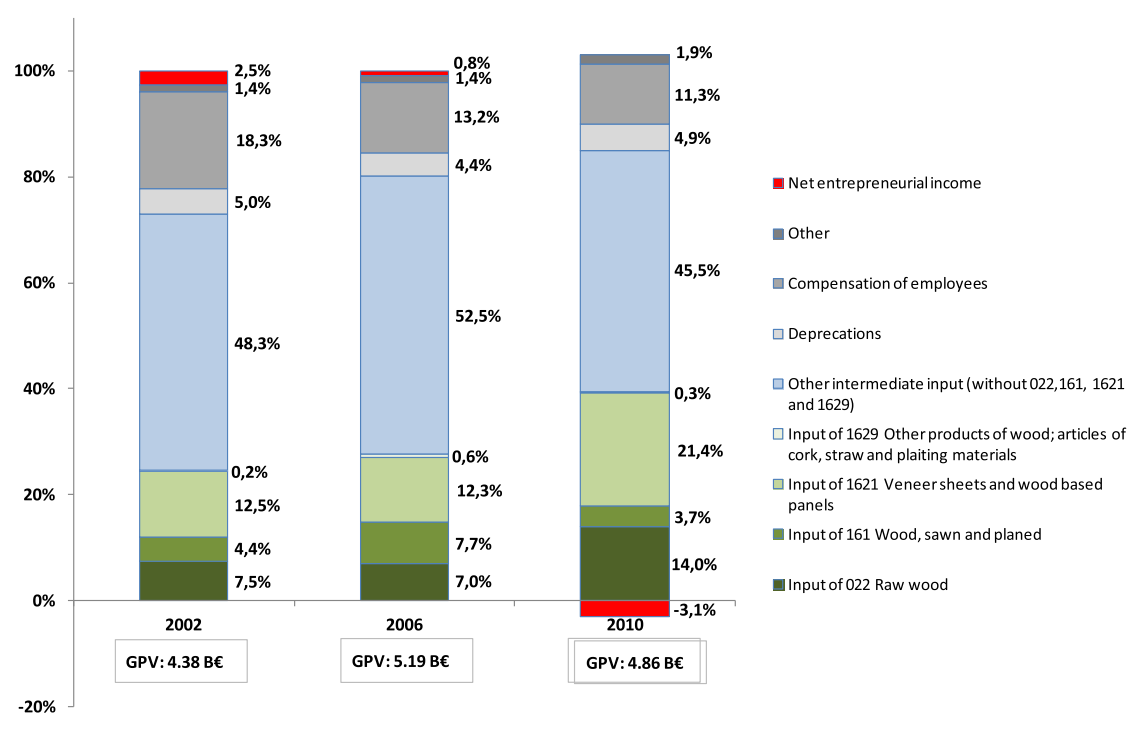

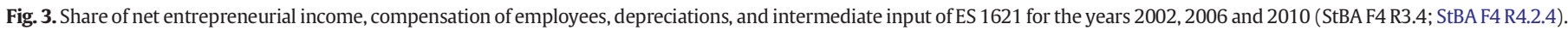


the share of gross value added of the gross production value declined. Compared to the year 2002 the share of gross value added in the year 2010 was reduced by one third. The share of intermediate input in gross production value increased from $73 \%$ in 2002 to $80 \%$ in 2006 and to $83 \%$ in 2010.

In ES 1621 the input goods 022 "raw wood", 161 "wood, sawn and planed" and 1621 "veneer sheets and wood-based panels" represent only $24.4 \%$ in 2002 to $40 \%$ in 2010 of the gross production value. The share of 1621 "veneer sheets and wood-based panels" (12.5\% to $21.4 \%$ ) and 022 "raw wood" (7.5\% to $14 \%$ ) almost doubled between 2002 and 2010, whereas the share of 161 "wood, sawn and planed" rose from $4.4 \%$ in 2002 to $7.7 \%$ in 2006 and declined to 3.7\% in 2010. As in ES 1610 in 2002 the enterprises of ES 1621 experienced a loss of $3.1 \%$ the share of the net entrepreneurial income of the gross production value in the 2010 .

In conclusion, the share of intermediate input of the gross production value increased in both economic sectors. Wood-based product groups, with a share of roughly two thirds of the total intermediate input, play a major role in ES 1610. In ES 1621, however, with a share of roughly one third, they have smaller significance.

\subsection{Growth factors of price and quantity of the intermediate input}

In order to calculate weighted average growth factors of price and quantity of the intermediate input and to provide further explanations of the results of the decomposition analysis, the growth factors of price and quantity were determined for all individual intermediate input positions. The total intermediate inputs, as well as the growth factors of price and quantity of the single intermediate input positions of ES 1610 and ES 1621, are shown in Appendices D.1 and D.2. Regarding the growth factors of price and quantity of the individual intermediate input positions their strongly varying shares within the cost structure of the economic sectors must be kept in mind.

Fig. 4 shows the growth factors of price and quantity for selected input items of ES 1610 for the year 2006 compared to 2002. On first glance the great range of growth factors of the quantity input (between
0.2 and 2.0 of the year 2006) becomes apparent. Approximately half of the intermediate input positions are characterised by a decrease, the other half by an increase in quantity. The relative range of the growth factors of the prices of the individual intermediate input positions lies between 1.0 and 1.20. The growth factors of the price of the product groups 022 "wood in the rough" and 161 "wood, sawn and planed" was 1.067 and 1.141 and the growth factors of the quantity were 1.361 and 1.349. Based on the differentiated calculations of the individual intermediate input positions, an overall growth factor of the price of 1.102 and an overall growth factor of the quantity of 1.35 were determined for the year 2006. Deleted as outliers in Fig. 4 were the input positions 1623 "other builders' carpentry and joinery" (growth factor of price: 0.926 , growth factor of volume: 5.342), 171 "pulp, paper and paperboard" (growth factor of price: 0.947, growth factor of volume: 2.672 ) and "costs for temporary workforces" (growth factor of price: 1.035 , growth factor of volume: 4.661 ). The relative share of these intermediate input positions of the gross production value for 1623 "other builders' carpentry and joinery" was $2.9 \%$, for 171 "pulp, paper and paperboard" $0.1 \%$ and for "costs for temporary workforces" $0.5 \%$.

Fig. 5 shows the growth factor for the year 2010 compared to 2006. When comparing the year 2010 to 2006 a relative decrease of quantity of almost all intermediate input positions becomes visible. The growth factors of the quantity of the intermediate input positions lie for the largest part between $0.10 \%$ and $1.0 \%$. The growth factor of the quantity of 022 "wood in the rough" was 0.854 and of 161 "wood, sawn and planed" 0.612 . In contrast, the growth factor of the price of the product groups 022 "wood in the rough" and 161 "wood, sawn and planed" was 1.264 and 1.075. Based on these results the growth factor of the price and quantity of the total intermediate input for the year 2010 was 1.15 and 0.813 . Deleted as outliers in Fig. 5 were 935 "district heating (steam) and compressed air" (growth factor of price: 1.116, growth factor of volume: 29.758) and 1629 "other products of wood; articles of cork, straw and plaiting materials" (growth factor of prices: 1.028, growth factor of volume: 20.295 ) with a share of the gross production value of $0.3 \%$ and $5.4 \%$, respectively. The latter could be explained by the change of the classification of the economic sectors from WZ 2003
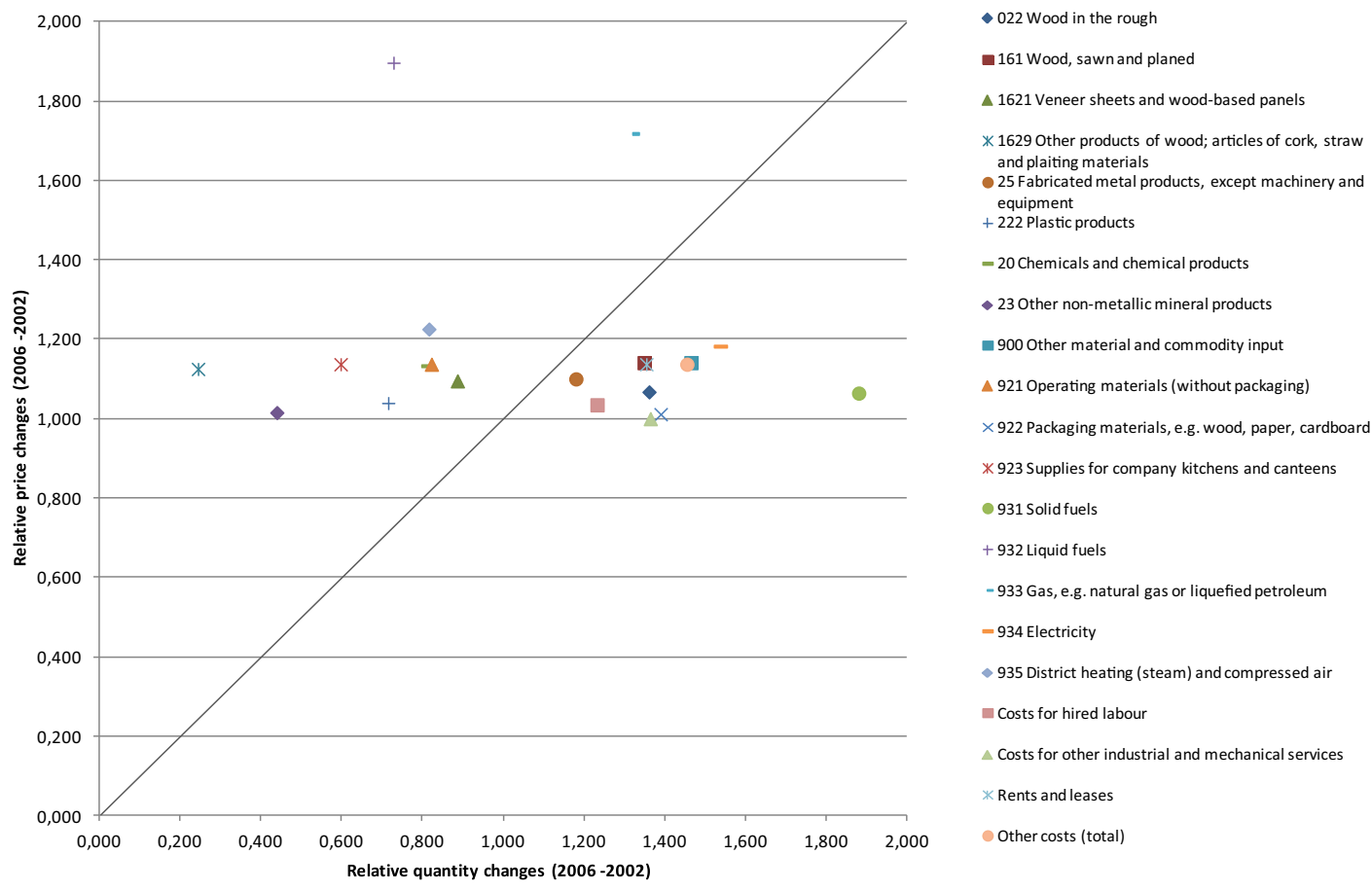

Fig. 4. Relative changes in price and quantity of the intermediate input of ES 1610 "Sawmilling and planing of wood" for the year 2006 compared to $2002 .^{\text {a }}$

${ }^{a}$ For a better visibility all intermediate input with an increase of quantity or price higher than two were deleted in Figs. 4-7. These input positions appear in Appendix D.1. 

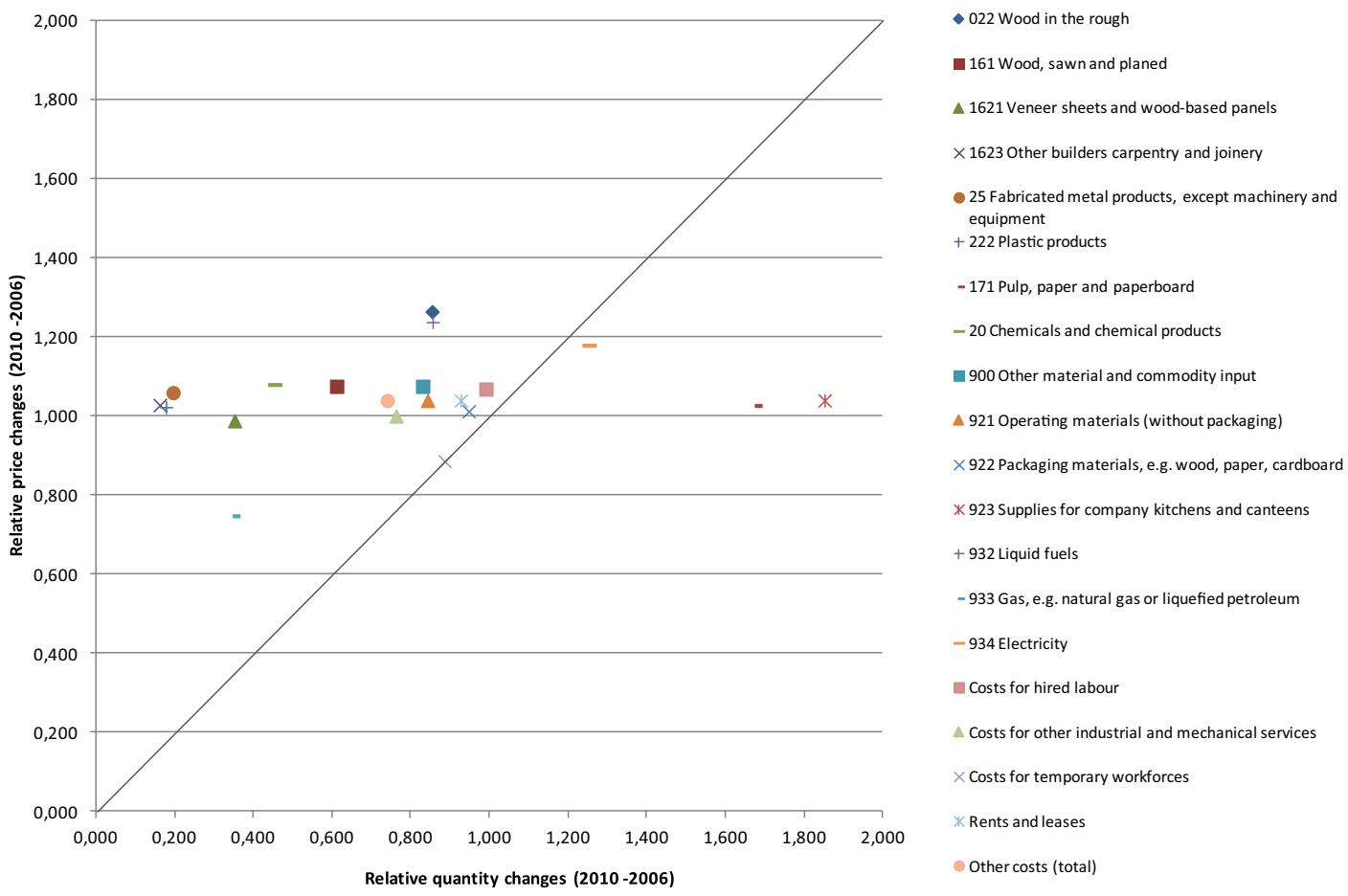

Fig. 5. Relative changes in price and quantity of the intermediate input of ES 1610 "Sawmilling and planing of wood” for the year 2010 compared to 2006.

to WZ 2008, in which pellets and other woody fuels were assigned from the 1621 "Wood sawn and planed" to 1629 "other products of wood; articles of cork, straw and plaiting materials".

Fig. 6 shows the growth factors of prices and quantities for selected input items of ES 1621 for the year 2006 compared to 2002. It can be seen that a large part of the intermediate input positions only shows a small change of quantity (between 0.8 and 1.4). As the same price indices as in ES 1610 were implied, the growth factors of the prices in the intermediate input of this economic sector also lie between 1.0 and 1.2. The growth factors of the prices of the product groups 022 "wood in the rough" and 161 "wood, sawn and planed" were 1.067 and 1.141. The growth factor of quantities was 1.048 and 1.812 . The weighted average growth factor of prices and quantities of the total intermediate input for the year 2006 was 1.108 and 1.164. In Fig. 6 the input positions 1629 "other products of wood; articles of cork, straw and plaiting materials" (growth factor of price: 1.125, growth factor of
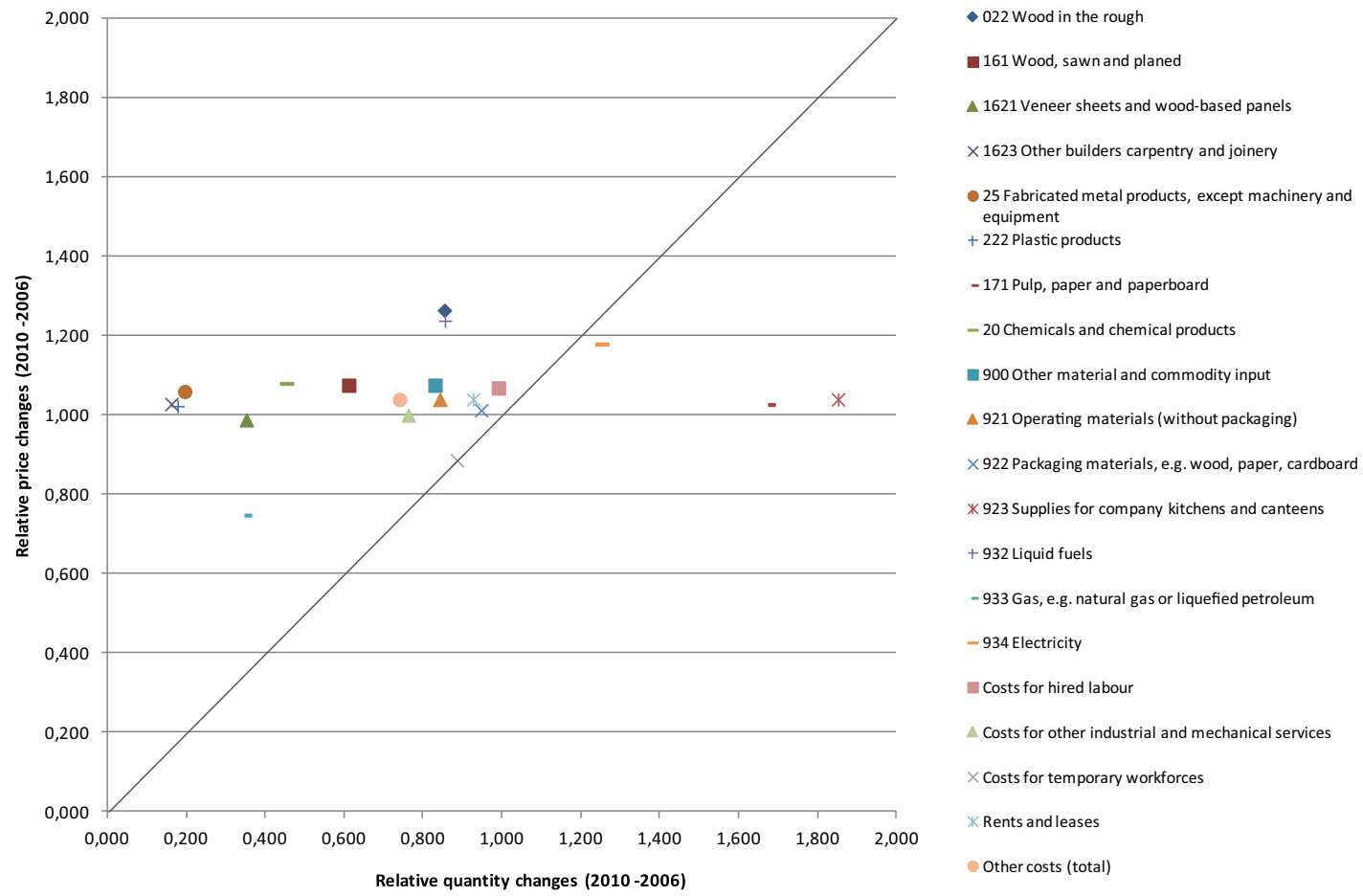

Other costs (total)

Fig. 6. Relative changes in price and quantity of the intermediate input of ES 1621 "Veneer sheets and wood-based panels" for the year 2006 compared to 2002. 


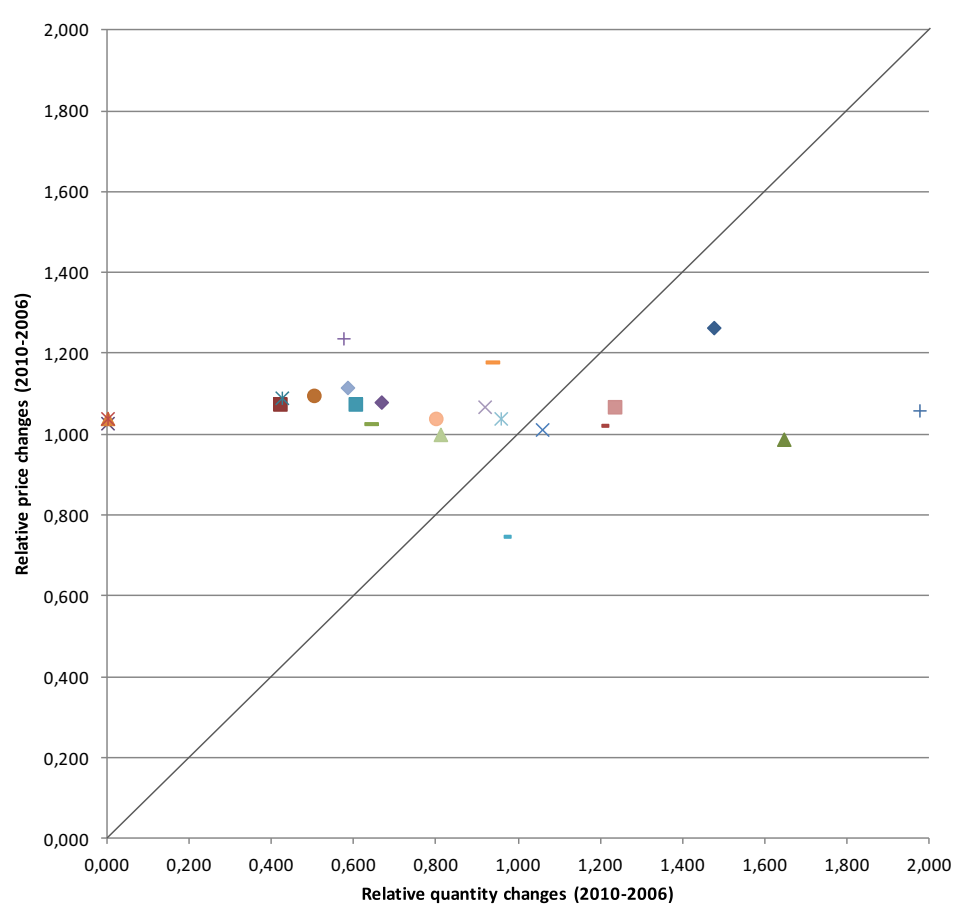

-022 Wood in the rough

161 Wood, sawn and planed

$\triangle 1621$ Veneer sheets and wood-based panels

$\times 1623$ Other builders carpentry and joinery

* 1629 Other products of wood; articles of cork, straw and

plaiting materials

- 171 Pulp, paper and paperboard

-20 Chemicals and chemical products

- 23 Other non-metallic mineral products

- 900 Other material and commodity input

$\triangle 921$ Operating materials (without packaging)

$\times 922$ Packaging materials, e.g. wood, paper, cardboard

* 923 Supplies for company kitchens and canteens

+ 932 Liquid fuels

933 Gas, e.g. natural gas or liquefied petroleum

-934 Electricity

935 District heating (steam) and compressed air

Costs for hired labour

$\triangle$ Costs for other industrial and mechanical services

$\times$ Costs for temporary workforces

$\times$ Rents and leases

Other costs (total)

Fig. 7. Relative changes in price and quantity of the intermediate input of ES 1621 "Veneer sheets and wood-based panels" for the year 2010 compared to 2006.

volume: 3.857), 935 "district heating (steam) and compressed air" (growth factor of price: 1.226, growth factor of volume: 2.233) were deleted as outliers. However, in 2006 the relative share of these intermediate input positions of the gross production value for 1629 "other products of wood; articles of cork, straw and plaiting materials" was only $0.6 \%$ and for 935 "district heating (steam) and compressed air" only $0.5 \%$.

Fig. 7 shows the growth factor of prices and quantities for selected input items of ES 1621 for the year 2010 compared to 2006. In this comparison a relative decrease of quantity of almost all intermediate input positions, mirrored in the decrease of absolute intermediate input of $4.14 \mathrm{~B} €$ in 2006 and $4.06 \mathrm{~B} €$ in 2010 becomes apparent. However the growth factor of quantity of 022 "wood in the rough" increased (1.476\%) whereas 161 "wood, sawn and planed" decreased (0.42). As in ES 1610 the growth factor of price of the product groups 022 "wood in the rough" and 161 "wood, sawn and planed" was 1.264 and 1.075. The weighted average growth factor of prices and quantities of the total intermediate input of ES 1621 was 1.061 and 0.921 . In Fig. 7 only 931 "solid fuels", with a growth factor of price of 1.10 and a growth factor of volume of 2.243 , was deleted as an outlier. The share of the 931 "solid fuels" of the gross production value was $0.4 \%$ in 2010 .

In summary, apart from some outliers, we determined a quite heterogenic development of the growth factors of prices and quantities of the individual intermediate input positions. However, comparing the year 2010 to 2006 a decrease of the growth factors of quantities becomes visible for ES 1610 as well as for ES 1621.

\subsection{Effects of changes in value added}

As is visualised in Fig. 1 the signs for the I) product price effect and III.a) growth effect (output quantity) are positive, the signs of the II) intermediate input price effect, the (III.b) growth effect (input quantity) and the IV) structural effect are negative. The input data, derived from the cost structure statistics, the price statistics and material and commodity input statistics, for the calculation of the four elements of change in value added of ES 1610 and ES 1621, as shown in Tables 1 and 2, are given in Appendix E.

Comparing the year 2006 to 2002 the changes in value added of $240,345 \mathrm{~T} €$ in sum were composed of the product price effect with 601,126 thousand $€(T €)$, the intermediate input price effect with $-351,243 \mathrm{~T} €$, the growth effect with 207,980 T€ and the structural effect with $-217,518 \mathrm{~T} €$ in ES 1610. Thus, the change in value added was mainly driven by the product price effect which was stronger than both, intermediate input price effect and growth effect. The structural effect and the growth effect are largely balanced out indicating, that while there was a growth effect, outsourcing and offshoring happened in almost equal manner. In a further step, the growth effect can be split in the growth effect of the output with 862,534 T€ and the growth effect of the intermediate input with $-654,554 \mathrm{~T} €$. In this case, the growth effect of the output was stronger than that of the intermediate input. This means that the increase in output quantity was higher than the increase of input quantity.

Looking at the year 2010 the picture is quite different. On first glance it becomes apparent, that the change in gross value added is negative $(-202,034 \mathrm{~T} €)$, i.e., the gross value added in 2010 decreased compared to 2006. The product price effect is $311,028 \mathrm{~T} €$ and thus declined to $52 \%$ of the comparison of the year 2006 to 2002. The intermediate input

Table 1

Effects of change of gross value added of ES 1610 "Sawmilling and planing of wood".

\begin{tabular}{lcc}
\hline ES 1610 & $\begin{array}{l}\text { 2006 compared } \\
\text { to 2002 (in T€) }\end{array}$ & $\begin{array}{c}\text { 2010 compared } \\
\text { to 2006 (in T€) }\end{array}$ \\
\hline I. Product price effect & 601,126 & 311,028 \\
II. Intermediate input price effect & $-351,243$ & $-467,679$ \\
III. Growth effect & 207,980 & $-158,909$ \\
$\quad$ III.a Growth effect (output quantity) & 862,534 & $-729,050$ \\
III.b Growth effect (input quantity) & $-654,554$ & 570,141 \\
IV. Structural effect & $-217,518$ & 113,526 \\
Sum (=Change of gross value added) & 240,345 & $-202,034$ \\
\hline
\end{tabular}


Table 2

Effects of change of gross value added of ES 1621 "Manufacture of veneer sheets and wood-based panels".

\begin{tabular}{lcc}
\hline ES 1621 & $\begin{array}{l}\text { 2006 compared } \\
\text { to 2002 (in T€) }\end{array}$ & $\begin{array}{c}\text { 2010 compared } \\
\text { to 2006 (in T€) }\end{array}$ \\
\hline I. Product price effect & 642,434 & 340,393 \\
II. Intermediate input price effect & $-404,511$ & $-234,957$ \\
III. Growth effect & 46,385 & $-136,289$ \\
III.a Growth effect (output quantity) & 168,891 & $-672,978$ \\
III.b Growth effect (input quantity) & $-122,507$ & 536,688 \\
IV. Structural effect & $-436,196$ & $-216,678$ \\
Sum (=Change of gross value added) & $-151,889$ & $-247,532$ \\
\hline
\end{tabular}

price effect with $-467,679 \mathrm{~T} €$ is $133 \%$ higher than in 2006 compared to 2002 and stronger than the product price effect: more has been spent on intermediate input than could be compensated by output prices. Also both, growth effect of the intermediate input quantity and the growth effect of the output quantity strongly declined indicating a poor demand situation on the market. The growth effect in 2010 compared to 2006 is with $-158,909 \mathrm{~T} €$ negative, the structural effect, in contrast to 2006, is with 113,034 T€ positive which suggests a decline of growth and of outsourcing activity. The change in value added is driven mainly by high amounts of input.

With $-151,899 \mathrm{~T} €$ the change in value added of the economic sector ES 1621 was negative in the year 2006 compared to 2002 (Table 2). In the year 2006 the product price effect was $642,434 \mathrm{~T} €$, the intermediate input effect $-404,511 \mathrm{~T} €$, the growth effect $46,385 \mathrm{~T} €$ and the structural effect $-436,196 \mathrm{~T} €$. As in the economic sector ES 1610, the product price effect in this the year 2006 compared to 2002 was stronger than both, intermediate input price effect and growth effect. Also, in the year 2006, the structural effect was very strong, indicating high outsourcing activity. The growth effect, with $46,385 \mathrm{~T} €$ is only a little more than a quarter of, for example, the growth effect in the same year of the economic sector ES 1610 with 207,980 T€.

In the year 2010 compared to 2006 , with $-247,532 \mathrm{~T} €$, there is also a negative change of value added. Both, product price effect $(340,393 \mathrm{~T}$ $€)$ and intermediate input price effect $(-234,957 \mathrm{~T} €$ ) declined to $53 \%$ and $58 \%$, respectively, compared to the values of 2006 to 2002 . Howev$\mathrm{er}$, the product price effect still exceeds the intermediate input price effect. Also both, growth effect of input and output quantity sharply declined. The growth effect of the output quantity with $-672,978 \mathrm{~T} €$ and the growth effect of the input quantity with 536,688 T€ experienced the strongest decreases and lead to a negative overall growth effect $(-136,289 \mathrm{~T} €)$. The structural effect with $-216,678 \mathrm{~T} €$ sank by $50 \%$ compared to the year 2006 to 2002.

\section{Discussion and conclusion}

In this paper we aimed to introduce an approach to decompose the effects of changes of value added and to apply it on ES 1610 and ES 1621 as two case study objects. By means of the decomposition analysis the effects triggering changes in value added were demonstrated. Furthermore, a special focus was put on the role of the intermediate input of wood and wood-based products within the gross production value.

\subsection{Discussion of methods}

Our developed approach of a decomposing analysis attributes changes of value added to the four effects I) changes in (nominal) product prices, II) changes in (nominal) prices of input goods for intermediate consumption, III) changes in output of products and IV) changes in shares of intermediate input and value added due to outsourcing and offshoring. All in all the approach provides feasible results and an explanatory contribution for changes in value added. However, we are fully conscious that in economic practices changes in value added are caused by a multitude of factors such as market conditions, consumer trends, seasonal effects, national and international competition as well as government regulations.

In this study our approach was developed according to the PaascheIndex (Frenkel and John, 1999: $118 \mathrm{f}$ ). One of the disadvantages of the Paasche-Index in a timeline is its ever-changing consumer basket meaning that the result is influenced by quantity changes. Substitutional effects are not considered. Hence, comparability between years in a timeline can be hindered. The Laspeyres-Index, in contrast, would be easier to handle, as the basket of commodities with its quantities and qualities does not change every year. However, within the PaascheIndex today's prices and quantities are weighted stronger than past prices and quantities. In our study we chose to evaluate changes in value added for single years in a backwards perspective. The actuality of data used to for the Paasche-Index was thus deemed an advantage for our approach. The focus on price as well as quantity effects was a desired and essential part in our study.

The results of our approach of decomposition are, as most economic models, dependent on data availability and data quality. In order to determine changes in value added of ES 1610 and ES 1621 we used official statistics from the German Federal Statistical Office, especially the cost structure statistics. Within the intermediate input groups of the cost structure statistics for ES 1610 and ES 1621 the group "consumption of raw materials and operating supplies" has a share of about $50-70 \%$ of the gross production value. Yet, this group is not further differentiated within the statistics. In order to enhance the input data for the decomposition analysis, by calculating a weighted average of price and quantity changes over all intermediate input positions, the material and commodity input statistics was integrated into the cost structure statistics. This is possible as both statistics feature equal statistical reporting categories and identical registration units, enabling a more precise and differentiated determination of the input. As positive side effects and for an explanatory contribution, this approach enabled us to determine the significance of wood-based commodity groups as input factors as well as the differentiated consideration of price and quantity changes of all intermediate input positions.

The origination of the gross production value is only roughly differentiated within the cost structure statistics. Next to the gross production value the total turnover as well as the turnover from 1.) in-house products and industrial service, 2.) trade goods and commission trade and 3.) other activities is designated. This classification is rather rough and does, hence, not allow for a linkage to the respective product indices of the price statistics. Thus, whereas the weighted price changes of the intermediate input could be determined, a blanket approach with a generalised price index of the StBA for the output had to be used. The use of differentiated data for calculating output changes within the decomposition analysis would be highly desirable. A possible approach to this problem could be the use of the production survey (StBA F4 R3.1). However, registration units of the production survey are clearly dissenting compared to the cost structure statistics and the material and commodity input statistics. Where the cost structure statistics and the material and commodity input statistics contain data of enterprises classified by their main activity and the "Classification of Material and Goods Received in Manufacturing, Mining and Quarrying" (WE2002 and WE2010) the production survey functionally features the entire production of enterprises of manufacturing, mining and quarrying.

Due to the quadrennial appearance of the material and commodity input statistics a continuous time line analysis could not be carried out. Due to this data availability the years 2002, 2006 and 2010 were considered for our evaluation. A transfer of the share of intermediate input positions to the cost structure statistics of in-between years was, however, not deemed to be plausible. Comparing the years 2010 and 2006 no significant distortions must be expected by the restructuring 
of the "Product Classification for Production Statistics" (GP2002 to GP2009). In regard to the restructuring of the "Classification of Material and Goods Received in Manufacturing, Mining and Quarrying" (WE 2003 to WE 2008) the assignment of pellets and other woody fuels from the 1621 "wood sawn and planed" to 1629 "other products of wood; articles of cork, straw and plaiting materials" must be kept in mind.

\subsection{Discussion of results}

For an examination of changes of input, output and value added the economic sectors ES 1610 and ES 1621, both major players in the wood-based sector, were chosen in this study. The results of the decomposition analysis enable an appraisal of the changes in value added for the economic sectors.

The extent of influence of the four different effects on changes in value added differed in the two years considered. Comparing 2006 to 2002, ES 1610 experienced an increase of gross value added. This change was mainly driven by the product price effect which surpassed the intermediate input effect by $171 \%$. Revenue from output over-compensated the costs for input. Another strong factor in 2006 was the output quantity which exceeded the input quantity by $132 \%$. This indicates an increase in efficiency, resulting in a higher volume of output referred to a given volume of input. The results mirror the development of the enterprises of the economic sector ES 1610. Benefitting - as the timber market in general - from ready foreign markets, the turnover of the wood-based industries and the sales quantities of sawn timber, wood-based panels and other semi-finished wood products could be increased (Dieter, 2005, 2006, 2007; Seintsch, 2010, 2011a; Seintsch and Weimar, 2013). Furthermore the strong product price effect was further enhanced by the high proceeds for sawmill by-products, such as sawdust, that could be attained caused by the rising demand for wood for energetic uses (Mantau, 2009). Also, increases in international competitiveness (Dieter and Englert, 2007, 2009) and in turnover of foreign trade were achieved (StBA F7 R2; StBA F4 R4.1.1). Against the background of these favourable conditions an expansion of capacities within the first timber processing stage took place, presumably favouring the increase of efficiency in production and thus the increase of output quantity. Gains through economies of scale could have followed and could also have improved the growth effect. At the same time, however, the domestic potentials of coniferous timber declined due to domestic demand (Ochs et al., 2007) Also, since 2005, an increase of timber prices was noted (Ermisch et al., 2013; StBA F17 R 2). These developments indicate a change from a supply to a demand timber market. This in turn backs our analysis of the single intermediate input positions, which showed a relative quantity increase of wood and wood-based products in 2006 compared to 2002. The growth effect and the structural effect, in contrast, were roughly balanced, indicating outsourcing activities but also growth within the enterprises. In spite of the abovementioned developments, and although the product price effect exceeded the intermediate input price effect, ES 1621, in turn, experienced a negative change of gross value added. As in ES 1610 this was driven mainly by high expenses for intermediate input of almost two thirds of the product price effect and also by the strong structural effect of $-436,169 \mathrm{~T} €$. This might be explained by high demands on ready (foreign) markets and necessary outsourcing and offshoring due to order situations. The expenses for woodbased input prices can be explained by the ever-growing domestic uses of timber, likewise in the energetic sector, as well as by the expansion of capacities in the wood processing industry. These developments lead to competing demands of the resource wood and caused an increase of prices for formerly low priced raw materials like wood of inferior quality or sawmilling by-products for ES 1621 (Ochs et al., 2007; Mantau, 2009). As can also be seen in Appendix
D.1 and D.2, ES 1621 noted equally high changes of relative input prices than ES 1610 (110.8\% in ES 1621 compared to 110.2\% in ES 1610 ) but lower relative input quantities (116.4\% in ES 1621 compared to $135 \%$ in ES 1610).

Comparing the year 2010 to 2006 there was a negative gross value added in both, ES 1610 and ES 1621. We assume that this was mainly due to poor order situations and the reduction of overall production capacity in the first timber processing stage (Seintsch, 2011b). These were mainly caused by the financial crisis which started in the summer of 2007 and receded in 2009. The crisis showed how strong the German ES 1610 and ES 1621 had become in the global market, profiting from the real estate boom in the USA and Europe and suffering from the sudden lack of demand when that market crashed (Mantau, 2009). The years between 2006 and 2010 were also characterised by a further domestic demand of timber, especially for energetic uses (Mantau, 2009, 2012) and a further increase of timber prices (StBA F17 R 1; Ermisch et al., 2013). In ES 1610 these developments are mirrored by an intermediate input price effect which exceeded the product price effect: Due to the decline of demand, the higher amount spent on intermediate input could not be compensated by output revenues. In ES 1621 the product price effect still exceeded the intermediate input price effect but was reduced by half as compared to 2006. In both economic sectors, the reduction of product and intermediate input price effect could be attributed to the decline of demand due to after-effects of the economic crisis. Capacity adjustments or lack of investments due to the poor demand situations are also likely. The negative gross value added of both economic sectors was also influenced by the strongly declined, or even negative, output and input quantity which caused a negative growth effect for both sectors and, in the case of ES 1610 , a negative structural effect. The decreasing demand on the world market, which in turn induces a lesser need for outsourced production to complete orders, could again be attributed to this development.

The structural effect can in general be regarded as an indicator for a decrease in outsourcing and offshoring activities. Wood-based intermediate input, especially the product groups that are the main output of the considered economic sector, could in turn be regarded as products of outsourcing and offshoring, especially, when enterprises specialise in trading activities. For both sectors the decline of offshoring and outsourcing in the year 2010 is reflected in the general decline of quantity of intermediate input (Figs. 5 and 7). In ES 1610 it is also mirrored in the decline of input quantity of wood-based products: The growth factor in 2010 compared to 2006 of 161 "wood, sawn and planed" was 0.611 , of 1621 "veneer sheets and wood-based panels" 0.352 and of 1623 "other builders carpentry and joinery" 0.162 . In ES 1621 , in contrast, the intermediate input product 1621 "veneer sheets and wood-based panels", which is the also the main output of the branch, was increased in quantity in 2010 (growth factor: 1.646) whereas 161 "wood, sawn and planed" decreased (growth factor: $0.42){ }^{2}$ In this economic sector almost all of the other intermediate input declined, amongst them 20 "chemicals and chemical products" (growth factor: 0.642 ), presumably containing adhesives needed for the production of the wood-based panels.

The changes of value added are subject to changes of price and quantity of the intermediate input and output. Changes of price and quantity of the total intermediate input are, in turn, influenced by the single positions of the intermediate input. As could be shown in our study, raw wood and wood-based products play an important role in the intermediate input mix of ES 1610 and ES 1621. In ES 1610 raw wood and wood-based products had a share of the gross production value of $54 \%$

\footnotetext{
2 For 1623 “other builders' carpentry and joinery”, a value of intermediate input was not available for 2010 .
} 
in 2002 and 2006 and 60\% in 2010. The share of raw wood of the gross production value increased from $34.6 \%$ in 2002 to $41.5 \%$ in 2010 . With shares of the gross production value of $25 \%$ in $2002,28 \%$ in 2006 and $39 \%$ in 2010 the status of raw wood and wood-based products in ES 1621 is not as high in ES 1610 but plays an important role still. The share of raw wood of the gross production value in ES 1621 also increased from $7.5 \%$ in 2002 to $14.0 \%$ in 2010 . The input of veneer sheets and wood-based panels, in contrast, almost doubled from $12.5 \%$ in 2002 up to $21.4 \%$ in 2010, presumably as a substitution for 161 "wood, sawn and planed" which had increased in price between 2006 and 2010. Figs. 4-7 show the relative changes in price and quantity of the single intermediate input positions of ES 1610 and ES 1621. As can be seen, changes in the main positions 022 "wood in the rough", 161 "wood, sawn and planed" and 1621 "veneer sheets and wood-based panels" follow the general trends of the changes of intermediate input: In 2006 changes in expenses for intermediate input were mostly due to quantity changes and in 2010 due to price changes. In general, the correlation for the total intermediate input seems also to apply for wood and wood-based products.

The only exceptions are 022 "wood in the rough" and 1621 "veneer sheets and wood-based panels" in ES 1621 in the year 2010. This might be due to scarcity of raw materials and substitution with other woodbased products or to reductions of stock.

In general, a proportional development of the wood input as compared to the growth factor is expected. With our results the close connection between the growth effect and the wood input of ES 1610 is visualised. The growth effect in 2006 had a share of $25.4 \%$ as compared to the gross value added of 2002. In 2010 this share was - 15\% of the gross value added of 2006. Assuming the same (and constant) prices for all wood-based input goods the amount of input changed on average by $+37 \%$ in 2006 compared to 2002 and by $-13 \%$ in 2010 compared to 2006. Thus it becomes visible, that wood input follows the same development as the growth factors and is in the same range in 2010 compared to 2006.

The situation for ES 1621 is a different one: The growth effect of ES 1621 had a share of $3.9 \%$ of the gross value added of 2002 and in 2010 of $-13 \%$ of the gross value added of 2006 . Assuming the same (and constant) prices for all wood-based input goods the amount of input changed by $+12 \%$ in 2006 compared to 2002 and again by $+35 \%$ in 2010 compared to 2006. Thus, in 2006 to 2002 a positive development in both growth effect and wood input is given. However, in 2010 compared to 2006 there was an opposing development: more wood-based products were applied then before while but at the same time there was a negative growth effect.

On the one hand, this could be due to statistical errors. When regarding the wood input given in the Arbeitsunterlage Rohholz and the output of wood based panels of the StBA of the past years an implausible relation becomes visible: there is more wood input than produced panels (Weimar, 2011). On the other hand we assume that wood purchased for energetic uses is also entered in the receipt of goods for production and might thus falsify the material and commodity input statistics.

In conclusion outsourcing and offshoring do, compared to the other effects, play a minor role only in the two economic sectors. A higher input of raw wood or wood based products does not necessarily lead to a higher value added in ES 1610 and ES 1621 in the years considered. Whereas the share of raw wood and wood-based products as intermediate input of the gross production value in both economic sectors increased, the value added strongly decreased between 2002 and 2006 for ES 1621 and between 2006 and 2010 for ES 1610, respectively. The growth effect, though, is connected to the wood input in ES 1610: the increase or decrease of the growth effect is proportional to the wood input of this sector. This is not the case for ES 1621 which might be due to accounting practices.

However, explanatory approaches, linking wood input to value added in the wood-based industry are too short-sighted. Other effects like domestic or foreign outsourcing or developments on the world market, influencing timber prices, play a major role as well.

\section{Acknowledgements}

The authors wish to express their gratitude to the German Federal Ministry of Education and Research (BMBF) for funding the joint research project "Bioenergie-Regionen stärken (BEST, grant number 033L033C)" within the scope of which the research for this publication was conducted. The sole responsibility for the contents of this publication rests with the authors.

The authors would also like to thank Mr. Hermann Englert (Thünen Institute of International Forestry and Forest Economics, Hamburg, Germany) cordially for his substantial support, especially with the geometrical proof recorded in Appendix A.2. Last but not least we thank the anonymous reviewers for their comments and suggestions for the enhancements of this paper.

\section{Appendix A}

A.1

$$
\begin{aligned}
\Delta V A= & X_{1}^{G} * P_{1}^{G}-X_{1}^{G} * P_{0}^{G} \\
& +X_{1}^{V} * P_{0}^{V}-X_{1}^{V} * P_{1}^{V} \\
& +\left(X_{1}^{G} * P_{0}^{G}-X_{0}^{G} * P_{0}^{G}\right) *\left(1-S_{0}^{V}\right) \\
& +S_{0}^{V} * X_{1}^{\mathrm{V}} * P_{0}^{G}-X_{1}^{V} * P_{0}^{V} .
\end{aligned}
$$

With

$\mathrm{S}_{0}^{\mathrm{V}}=\frac{\mathrm{X}_{0}^{\mathrm{V}} \times \mathrm{P}_{0}^{\mathrm{V}}}{\mathrm{X}_{0}^{\mathrm{G}} \times \mathrm{P}_{0}^{\mathrm{G}}}$

$$
<=>
$$

$$
\begin{aligned}
& \Delta \mathrm{VA}=\mathrm{X}_{1}^{\mathrm{G}} * \mathrm{P}_{1}^{\mathrm{G}}-\mathrm{X}_{1}^{\mathrm{G}} * \mathrm{P}_{0}^{\mathrm{G}}+ \\
& \begin{array}{l}
+\mathrm{X}_{1}^{\mathrm{V}} * \mathrm{P}_{0}^{\mathrm{V}}-\mathrm{X}_{1}^{\mathrm{V}} * \mathrm{P}_{1}^{\mathrm{V}}+ \\
+\left(\mathrm{X}_{1}^{\mathrm{G}} * \mathrm{P}_{0}^{\mathrm{G}}-\mathrm{X}_{0}^{\mathrm{G}} * \mathrm{P}_{0}^{\mathrm{G}}\right) *\left(1-\frac{\mathrm{X}_{0}^{\mathrm{V}} * \mathrm{P}_{0}^{\mathrm{V}}}{\mathrm{X}_{0}^{\mathrm{G}} * \mathrm{P}_{0}^{\mathrm{G}}}\right)+
\end{array} \\
& +\frac{\mathrm{X}_{0}^{\mathrm{V}} * \mathrm{P}_{0}^{\mathrm{V}}}{\mathrm{X}_{0}^{\mathrm{G}} * \mathrm{P}_{0}^{\mathrm{G}}} * \mathrm{X}_{1}^{\mathrm{G}} * \mathrm{P}_{0}^{\mathrm{G}}-\mathrm{X}_{1}^{\mathrm{V}} * \mathrm{P}_{0}^{\mathrm{V}} \text {. } \\
& \Delta \mathrm{VA}=\mathrm{X}_{1}^{\mathrm{G}} * \mathrm{P}_{1}^{\mathrm{G}}-\mathrm{X}_{1}^{\mathrm{G}} * \mathrm{P}_{0}^{\mathrm{G}}+ \\
& +\mathrm{X}_{1}^{\mathrm{V}} * \mathrm{P}_{0}^{\mathrm{V}}-\mathrm{X}_{1}^{\mathrm{V}} * \mathrm{P}_{1}^{\mathrm{V}}+ \\
& +\mathrm{X}_{1}^{\mathrm{G}} * \mathrm{P}_{0}^{\mathrm{G}}-\mathrm{X}_{0}^{\mathrm{G}} * \mathrm{P}_{0}^{\mathrm{G}}-\mathrm{X}_{1}^{\mathrm{G}} * \mathrm{P}_{0}^{\mathrm{G}} * \frac{\mathrm{X}_{0}^{\mathrm{V}} * \mathrm{P}_{0}^{\mathrm{V}}}{\mathrm{X}_{0}^{\mathrm{G}} * \mathrm{P}_{0}^{\mathrm{G}}}+\mathrm{X}_{0}^{\mathrm{G}} * \mathrm{P}_{0}^{\mathrm{G}} * \frac{\mathrm{X}_{0}^{\mathrm{V}} * \mathrm{P}_{0}^{\mathrm{V}}}{\mathrm{X}_{0}^{\mathrm{G}} * \mathrm{P}_{0}^{\mathrm{G}}}+ \\
& +\frac{\mathrm{X}_{0}^{\mathrm{V}} \times \mathrm{P}_{0}^{\mathrm{V}}}{\mathrm{X}_{0}^{\mathrm{G}} \times \mathrm{P}_{0}^{\mathrm{G}}} * \mathrm{X}_{1}^{\mathrm{G}} * \mathrm{P}_{0}^{\mathrm{G}}-\mathrm{X}_{1}^{\mathrm{V}} * \mathrm{P}_{0}^{\mathrm{V}} \\
& <=> \\
& \Delta \mathrm{VA}=\mathrm{X}_{1}^{\mathrm{G}} * \mathrm{P}_{1}^{\mathrm{G}}-\mathrm{X}_{1}^{\mathrm{G}} * \mathrm{P}_{0}^{\mathrm{G}}+ \\
& +\mathrm{X}_{1}^{\mathrm{V}} * \mathrm{P}_{0}^{\mathrm{V}}-\mathrm{X}_{1}^{\mathrm{V}} * \mathrm{P}_{1}^{\mathrm{V}}+ \\
& +\mathrm{X}_{1}^{\mathrm{G}} * \mathrm{P}_{0}^{\mathrm{G}}-\mathrm{X}_{0}^{\mathrm{G}} * \mathrm{P}_{0}^{\mathrm{G}}-\mathrm{X}_{1}^{\mathrm{G}} * \frac{\mathrm{X}_{0}^{\mathrm{V}} * \mathrm{P}_{0}^{\mathrm{V}}}{\mathrm{X}_{0}^{\mathrm{G}}}+\mathrm{X}_{0}^{\mathrm{V}} * \mathrm{P}_{0}^{\mathrm{V}}+ \\
& +\frac{\mathrm{X}_{0}^{\mathrm{V}} * \mathrm{P}_{0}^{\mathrm{V}}}{\mathrm{X}_{0}^{\mathrm{G}}} * \mathrm{X}_{1}^{\mathrm{G}}-\mathrm{X}_{1}^{\mathrm{V}} * \mathrm{P}_{0}^{\mathrm{V}} \text {. } \\
& <=> \\
& \Delta \mathrm{VA}=\mathrm{X}_{1}^{\mathrm{G}} * \mathrm{P}_{1}^{\mathrm{G}}-\mathrm{X}_{1}^{\mathrm{V}} * \mathrm{P}_{1}^{\mathrm{V}}-\mathrm{X}_{0}^{\mathrm{G}} * \mathrm{P}_{0}^{\mathrm{G}}+\mathrm{X}_{0}^{\mathrm{V}} * \mathrm{P}_{0}^{\mathrm{V}} . \\
& <=> \\
& \Delta V A=\left(X_{1}^{G} * P_{1}^{G}-X_{1}^{V} * P_{1}^{V}\right)-\left(X_{0}^{G} * P_{0}^{G}-X_{0}^{V} * P_{0}^{V}\right)
\end{aligned}
$$




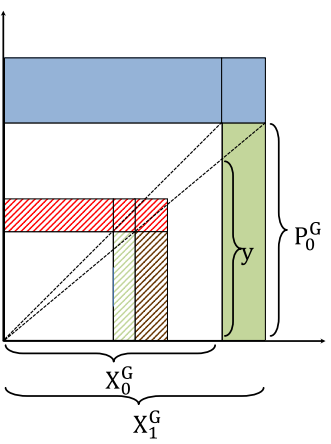

$\frac{P_{0}^{G}}{y}=\frac{X_{1}^{G}}{X_{0}^{G}}$

for $X_{0}^{G}=1$ and $P_{0}^{G}=1$ it follows

$y=\frac{1}{X_{1}^{G}}$

Fig. A1. For specification see Fig. 1 in the text.

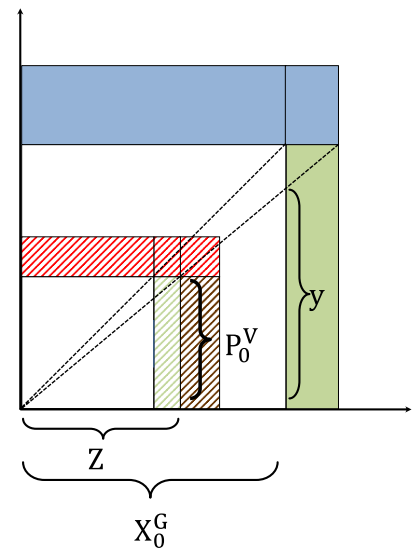

$\frac{y}{P_{0}^{V}}=\frac{X_{0}^{G}}{Z}$

for $X_{0}^{G}=1$ it follows

$Z=\frac{P_{0}^{V}}{y}$

Fig. A2. For specification see Fig. 1 in the text.
$\mathrm{Z}=\frac{\mathrm{P}_{0}^{\mathrm{V}}}{\mathrm{y}}=\mathrm{P}_{0}^{\mathrm{V}} * \mathrm{X}_{1}^{\mathrm{G}}$

Alternative proof from Figs. A1 and A2:

$\frac{P_{0}^{G}}{X_{1}^{G}}=\frac{P_{0}^{V}}{Z} ;$ for $P_{0}^{G}=1$ it follows $Z=P_{0}^{V} * X_{1}^{G}$.

Area of the rectangle in question:

$\mathrm{P}_{0}^{\mathrm{V}} * \mathrm{X}_{1}^{\mathrm{G}} * \mathrm{P}_{0}^{\mathrm{V}}-\mathrm{X}_{0}^{\mathrm{V}} \mathrm{P}_{0}^{\mathrm{V}}=\mathrm{P}_{0}^{\mathrm{V}}\left(\mathrm{X}_{1}^{\mathrm{G}} * \mathrm{P}_{0}^{\mathrm{V}}-\mathrm{X}_{0}^{\mathrm{V}}\right)$

Accordance with term III.a in Eq. (1)

$\frac{\mathrm{X}_{0}^{\mathrm{V}} * \mathrm{P}_{0}^{\mathrm{V}}}{\mathrm{X}_{0}^{\mathrm{G}} * \mathrm{P}_{0}^{\mathrm{G}}}\left[\left(\mathrm{X}_{1}^{\mathrm{G}}-\mathrm{X}_{0}^{\mathrm{G}}\right) * \mathrm{P}_{0}^{\mathrm{G}}\right]$; for $\mathrm{X}_{0}^{\mathrm{G}}=1$ and $\mathrm{P}_{0}^{\mathrm{G}}=1$ it follows

$\mathrm{X}_{0}^{V} * \mathrm{P}_{0}^{\mathrm{V}}\left[\left(\mathrm{X}_{1}^{\mathrm{G}}-1\right)\right]$

$=\mathrm{P}_{0}^{\mathrm{V}}\left(\mathrm{X}_{1}^{\mathrm{G}} * \mathrm{X}_{0}^{\mathrm{V}}-\mathrm{X}_{0}^{\mathrm{V}}\right)$.

Eq. (3) = Eq. (4) only if

$\mathrm{P}_{0}^{V} *\left(\mathrm{X}_{1}^{\mathrm{G}} * \mathrm{P}_{0}^{\mathrm{V}}-\mathrm{X}_{0}^{\mathrm{V}}\right)=\mathrm{P}_{0}^{\mathrm{V}}\left(\mathrm{X}_{1}^{\mathrm{G}} * \mathrm{X}_{0}^{\mathrm{V}}-\mathrm{X}_{0}^{\mathrm{V}}\right) \quad<=>\quad \mathrm{P}_{0}^{\mathrm{V}}=\mathrm{X}_{0}^{\mathrm{V}}$.

\section{Appendix B. Cost structure statistics}

\begin{tabular}{|c|c|c|c|c|c|c|}
\hline \multirow[t]{2}{*}{ Cost structure statistics (StBA F4 R 4.3) } & \multicolumn{3}{|c|}{ ES 1610 “Sawmilling and planing of wood” (T€) } & \multicolumn{3}{|c|}{ ES 1621 “Veneer sheets and wood-based panels” (T€) } \\
\hline & 2002 & 2006 & 2010 & 2002 & 2006 & 2010 \\
\hline Receipt of raw materials and operating supplies (incl. energy) & $1,909,478$ & $2,832,418$ & $2,682,070$ & $2,244,895$ & $2,977,526$ & $2,722,707$ \\
\hline Initial stocks less final stocks of raw materials and operating supplies & 23,349 & $-43,752$ & $-50,364$ & 30,467 & 768 & $-13,673$ \\
\hline \multicolumn{7}{|l|}{ Consumption of raw materials and operating supplies (material) } \\
\hline Consumption of raw materials and operating supplies (total) & $1,932,827$ & $2,788,666$ & $2,631,706$ & $2,275,362$ & $2,978,294$ & $2,709,035$ \\
\hline Of which energy & 67,828 & 115,156 & 167,069 & 194,692 & 315,825 & 307,399 \\
\hline Receipt of commodities & 193,064 & 288,415 & 371,735 & 151,365 & 174,792 & 554,034 \\
\hline Initial stocks less final stocks of commodities & -5477 & -4500 & $-11,644$ & -1093 & -2396 & 2349 \\
\hline Consumption of commodities & 187,587 & 283,915 & 360,091 & 150,271 & 172,396 & 556,383 \\
\hline Costs for hired labour & 36,365 & 46,382 & 49,111 & 23,632 & 31,663 & 41,759 \\
\hline \multicolumn{7}{|l|}{ Other intermediate input } \\
\hline Costs for other industrial and mechanical services & 86,126 & 117,509 & 89,612 & 132,562 & 146,764 & 118,941 \\
\hline Costs for temporary workforces & 5112 & 24,659 & 23,345 & 18,829 & 26,973 & 26,458 \\
\hline Rents and leases & 52,736 & 81,232 & 78,294 & 85,903 & 97,533 & 97,058 \\
\hline Other costs (total) & 277,612 & 459,318 & 353,535 & 493,419 & 689,570 & 572,827 \\
\hline Taxes other than on income (total) & 16,468 & 39,736 & 21,543 & 15,292 & 19,901 & 10,357 \\
\hline Interest expenses & 59,475 & 57,067 & 71,030 & 86,860 & 74,971 & 62,348 \\
\hline
\end{tabular}

\section{Appendix C. Data bases for price indices}

\begin{tabular}{|c|c|c|c|c|c|}
\hline \multicolumn{3}{|c|}{ Intermediate input (absolute) } & \multicolumn{3}{|l|}{ Price indices } \\
\hline Abbr. & Identification & Data base & Abbr. & Identification & Data base \\
\hline 022 & Wood in the rough & StBA F4 R4.2.4 & GP09-022 & Wood in the rough & Price index for industrial products \\
\hline 161 & Wood, sawn and planed & StBA F4 R4.2.4 & GP09-161 & Wood, sawn and planed & Price index for industrial products \\
\hline 162 & Veneer sheets and wood-based panels & StBA F4 R4.2.4 & GP09-1621 & Veneer sheets and wood-based panels & Price index for industrial products \\
\hline 31 & Furniture & StBA F4 R4.2.4 & GP09-31 & Furniture & Price index for industrial products \\
\hline
\end{tabular}


Appendix C (continued)

\begin{tabular}{|c|c|c|c|c|c|}
\hline \multicolumn{3}{|c|}{ Intermediate input (absolute) } & \multicolumn{3}{|l|}{ Price indices } \\
\hline Abbr. & Identification & Data base & Abbr. & Identification & Data base \\
\hline 25 & $\begin{array}{l}\text { Fabricated metal products, except } \\
\text { machinery and equipment }\end{array}$ & StBA F4 R4.2.4 & GP09-25 & $\begin{array}{l}\text { Fabricated metal products, except } \\
\text { machinery and equipment }\end{array}$ & Price index for industrial products \\
\hline 13 & Textiles & StBA F4 R4.2.4 & GP09-13 & Textiles & Price index for industrial products \\
\hline 222 & Plastic products & StBA F4 R4.2.4 & GP09-222 & Plastic products & Price index for industrial products \\
\hline 171 & Pulp, paper and paperboard & StBA F4 R4.2.4 & GP09-171 & Pulp, paper and paperboard & Price index for industrial products \\
\hline 20 & Chemicals and chemical products & StBA F4 R4.2.4 & GP09-20 & Chemicals and chemical products & Price index for industrial products \\
\hline 23 & Other non-metallic mineral products & StBA F4 R4.2.4 & GP09-23 & Other non-metallic mineral products & Price index for industrial products \\
\hline 24 & Basic metals & StBA F4 R4.2.4 & GP09-24 & Basic metals & Price index for industrial products \\
\hline 27 & Electrical equipment & StBA F4 R4.2.4 & GP09-27 & Electrical equipment & Price index for industrial products \\
\hline 921 & Operating materials (without packaging) & StBA F4 R4.2.4 & & & Average German producer price index \\
\hline 922 & Packaging materials, e.g., wood, paper, cardboard & StBA F4 R4.2.4 & GP09-1624 & $\begin{array}{l}\text { Packaging materials, e.g., wood, } \\
\text { paper, } \\
\text { cardboard }\end{array}$ & Price index for industrial products \\
\hline 923 & Supplies for company kitchens and canteens & StBA F4 R4.2.4 & & & Average German producer price index \\
\hline 931 & Solid fuels & StBA F4 R4.2.4 & GP09-052 & Lignite & Price index for industrial products \\
\hline 932 & Liquid fuels & StBA F4 R4.2.4 & GP09-061 & Petroleum & Price index for industrial products \\
\hline 933 & Gas, e.g., natural gas or liquefied petroleum & StBA F4 R4.2.4 & GP09-062 & Petroleum gas & Price index for industrial products \\
\hline 934 & Electricity & StBA F4 R4.2.4 & GP09-3511 13 & Sales to industrial users & Producer price index for industrial products: \\
\hline \multirow[t]{6}{*}{935} & District heating (steam) and compressed air & StBA F4 R4.2.4 & GP09-353 & $\begin{array}{l}\text { District heating with steam and water } \\
\text { apartment blocks and non-residential } \\
\text { buildings }\end{array}$ & $\begin{array}{l}\text { Producer price index for industrial } \\
\text { products: }\end{array}$ \\
\hline & Costs for hired labour & StBA F4 R4.3 & & & Average price index "costs for labour" \\
\hline & Costs for other industrial and mechanical services & StBA F4 R4.3 & & & Average price index "costs for labour" \\
\hline & Costs for temporary workforces & StBA F4 R4.3 & & & Average price index "costs for labour" \\
\hline & Rents and leases & StBA F4 R4.3 & & & Average German producer price index \\
\hline & Other costs (total) & StBA F4 R4.3 & & & Average German producer price index \\
\hline
\end{tabular}

Appendix D.1. Price and quantity effects of the intermediate input of ES 1610

\begin{tabular}{|c|c|c|c|c|c|c|c|c|}
\hline \multirow[t]{2}{*}{ No. } & \multirow[t]{2}{*}{ Intermediate input of ES 1610} & \multicolumn{3}{|c|}{$\begin{array}{l}\text { Consumption of raw materials and } \\
\text { operating supplies }+ \text { consumption } \\
\text { of commodities in } \mathrm{T} €\end{array}$} & \multicolumn{2}{|c|}{ Relative changes in price } & \multicolumn{2}{|c|}{ Relative changes in quantity } \\
\hline & & \multirow{2}{*}{$\begin{array}{l}2002 \\
(3)\end{array}$} & \multirow{2}{*}{$\begin{array}{l}2006 \\
(4)\end{array}$} & \multirow{2}{*}{$\begin{array}{l}2010 \\
(5)\end{array}$} & \multirow{2}{*}{$\begin{array}{l}2006 \text { compared } \\
\text { to } 2002 \\
(6)\end{array}$} & \multirow{2}{*}{$\begin{array}{l}2010 \text { compared } \\
\text { to } 2006 \\
\overline{(7)}\end{array}$} & \multirow{2}{*}{$\begin{array}{l}2006 \text { compared } \\
\text { to } 2002 \\
(8)\end{array}$} & \multirow{2}{*}{$\begin{array}{l}2010 \text { compared } \\
\text { to } 2006 \\
(9)\end{array}$} \\
\hline$\overline{(1)}$ & $\overline{(2)}$ & & & & & & & \\
\hline I. & \multicolumn{8}{|c|}{ Raw materials and other eternally procured preliminary products and auxiliary supplies (incl. commodities but without fixed assets investments) } \\
\hline 022 & Wood in the rough & $1,174,275$ & $1,706,346$ & $1,842,718$ & 1.067 & 1.264 & 1.361 & 0.854 \\
\hline 161 & Wood, sawn and planed & 539,462 & 830,587 & 546,167 & 1.141 & 1.075 & 1.349 & 0.612 \\
\hline 1621 & Veneer sheets and wood-based panels & 98,103 & 95,238 & 33,136 & 1.095 & 0.988 & 0.886 & 0.352 \\
\hline 1623 & Other builders carpentry and joinery & 28,947 & 143,160 & 23,846 & 0.926 & 1.028 & 5.342 & 0.162 \\
\hline 1629 & $\begin{array}{l}\text { Other products of wood; articles of cork, straw } \\
\text { and plaiting materials }\end{array}$ & 39,370 & 10,796 & 238,945 & 1.125 & 1.091 & 0.244 & 20.295 \\
\hline 25 & $\begin{array}{l}\text { Fabricated metal products, except machinery } \\
\text { and equipment }\end{array}$ & 9751 & 12,658 & 2624 & 1.100 & 1.059 & 1.180 & 0.196 \\
\hline 222 & Plastic products & 39,582 & 29,415 & 5349 & 1.039 & 1.022 & 0.715 & 0.178 \\
\hline 171 & Pulp, paper and paperboard & 2084 & 5276 & 9073 & 0.947 & 1.027 & 2.672 & 1.675 \\
\hline 20 & Chemicals and chemical products & 45,009 & 41,485 & 20,350 & 1.133 & 1.080 & 0.813 & 0.454 \\
\hline 23 & Other non-metallic mineral products & 3005 & 1339 & 10,683 & 1.015 & 1.086 & 0.439 & 7.346 \\
\hline \multirow[t]{2}{*}{900} & Other material and commodity input & 8734 & 14,602 & 13,036 & 1.141 & 1.075 & 1.465 & 0.830 \\
\hline & Non-specified difference to total & 1629 & 1131 & 19,757 & 1.141 & 1.075 & 0.609 & 16.238 \\
\hline II. & \multicolumn{8}{|c|}{ Purchased operating supplies (incl. packaging materials and supplies for company kitchens and canteens, but without fuels) } \\
\hline 921 & Operating materials (without packaging) & 47,970 & 44,841 & 39,280 & 1.137 & 1.040 & 0.822 & 0.843 \\
\hline 922 & Packaging materials, e.g., wood, paper, cardboard & 14,575 & 20,488 & 19,645 & 1.011 & 1.012 & 1.390 & 0.947 \\
\hline 923 & Supplies for company kitchens and canteens & 91 & 62 & 120 & 1.137 & 1.040 & 0.597 & 1.852 \\
\hline III. & \multicolumn{8}{|l|}{ Purchased fuels and energy } \\
\hline 931 & Solid fuels & 667 & 1335 & 10,507 & 1.064 & 1.100 & 1.881 & 7.156 \\
\hline 932 & Liquid fuels & 24,020 & 33,178 & 35,133 & 1.897 & 1.238 & 0.728 & 0.856 \\
\hline 933 & Gas, e.g., natural gas or liquefied petroleum & 3903 & 8854 & 2302 & 1.719 & 0.748 & 1.320 & 0.348 \\
\hline 934 & Electricity & 39,238 & 71,380 & 105,509 & 1.183 & 1.179 & 1.538 & 1.253 \\
\hline \multirow[t]{9}{*}{935} & District heating (steam) and compressed air & 0 & 410 & 13,617 & 1.226 & 1.116 & 0.816 & 29.758 \\
\hline & \multicolumn{8}{|l|}{ Other intermediate input } \\
\hline & Costs for hired labour & 36,365 & 46,382 & 49,111 & 1.035 & 1.068 & 1.232 & 0.991 \\
\hline & Costs for other industrial and mechanical services & 86,126 & 117,509 & 89,612 & 1.000 & 1.000 & 1.364 & 0.763 \\
\hline & Costs for temporary workforces & 5112 & 24,659 & 23,345 & 1.035 & 1.068 & 4.661 & 0.886 \\
\hline & Rents and leases & 52,736 & 81,232 & 78,294 & 1.137 & 1.040 & 1.355 & 0.927 \\
\hline & Other costs (total) & 277,612 & 459,318 & 353,535 & 1.137 & 1.040 & 1.455 & 0.740 \\
\hline & Sum & $2,578,366$ & $3,801,681$ & $3,585,693$ & & & & \\
\hline & Relative change & 1.0 & 1.474 & 1.391 & 1.102 & 1.150 & 1.350 & 0.813 \\
\hline
\end{tabular}




\begin{tabular}{|c|c|c|c|c|c|c|c|c|}
\hline \multirow{3}{*}{$\begin{array}{l}\text { No. } \\
\\
(1)\end{array}$} & \multirow{3}{*}{$\begin{array}{l}\text { Intermediate input of ES } 1621 \\
(2)\end{array}$} & \multicolumn{3}{|c|}{$\begin{array}{l}\text { Consumption of raw materials and } \\
\text { operating supplies }+ \text { consumption of } \\
\text { commodities in } \mathrm{T} €\end{array}$} & \multicolumn{2}{|c|}{ Relative changes in price } & \multicolumn{2}{|c|}{ Relative changes in quantity } \\
\hline & & \multirow{2}{*}{2002} & \multirow{2}{*}{2006} & \multirow{2}{*}{2010} & \multirow{2}{*}{$\begin{array}{l}2006 \text { compared } \\
\text { to } 2002 \\
(6)\end{array}$} & \multirow{2}{*}{$\begin{array}{l}2010 \text { compared } \\
\text { to } 2006 \\
(7)\end{array}$} & \multirow{2}{*}{$\begin{array}{l}2006 \text { compared } \\
\text { to } 2002 \\
(8)\end{array}$} & \multirow{2}{*}{$\begin{array}{l}2010 \text { comparec } \\
\text { to } 2006 \\
(9)\end{array}$} \\
\hline & & & & & & & & \\
\hline I. & \multicolumn{8}{|c|}{ Raw materials and other eternally procured preliminary products and auxiliary supplies (incl. commodities but without fixed assets investments) } \\
\hline 022 & Wood in the rough & 326,920 & 365,584 & 682,112 & 1.067 & 1.264 & 1.048 & 1.476 \\
\hline 161 & Wood, sawn and planed & 194,534 & 402,183 & 181,467 & 1.141 & 1.075 & 1.812 & 0.420 \\
\hline 1621 & Veneer sheets and wood-based panels & 547,154 & 639,600 & $1,040,550$ & 1.095 & 0.988 & 1.067 & 1.646 \\
\hline 1623 & Other builders carpentry and joinery & 91,872 & 6807 & 0 & 0.926 & 1.028 & 0.080 & 0.000 \\
\hline 1629 & $\begin{array}{l}\text { Other products of wood; articles of cork, straw } \\
\text { and plaiting materials }\end{array}$ & 6608 & 28,678 & 13,273 & 1.125 & 1.091 & 3.857 & 0.424 \\
\hline 25 & $\begin{array}{l}\text { Fabricated metal products, except machinery } \\
\text { and equipment }\end{array}$ & 0 & 6807 & 3749 & 1.036 & 1.096 & 0.965 & 0.502 \\
\hline 222 & Plastic products & 14,037 & 3970 & 8313 & 1.100 & 1.059 & 0.257 & 1.977 \\
\hline 171 & Pulp, paper and paperboard & 99,168 & 82,047 & 100,902 & 1.039 & 1.022 & 0.796 & 1.203 \\
\hline 20 & Chemicals and chemical products & 288,386 & 439,574 & 289,643 & 0.947 & 1.027 & 1.609 & 0.642 \\
\hline 23 & Other non-metallic mineral products & 501,439 & 666,509 & 479,736 & 1.133 & 1.080 & 1.173 & 0.667 \\
\hline 900 & Other material and commodity input & 32,743 & 33,262 & 21,577 & 1.141 & 1.075 & 0.890 & 0.603 \\
\hline & Non-specified difference to total & 657 & 7779 & 34,201 & 1.141 & 1.075 & 10.371 & 4.089 \\
\hline II. & \multicolumn{8}{|c|}{ Purchased operating supplies (incl. packaging materials and supplies for company kitchens and canteens, but without fuels) } \\
\hline 921 & Operating materials (without packaging) & 98,125 & 116,409 & 0 & 1.137 & 1.040 & 1.043 & 0.000 \\
\hline 922 & Packaging materials, e.g., wood, paper, cardboard & 29,029 & 35,648 & 38,173 & 1.011 & 1.012 & 1.214 & 1.058 \\
\hline 923 & Supplies for company kitchens and canteens & 268 & 7 & 0 & 1.137 & 1.040 & 0.023 & 0.000 \\
\hline III. & \multicolumn{8}{|l|}{ Purchased fuels and energy } \\
\hline 931 & Solid fuels & 0 & 7422 & 18,313 & 1.064 & 1.100 & 0.939 & 2.243 \\
\hline 932 & Liquid fuels & 78,375 & 24,785 & 17,620 & 1.897 & 1.238 & 0.167 & 0.574 \\
\hline 933 & Gas, e.g., natural gas or liquefied petroleum & 0 & 79,461 & 57,347 & 1.719 & 0.748 & 0.582 & 0.965 \\
\hline 934 & Electricity & 107,064 & 178,826 & 197,604 & 1.183 & 1.179 & 1.412 & 0.937 \\
\hline \multirow[t]{9}{*}{935} & District heating (steam) and compressed air & 9253 & 25,331 & 16,515 & 1.226 & 1.116 & 2.233 & 0.584 \\
\hline & \multicolumn{8}{|l|}{ Other intermediate input } \\
\hline & Costs for hired labour & 23,632 & 31,663 & 41,759 & 1.035 & 1.068 & 1.295 & 1.234 \\
\hline & $\begin{array}{l}\text { Costs for other industrial and mechanical } \\
\text { services }\end{array}$ & 132,562 & 146,764 & 118,941 & 1.000 & 1.000 & 1.107 & 0.810 \\
\hline & Costs for temporary workforces & 18,829 & 26,973 & 26,458 & 1.035 & 1.068 & 1.384 & 0.918 \\
\hline & Rents and leases & 85,903 & 97,533 & 97,058 & 1.137 & 1.040 & 0.998 & 0.957 \\
\hline & Other costs (total) & 493,419 & 689,570 & 572,827 & 1.137 & 1.040 & 1.229 & 0.799 \\
\hline & Sum & $3,179,979$ & $4,143,193$ & $4,058,140$ & & & & \\
\hline & Relative change & 1.0 & 1.303 & 1.276 & 1.108 & 1.061 & 1.164 & 0.921 \\
\hline
\end{tabular}

\section{Appendix E. Price and quantity changes of ES 1610 and ES 1621}

\begin{tabular}{|c|c|c|c|c|}
\hline \multirow[t]{2}{*}{ ES 1610} & \multicolumn{2}{|c|}{ Base year 2002} & \multicolumn{2}{|c|}{ Base year 2006} \\
\hline & 2002 & 2006 & 2006 & 2010 \\
\hline Gross production value (GPV) in $\mathrm{T} €$ & $3,397,623$ & $4,861,283$ & $4,861,283$ & $4,443,261$ \\
\hline Relative change of GPV & 1.0 & 1.431 & 1.0 & 0.914 \\
\hline Producer price index ES 1610 & 81.5 & 93 & 93 & 100 \\
\hline Relative change of producer prices & 1.0 & 1.141 & 1.0 & 1.075 \\
\hline Output quantity & $3,397,623$ & $4,260,157$ & $4,861,283$ & $4,132,233$ \\
\hline Relative change of output quantity & 1.0 & 1.254 & 1.0 & 0.850 \\
\hline Intermediate input in $\mathrm{T} €$ & $2,578,366$ & $3,801,681$ & $3,801,681$ & $3,585,693$ \\
\hline Weighted average of changes of int. input prices & 1.0 & 1.102 & 1.0 & 1.150 \\
\hline Intermediate input quantity & $2,578,366$ & $3,450,438$ & $3,801,681$ & $3,118,015$ \\
\hline Weighted average of changes of int. input quantity & 1.0 & 1.350 & 1.0 & 0.813 \\
\hline Share of intermediate input of GPV & 0.76 & 0.78 & 0.78 & 0.81 \\
\hline Gross value added (GVA) in T€ & 819,257 & $1,059,602$ & $1,059,602$ & 857,568 \\
\hline Relative changes of GVA & 1.0 & 1.293 & 1.0 & 0.809 \\
\hline Differences to base year of GVA in T€ & 0.0 & 240,345 & 0.0 & $-202,034$ \\
\hline
\end{tabular}

\section{References}

Ang, B.W., 1995. Decomposition methodology in industrial energy demand analysis. Energy 20 (11), 1081-1095.

Ang, B.W., Zhang, F.Q., Ki-Hong, C., 1998. Factorizing changes in energy and environmental indicators through decomposition. Energy 23 (6), 489-495.

Bundesministerium für Verbraucherschutz, Ernährung und Landwirtschaft (BMVEL) (Ed.), 2004. Verstärkte Holznutzung: Zugunsten von Klima, Lebensqualität, Innovation und Arbeitsplätzen (Charta für Holz). The
Bundesministerium für Verbraucherschutz, Ernährung und Landwirtschaft (BMVEL), Berlin.

Dieter, M., 2005. Holzbilanzen 2002, 2003 und 2004 für die Bundesrepublik Deutschland. Institut für Ökonomie der Bundesforschungsanstalt für Forst- und Holzwirtschaft, Hamburg (Arbeitsbericht 2005/3, http://www.bfafh.de/bibl/ pdf/iii_05_03.pdf).

Dieter, M., 2006. Holzbilanzen 2004 und 2005 für die Bundesrepublik Deutschland. Arbeitsbericht des Instituts für Ökonomie 2006/2. Johann-Heinrich-von-ThünenInstitut, Hamburg. 
Dieter, M., 2007. Holzbilanzen 2005 und 2006 für die Bundesrepublik Deutschland. Arbeitsbericht des Instituts für Ökonomie 2007/2. Johann-Heinrich-von-ThünenInstitut, Hamburg.

Dieter, M., 2008. Analyse der Wertschöpfung durch Holznutzung aus gesamtwirtschaftlicher Perspektive. Allgemeine Forst- und Jagdzeitung. (German Journal of Forest Research) 179. Frankfurt am Main: J. D. Sauerländer's Verlag, pp. 202-207 (Jahrgang, Heft 10/11, S).

Dieter, M., 2010. Produktion, Weiterverarbeitung und Handel von Holz aus gesamtwirtschaftlicher Perspektive - eine quantitative Analyse der Forst- und Holzwirtschaft auf Grundlage sektoraler Statistiken. Habilitationsschrift zur Erlangung der venia legendi im Fachgebiet Forstökonomie. Georg-August-Universität Göttingen, Fakultät für Forstwissenschaften und Waldökologie.

Dieter, M., Englert, H., 2007. Competitiveness in the global forest industry sector: an empirical study with special emphasis on Germany. Eur. J. For. Res. 126, 401-412.

Dieter, M., Englert, H., 2009. Zur Wettbewerbsfähigkeit der europäischen Holzwirtschaft eine Constant-Market-Share-Analyse. Schweiz. Z. Forstwes. 160 (12), 375-383.

Ermisch, N., Seintsch, B., Dieter, M., 2013. Holzartengruppe Fichte 2003 bis 2011 konstant mit höchstem Erlösbeitrag. AFZ-Der Wald 23/2013, 6-9.

Frenkel, M., John, K.D., 1999. Volkswirtschaftliche Gesamtrechnung. Franz Vahlen, München.

Ivanov, S., Webster, C., 2010. Decomposition of economic growth in Bulgaria by industry. J. Econ. Stud. 37 (2), 219-227.

Klein, D., Kies, U., Schulte, A., 2009. Regional employment trends of wood-based industries in Germany's forest cluster: a comparative shift-share analysis in postreunification development. Eur. J. For. Res. 128, 205-219.

Mantau, U., 2009. Holzrohstoffbilanz Deutschland: Szenarien des Holzaufkommens und der Holzverwendung bis 2012. Landbauforschung vTI agriculture and forestry research - Sonderheft, Band 327 pp. 27-36 (Braunschweig, S).

Mantau, U., 2012. Holzrohstoffbilanz Deutschland. Entwicklungen und Szenarien des Holzaufkommens und der Holzverwendung von 1987 bis 2015. Universität Hamburg, Zentrum Holzwirtschaft, Arbeitsbereich Ökonomie der Holz- und Forstwirtschaft, Hamburg.

Milana, C., 1988. Constant-market-share: an analysis and index number theory. Eur. J. Polit. Econ. 4, 453-478.

O'Donnell, C.J., 2012. An aggregate quantity framework for measuring and decomposing productivity change. J. Prod. Anal. 38, 255-272.

Ochs, T., Duschl, C., Seintsch, B., 2007. Struktur und Rohstoffbedarf der Holzwirtschaft: Teil I der Studie "Regionalisierte Struktur- und Marktanalyse der 1. Verarbeitungsstufe der Holzwirtschaft". Holz-Zentralblatt 133 (10), 269-271.

Sakamoto, H., Fan, J., 2009. Regional income disparity in China using value-added data: decomposition and distribution dynamics. Rev. Urban Reg. Dev. Stud. 25 (1), 16-33.

Seintsch, B., 2010. Holzbilanzen 2006 bis 2009 für die Bundesrepublik Deutschland. vTI, Arbeitsbericht des Thünen Instituts für Ökonomie der Forst- und Holzwirtschaft 2010/03. Johann Heinrich von Thünen Institut, Hamburg.
Seintsch, B., 2011a. Holzbilanzen 2009 und 2010 für die Bundesrepublik Deutschland. Arbeitsbericht des Instituts für Ökonomie der Forst- und Holzwirtschaft 2011/04. Johann-Heinrich-von-Thünen-Institut, Hamburg.

Seintsch, B., 2011b. Stellung der Holzrohstoffe in der Kostenstruktur des Holz- und Papiergewerbes in Deutschland. Arbeitsbericht des Instituts für Ökonomie der Forst- und Holzwirtschaft 03/2011. Johann Heinrich von Thünen Institut, Hamburg.

Seintsch, B., 2013. Cluster Forst und Holz nach neuer Wirtschaftszweigklassifikation. Tabellen für das Bundesgebiet und die Länder 2000 bis 2011. Thünen Working Paper 5. Thünen-Institut für Forstökonomie, Hamburg/Germany.

Seintsch, B., Weimar, H., 2013. Holzbilanzen 2010 bis 2012 für die Bundesrepublik Deutschland. Thünen Working Paper 9. Johann Heinrich von Thünen-Institut, Hamburg.

Sinn, H.-W., 2005. Basar-Ökonomie Deutschland: Exportweltmeister oder Schlusslicht? Ifo Schnelldienst 6/2005, ifo Institut für Wirtschaftsforschung e.V., 58. Jg., 12.-13. KW, 31. März 2005

Statistisches Bundesamt (StBA): Produktionserhebungen (F4 R3.1) Wiesbaden (annual appearance). Statistische Bundesamt.

Statistisches Bundesamt (StBA): Beschäftigung und Umsatz der Betriebe des Verarbeitenden Gewerbes sowie des Bergbaus und der Gewinnung von Steinen und Erden (F4 R 4.1.1), Wiesbaden (annual appearance). Statistische Bundesamt.

Statistisches Bundesamt (StBA): Material- und Wareneingangserhebung im Verarbeitenden Gewerbe sowie im Bergbau und in der Gewinnung von Steinen und Erden (F4 R4.2.4) Wiesbaden (annual appearance). Statistische Bundesamt

Statistisches Bundesamt (StBA): Kostenstruktur der Unternehmen des Verarbeitenden Gewerbes sowie des Bergbaus und der Gewinnung von Steinen und Erden (F4 R4.3) Wiesbaden (annual appearance). Statistische Bundesamt

Statistisches Bundesamt (StBA): Außenhandel nach Waren und Ländern (F7 R2), Wiesbaden (annual appearance). Statistische Bundesamt

Statistisches Bundesamt (StBA): Arbeitskosten im Produzierenden Gewerbe und im Dienstleistungsbereich (F16 R 1 ) (quarterly appearance). Statistische Bundesamt

Statistisches Bundesamt (StBA): Index der Erzeugerpreise der Produkte des Holzeinschlags aus den Staatsforsten (F17 R1), Wiesbaden (annual appearance). Statistische Bundesamt

Statistisches Bundesamt (StBA): Index der Erzeugerpreise gewerblicher Produkte Deutschland (F17 R2) Wiesbaden (annual appearance). Statistische Bundesamt

Sun, J.W., Ang, B.W., 2000. Some properties of an exact energy decomposition model. Energy 25, 1177-1188.

Weimar, H., 2011. Der Holzfluss in der Bundesrepublik Deutschland 2009: Methode und Ergebnis der Modellierung des Stoffflusses von Holz. vTI, Arbeitsbericht des Instituts für Ökonomie der Forst- und Holzwirtschaft 2011/06. Johann Heinrich von Thünen Institut, Hamburg. 


\section{Wissenschaftlicher Werdegang}

Lydia Christina Antonia Rosenkranz, geb. Gallon

geboren 12.03.1980 in Bonn, deutsch, verheiratet, 1 Kind

04/2001 - 01/2006 Studium der Forstwissenschaften, Albert-Ludwigs-Universität Freiburg Abschluss: Diplom-Forstwirtin

09/2002 bis 01/2003: Teilnahme am ERASMUS-Programm der EU "School of Agricultur and Forest Sciences", Bangor, Wales

07/2006 - 06/2008 Referendarin im höheren Forstdienst, Landesforsten Rheinland - Pfalz Abschluss: Forstassessorin

11/2008 bis 01/2010 Wissenschaftliche Mitarbeiterin am Institut für Forst- und Umweltpolitik, Arbeitsbereich Forstpolitik, Albert-Ludwigs-Universität Freiburg

01/2010 - 09/2013 Wissenschaftliche Mitarbeiterin am Johann Heinrich von Thünen-Institut, Institut für Ökonomie der Forst- und Holzwirtschaft, Hamburg

07/2013 - 03/2016 Promotionsstudium an der Fakultät für Forstwissenschaften und Waldökologie der Georg-August-Universität Göttingen

seit $10 / 2013$ Wissenschaftliche Mitarbeiterin am Thünen-Institut für Internationale Waldwirtschaft und Forstökonomie, Hamburg 


\section{Danksagung}

Mein Dank gilt meinem Erstgutachter, Prof. Dr. Matthias Dieter, für die Möglichkeit meine Dissertation an seinem Institut, im Rahmen spannender wissenschaftlicher Projekte anzufertigen sowie für seine erstklassige fachliche Unterstützung, die anregenden Diskussionen und die konstruktive Kritik meiner Arbeit.

Bei Prof. Dr. Bernhard Möhring bedanke ich mich für die wertvolle Unterstützung im Laufe der letzten Jahre, insbesondere im Projekt FFH-Impact, und für sein Einverständnis, Zweitgutachter dieser Arbeit zu sein.

Ein besonderer Dank gilt Dr. Björn Seintsch für die gute und nette Zusammenarbeit, die intensiven Gespräche und vielfältigen fachlichen Anregungen. Ohne seine großartige Unterstützung wäre diese Arbeit nicht möglich gewesen.

Hermann Englert danke ich für die technische und fachliche Unterstützung insbesondere bei den Modellarbeiten und der Dekompositionsanalyse.

Weiterhin danke ich Dr. Bernd Wippel für die nette und wertvolle Zusammenarbeit im Projekt FFHImpact, dessen Ergebnisse einen wesentlichen Teil dieser Arbeit ausmachen.

Meinen Kollegen am Thünen-Institut für Internationale Waldwirtschaft und Forstökonomie danke ich für ihre große Hilfsbereitschaft und die immer nette Arbeitsatmosphäre.

Nicht zuletzt danke ich meiner Familie und meinem Mann, Florian Rosenkranz, für ihre Liebe, ihre Unterstützung entlang meines Lebenswegs und ihr Vertrauen in mich. 


\section{Eidesstattliche Erklärung}

Hiermit erkläre ich an Eides statt, dass ich die beigefügte Dissertation selbständig und ohne unerlaubte Hilfe angefertigt habe. Alle wörtlich oder inhaltlich übernommenen Stellen habe ich als solche gekennzeichnet.

Ich versichere, dass ich die beigefügte Dissertation nur in diesem und keinem anderen Promotionsverfahren eingereicht habe.

Ich versichere außerdem, dass ich mich vor diesem Promotionsverfahren keiner weiteren Promotionsprüfung unterzogen oder mich zu einer solchen Prüfung angemeldet habe.

Hamburg, den 14.11.2015

Lydia Rosenkranz 